\title{
Design, Fabrication and Calibration of Compliant, Multi-Axis, Fiber-Optic Force/Torque Sensors for Biomechanical Measurements
}

BY

\begin{abstract}
Osama Al-Mai
A thesis submitted to the Faculty of Graduate and Postdoctoral AfFAiRs in PARTial FUlfillment of THE REQUIREMENTS FOR THE DEGREE OF
\end{abstract}

Doctor of Philosophy in Mechanical Engineering

OTTAWA-CARLETON INSTITUTE FOR

Mechanical and Aerospace Engineering

Department of Mechanical and Aerospace Engineering

CARLETON UNIVERSITY

Ottawa, Ontario, Canada

(C)Osama Al-Mai

November 5, 2019 
THE Undersigned HEREBY RECOMMENDS TO THE

Faculty of Graduate and Postdoctoral Affairs

ACCEPTANCE OF THE THESIS

Design, Fabrication and Calibration of Compliant, MultiAxis, Fiber-Optic Force/Torque Sensors for Biomechanical

Measurements

SUBmitted By Osama Al-Mai

IN PARTIAL FULFILMENT OF THE REQUIREMENTS FOR THE DEGREE OF

Doctor of Philosophy in Mechanical Engineering

Carleton University

November 5, 2019 


\section{Abstract}

This thesis presents the design, fabrication and characterization of various prototypes of multi-axis, compliant force and torque sensors based on fiber-optic sensing technology, the novel calibration methodologies and the experimental results. A compliant 3-axis, intensity modulated-based, fiber-optic force sensor that simultaneously measures normal and shear forces was designed, prototyped and successfully calibrated. A nonlinear Hammerstein-Weiner model (NLHW) was able to characterize the linear and nonlinear behaviour of this prototype. The optimized results have shown a reduction of over $40 \%$ in the Root Mean Square Errors (RMSE) in comparison with the linear estimation models. For biomechanical applications such as ground reaction force and gait measurements, the sensor must be able to measure the complete degree of freedom of any force or torque applied at a certain point. Therefore, a wearable compliant 6-axis force and torque sensor was developed and prototyped. It combines two different force sensing technologies: the 3-axis fiber-optic based force sensor and a pressure sensor matrix. The sensor was capable of accurately measuring the full ground reaction force and moment in real-time with minimal gait disturbance.

To enhance the durability, avoid the necessary multi-stage conditioning circuits and their resulting extra electronic components, a 6-axis force and torque sensor that is fully optical has been developed and characterized. The sensor is cost-effective, lightweight and flexible with a large force and torque measurement range suitable for biomechanics and rehabilitation systems. A novel calibration methodology which splits the calibration procedure into two estimation models that work simultaneously as a single calibration system named Least Squares Decision Trees (LSDT). Using LSDT, the estimation speed increased by $55.17 \%$ and the RMSE reduced to $0.53 \%$. 
To improve sensor portability, further reduce size and eliminate electromagnetic interference effects as well as enhance sensor biocompatibility, a non-conductive, electrically passive, fiber Bragg grating (FBG) based normal and shear force sensing elements were designed, fabricated and calibrated. The sensing elements are small size, lightweight and compliant. The results achieved from the proposed calibration method have revealed an improvement from an R-squared value of $93 \%$ to $100 \%$ when compared to a data obtained using a linear least squares method. 


\section{Acknowledgments}

In 2015 when I met Professor Mojtaba Ahmadi, he introduced me to the Advanced Biomechatronics Locomotion (ABL) laboratory and he gave me the opportunity to work on the development of compliant force and torque sensors as a part of the running BalanceAid project. I would like to thank him and my co-supervisor Professor Jacques Albert for their valuable guidance, consistent encouragements and academic support. With their powerful supervision my Ph.D studies has been a remarkable journey of several stages, full of challenges, perseverance and hard work. I would also like to thank the department of Mechanical and Aerospace Engineering for letting me use the Bionix Servo-hydraulic Test System, 3D printer and the workshop to carry out experiments, develop force and torque sensors and all research work.

I would particularly like to thank the technologists and staff in the department, Albane Laronche, David Raude, Stephan Biljan, and Steve Truttman for their assistance in all things performed throughout my PhD studies. A special thanks to all my family members for all of sacrifices they have made on my behalf. Their prayer for me is what sustained me in the course of this research. Last but not least, I would like to thank the present and the past members of the ABL lab for their valuable technical discussions that are being held daily in the lab and every individual who was a source of encouragement and support and helped me to accomplish my dream goals. 


\section{Preface}

As defined by section 12.4 of 2019-2020 Graduate Calendar, the current thesis is an integrated Ph.D thesis. It includes the following articles which are either published (paper 1, 2, 3 and 4) or are submitted ( paper 5 and 6):

1. O. Al-Mai, M. Ahmadi, and J. Albert, "A Compliant 3-Axis Fiber-Optic Force Sensor for Biomechanical Measurement," IEEE Sensors Journal, vol. 17, no. 20, pp. 6549-6557, 2017 [1].

2. O. Al-Mai, M. Ahmadi, and J. Albert, " Design, Development and Calibration of a Lightweight, Compliant Six-Axis Optical Force/Torque Sensor, " IEEE Sensors Journal, vol. 18, no. 17, pp. 7005-7014, 2018 [2].

3. S. Eng, O. Al-Mai, and M. Ahmadi, "A 6 DoF, Wearable, Compliant Shoe Sensor for Total Ground Reaction Measurement," IEEE Transactions on Instrumentation and Measurement, vol. 67, no. 11, pp. 2714-2722, 2018 [3].

4. O. Al-Mai, M. Ahmadi, and J. Albert, "Fiber-Optic Based, Force and Torque Compliant Sensing Calibration Strategies , "International Conference of Control, Dynamic Systems, and Robotics , 2019. [4]

5. O. Al-Mai, M. Ahmadi, and J. Albert, "Development and Characterization of A Compliant FBG-Based, Shear and Normal Force Sensing Elements for Biomechanical Applications," submitted to IEEE Sensors Journal, 2019. 
6. O. Al-Mai, and M. Ahmadi, "Novel Calibration Methodologies for Compliant Multi-Axis Fiber-Optic Based Force/Torque Sensors," submitted to IEEE Transactions on Instrumentation and Measurement, 2019.

The permission from the publisher (IEEE) has been included for each journal paper at the end of this thesis. The format of these journal papers has been transformed to be more coherent to the format of the current thesis. Also, the images and plots have been re-centred and rescaled. Sections of additional work have also been added to introduce materials included in the Appendix. The references have been created in a single list that can be found at the end of this thesis. 


\section{Contents}

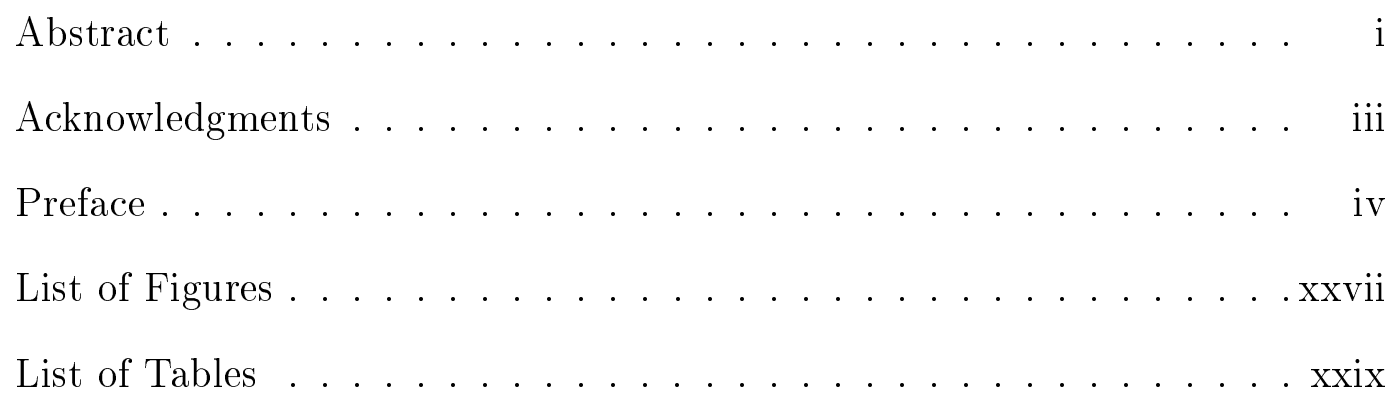

1 Introduction 1

1.1 Motivation . . . . . . . . . . . . . . . . . 1

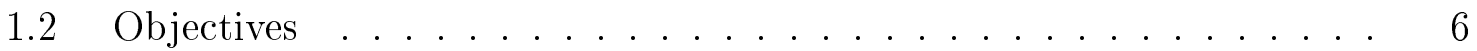

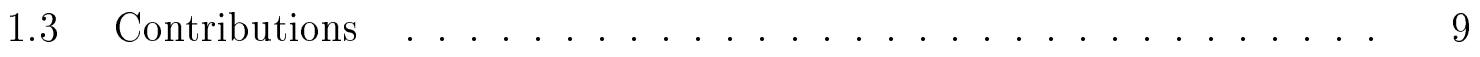

1.4 Outline ............................. 12

2 Background and Literature Review $\quad 14$

2.1 Measurement Proficiency and Advantages of Fiber-Optic Sensors . . . . 14

2.2 Strengths and Limitations of Current Biomedical and Biomechanical Measurement Systems . . . . . . . . . . . . . . 16

2.3 Intensity Modulated-Based Fiber-Optic Sensors _ . . . . . . . . . 18

2.3.1 Microbending-Based Fiber-Optic Sensors . . . . . . . . . . 19

2.3.2 Reflection Based Fiber-Optic Sensors . . . . . . . . . . . 23 
2.3.2.1 Reflecting-Based Minimally-Invasive Surgical Force Sensors with Catheter-Tip . . . . . . . . 25

2.3.2.2 Hand-grip Force Sensor with MRI Compatibility Based on Light Reflection . . . . . . . . . . . 26

2.3.3 Shutter-Based Intensity Modulation Fiber Optic Sensors . . 28

2.4 Wavelength-Modulated Fiber-Optic Sensors $\ldots \ldots \ldots \ldots$

2.4 .1 FBG Fabrication $\ldots \ldots \ldots \ldots \ldots \ldots \ldots$

2.4.2 FBG Sensing Principles . . . . . . . . . . . . . . 32

2.4.3 FBG-Based Sensors in Biomechanical and Gait

Measurements . . . . . . . . . . . . . 37

\section{A Compliant 3-Axis Fiber-Optic Force Sensor for Biomechanical} $\begin{array}{ll}\text { Measurement } & 44\end{array}$

3.1 Introduction . . . . . . . . . . . . . . . . . . 45

3.2 Sensor Design and Fabrication . . . . . . . . . . . . . . . . 49

3.3 Sensing Mechanism and Working Principles . . . . . . . . . . 52

3.4 Experimental Results and Calibration . . . . . . . . . . . . 54

3.4.1 The Effect of Cross-talk on Sensor Performance . . . . . . . 57

3.4 .2 Calibration Methodology . . . . . . . . . . 61

3.4 .3 Nonlinear Hammerstein-Wiener Model . . . . . . . . . . . 65

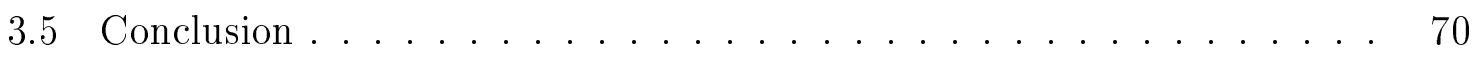

4 A 6-DoF, Wearable, Compliant Shoe Sensor for Total Ground $\begin{array}{ll}\text { Reaction Measurements } & 72\end{array}$

4.1 Introduction $\ldots \ldots \ldots \ldots \ldots \ldots \ldots \ldots \ldots \ldots$

4.2 Sensor Design . . . . . . . . . . . . . . . . 76 
4.3 Fiber-Optic Shear Force Sensor ～. . . . . . . . . . . . . 77

4.4 FlexiForce Sensors _ . . . . . . . . . . . . . . . . 80

4.5 Experimental Results . . . . . . . . . . . . . . . . . . . 81

4.5.1 Floor-mounted experiments . . . . . . . . . . . . 82

4.5.1.1 Least squares regression . . . . . . . . . . . . 82

4.5.1.2 Neural network regression . . . . . . . . . . . 84

4.5.2 Shoe-mounted experiments . . . . . . . . . . . . . 85

4.5.2.1 Least squares regression . . . . . . . . . . . . 92

4.5.2.2 Neural network regression . . . . . . . . . . . . 92

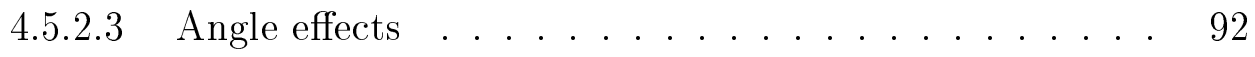

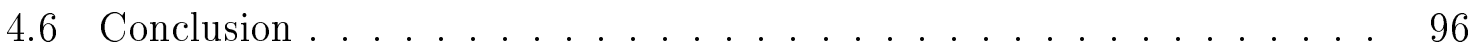

5 Design, Development and Calibration of a Lightweight, Compliant 6-Axis Optical Force/Torque Sensor $\quad 98$

5.1 Introduction . . . . . . . . . . . . . . . . . . . . . 99

5.2 Sensor Architecture . . . . . . . . . . . . . . . . . . 103

$5.2 .1 \quad$ Sensor Design and Fabrication . . . . . . . . . . . . 103

5.2 .2 Principles of sensing $\ldots \ldots \ldots \ldots \ldots$

5.3 Sensor Characterization $\ldots \ldots \ldots \ldots$

$5.3 .1 \quad$ Experimental setup $\ldots \ldots \ldots \ldots \ldots \ldots$

5.3.2 Linear Estimation via Least Squares Method (LSM) _. . . . 112

5.3.3 Nonlinear Regression Using Decision Trees . . . . . . . . . . 114

5.3.4 Least Squares Decision Trees LSDT Approach . . . . . . . 117

5.4 Conclusion . . . . . . . . . . . . . . . . . . . . 123 
6 Development and Characterization of Compliant FBG-Based, Shear and Normal Force Sensing Elements for Biomechanical Applications 126

6.1 Introduction . . . . . . . . . . . . . . . . . . . . 128

6.2 FBG-Based Normal and Shear Force Sensing Ele- ments: Design and

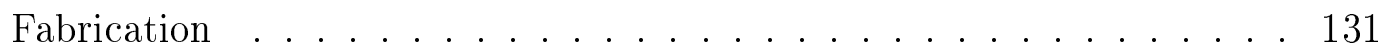

6.3 Sensing Mechanism and Operating Principles . . . . . . . . . . 135

6.4 Experimental Setup . . . . . . . . . . . . . . . . . . . . 139

6.5 Sensor Calibration Methodology: Results and

Discussion . . . . . . . . . . . . . . . . 140

6.5.1 Nonlinear System Identifcation using Nonlinear Autoreg- ressive Exogenous NARX Model . . . . . . . . . . . . . . . . 142

6.5.2 Linear Regression with A Nonlinear Autoregressive Exogenous (LR-NARX) Calibration Procedure . . . . . . 146

6.5.3 Spline Regression for Sensor characterization . . . . . . . . 153

6.6 Conclusion . . . . . . . . . . . . . . . . . . . 156

7 Novel Calibration Methodologies for Compliant Multi-Axis $\begin{array}{ll}\text { Force/Torque Sensors } & 158\end{array}$

7.1 Introduction . . . . . . . . . . . . . . . . . . . . . 159

7.2 knowledge-Based (Parametric) Calibration Meth- odologies . . . . . . 162

7.2.1 Data-Driven Dynamic Modelling . . . . . . . . . . 162

7.2 .2 Hysteresis Compensation . . . . . . . . . . . . 165

7.2 .3 Static Calibration . . . . . . . . . . . 165

7.3 Non-Parametric Machine Learning Based Calibr- ation . . . . . . . 171

7.4 Two-Model Hybrid Calibration Methodology . . . . . . . . . . . . 175

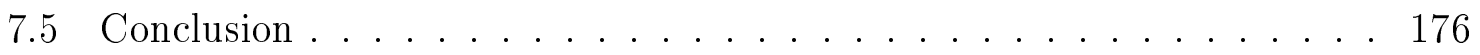


8.1 Conclusions . . . . . . . . . . . . . . . . . . . . . 179

8.1.1 Design and Implementation of A Compliant 3-Axis Fiber-Optic Based Force Sensor . . . . . . . . . . . . 179

8.1.2 Development of 6-DoF Sensor for Total Ground Reaction Force Based on Two Sensing Technologies _ . . . . . . . 180

8.1.3 Design, Development and Calibration of a Lightweight, Compliant Six-Axis Optical Force/Torque Sensor . . . . . . . 181

8.1.4 Development of FBG-Based Normal and Shear Force Sensing Elements . . . . . . . . . . . . . . 182

8.1.5 Novel Calibration Methodologies for Compliant Multi- Axis Force/Torque Sensors . . . . . . . . . . . . . . . 182

8.2 Future Work . . . . . . . . . . . . . . . . . . . . . . 183

8.2.1 Sensor Modifications and Improvements . . . . . . . . . . 183

8.2.2 Development of FBG-Based, 6-Dof insole Sensor for Total Ground Reaction Force and Moment . . . . . . . . . . . 184

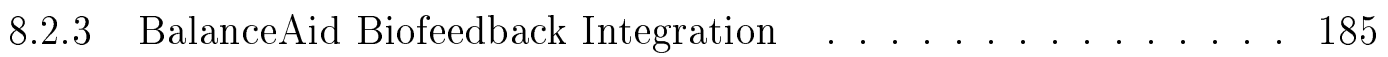

8.2.4 Assistive Device Feedback Integration . . . . . . . . . 186

$\begin{array}{ll}\text { References } & 187\end{array}$

$\begin{array}{ll}\text { Appendices } & 235\end{array}$

A Experimental Results of the light intensity Modulation-Based, 3-Axis Force Sensor

A.1 Experimental Validation Through An Actual Gait Measurement Scenario236

A.2 Specification of the compliant Force Sensor _ . . . . . . . . 247 
B Experimental Results of the intensity-based, 6-Axis F/T Sensor 250

B.1 Experimental Validation Through An Actual Gait Measurement Scenario 250

B.2 Frequency Response Function . . . . . . . . . . . . . 258

C FBG-Based Force Sensing Elements: Experimental Results 


\section{List of Figures}

2.1 Fiber-optic sensors classification: (a) Intrinsic sensors (b) Extrinsic sensors $[5] . \ldots \ldots \ldots \ldots \ldots$

2.2 Radiative losses as a fiber undergoes bends [6]. . . . . . . . . . . 19

2.3 plantar and shear pressure sensor prototype [7]. . . . . . . . . . . . . 21

2.4 plantar and shear pressure sensor prototype [8]. . . . . . . . . . . 22

2.5 Pressure insole developed based on bend loss sensing technique [9]. . . 23

2.6 Schematic of an optical fibers located in the front of a movable reflecting surface. The intensity of the reflected light increases when the distance between the tip of fibers and the reflector decreases. . . . . . . . . 24

2.7 Intensity-modulated reflected light force sensor catheters (a) having triaxial sensing with three optical fibers and (b) having uniaxial sensing with six optical fibers $[10,11] . \ldots \ldots \ldots . \ldots . \ldots 25$

2.8 Hand-Grip Fiber-Optic Force Sensors. (a) metal elastic body and (b) the 3D printed body structure $[12,13] . \ldots \ldots \ldots 27$

2.9 Fiber-optic displacement sensors. (a) Measure the displacement directly using two opposite fibers. (b) a moveable shutter is used to modulate the intensity of light to detect the displacement change. . . . . . . . 28 
2.10 Optoelectronic sensing element without the use of optical fibers to measure the vertical ground reaction force [14]. . . . . . . . . . 29

2.11 Model FBG theory . . . . . . . . . . . . . . . 31

2.12 Light propagation through the FBG sensing element. A narrowband of the transmitted light with specific wavelength reflects back due to the Bragg gratings. ...................... 33

2.13 FBG sensing element and the reflected wavelength variation under uniaxial strain. . . . . . . . . . . . . . . . 34

2.14 Strain and temperature sensitivities of an FBG-based sensor [15]. . . . 36

2.15 FBG-based sensor plantar normal force measurements. The FBG is integrated inside a 3D printed cylindrical bar through a slot made at the center of each sensing element [16]. . . . . . . . . . . . 38

2.16 A flexible insole force sensor composed of six FBG sensing elements for distributive plantar normal pressure measurements [17] . . . . . . . . 39

2.17 A rigid in-shoe shear force sensor affixed between to plates [18]. . . . . 40

2.18 FBG-based sensing element for shear force measurements [19, 20]. . . 40

2.19 FBG-based sensing element for normal and shear forces measurements[20]. 41

2.20 A flexible FBG-based force sensor for normal force and one component of shear force detection [21]. . . . . . . . . . . . . . . . . 42

2.21 Instrumented insole sensor with six FBG sensing elements for vertical ground reaction force detection [22]. . . . . . . . . . . . . 43 
3.1 The fiber-optic sensing principles for the simultaneous measurement of normal and shear forces in the $\mathrm{z}, \mathrm{x}$ and $\mathrm{y}$ directions. (1), (7). The transmitting and receiving fibers of y-axis force sensor. (2). Reflective surfaces. (3), (4). Z-Axis transmitting and receiving fibers. (5), (6). The transmitting and receiving fibers of the $\mathrm{x}$-axis. . . . . . . 50

3.2 The sensing mechanism of the proposed sensor. (a) Illustrates the sensor under a vertical load of $500 \mathrm{~N}$. dz is the generated vertical displacement. (b) Shows the lateral displacement generated when $200 \mathrm{~N}$ of shear force along $\mathrm{x}$-axis is applied. .................... 52

3.3 The fiber-optic sensing principles for the simultaneous measurement of normal and shear forces in the $\mathrm{z}, \mathrm{x}$ and $\mathrm{y}$ directions. (1), (7). The transmitting and receiving fibers of y-axis force sensor. (2). Reflective surfaces. (3), (4). Z-Axis transmitting and receiving fibers. (5), (6). The transmitting and receiving fibers of the $\mathrm{x}$-axis. . . . . . . 53

3.4 Sensing principles. (a) occurs when a small amount of force is applied. (b) occurs when the applied force increases. . . . . . . . . . 55

3.5 (a) The developed test device that is mainly composed of ATI 6-axis F/T sensor and 6-axis stage. (b) The MTS experimental setup was utilized to apply pure uniaxial force to evaluate the cross-talk effects. . . . . . 56

3.6 Quadratic fitting model of force to voltage curves. (A), (B) and (C) are the fitting models of the $\mathrm{z}^{-}, \mathrm{x}$ - and $\mathrm{y}$-axis respectively. $\ldots \ldots . . .57$

3.7 Sensor response to the applied external force along the three normal axes using the MTS. . . . . . . . . . . . . . 59 
3.8 (a) The developed test device that is mainly composed of ATI 6-axis F/T sensor and 6-axis stage. (b) The MTS experimental setup was utilized to apply pure uniaxial force to evaluate the cross-talk effects. . . . . . 61

3.9 The experimental results of the $\mathrm{x}$-axis in a comparison to the acquired data from the ATI 6-axis $\mathrm{F} / \mathrm{T}$ sensor. The plot shows a second-order Output-Error, a third-order Auto-regressive-moving-average (ARMAX) and a second-order transfer function model corresponding to the real

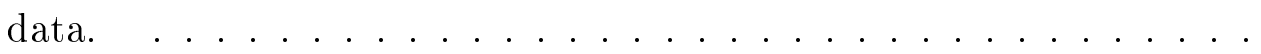

3.10 A comparison between the real data and the y-axis model. The graph demonstrates a second-order transfer function model compared to the

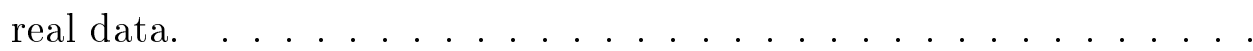

3.11 The similarity between the z-axis results using a second-order transfer function estimation model and the real data. . . . . . . . . . . . . . 64

3.12 The schematic block-diagram of the nonlinear Hammerstein-Wiener estimation model . . . . . . . . . . . . . . .

3.13 A comparison between Hammerstein-Wiener model of the x-axis and the real data. The fitting value was improved by $10 \%$ compared to the previous linear models. . . . . . . . . . . . . .

3.14 The optimized results of the y-axis using Hammerstein-Wiener model compared to the real data. . . . . . . . . . . . 68

3.15 A comparison between the Hammerstein-Wiener model used to model the $\mathrm{z}$-axis and the real data of the ATI 6-axis $\mathrm{F} / \mathrm{T}$ sensor . . . . . 
4.1 Structure of the proposed sensor. (1) Silicon rubber block. (2) Bottom plate (PLA). (3) Higher section to mount FlexiForce sensors. (4) Top plate. (5) LED and LTV holder. (6) TSL250R (LTV ). (7) OVLEW1CB9 (LED). (8) Fibre guider. (9) FlexiForce sensors (A301). (10) Top layer (11) Screw to precompression of the FlexiForce. (12) Optical fibres. (13). Light reflector. . . . . . . . . . . 78

4.2 The prposed sensor unit separated from the other related electronics. The LED and LTV are shown on the sensor side. . . . . . . . . 79

4.3 Sensors unit with top aluminum plate removed, exposing the FlexiForce sensors (covered with blue electrical tape for protection) $\ldots \ldots \ldots 81$

4.4 Two Sensor units are affixed on the force plate . . . . . . . . . . . 83

4.5 Ground reaction forces of the floor-mounted sensor using the least squares method and a neural network as calibration methods. (a) x-axis force (medio-lateral), (b) y-axis force (anterior-posterior), (c) z-axis force

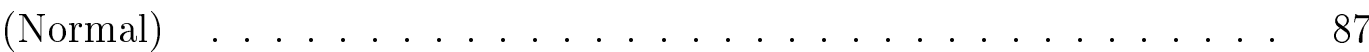

4.6 Ground reaction moments obtained from the floor-mounted setting using the least squares approach and a neural network as calibration. (a) $\mathrm{x}$-axis moment, (b) y-axis moment, (c) z-axis moment . . . . . 88

4.7 Sensor raw voltage and force applied $\ldots \ldots \ldots$. . . . . . . 89

4.8 Error between six-axis sensor and forceplate for least sqaures method . 89

4.9 Error between six-axis sensor and forceplate for neural network . . . . 90

4.10 Experiment set up for shoe-mounted sensors with two 6-axis sensor systems and 3 Optotrak rigid bodies (one not shown) $\ldots \ldots \ldots 90$ 
4.11 Ground reaction forces obtained from the shoe-mounted sensor using the least squares approach and a neural network as calibration. (a) x-axis force (medio-lateral), (b) y-axis force (anterior-posterior), (c) z-axis force

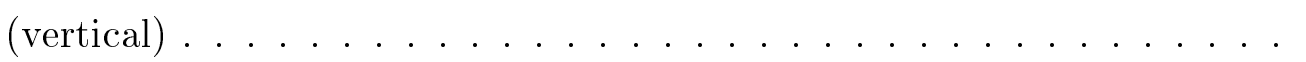

4.12 Ground reaction moments obtained from the shoe-mounted sensor using the least squares approach and a neural network as calibration. (a) $\mathrm{x}$-axis force (medio-lateral), (b) $\mathrm{y}$-axis force (anterior-posterior), (c) $\mathrm{z}$-axis force $($ vertical $) \ldots \ldots \ldots \ldots$

5.1 Structure of the proposed sensor. (1). Screw (2). Outer shield (3). Flat data cable (4). Printed circuit board for reliable connections (5). Top rigid layer (6). Light reflecting mirror (7). Compliant block (silicon rubber) (8). Optical fiber (9). LEDs and LTVs holder (10). LTV (Model: TSL-275) (11). LED (Model: OVLEW1CB9) (12). Bottom rigid layer. . . . . . . . . . . . . . . . . . . 104

5.2 Configuration of sensing elements. Each force and torque component has only one sensing element that contains LED, pair of fibers, LTV and reflective surface embedded along the top (A) and bottom (B) layers. 106

5.3 Prototype 6-axis fiber-optic F/T sensor in its assembled state. . . . . . 107

5.4 Sensor behavior due to applied torque around the x-direction. The top plate tilts which generates light intensity variation enabling $T x$

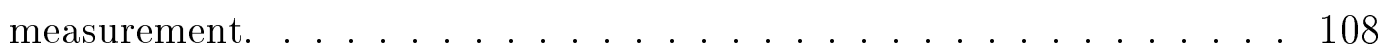

5.5 Sensing principle of the torque applied around the y-direction. The distance between the fibers tip and their respective reflecting mirror changes. This decreases or increases the intensity of detected light using

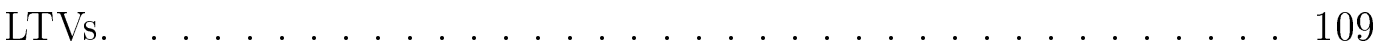


5.6 Torque $T z$ sensing mechanism. The top plate rotates with respect to the bottom plate and the torque is measured in accordance to the produced light intensity alteration. . . . . . . . . . . . . . . 109

5.7 Experimental setup for online data collection. The developed sensor is mounted to a standard 6 -axis $\mathrm{F} / \mathrm{T}$ sensor reference from Industrial Automation. Both sensors are connected to the same data acquisition system and data acquired using MATLAB, Real-Time Simulink for comparison. . . . . . . . . . . . . . . . . . . . . . . 114

5.8 Sensor response due to the applied normal-force in the z-direction. The multiple plots show a comparison between three different models and the reference data obtained from ATI 6-axis F/T sensor. The models include the novel Least Secures Decision Trees ( $L S D T)$, Least Squares Method $(L S M)$ only and Nonlinear Decision Trees NLDT alone. . . . 115

5.9 A comparison between three models: $L S D T, L S M$ and $N L D T$ of the $\mathrm{x}$-axis with the reference data. Forces different magnitudes, direction and frequencies were applied along the $\mathrm{x}$-axis $\ldots \ldots \ldots 116$

5.10 Experimental results of the developed prototype for forces applied along the $\mathrm{y}$-axis in a comparison between the estimated and actual data. . . . 117

5.11 Time-domain response of the proposed compliant 6-axis $\mathrm{F} / \mathrm{T}$ sensor due to applied torques around the $\mathrm{x}$-axis. A comparison between the estimated data using the proposed estimation models and the measured

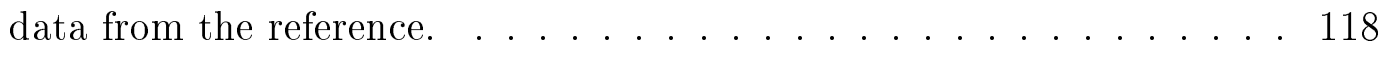

5.12 Response of the prototype under torques with different magnitudes and directions applied around the y-axis. . . . . . . . . . . . . 119 
5.13 Experimental results of the developed sensor for a comparison between actual data obtained from the reference and predicted data achieved from the sensor after applying three estimation models. The graphs demonstrate sensor performance under several torques around the z-axis. 120

5.14 Sensor characterization procedure of the novel calibration methodology named as Least Squares Decision Trees $(L S D T)$. Force and Torque data (voltages) acquired from the developed sensor are used as multi-inputs to two models: linear Least Squares model to estimate the applied forces and torques and nonlinear estimation model based on Decision Trees regression to estimated the errors. The sum of the two models is the novel $L S D T$ calibration approach. . . . . . . . . . . . . 120

5.15 The estimated sensor response vs the actual response of the reference for $F z$ estimators. (a) demonstrated the performance of the proposed Least Squares Decision Trees $(L S D T)$. Curves are not easy to distinguish because the model perfectly estimates data. (b) shows the output of the DT model only, which also shows an excellent predilection. (c) Presents the conventional linear $L S M$ model which exhibits slightly less accurate results. . . . . . . . . . . . . . . . . 121

6.1 Structure of the proposed fiber Bragg grating-based compliant normal force sensor. the sensor is composed of three parts: optical fiber with inscribed FBG sensing region, flexible sensing body and a rubber material used to fill the sensing body. . . . . . . . . . . . . 132 
6.2 Structure of the proposed fiber Bragg grating-based shear force sesning element. the sensor is composed of three parts: optical fiber with inscribed FBG sensing region,sensing body and a rubber used to fill the sensing body. . . . . . . . . . . . . . . 134

6.3 Illustration of the sensing mechanism of the proposed FBG-based force sensor using finite element analysis performed using Solidworks. . . . . 136

6.4 Strain distribution along the $\mathrm{x}$-axis due to the applied normal force. $\quad 137$

6.5 Explanation of the sensing mechanism and the strain distribution on the proposed FBG-based shear force sensing element using finite element analysis performed using Solidworks. As it can be clearly seen the optical fiber with the FBG is starched by a small element at the middle of the sensing body when a shear force is applied. . . . . . . . . . . . 138

6.6 The experimental testing steps which are used to test the proposed compliant FBG-based force sensor in both dynamic and static loading conditions. In the dynamic loading, arbitrary vertical forces with different magnitudes are applied on the top surface of the sensor whereas a different weights are used for static test. . . . . . . . . . . . . 140

6.7 The experimental results obtained from static loading conditions test. a linear function used to fit sensor data. . . . . . . . . . . . 141

6.8 The experimental results. The force applied in this case is much higher than the previous which generated in a higher deflection that results in slight nonlinearity. A quadratic function is used to fit the data. . . . . 143

6.9 The experimental setup used to test the proposed compliant FBG-based shear force sening element force. . . . . . . . . . . . . . 143

6.10 The structure of the proposed NARX prediction model model . . . . . 145 
6.11 The experimental results: A comparison between force measurements obtained from the ATI 6-Axis force and torque sensor and the results of the fabricated FBG-based normal force sensing element using the NARX model ............................ 146

6.12 The performance of the fabricated FBG-based normal force sensing elements when different amounts of loads (weights) are applied compared to the ATI 6-Axis force and torque sensor. . . . . . . . . . . . 147

6.13 A copmarison between the experimental results of the proposed FBG-based sensor and the force reference in real time. . . . . . . . . 147

6.14 The structure of the proposed linear regression with a nonlinear autoregressive exogenous (LR-NARX) calibration procedure. . . . . . . 148

6.15 The performance of the fabricated FBG-based normal force sensing element compared to the ATI 6-Axis force and torque senso when the novel LR-NARX model is used to characterize the sensor. The experiment results are perfectly matches the force reference. . . . . . . 149

6.16 A comparison between the performance of the novel LR-NARX and the performance of the static quadratic model. . . . . . . . . . . . 150

6.17 The performance of the fabricated FBG-based shear force sensing element compared to the ATI 6-Axis force and torque sensor. . . . . . . 151

6.18 The experimental results of the fabricated FBG-based shear force sensing element compared to the ATI 6-Axis force and torque sensor. The results matches the force reference. . . . . . . . . . . . . . 151

6.19 A comparison between cubic spline estimation model and a simple linear least squares method when a normal force is applied along the z-axis . 154 
6.20 The experimental results of shear force sensing element in a comparison between the linear calibration using least squares method and a cubic spline estimation model. . . . . . . . . . . . . . . 155

6.21 The response of the FBG sensing element. The generated wavelength variation due to the applied force . . . . . . . . . . . 155

7.1 A comparison between the actual force and the estimated data using a transfer function model and a state space model . . . . . . . . . . 163

7.2 Performance of the proposed dynamic models and their ability in characterizing the dynamic behaviours of the compliant force/torque

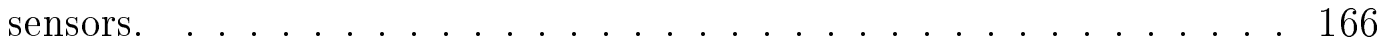

7.3 The experimental setup of the bandwidth test. A high frequency force is applied by hitting the propose sensor continuously generating high speed force. ......................... 167

7.4 The experimental results of the proposed sensor. (a) and (b) the performance of the $\mathrm{Fz}$ sensing element under a higher frequency force of the applied normal force in a comparison to the acquired actual data from the ATI 6-axis $\mathrm{F} / \mathrm{T}$ sensor when the linear least squares approach is used. . . . . . . . . . . . . . . . . . . . . 169

7.5 Compliant force and torque sensor response due to the applied shear force along the $\mathrm{x}$-axis using least squares optimization. . . . . . . . 170

7.6 Sensor response due to the shear force applied along the y-axis. . . . . 170

7.7 The experimental results when a linear least squares calibration is used to characterize the compliant force and torque sensor. This is a torque applied about the x-axis. . . . . . . . . . . . 171 
7.8 A Comparison between the performance of the compliant force and torque sensor calibrated using the simple linear least squares method and the actual torque data applied about the y-axis. . . . . . . 172

7.9 compliant force and torque sensor response due to the torque applied about the $\mathrm{z}$-axis in a comparison to the actual torque obtained from the ATI 6-axis force and torque sensor. . . . . . . . . . . . . . 172

7.10 The performance of the proposed Gaussian process regression models in comparison to the applied actual force.

1.1 floor-mounted experiment. Two three-axial force sensors are affixed on the Bertec force plate while the subjects walking across. . . . . . 237

1.2 Force sensor response due to the applied shear force along the $\mathrm{x}$-axis during gait $\ldots \ldots \ldots \ldots \ldots \ldots \ldots \ldots \ldots \ldots \ldots \ldots \ldots \ldots$

1.3 Force sensor response due to the applied shear force along the y-axis during gait . . . . . . . . . . . . . . . 238

1.4 Force sensor response due to the applied normal force along the z-axis during gait . . . . . . . . . . . . . . . 2 239

1.5 a comparison between the experimental results of the proposed 3-axis intensity based force sensor and the ATI force and torque sensor when a force is applied along the z-axis. . . . . . . . . . . . 240

1.6 Sensor performance when a force of high of high frequency and high speed is applied. . . . . . . . . . . . . . 240

1.7 The experimental results of the propse 3-xis force sensor and the reference sensor when a large amount of normal force is applied. . . . 241 
1.8 a comparison between the experimental results of the proposed 3-axis intensity based force sensor and the reference sensor in terms of shear force applied along the x-axis. . . . . . . . . . . . . 241

1.9 Shear force sensing element performance in a comparison to the ATI force and torque sensor. This shear force is acting along the y-axis. . . 242

1.10 Ground reaction force measurement. The sensor is tested during gait demonstrating sensor results of the shear force applied along the $\mathrm{x}$-axis

1.11 Ground reaction force measurement. The sensor is tested during gait demonstrating sensor results of the shear force applied along the y-axis

1.12 Ground reaction force measurement. The sensor is tested during gait demonstrating sensor results of the normal force applied along the z-axis 243

1.13 Experimental results of the proposed 3-axis force sensor during gait test presenting four steps while the shear force is applied along the x-axis. . 244

1.14 Ground reaction shear force acting along the y-axis presenting four steps of the sensor performance compared to the force plate. . . . . . . . . 244

1.15 Experimental results of the proposed 3-axis force sensor during gait test showing four steps while the normal force is applied along the x-axis . 245

1.16 One step test of ground reaction force along the $\mathrm{x}$-direction in comparison to the force plate measurements. . . . . . . . . 245

1.17 The experimental results of the proposed 3-axis force sensor in real gait measurement of the shear force of the y-direction. . . . . . . . . 246

1.18 One step test of ground reaction force along the z-direction in comparison to the force plate. measurements . . . . . . . . . 246

1.19 The experimental results of the rubber block due to the applied force. The material exhibits slightly nonlinear force-deflection relationship. $\quad 247$ 
1.20 The experimental results of the light-to-voltage converter due to the displacements generated as a result of the applied force which also exhibits a slightly a nonlinear behaviour. . . . . . . . . . 248

1.21 The final sensor response under the applied force. The sensor shows good linear correlation between force and generated output voltage. 249

2.1 Real-time actual gait measurement scenario. The experimental setup is composed of the proposed light intensity-based,6-axis fiber optic, force and torque sensor and a standard force plate. the sensor is affixed on the force plate and the subject walks across the it. . . . . . . . 251

2.2 Experimental results through a real gait measurement for the applied

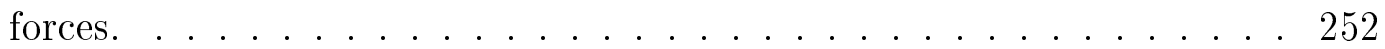

2.3 Experimental results through a real gait measurement for the applied

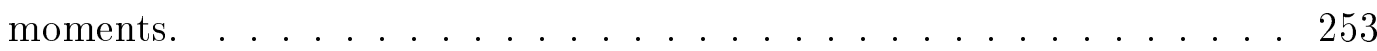

2.4 Experimental results through a real gait measurement . The dashed red line shows the estimated values of $\mathrm{F} / \mathrm{M}$ and using the proposed sensor an the blue line depicts the actual F/M measured by the force plate. . 254

2.5 Experimental results of the proposed 6-axis force sensor during gait test of six steps while the shear force is applied along the x-axis . . . . . 255

2.6 Ground reaction shear force acting along the y-axis showing six steps of the sensor performance compared to the force plate. . . . . . . 255

2.7 Experimental results of the proposed 6-axis force sensor during gait test of six steps while the normal force is applied along the z-axis . . . . . 256

2.8 Ground reaction moment acting about the x-axis showing six steps of the sensor performance compared to the force plate. . . . . . . . . 256 
2.9 Ground reaction moment acting about the y-axis showing six steps of the sensor performance compared to the force plate. . . . . . . . 257

2.10 Ground reaction moment acting about the z-axis showing six steps of the sensor performance compared to the force plate. . . . . . . . . 257

2.11 The transient response of the proposed second order compared to the third-order estimation models . . . . . . . . . . . . 258

2.12 The bode plot of the proposed second order and the third-order state space estimation models that shows a significant improvements in bandwidth of compliant force and torque sensor. . . . . . . . . . . 259

2.13 A comparison between the resulting output voltages. (a) shows the output voltage data recorded from the reference sensor. (b) demonstrates the resulting output voltage of the proposed sensor obtained from the optical sensing element. . . . . . . . . . . . 261

3.1 Experimental setup used for online force data recording. The developed sensor is attached to the ATI 6-axis F/T sensor reference from Industrial Automation. the proposed sensor is connected to si720 optical analyser and the refrence data acquired using Real-Time Simulink, MATLAB,

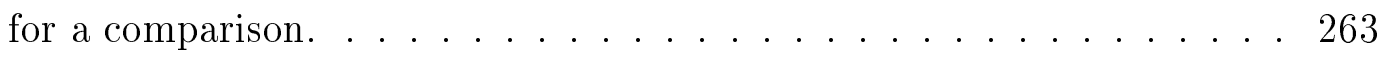

3.2 FBG integration system: optical sensing analyzer, Si720 Micron Optics. The system is used to detect the change reflected Bragg wavelength as a result of the applied force. . . . . . . . . . . . . . 263

3.3 the measured reflected power versus the generated wavelength for the fabricated fiber Bragg grating along the optical fiber. This strong resonance is referred to as Bragg resonance which is created due this particular FBG. . . . . . . . . . . . . . . 264 
3.4 Normal force sensing element response due to the applied normal force along the z-axis. A comparison between the performance of a simple linear least squares optimization and a spline function. . . . . . . 264

3.5 Normal force sensing element response due to the applied normal force along the z-axis using spline function as a calibration method . . . 265

3.6 Shear force sensing element response due to the applied shear force. The performance of the sensing element when a spline function is used to characterize the sensor compared to the actual force. . . . . . . 265

3.7 Shear force sensing element response due to the applied shear force compared to the actual force measured by ATI force and torque sensor. 266 


\section{List of Tables}

3.1 A summary of the cross-talk errors along each axis . . . . . . . . 60

3.2 The experimental results of the proposed 3-axis fiber-optic force sensor as a comparison to the real data of the ATI 6-axis F/T sensor. . . . . 70

4.1 Experimental results of 6-axis sensor mounted on floor during gait . . . 86

4.2 Comparison of triaxial sensor and six-axis sensor . . . . . . . . . . 91

4.3 The experimental results of 6-axis sensor attached to shoe during gait (applied force $) \ldots \ldots \ldots \ldots \ldots$

4.4 The experimental results of 6-axis sensor attached to shoe during gait

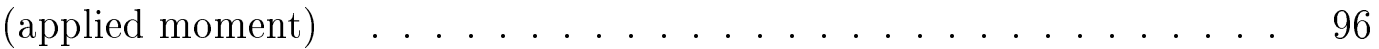

5.1 A summary of Experimental Results: Goodness of Fit, RMSE and Normalized RMSE for Each Estimation Model of Applied Forces . . 123

5.2 A summary of Experimental Results: Goodness of Fit, RMSE and Normalized RMSE for Each Estimation Model of Torques . . . . . . . . 124

5.3 Estimation Speed Comparison between The Novel LSDT Approach and DT Model Alone for Each Axis . . . . . . . . . . . . . . 125 
6.1 A summary of the experimental results: A comparison between the three proposed estimation models, LR-NARX, NARX alone and the linear least squares method alone in terms of the speed of prediction and the goodness of the fitting value. . . . . . . . . . . . . 152

6.2 A summary of the experimental results of shear force sensor: A comparison between the three proposed estimation models, LR-NARX, NARX alone and LR alone. . . . . . . . . . . . . . . . 152

7.1 A summary of the experimental results: A comparison between the three proposed estimation models, $2^{\text {nd }}$ order transfer function model , $2^{\text {nd }}$ order state space model and $3^{\text {rd }}$ order state space model in terms of the goodness of the fit, RMSE and Bandwidth. . . . . . . . . 164

7.2 A summary of the experimental results: A comparison between the three proposed Gaussian process regression models (GPR) estimation models, Rational-Quadratic GPR, Squared-Exponential GPR and Exponential GPR in terms of the speed of prediction and the goodness of the fitting value as well as the RMSE. . . . . . . . . . . . . . 174

7.3 A comparison between the proposed novel dynamic calibration methodology of the state space Gaussian process regression (SSGPR) and the GPR model alone in terms of the RMSE, the speed of prediction and the fitness value . . . . . . . . . . . 176 


\section{List of Acronyms}

$\begin{array}{ll}\text { Acronyms } & \text { Definition } \\ \text { FBG } & \text { Fiber Bragg Gratings } \\ \text { GRF } & \text { Ground Reaction Force } \\ \text { ABL } & \text { Advanced Biomechatronics and Locomotion } \\ \text { CMP } & \text { Centroidal Moment Pivot } \\ \text { ZMP } & \text { Zero Moment Point } \\ \text { COM } & \text { Centre of Mass } \\ \text { COP } & \text { Centre of Pressure } \\ \text { RMSE } & \text { Root Mean Square Error } \\ \text { NRMSE } & \text { Normalized Root Mean Square Error } \\ R^{2} & \text { Coefficient of Determination } \\ \text { MTS } & \text { Bionix Servo-hydraulic Test System } \\ \text { FSR } & \text { Force Sensitive Resistor } \\ \text { LTV } & \text { Light to Voltage Converter } \\ \text { TF } & \text { Transfer Function } \\ \text { ARMAX } & \text { Auto-Regressive Moving Average } \\ \text { LS } & \text { Least Squares } \\ \text { NLHW } & \text { Nonlinear Hammerstein-Wiener } \\ \text { OE } & \text { Output Error } \\ \text { DT } & \text { Decision Tree } \\ \text { LSM } & \text { Least Squares Method } \\ & \end{array}$




\section{List of Acronyms}

$\begin{array}{ll}\text { Acronyms } & \text { Definition } \\ \text { LSDT } & \text { Least Squares Decision Tree } \\ \text { ANN } & \text { Artificial Neural Network } \\ \text { SS } & \text { State Space } \\ \text { FRF } & \text { Frequency response function } \\ \text { GPR } & \text { Gaussian Process Regression } \\ \text { SSGPR } & \text { State Space Gaussian Process Regression } \\ \text { CLS } & \text { Compliant Limb Sensor }\end{array}$




\section{List of Symbols}

\section{Symbols}

$\Lambda_{m}$

$\theta_{n}$

$\theta_{m} / 2$

$\lambda_{U}$

$n_{e f f}$

$\Delta \lambda_{B}$

$\alpha_{f}$

$\Delta \varepsilon$

$\xi$

$P_{e}$

$\Delta T$

$p_{11}$

$p_{12}$

$\nu$

\section{Definition}

period of the etched patterns of the phase mask

incident angle of the ultra-violet beam

diffracted beam angle

ultra-violet wavelength

refractive index of the fiber

wavelength shift of the reflected spectrum

coefficient of thermal-expansion

strain variation

thermo-optic coefficient

elasto-optic coefficient

temperature variation

elasto-optic tensor 1

elasto-optic tensor 2

Poisson's ratio of the fiber 


\section{Chapter 1}

\section{Introduction}

\subsection{Motivation}

In the past decade, fiber-optic sensors have shown an immense potential for several modernized applications where high sensitivity, precision, miniaturization, weight and otherwise inaccessible measurements are the design essentials. Fiber-optic sensing techniques are capable of providing novel solutions for different sensing fields. They use an optical signal that propagates through an optical fiber cable made of silicon dioxide (fused silica). This provides high biocompatibility [23] which makes fiber-optic sensors an excellent sensing technology for biomechanical measurements. Conventional (and electronic) sensors utilized in biomechanical and biomedical applications are typically based on capacitive, strain gauge, piezoelectric or inductive sensing technologies. These sensors exemplify some profoundly sophisticated and widespread innovations, however, they exhibit several drawbacks too, including low flexibility, long-standing instability, fragility, inconsistencies, drift and heavy weight [24]. 
Obtaining precise force and torque information is crucial in applications such as biomechanics, ground reaction force and gait measurements, human-robot interactions and rehabilitation systems. Multi-axis force and torque transducers are the most favourable solution for such applications due to their strong ability to measure the complete degrees of freedom of an applied point force and torque. Although these multi-axis, force and torque sensors are important and have great measurement capabilities, they are also complex in their manufacturing and assembly processes. For example, the bonding procedure of strain gauges requires high precision. This makes it time consuming and therefore costly, specifically in labour costs [25, 26].

To obtain a suitable sensitivity for multi-axis, force and torque strain gauge-based sensors, researchers incorporate large numbers of sensing elements (strain gauges) distributed along the body undergoing deformation [27, 28]. This can cause communication bandwidth problems since a large data transmission between strain gauges and the main microcontroller is required, which can lead to unwanted lengthy delays in data transfer as well as higher power consumption. These shortcomings associated with the hardware components, rigidity and the size as well as their sensitivity to electromagnetic field interference undermine their functionality in clinical practices. In addition, standard traditional sensors are not desirable in medical centers where medical imaging techniques are used. Scanners that generate robust magnetic field and radio waves, such as in magnetic resonance imaging (MRI) scanners operate as antennae and produce significant errors with a penchant for heating impacts [29].

Fiber-optic sensing technology on the other hand, has significantly revolutionized the sensing industry as they are being progressively developed as a robust replacement. Fiber Bragg grating (FBG) technique in particular, is the most attractive and powerful fiber-optic sensing method due to its unmatched capacity for large scale 
multiplexing $[30,31]$. By implementing one of the commonly used FBG interrogation methods, Wave length Division Multiplexing (WDM), Time Division Multiplexing (TDM) or a combination [32-35], a large number of FBG signals can be acquired using only one detecting device along one optical fiber. In [36], as many as 33 FBG strain sensors were read using one optical spectrometer, presenting a measurement range of up to $500 \mu \varepsilon$ with a sensitivity of $1.2 \mathrm{pm} / \mu \varepsilon$. The commercially available spectrometer, Ibsen I-MON 512-USB, provides a high resolution FBG interrogation with wide dynamic range that can accommodate more than 70 in-line FBG sensors.

All of these characteristics make the fiber-optic sensors suitable to integrate onto the human body to measure strain and force distribution in applications such as ground reaction force measurements underneath the foot. A number of papers available in the literature showing an integration of fiber-optic sensing technology in biomechanics reveal that the technology is still novel [37], and it has not yet been commercialized.

The rapid population growth of the elderly and people with postural instability has increased the demand for effective healthcare accommodations. Developing human assistive technologies has become a growing market in the healthcare industry. The recent advancements in sensor technology and electronics have made assistive devices more and more plausible. It has been estimated that about one in three aged 65 years or older experience one fall a year at the very least [38, 39]. Fall predictions for the elderly and for people with gait instability are crucial in the prevention of fall-related injuries [40,41]. The reliable measurements of normal and shear forces on a foot are imperative for gait measurements in order to help maintain balance and prevent falls [42]. The assessment of the shear and normal forces between the ground and the foot can also be beneficial for those with diabetic foot ulcers [43, 44]. Studies have shown that over three quarters of amputation cases are attributed to foot ulcers 
$[43,45]$. Excessive plantar shear force is considered as the main factor behind diabetic foot ulcers [46]. Investigations in this area have been hindered by underestimating the influence of plantar shear forces and the lack of proper technology to measure them. It is only in the last decade that evidence can confirm the clinical consequence of shear forces in foot ulcers [47].

Either for improving human balance control or diagnosing diabetic foot diseases, a precise measurement for the total ground reaction forces is required. To capture the ground reaction forces, at least three main force components are required: vertical ground reaction force applied in the z-direction (or normal force) and two shear forces applied along the $\mathrm{x}$-axis ( medial-lateral force) as well as the y-axis (anterior-posterior).

Along with the assessment of the plantar normal and shear forces, ground reaction moments about the $\mathrm{x}-, \mathrm{y}-$ and $\mathrm{z}$-axes are required for balance control. Providing reliable gait measurements includes a postural sensing system with accurate detection of the total ground reaction forces and moments without disturbing the natural gait is desirable. Force plates are considered the standard measuring systems. They have the ability to accurately measure ground reaction forces and moments, but they are limited to a laboratory setting and clinical use. Moreover, the maximum number of steps that can be measured by a force plate are one or two per trial. Force plates are also incapable of measuring the forces and moments of each foot individually when both feet are on the same plate. Therefore, an ambulatory, wearable force and moment measurement system is more advantageous.

Ground reaction force and moment sensors for rehabilitation and biomechanical measurements must be mobile, lightweight and compliant so that it can not cause fatigue or discomfort for the elderly and for people with disabilities. Although ATI 
Industrial Automation's multi-axis, force/torque sensors can precisely measure the force and moment acting along the six normal axes, these sensors are metallic, strain gauge-based sensors which have low flexibility, large dimensions, and are heavy in weight and costly, making them unsuitable as a wearable, ambulant assistive devices. Another limitation in ATI sensors is the small moment measurements range in some models. It has been found that the applied moments particularly along $\mathrm{x}$-axis $(M x)$ can reach up to 40 N.m for a person weighing $50 \mathrm{~kg}$. Although ATI Mini85 SI-1900-80 model, for example, has sufficient moment range $(80$ N.m) for gait measurements, its weight is $0.635 \mathrm{~kg}$ with a thickness of $30 \mathrm{~mm}$ and the minimum number of sensors required for a reliable gait measurement are two per foot. Therefore, the total weight would be $2.45 \mathrm{~kg}$ of rigid instrumented shoes.

To address these limitations and deficiencies we present in this thesis, a novel solution with the development of multi-axis force and moment sensors based on fiber-optic technology providing significant improvements upon the current technologies of force and torque sensing systems. 


\subsection{Objectives}

The purpose behind this research is to develop various wearable, compliant, low-cost, and lightweight, multi-axis, force/torque sensors based on fiber-optic sensing technology that can be used in several biomechanical applications. One of these applications is ground reaction force and moment and gait measurements, both of which are the focus of this research. Fiber-optic force and torque sensors are currently being developed in the Advanced Biomechatronics Locomotion laboratory (ABL) at Carleton University to replace the previously developed wearable sensor system and to improve the sensing performance of the BalanceAid project. The current sensor of the BalanceAid was able to detect only the vertical ground reaction force based on force sensitive resistors (FSR) sensing technique with a relatively low precision. The objectives of this project are demonstrated as follows:

1. Developing several multi-axis force and torque sensors that can be used for application such as biomechanics and rehabilitation systems. Starting with the development of a 3-axis intensity modulation-based fiber-optic force sensor that can precisely measure the complete ground reaction forces and can withstand high normal forces with low cost. Detecting only normal force on a foot is not sufficient for gait measurements. For instant, the amount of forces generated during walking, for a person that weighs $60 \mathrm{Kg}$, can reach over $600 \mathrm{~N}$ and $100 \mathrm{~N}$ for normal and shear forces respectively [48]. In this case, the total ground reaction along z-, $\mathrm{x}$ - and $\mathrm{y}$-axis would be $812 \mathrm{~N}$ while the total shear forces in the $\mathrm{x}-$ and y-directions would be $141 \mathrm{~N}$. This means that, a $141 \mathrm{~N}$ out of $812 \mathrm{~N}$ (over 17\%) is ignored when measuring only the vertical normal force. This could significantly affect the precision of the measurement system. Such sensory information are 
needed to measure ZMP (Zero-Moment Point) and CMP (Centroidal Moment Pivot) [49]. Therefore, developing a sensor that has the ability to detect shear forces is essential in gait measurement.

2. The project is also seeking to overcome the inconvenient rigidity associated with the current available conventional sensors that are based on strain gauge technique. Furthermore, sensor weight is a huge concern especially for applications where a sensor is carried by a person for a real-time measurement. Sensors made of metallic materials that relies on strain gauge as their sensing technology such as ATI sensors are highly rigid and heavy. It is very desirable for a wearable sensor designed for biomechanics to be lightweight and compliant so that it would not alter the natural gait nor causing fatigue.

3. One of the primary objectives of this research is to increase the measurement capability of the proposed 3-Axis force sensor to be able to capture ground reaction moments in addition to the ground reaction forces. Center of pressure $(\mathrm{CoP})$ is very common in human postural analysis and balance control studies [50], and sensors do not have the ability to measure ground reaction moments cannot detect the center of pressure. Therefore, development of a 6 -axis $\mathrm{F} / \mathrm{T}$ sensor is key.

4. Minimization is another important goal in this project. Although both of the proposed 3- and 6-axis fiber-optic force sensors have a reasonable size as they have been used in a comfortable wearable scenario, the thickness $(1.2 \mathrm{~cm})$ can be reduced. Since theses sensors are based on intensity modulation, miniaturization is challenging because the fibers used to detect $F z, M x$ and $M y$ need to be permanently bent inside the sensing unit which requires from 3 to $4 \mathrm{~mm}$ of the 
total thickness. Therefore, another powerful fiber-optic technology named Fiber Bragg Gratings (FBG) is suggested to address this challenge. Using FBG-based technique, sensor thickness can be considerably reduced to a few millimeters.

5. An additional objective of this project is to reduce wires and electromagnetic interference effects. In the proposed intensity based fiber-optic sensors, the sensing element for each axis entailed three wires (LTV). Strain gauge-based sensors require four wires for each strain gauge. However, FBG-based sensor do not entail any wires as the sensing elements (FBGs) are inscribed along only one optical fiber. Also, the sensing element is optical rather than electric. This also provides better pressure distribution underneath a foot. Furthermore, in some 6-axis $\mathrm{F} / \mathrm{T}$ sensors such as strain gauge-based transducers, a huge number of strain gauges up to 32 are used to extract six components of force and torques. This means that the number of sensing elements exceeds the number of relevant six $\mathrm{F} / \mathrm{T}$ signal resulting in extra data transfer bandwidth and wires which can lead to extra delay in data transmission. In the current project, a 6 -axis $\mathrm{F} / \mathrm{T}$ sensor with only six force elements is a crucial goal.

6. Another main objective of this research is to develop a thin force sensor that can be easily integrated in any shoe and can accurately measure the plantar distributive force and moments as well as temperature beneath a foot using only one fiber with FBG-based sensing technique.

7. The intended applications of the proposed sensors are mostly incorporating dynamic locomotion with considerable displacement/strain, including gait analysis and ground reaction force measurements during walking and running as well as human-robot collision detection in robot movement. Compliant 
sensors, typically exhibit dynamic behaviours caused by the mass and viscoelastic properties of the flexible material used. In the proposed sensors, elastic materials used to obtain the required flexibility. Therefore, it is required for such class of sensors to compensate for the nonlinear behaviours natural to large displacement and strains. A nonlinear dynamic modeling and identification process and special calibration methodology is required to achieve a satisfactory sensor characterization.

8. An important goal is to performing human subject experiments to validate the developed sensing devices in their intended biomechanical applications such as in total ground reaction force and gait measurenets.

\subsection{Contributions}

Eight major contributions were accomplished during the course of this research listed as follows:

1. Develop a novel 3-axis, fiber-optic force sensor for biomechanical measurements: Designed, fabricated and calibrated a novel lightweight, compliant 3-axis force sensor based on light intensity modulation. Biomechanical applications such as human-machine interface, gait measurements and rehabilitation devices require sensing systems that are ambulatory, lightweight, flexible and can measure normal and shear force components. This sensor was able to measure the magnitude and the direction of the applied normal and shear forces simultaneously with a potential to adjust the measuring range.

2. Develop a novel, powerful hysteresis compensation methodology for compliant 
multi-axis force and torque sensors: Compliant sensors exhibit a delay phenomenon between the output and input. This is attributed to the complex mechanism in energy dissipation of the compliant materials. Therefore, modelling of such transducers and materials requires the ability to analyse the associated memory effects or the hysteresis. A novel hysteresis compensation method was developed showing a significant reduction in the generated hysteresis.

3. Development of a wearable, two-technology based 6-axis force and torque sensor for gait measurement: A compliant, multi-axis wearable force and torque sensor was designed, prototyped and characterized. The sensor combines two different sensing technologies: the fiber-optic force sensor and a pressure sensor matrix using FlexiForce transducers for ground reaction force and gait measurements. The sensor was used for the BalanceAid project as wearable ground reaction force sensor which was able to measure the total ground reaction force and moment during gait.

4. Developing novel calibration and sensor characterization strategies for compliant, multi-axis force and torque sensors: A novel calibration methodology named Least Squares Decision Tree (LSDT) was developed and applied, obtaining a high precision measurement of force and torque. The calibration procedure was split up into a linear regression part and a nonlinear regression part. The linear fit was performed using the standard linear least method (LSM) and the nonlinear regression part used a Decision Trees model (DT) to estimate the residuals. Both models worked as single calibration system, obtaining a high-quality performance of force and torque measurement.

5. Development of a lightweight, compliant 6-DoF fiber-optic based force and 
torque sensor: Designed, developed and successfully calibrated a novel, low cost, compliant 6-axis force and torque sensor based on fiber-optic technology. The sensor is lightweight and has a high force and torque measurement range up to over 1000 N. Due to its flexibility and wide force measurement range, the sensor can be used in different biomedical applications such as measuring the total ground reaction force, collision detection, human-robot interaction and applications where the compliance of the sensing element is desirable.

6. Experimental validation through an actual gait measurement scenario: Both the 3- and the 6-axis sensors were validated through an actual gait measurement scenario using two types of experiments. In the first experimental procedure, two sensor units are mounted on the Bertec force plate and the subject walks on them. In the second experimental set, the two sensors are attached to the bottom of the shoe, and steps are taken across the Bertec force plate. Optotrak markers are utilized to determine the orientation and position of the sensing units relative to the flour. Very accurate results were obtained in both sets showing the ability to measure the total ground reaction force and moment through a real application.

7. Development of compliant FBG-based, normal and shear force sensing elements for biomechanics: Designed, prototyped and characterized normal and shear force sensing elements based on light wavelength variation using fiber Bragg grating technology. The sensors are compliant, lightweight, small size and have high force measurement range. The performance of the sensing elements was evaluated through several experiments using static and dynamic loading tests. Both normal and shear force sensing elements have shown the ability to accurately measure the applied normal and shear force in real-time. 
8. Develop a novel calibration procedure for compliant FBG-based force sensors: Developed a novel calibration procedure that aimed to further enhance sensor performance and increase the speed of estimation. The calibration procedure is split into two estimation models: a simple linear regression (LR) achieved using least squares regression, and a nonlinear regression performed using nonlinear autoregressive with exogenous input (NARX) model. This calibration system named LR-NARX which was able to capture the nonlinearities attributed to the high compliance and large deflection.

\subsection{Outline}

The current thesis is an integrated work that incorporates six papers which are either published or awaiting review. This section provides an outline of the remaining chapters and papers included in this thesis.

Chapter 2: This chapter contains a literature review of a related work, current sensing technologies and methods. It gives an explanation of how fiber-optic sensing technologies are classified, functioned and used in biomechanical application such as in ground reaction force and gait measurement.

Chapter 3: The first paper (published in IEEE Sensors Journal in September 2017 [1]) covers the development process and calibration methods of a novel compliant, fiber-optic 3-axis force sensor based on intensity modulation for biomechanical measurements.

Chapter 4: The second paper ((published in IEEE Transactions on Instrumentation and Measurement in May 2018 [3]) covers the design, fabrication 
and calibration of multi-axis force and moment sensor that uses two different sensing technology for total ground reaction force and gait measurements. Experimental validation through a real gait measurement scenario is also included.

Chapter 5: The third paper (published in IEEE Sensors Journal in July 2018 [2]) covers the development and characterization of a novel intensity modulation-based, compliant, fiber-optic 6-axis force and torque sensor. It also covers an experimental validation through an actual intended application.

Chapter 6: The sixth paper (submitted to IEEE Sensors Journal in May 2019) covers the design, development and calibration of flexible, FBG-based normal and shear force sensing elements that operate based on wavelength variation.

Chapter 7: The fifth paper (submitted to IEEE Transactions on Instrumentation and Measurement in August 2019) cover a novel calibration methodologies for compliant force and torque sensors, showing how these novel calibration methods are effective in characterizing the complexity of the nonlinear dynamic behaviours of the compliant sensors.

Chapter 8: This chapter contains the conclusions of this thesis, recommendations and future work. 


\section{Chapter 2}

\section{Background and Literature Review}

This chapter introduces a review of the literature that is relevant to fiber-optic sensing technology and its classifications used for measuring the distribution of pressure and ground reaction force underneath a foot to achieve stability and maintain balance. This chapter also presents a literature review of how plantar normal and shear forces are measured using various fiber-optic based sensors with different fiber-optic techniques, demonstrating the importance of monitoring shear stress in diabetic foot ulcers as it helps to anticipate and prevent them from fall related injuries.

\subsection{Measurement Proficiency and Advantages of Fiber-Optic Sensors}

Fiber-optic sensors operate by transforming one or more characteristics of the light (eg. some characteristic like light intensity, phase or wavelength) that passes through its optical fibers. These sensors can be commonly subdivided into extrinsic or intrinsic 
sensors as seen in Figure 2.1. The extrinsic sensors guide the light to an external sensing region where the light signal is modulated through another medium. The different sensing mechanisms in extrinsic fiber optic sensors produce, transform, or modulate light rays, resulting in reflection, bending loss, wavelength or phase modulations. The light modulator may also include a gas or liquid cell which can be influenced by external perturbation, and hence altering the property of the light. In most cases, the fiber is only used to transmit optical signals from a sensing area to a photodiode followed by a data acquisition card. One advantage of this technique is the ability to access hard to reach places to perform measurements, thereby using non-contact sensing [51, 52]. In the intrinsic fiber-optic sensors, the light does not leave the fiber but remains within the wave guide. It modulates the properties of the optical signal inside the optical fiber to achieve the sensing functions by monitoring the resulting variations in the intensity, wavelength, and phase. Sensors that change the intensity of light inside the fiber are the simplest devices for sensing, as only one detector and light source are required. Intrinsic fiber-optic sensors provide spatial and continuous sensing along the full length of the fiber. They have the ability to measure a broad range of parameters such as pressure, strain, force, rotation, acceleration, displacement, acoustics, vibration, temperature and humidity [53] all using only one optical fiber. Thus, intrinsic sensors are much more attractive and are being extensively investigated in the field of sensing due to their high performance and multiplexing or multipoint measurements. Other advantages compared to the conventional electronic sensors are the small size, lightweight, flexibility and their resistance to electromagnetic interference. These sensors are also viable for inaccessible sensing areas, providing a substantial allowance for several applications. Fiber-optic sensor can operate under harsh conditions such as high temperatures, vibrations and shock. Another advantage 
to fiber-optic sensors is their large bandwidth and high sensitivity [54, 55].

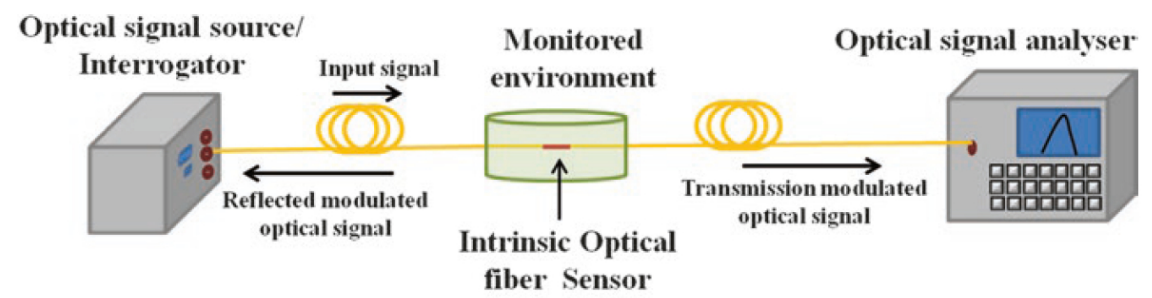

(a)

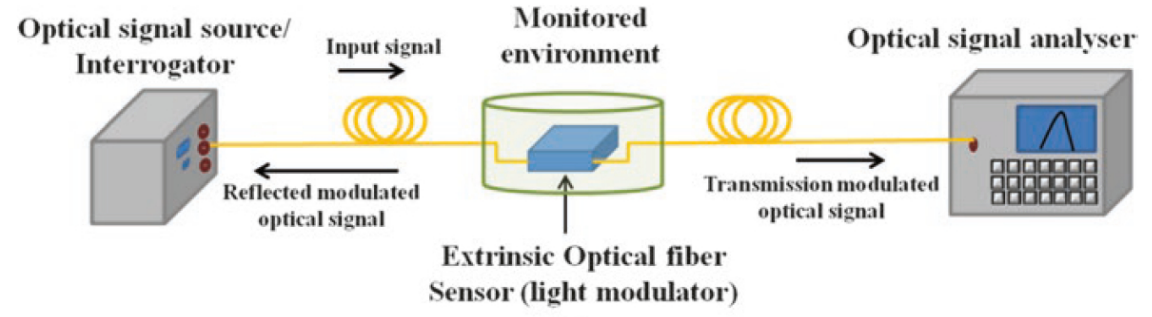

(b)

Figure 2.1: Fiber-optic sensors classification: (a) Intrinsic sensors (b) Extrinsic sensors [5].

\subsection{Strengths and Limitations of Current Biomedical and Biomechanical Measurement Systems}

Significant efforts in literature have been dedicated to the development of distributive plantar shear and normal force measurement systems based on various detection principles. Force-sensitive resistors (FSRs) are commonly used in plantar force measurements due to their simple interface and low manufacturing cost. In [56-59], a set of FSR sensors were integrated into flexible insoles to measure the distributed pressure of a foot. However, the prototypes were not capable of detecting the two components of shear forces in the $\mathrm{x}$ - and $\mathrm{y}$-directions. In addition, the material characteristics of FSRs are highly affected by temperature and nonlinearity. They are also fragile and have limited flexibity. Since the gait measurement involves bending 
during the walk, these sensors are not the best choice for this particular application. Capacitive-based and inductive-based transducers on the other hand, are broadly used in plantar pressure distribution measurements [60-62]. Although both of the capacitive and inductive techniques present an adequate precision and a wide range of pressure measurements, these types of sensors are generally susceptible to electromagnetic interference and temperature effects, and may experience drift and have small spatial resolution.

A piezoresistive-based plantar force sensor was reported for gait analysis measurements in [63]. The sensor exhibited a relatively high sensitivity and virtually invisible hysteresis effects, but had noticeable linearity and repeatability errors. These sensors, however, have the following drawbacks: considerably high stiffness, low breaking force, and the need for temperature compensator for each individual sensing element [64]. These sensors are mostly utilized for small strain measurements, and not designed for large deformations. In order to measure the pressure on a foot, an array of solid semiconductor sensors is needed, increasing the sensor's rigidity. In an attempt to overcome the inconvenient rigidity in [64], the sensor was embedded into elastic material, silicon rubber.

Piezoelectric-based sensors are also widely used in plantar force measurements [65, 66]. An electric charge is generated when the piezoelectric material experiences an external force. Electric polymer foils are the most extensively used material in biomedical applications such as polyvinylidene fluoride (PVDF) and cellulose nanofibril (CNF) [67], which can be made very thin (between micrometers and millimeters in thickness), making them a potential solution for many applications[68]. A flexible, piezoelectric-based polymer foil sensor made of polyvinylidene fluoride was presented in [69] to assess the distribution of foot plantar pressure. Since the proposed material 
is highly sensitive to pyroelectricity (i.e. the material generates a voltage when there is a change in temperature) [70], a temperature compensator was necessary to mitigate the pyroelectric effects. In addition, the data acquisition system is complicated and the sensor was not capable of measuring the shear forces in the $\mathrm{x}$ - and $\mathrm{y}$-directions. Moreover, the materials were structurally fragile and likely to get damage during common use [71].

All of the aforementioned sensors are micro-electromechanical systems (MEMS) technology, making them vulnerable to electromagnetic fields and perturbations caused by its surroundings. Meanwhile, fiber-optic sensors are comparatively better candidates due to their immunity to electromagnetic interference, small size, lightweight and wide measuring range. The most common sensing methods of fiber-optic sensors in biomechanical and biomedical applications are mainly based on light intensity, wavelength and phase modulation.

\subsection{Intensity Modulated-Based Fiber-Optic Sensors}

Intensity based sensors are inherently one of the primal types of fiber optic sensors. In the intensity modulation structure, the optical signal is transmitted through optical fibers, then modulated. There are several techniques for intensity modulated fiber-optic sensors based on the different mechanisms, such as transmission loss, reflection, shutter modulation and fiber displacement. The special advantages of intensity modulated sensors are their simple signal conditioning circuit and detection system. They are straitforward in fabrication and low-cost with high-quality performance [72, 73]. 


\subsubsection{Microbending-Based Fiber-Optic Sensors}

Microbending is one of the most attractive intensity modulation techniques. it involves tilting the fiber to induce light intensity losses as a result of a change in radius of curvature. Microbending sensors have been utilized in applications where the sensing parameter such as force, strain, position, pressure, and acceleration are mechanically attributed to the displacement of a mechanism that deforms the fiber. As the deformity increases, the loss of radiation increases and the transmission of light decreases as a representation of the external perturbations acting on the fiber [74, 75].

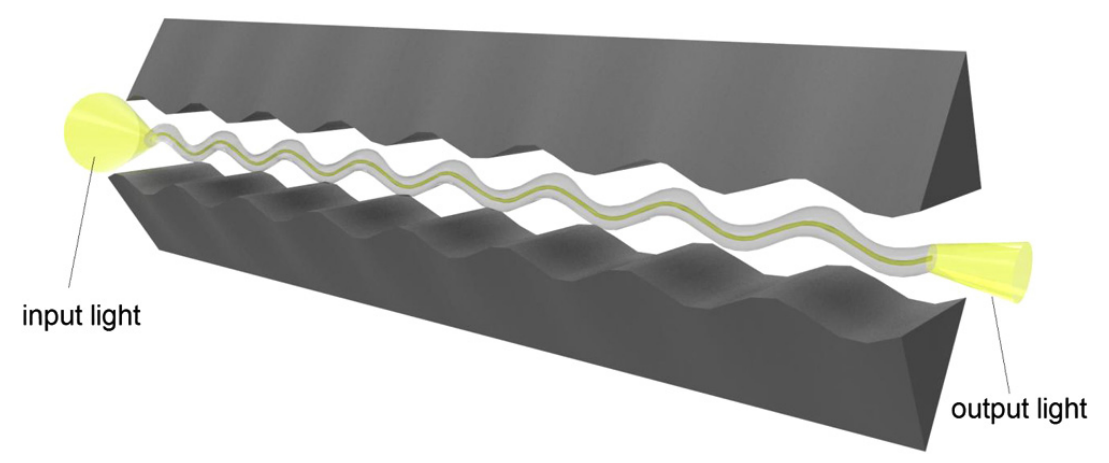

Figure 2.2: Radiative losses as a fiber undergoes bends [6].

The development of lightweight, compact and compliant force sensors that have a wide measuring range and low energy consumption are very ideal for applications in biomechanics and rehabilitation systems. The collection of the ground reaction force, plantar pressure, and body kinematics are broadly utilized to characterize the normal and abnormal behavior of the human foot in gait analysis. These analyses work towards diagnosing inferior limb problems [76], maintaining balance for the elderly and for people with disabilities [77], monitoring force distribution beneath the foot in a stance phase [78], and preventing injury [79]. More recently, plantar 
pressure distribution has also been designed for human identification [80] as well as for rehabilitation purposes [81, 82]. The commercially available plantar force measurement systems or those under development in laboratories for research, they meet specific requirements for different applications. As such, the shape and configuration of the sensor (or its design) varies in accordance with its application. For the purpose of this research, the sensor design would include in-shoe measurements such as pressure distribution, and total ground reaction force and moment for gait measurements. The design requirements in constructing plantar force sensors are accuracy, spatial resolution, sensitivity, sampling frequency and reliable calibration [83].

In-sole and out-sole plantar pressure sensors have enhanced efficiency, mobility, flexibility and a relatively low cost for pressure measurement systems in biomechanics. More importantly, for devices to be fully flexible and wearable while observing all activities on a daily basis, the device ought to be wireless and it ought to have a relatively low electrical power consumption. The practical solution for short wireless application range is to use inexpensive devices such as an on-chip antenna. The on-chip antenna allows devices to work at low frequency bandwidths while adding little to the size of the device; $[84,85]$ show examples of its use.

Reliable ground reaction force measurements, including shear and normal force components, are required in order to investigate the role that shear and normal forces play in producing plantar ulceration in the case of a diabetic patient [7]. A high resolution, flexible force sensor has been fabricated based on intensity loss through bending. The prototypes in $[7,86,87]$ have the ability to measure the pressure distribution underneath a foot. These optical fiber sensors for this prototype are configured to incorporate two pairs of optical fibers, each pair comprising two parallel optical fibers and each pair configured perpendicular to each other, as illustrated 
in Figure 2.3. The four resulting intersections provides a means to assess the shear force by analyzing the set of misalignments of four intersections when applying a shear force. Normal force is measured through the intensity attenuation as a result of bending the optical fiber.

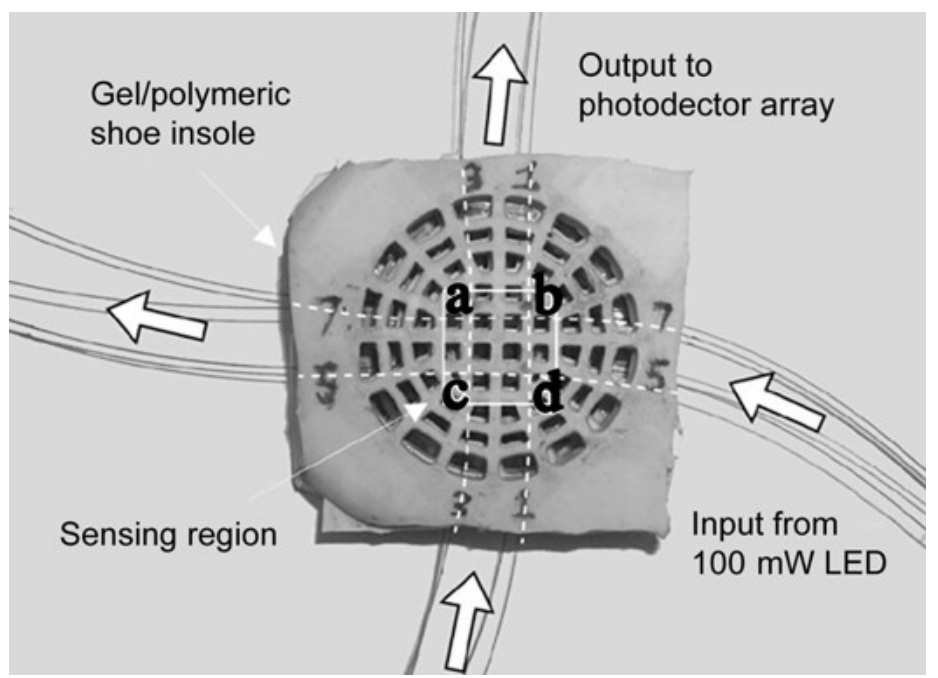

Figure 2.3: plantar and shear pressure sensor prototype [7].

Furthermore, this prototype is not able to determine forces applied simultaneously at more than one intersection, particularly when the number of output signals from the fibers are less than the number of measured pressure points (sensors). In [8, 88], the sensor was fabricated so that the mesh of optical fiber is embedded into one polydimethylsiloxane (PDMS) sheet as shown in Figure 2.4. This prototype uses, in addition, a neural network imaging algorithm to identify the load shapes and to determine the corresponding magnitude of the applied loads.

Biomedical and biomechanical sensors are inclind to construction challenges and specific problems related to the interface between human biological organs. Such sensors should be safe, reliable, biocompatible, highly stable, lightweight, and agreeable 

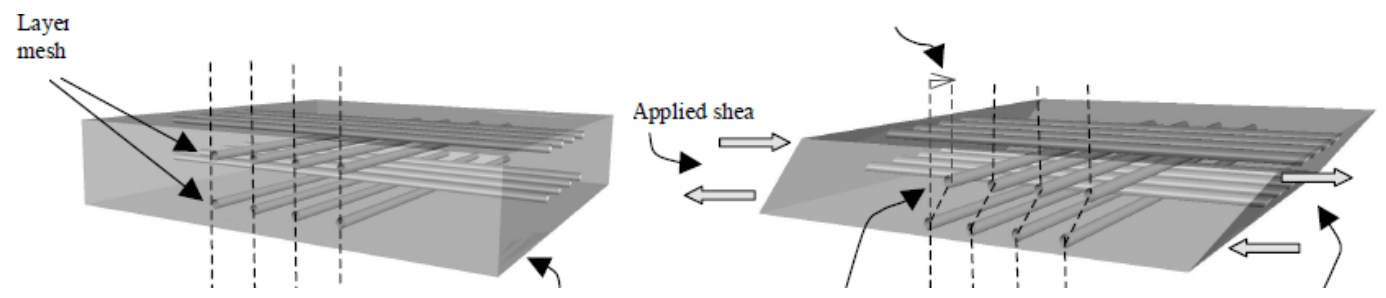

Figure 2.4: plantar and shear pressure sensor prototype [8].

to sterilization. Moreover, they must be cost-effective and have a low environmental impact. Device packaging is a specifically critical aspect as these sensors must be small, compact and wearable to be applied in clinical practices [89].

A flexible insole ground reaction force sensor is reported in [9]. The sensor working principle relies on the light intensity change that is proportional to the optical fiber's angle of curvature. The change in the optical fiber curvature occurs as a result of the applied force, which leads to an optical power attenuation (bend loss). As shown in Figure 2.5, the sensor consists of five sensing regions inscribed along a single optical fiber where one end is connected to light emitting diode and the other is connected to a photodetector. To increase the power attenuation (sensor sensitivity), the optical fiber is modified by eliminating the cladding to facilitate fiber bending and therefore increase the angle of curvature. The optical fiber is mounted to an insole made from deformable material to enhance sensor flexibility.

Although the proposed sensor shows satisfactory results, it is only able to measure one component of ground reaction force which is the vertical ground reaction force. Moreover, the number of the force sensing elements or regions undergo bending (five sensing elements) exceeds the number of the sensing channels (one channel). This means that only one force single is acquired even though five in-line sensors are used.

Bend loss sensors have been clinically utilized in health monitoring applications 

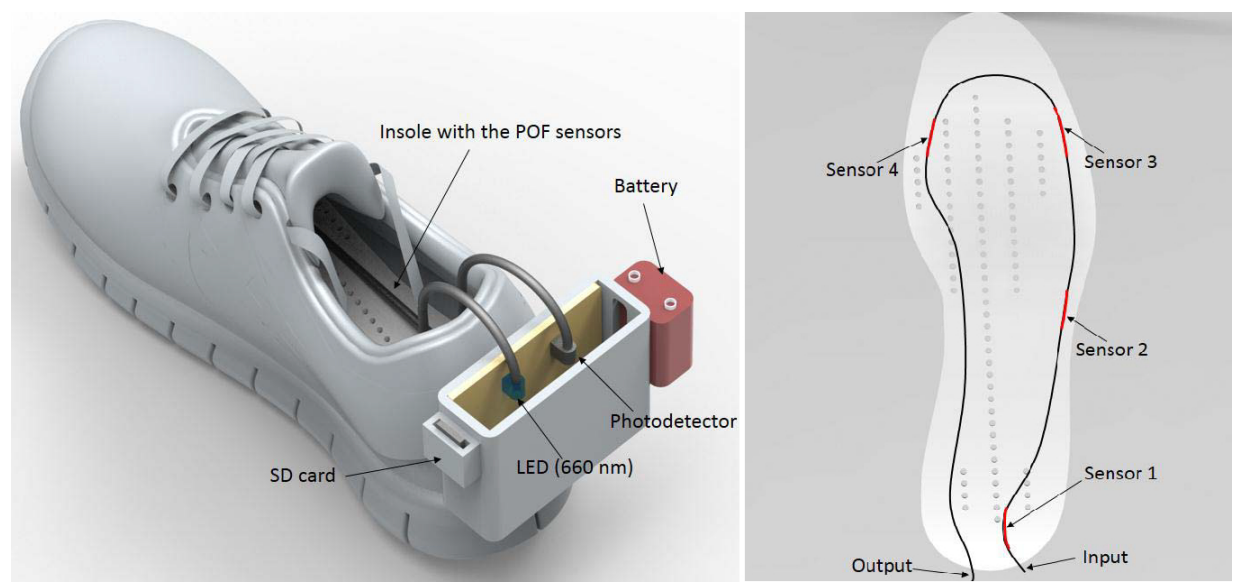

Figure 2.5: Pressure insole developed based on bend loss sensing technique [9].

including human lower and upper limbs movement tracking, curvature of lumbar spine, gait posture, heart and respiration rate assessments. In the upper limb montion monitoring, the assessment of the angular movement of finger, arm, wrist, elbow as well as the shoulder joint is essential. A wearable fiber-optic sensor based on macrobending technology was developed to measure the angular movement of the upper human articulations. The optical fiber was installed on a polychloreprene (PCP) rubber suit where the fiber can only bend at the joints of the upper limbs (wrist, elbow and shoulder). Taking advantage of the light attenuation, the movements of the arm can be monitored from the bending fibers [90].

\subsubsection{Reflection Based Fiber-Optic Sensors}

Reflection based intensity modulation fiber-optic sensors are ideal in the field of sensing due to its simple sensing principles that rely on the intensity alteration in the reflected light. The reflecting technique incorporates a moveable reflective surface positioned in front of fibers, one transmitting and one receiving. The reflective surface moving in a longitudinal direction leads to an axial displacement that could be 
measured by detecting the light intensity variation using photodetectors [91].

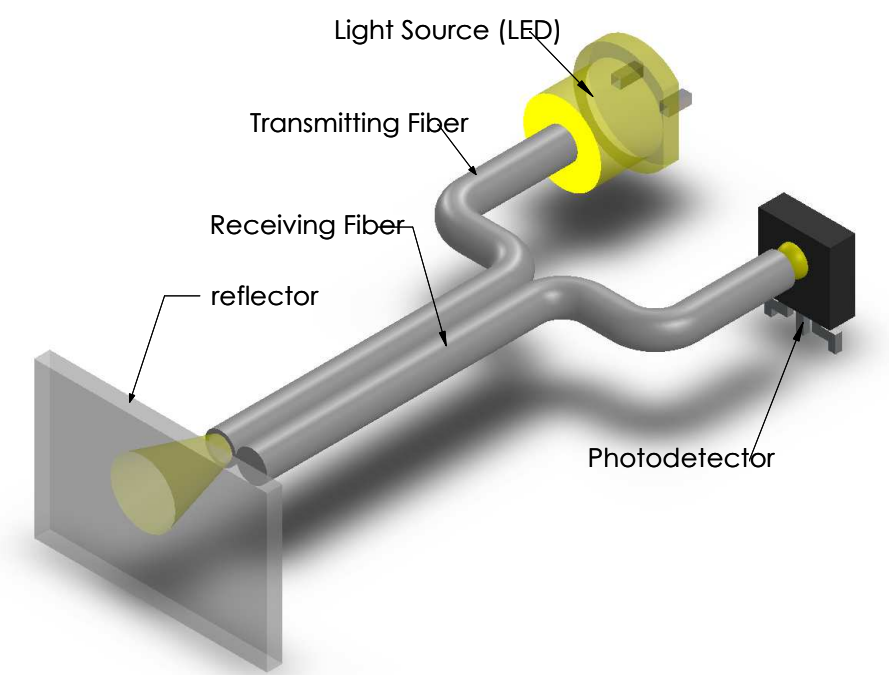

Figure 2.6: Schematic of an optical fibers located in the front of a movable reflecting surface. The intensity of the reflected light increases when the distance between the tip of fibers and the reflector decreases.

Figure 2.6 illustrates two optical fibers are working as one optical sensor. The light beams are guided through the transmitting fiber using a light source such as an LED, and reflected by the movable mirror at the other end. The distance between the tip of the fibers and the reflective plate corresponds to the magnitude of the measurand. The reflected light gets conveyed through the receiving fiber to a detector, where its intensity is dependent on the distance between the fiber tip and the reflective surface. An increment in the distance causes a decrement in the intensity. Figure 2.6 shows the working principles that enable the sensor to measure various physical variables such as force, pressure, temperature, displacement, etc. [92]. 


\subsubsection{Reflecting-Based Minimally-Invasive Surgical Force Sensors with Catheter-Tip}

Accurate measurement of the displacement is crucial since parameters like force, pressure and strain are operatively related to the variation of the displacement. Intensity modulated based sensors with movable reflective surfaces located at the sensor tip have been widely used in minimally-invasive surgery and cardiac pressure measurements. In [11], a flexible, single-axis catheter was developed to assess the intracardiac pressure in heart surgeries. Three pairs of fibers (three transmitting and three receiving fibers) were used to detect the deformation generated when pressing on a heart. Due to the nonlinear characteristic response of the elastic material used and its hysteresis, a viscoelastic calibration is necessary to better estimate the applied force. Although the sensor showed good measuring performance, it was not able to measure shear forces. The absence of detecting the two components of shear force makes the sensor lacking as a whole.

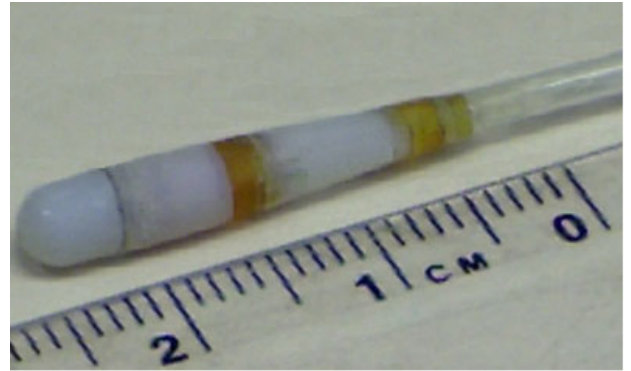

(a)

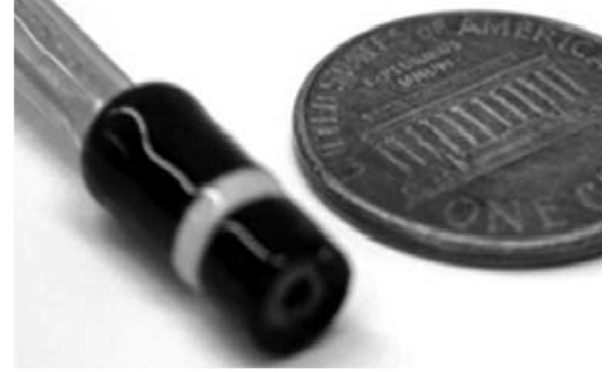

(b)

Figure 2.7: Intensity-modulated reflected light force sensor catheters (a) having triaxial sensing with three optical fibers and (b) having uniaxial sensing with six optical fibers $[10,11]$.

To overcome this challenge, a triaxial catheter with high sensitivity force sensor is 
presented in [93]. This prototype uses only three fibers to measure forces with each fiber acting as transmitter and receiver at the same time, using a splitter at the end of each fiber. The resulting plots of the dynamic test revealed that the phase of the calibrated signal of the proposed sensor did not match the actual signal. This is due to the hysteresis effects that cannot be captured with conventional linear calibration methods. Overall, both sensors are susceptible to the loss of light due to bending and therefore both have erroneous readings from the lack of hysteresis modeling in the calibration process. Figure 2.7 shows both prototypes.

\subsubsection{Hand-grip Force Sensor with MRI Compatibility Based on Light Reflection}

The magnetic resonance imaging (MRI) technique has been used extensively to provide high spatial resolution for noninvasive procedures in the investigation of living tissues. However, one of the major restrictions of these imaging systems is that any sensors used inside an MRI scanner must be resistant to electromagnetic fields and must be magnetic resonance compatible [94]. Fiber-optic sensors are the most attractive technology that is suitable for such applications compared to the conventional electric force sensors such as strain-gauges which emit electro-magnetic waves that result in low signal-to-noise ratios (SNR). This can negatively affect the functionality of an MRI system. A hand-grip fiber optic force sensor was reported in[13]. The sensor was designed based on intensity-modulated reflected light to investigate human motor-control during MRI scanning. Two fibers were used, one for transmitting and one for receiving the reflected light. The sensor was made of rigid aluminum-A5052. The results of this sensor showed good linearity and no observable hysteresis effects, but the sensor had a limited range of $50 \mathrm{~N}$ and the material is inflexible and heavy, making it ineffective for this application. 


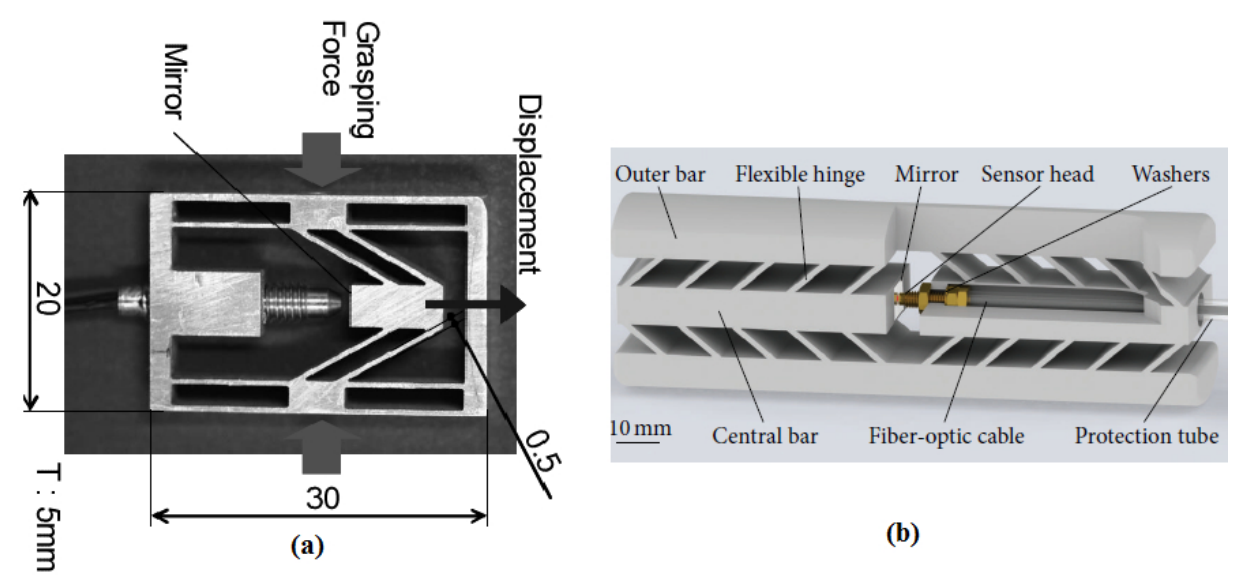

Figure 2.8: Hand-Grip Fiber-Optic Force Sensors. (a) metal elastic body and (b) the $3 \mathrm{D}$ printed body structure [12, 13].

In [12], a reflection-based fiber-optic sensor used to measure gripping force is 3D printed using Polylactic Acid (PLA). This sensor was lightweight and flexible with a higher measuring range (from $0 \mathrm{~N}$ up to $800 \mathrm{~N}$ ). The experimental results have confirmed that the sensor exhibits small hysteresis and small nonlinearity errors only in the range of $0-500 \mathrm{~N}$. The sensor also exhibits creep errors, with a recovery process that lasts for one hour and never recovering its full shape. The creep error was $26.5 \mathrm{~N}$ for just one grip of thirty minutes. This sensor is not durable as the recovery errors could increase overtime. Overall, in both sensors shown in Figure 2.8, the developers did not take into account the bend loss effects. These sensors are susceptible to bending during grip force measurements since the distance between this sensor handle and its electric components is relatively long. This distance is essential for this sensor to avoid electric noise emissions, which in turn can disturb medical equipment such as an MRI scanner. 


\subsubsection{Shutter-Based Intensity Modulation Fiber Optic Sensors}

In the fiber-optic displacement sensors, at least one pair of transmitting and receiving fibers is used to detect displacement [6]. These fibers positioned opposite to each other, with one affixed at one end and the other is free to move, such that the variation of the distance between the fiber tips generates altering light intensity, received by a photodetector at the other end of the receiving fiber as shown in Figure 2.9 (a).

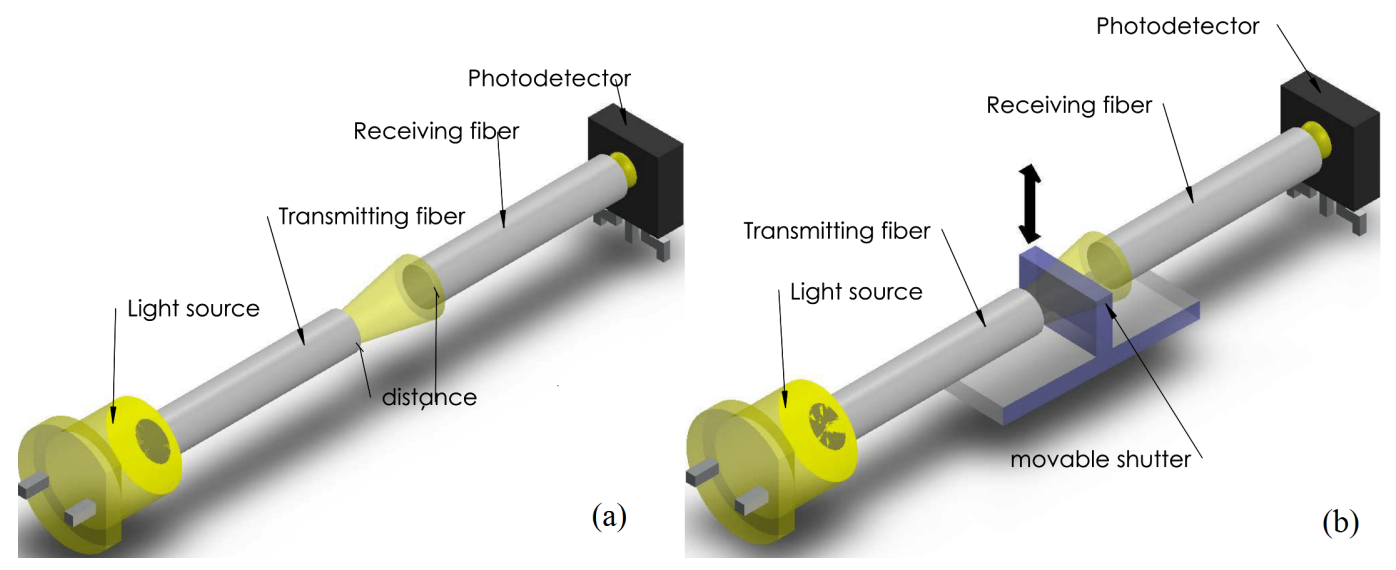

Figure 2.9: Fiber-optic displacement sensors. (a) Measure the displacement directly using two opposite fibers. (b) a moveable shutter is used to modulate the intensity of light to detect the displacement change.

In the shutter-based modulation technique, the sensing mechanism incorporates a movable shutter in between the transmitting and the receiving fiber, as depicted in Figure 2.9 (b), which operates as a light barrier. The fibers in this method are fixed and the shutter is free to move vertically to detect the vertical force/displacement or longitudinally to measure the lateral force/displacement. The shutter increases or decreases the light transmission between the pair of fibers which alters the intensity of the light during the shutter movement due to the external applied force. 
The shutter modulation method has been used in the developments of distributive plantar normal force measurement but without the use of fibers. An insole pressure sensor was proposed and prototyped in [14] and [95]. A set of LEDs and photodetectors were integrated inside a flexible insole. Each LED directly faced its respective photodetector. A small element acting as a shutter in the insole prevents the light transmission from LEDs to photodetectors, allowing the normal pressure measurement based on the light intensity attenuation, as shown in Figure 2.10.
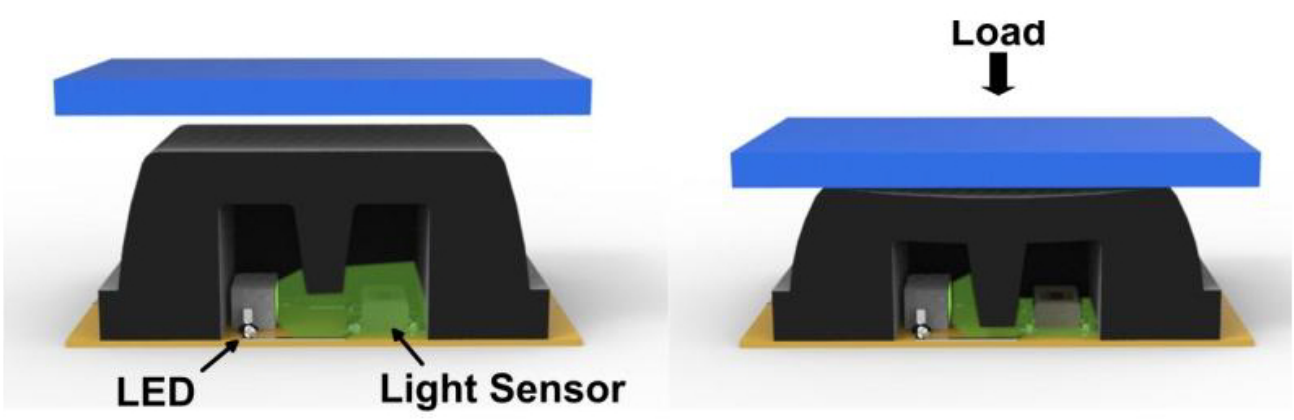

Figure 2.10: Optoelectronic sensing element without the use of optical fibers to measure the vertical ground reaction force [14].

However, this sensor was not able to measure the shear forces along the $\mathrm{x}$ - and y-axis which are important force components in gait analysis. Only the applied normal force along the $\mathrm{z}$-axis was detected.

\subsection{Wavelength-Modulated Fiber-Optic Sensors}

Wavelength or spectral modulation techniques use the variation of the wavelength of the light as its sensing pinciples. Any external perturbation that occurs in the measurand will generate a shift in the wavelength of the optical signal. There are three types of wavelength-modulated sensors such as Fiber Bragg gratings sensors 
(FBG), Fluorescence sensors and Blackbody sensors. FBG based fiber-optic technique is the most common and extensively used method in the development of fiber-optic sensors and will therefore be the focus in this section. As a distinctive class of fiber-optic sensors, FBG sensors have universally attracted a significant attention in different research fields. Unlike conventional sensors, FBG-based sensors are electrically passive, nonconductive and invulnerable to electromagnetic interference or light source fluctuations. Furthermore, FBG sensors are lightweight, small size, and they can withstand harsh environmental conditions with no temperature drift. Their multiplexing and distributive sensing capabilities make it feasible to accommodate multiple sensing points in only one single fiber, detecting several parameters such as strain, temperature, pressure, force, vibration. etc. All of the previously stated advantages make the FBG sensors a potential solution for different applications [96-98].

Ever since the FBG technology was first explored for detection purposes over the last three decades, the FBG sensors have been rapidly developed for practical applications such as biomedical and biomechanical measurements [99], structural health monitoring (SHM) in civil engineering [100], mechanical instruments [101] and aerospace engineering [102].

\subsubsection{FBG Fabrication}

Fiber Bragg gratings are formed by creating a permanent periodic variation in the reflective index along the optical fiber core in the direction of optical signal propagation. The periodic, cylindrical-shaped objects inscribed along the fiber core are called Bragg gratings $(\mathrm{BG})$ which operate as a wavelength filter. Bragg gratings can be fabricated by using two common methods: holographic technique [103] and phase mask technique 
[104]. In both methods BGs are created by exposing an optical fiber to an intense ultraviolet (UV) beams. For holographic method, a laser beam is transmitted through a splitter to produce two laser paths which are then combined and intersected inside the fiber, generating permanent regions along the fiber core called Fiber Bragg Gratings (FBG) that have different refractive indices. In this method, the BGs wavelength can be controlled by adjusting the angle between the intersecting laser beams [105].

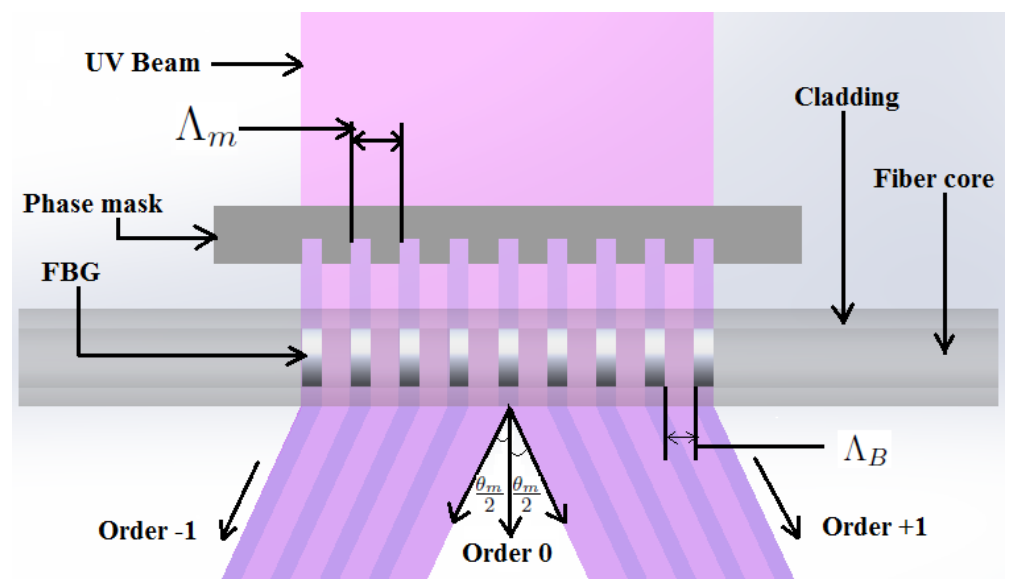

Figure 2.11: Model FBG theory

For the phase mask approach, a single laser beam and a phase mask are used to produce BGs, creating an interference pattern along the fiber core as shown in Figure 2.11. The phase mask is usually made of fused silica with etched periodic patterns which is used to split the single laser beam into various diffractive orders which then interfere and typically overlap each other in the fiber. This interference generates the required FBGs in the fiber core, whereas the period of imprinted gratings is either half or equal to the period of the phase mask, depending on the exposure geometry. The operating principle of the phase mask is based on the diffraction of the incident UV light into several equal orders: $m=0, \pm 1, \pm 2$, etc. The general equation of the 
diffraction is described as the following:

$$
\Lambda_{m}=\frac{m \lambda_{U}}{\sin \left(\frac{\theta_{m}}{2}\right)-\sin \theta_{n}}
$$

where $\Lambda_{m}$ is the period of the etched patterns of the phase mask, $\theta_{n}$ is the incident angle of the UV beam, $\theta_{m} / 2$ is the diffracted beam angle, and $\lambda_{U}$ is the wavelength of the UV beam. When the UV beam is radiated at the normal incident of $\theta_{n}=0$, the diffracted beam is split into three orders of $m=0$ and $m= \pm 1$. Thus, the diffraction equation becomes:

$$
\Lambda_{m}=\frac{\lambda_{U}}{\sin \left(\frac{\theta_{m}}{2}\right)}
$$

The period of the etched patterns of the phase mask, $\Lambda_{m}$, is adjusted according to the required Bragg wavelength $\lambda_{B}$ for the gratings inscribed along the fiber core. The gratings periodicity $\Lambda_{B}$, or effectively the distance between two gratings, can can be described by the following equation :

$$
\Lambda_{B}=\frac{\lambda_{U}}{2 \sin \left(\frac{\theta_{m}}{2}\right)}=\frac{\Lambda_{m}}{2}
$$

The equally diffracted orders \pm 1 are intersected inside the fiber, which creates the FBG interference patterns that have grating periodicity of half the period of the etched patterns along the phase mask.

\subsubsection{FBG Sensing Principles}

When an incident spectrum of light propagates through the fiber gratings, a certain wavelength named Bragg wavelength $\lambda_{B}$, is reflected back while the rest of the spectrum is transmitted unaffected as can be seen in Figure 2.12. The FBG structure functions 
as a wavelength-selective filter. The reflected Bragg wavelength is determined by the product of the effective index of the core mode propagating in the fiber $n_{\text {eff }}$, by twice the grating period, $\Lambda_{B}$.

$$
\begin{gathered}
\Lambda_{B}=\frac{\Lambda_{m}}{2}=\frac{\lambda_{B}}{2 n_{e f f}} \\
\lambda_{B}=2 \Lambda_{B} n_{e f f}
\end{gathered}
$$

Equation (5) shows that the reflected Bragg wavelength, $\lambda_{B}$, relies on the refractive

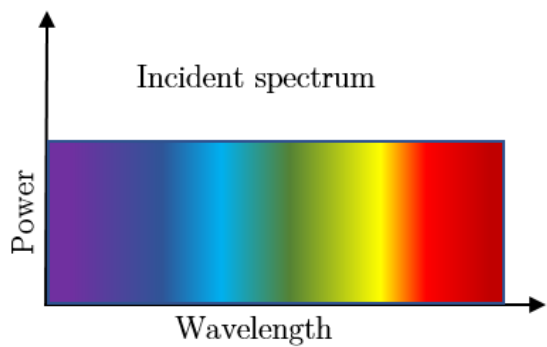

Wavelength

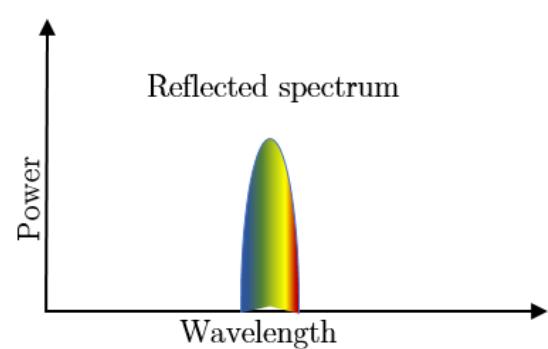

Wavelength

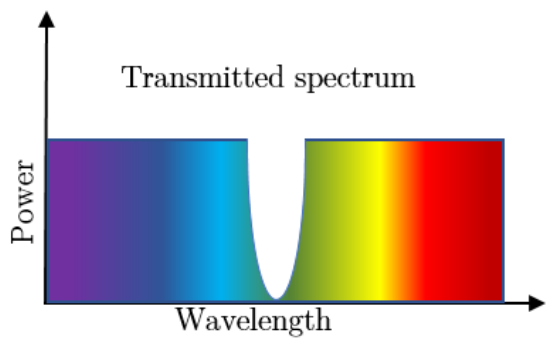

Figure 2.12: Light propagation through the FBG sensing element. A narrowband of the transmitted light with specific wavelength reflects back due to the Bragg gratings.

index of the fiber core and the inscribed grating periodicity along the fiber. When an external perturbation that can alter the periodicity of the grating is applied such as an axial strain as shown in Figure 2.13, the FBG responds accordingly and causes a proportional shift in the reflected Bragg wavelength. With a real-time calibration 
process, strain values and many other parameters can be dynamically measured. The FBG sensing technique exhibits a highly linear relationship between the reflected Bragg wavelength and the measurand, which is limited to the elastic deformation range of the fiber. FBG-based strain sensors can be employed indirectly to measure force, pressure, displacement, vibration, flow dynamics and liquid level.

The external perturbation could also be a thermal measurand. Temperature variation along the fiber causes a change in the effective reflective index due to the thermo-optic coefficient effects of the fiber. Furthermore, thermal expansion produces a change in the gratings periodicity making it possible to measure temperature. The thermal and axial strain response characteristics can be described in the following well known equation of FBG sensing technique [106]

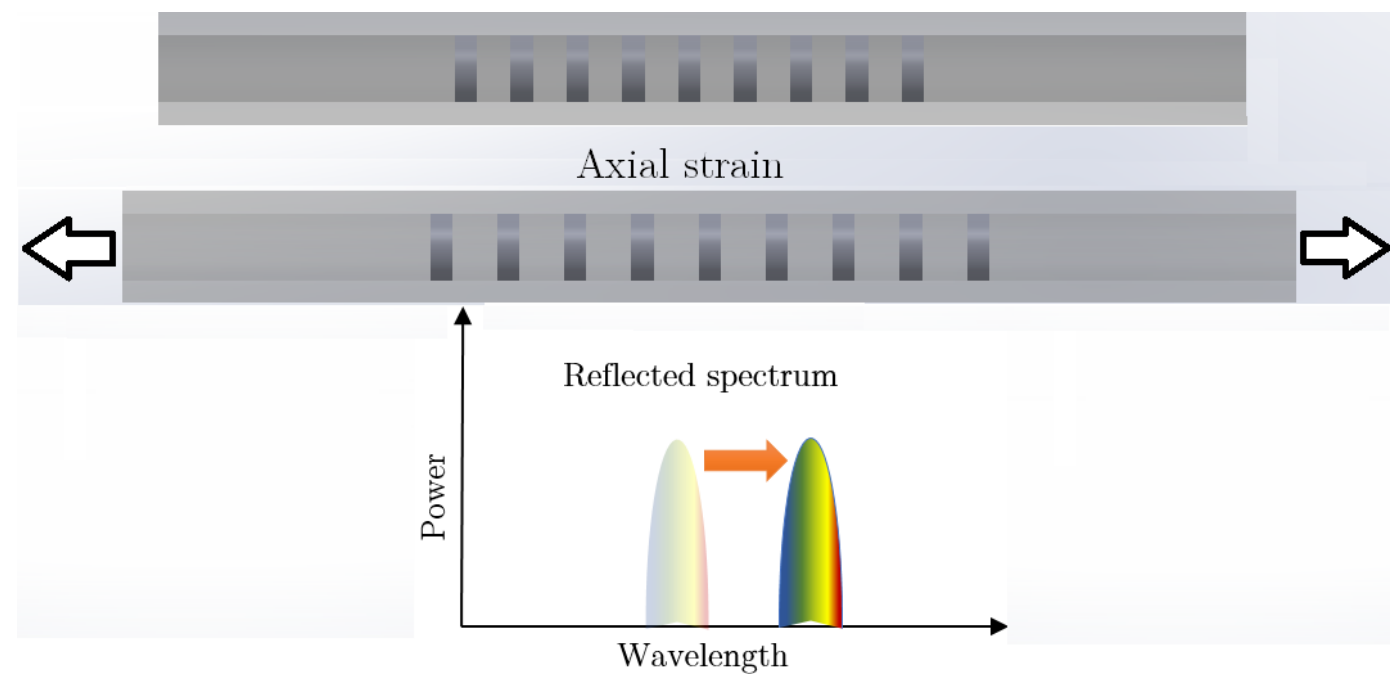

Figure 2.13: FBG sensing element and the reflected wavelength variation under uniaxial strain.

$$
\Delta \lambda_{B}=\lambda_{B}\left(\alpha_{f}+\xi\right) \Delta T+\lambda_{B}\left(1-P_{e}\right) \Delta \varepsilon
$$

where $\Delta \lambda_{B}$ is the wavelength shift of the reflected spectrum from the Bragg gratings 
generated due to the applied external perturbations such as strain, displacement, pressure, force, vibration. $\alpha_{f}, \Delta \varepsilon$ represent the thermal-expansion coefficient and the change in applied axial strain, respectively. $\xi$ and $P_{e}$ represent the thermo-optic and elesto-optic coefficients, respectively. $\Delta T$ is the temperature variations along the fiber. The coefficient of the thermo-optic is characterized as

$$
P_{e}=\frac{1}{2} n_{e f f}^{2}\left(p_{12}-\nu\left(p_{11}-p_{12}\right)\right)
$$

where $p_{12}$ and $p_{11}$ represent Pockel's coefficients of elesto-optic (strain-optic) tensor, and $\nu$ is Poisson's ratio of fiber material. The typical values of an optical fiber made of bulk silica are $p_{11}=0.121, p_{12}=0.27, n_{\text {eff }}=1.448$ and $\nu=0.16-0.17$ [107].

Equation (6) reveals that the reflected Bragg wavelength becomes shifted when a physical elongation is produced due to either a variation in the applied axial strain or in temperature. Longitudinal strain detection equation at constant temperature $(\Delta T=0)$ can be described as follows:

$$
\Delta \varepsilon=\frac{\Delta \lambda_{B}}{\lambda_{B}\left(1-P_{e}\right)}
$$

When only strain detection is required, additional FBG can be inscribed along the same fiber which can also be used as a temperature compensator. In this case, the additional FBG should not be susceptible to any longitudinal strain. The temperature change can be calculated when the strain is constant $(\Delta \varepsilon=0)$ as follows:

$$
\Delta T=\frac{\Delta \lambda_{B}}{\lambda_{B}\left(\alpha_{f}+\xi\right)}
$$

where, 


$$
\begin{aligned}
& \alpha_{f}=\left(1 / \Lambda_{B}\right)\left(\partial \Lambda_{B} / \partial T\right) \\
& \xi=\left(1 / n_{e f f}\right)\left(\partial n_{e f f} / \partial T\right)
\end{aligned}
$$

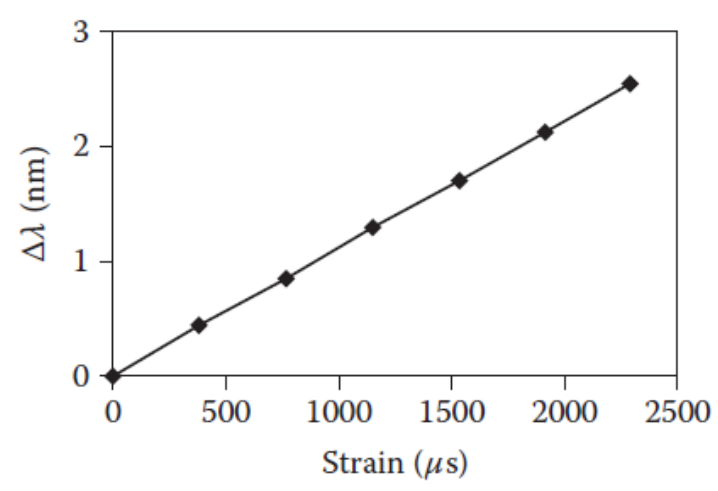

(a)

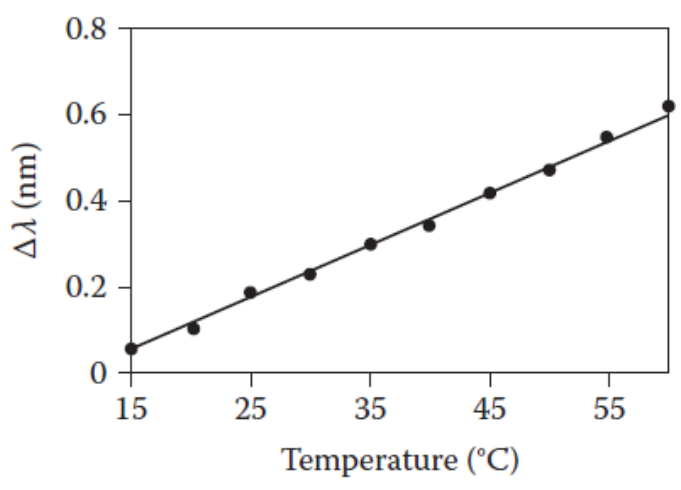

(b)

Figure 2.14: Strain and temperature sensitivities of an FBG-based sensor [15].

FBG based sensors exhibit a highly linear relationship between the applied axial strain and the reflected Bragg wavelength which is however, limited to the elastic deformation of the optical fiber material. Moreover, the FBG sensitivity to the applied strain relies on the photo-elasticity and Young's modulus of the fiber material used in the FBG fabrication and the cross-sectional area of the inscribed fiber Bragg gratings [108].

However, the FBG based sensors exhibit considerably less sensitivity to temperature change compared to the applied longitudinal strain, as depicted in Figure 2.14 [15]. The typical temperature sensitivity for the reflected Bragg wavelength is between 0.0068 $\mathrm{nm} /{ }^{\circ} \mathrm{C}$ and $0.013 \mathrm{~nm} /{ }^{\circ} \mathrm{C}$. The corresponding Bragg wavelength range is from $830 \mathrm{~nm}$ to $1550 \mathrm{~nm}$. The strain sensitivity starts form $0.00064 \mathrm{~nm} / \mu \varepsilon$ to $0.0012 \mathrm{~nm} / \mu \varepsilon$ for the same Bragg wavelength range [109]. 


\subsubsection{FBG-Based Sensors in Biomechanical and Gait Measurements}

FBG sensors have been among the dominating fiber-optic sensing technologies for strain, pressure and force sensing [108]. In biomechanical applications, introducing several advantages such as lightweight, small size, high accuracy, high longitudinal strain resolution, no sensitivity to electromagnetic interference, and negligible hysteresis effects would significantly enhance the performance of modern human assistive devices which have become a crucial part in the modern healthcare industry. The FBG-based sensors include all of the listed superior advantages [110, 111], which make them a strong potential solution to address the challenges associated with developing high-tech devices for rehabilitation and for biomechanical applications. Due to their high capabilities of sensing, FBG sensors have been used in ground reaction force measurements and gait analysis [112], body kinematics [113], prosthetic socks, smart medical beds [114] and surgical robots [115].

Monitoring ground reaction forces is essential to help maintain balance and to help prevent falls, avoiding their associated injuries [116] as well as diagnosing foot ulceration diseases [76, 117]; this is particularly beneficial for the elderly and for people with abnormal gait and impaired balance. The primary experimental technology for ground reaction force measurement is the force plate, but it is restricted to a laboratory setting and requires adjustments to the patient's walk during experiments in order to collect data. On the other hand, wearable pressure shoe sensors are desirable for this practical application, if they are properly designed. FBG-based sensors are a unique solution as they have many advantages over the conventional electric sensors. Several sensors based on FBG techniques have been developed for plantar shear and 
normal force measurements. An array of FBG pressure sensing units were embedded in carbon-composed material and integrated in shoe 's insole [118, 119]. The fiber consists of five Bragg gratings: two under the heel and three underneath the forefoot. Each sensor has been tested in only static loading conditions, by standing on the FBG arrays.

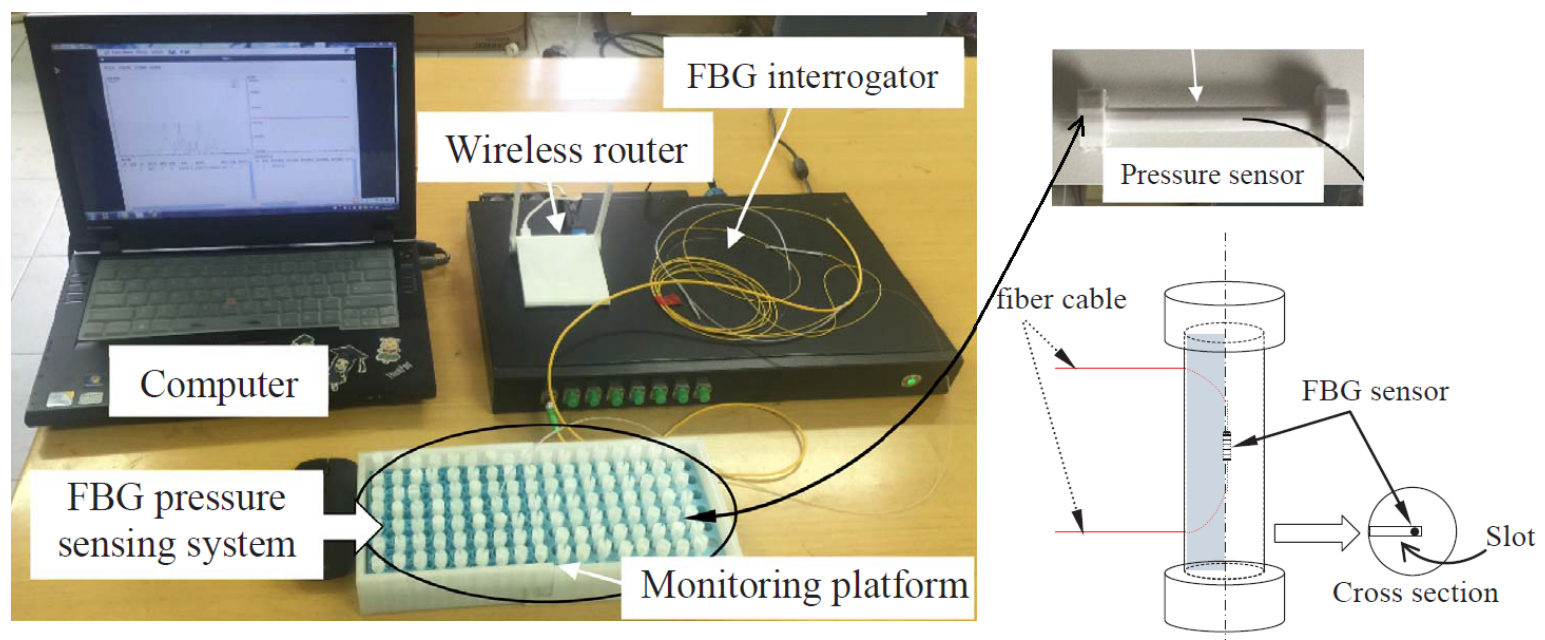

Figure 2.15: FBG-based sensor plantar normal force measurements. The FBG is integrated inside a 3D printed cylindrical bar through a slot made at the center of each sensing element [16].

A plantar pressure sensor platform was reported in [16]. The fiber employing FBG sensors was passed through a hole made in a cylindrical element which was 3D-printed in PLA (Polylactic Acid) with a diameter and height of $4 \mathrm{~mm}$ and $30 \mathrm{~mm}$, respectively. The dimensions of the sensor was considerably large which would not be possible to integrate into a shoe. In addition, the sensor was rigid and was limited to detect only one force component: vertical ground reaction force. The prototype is depicted in Figure 2.15. In an effort to increase sensor flexibility, six Bragg gratings were inscribed along an optical fiber and were embedded inside a silicon rubber as shown in Figure 2.16 [17]. However, only a static performance was demonstrated and only the normal (vertical) ground reaction force was measured but no shear forces were measured. Furthermore, temperature effects were not addressed in the aforementioned 
sensors.

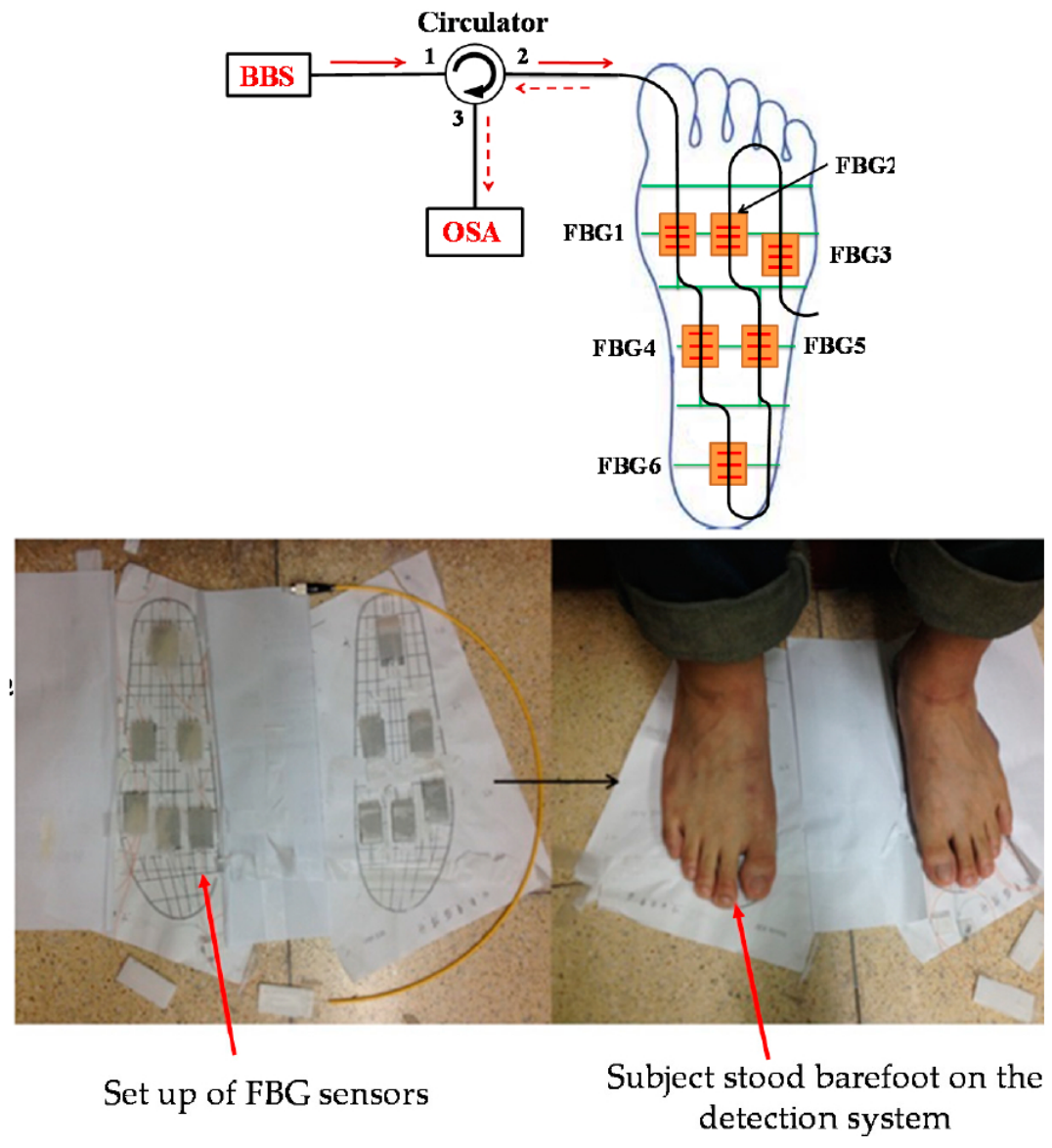

Figure 2.16: A flexible insole force sensor composed of six FBG sensing elements for distributive plantar normal pressure measurements [17].

FBG-based sensors have also been used for plantar shear force and strain measurements. In [18], the fiber with Bragg gratings was affixed between two rigid plates and tilted with a small angle. One end of the fiber is fixed to the bottom plate and the other end is attached to the top plate. The plates are sandwiched between two rigid beams made of the same material. The applied longitudinal force on the upper plate was converted to a corresponding axial strain along the fixed fiber, as depicted in Figure 2.17. However, the sensor was too rigid with a relatively high thickness of $30 \mathrm{~mm}$. 


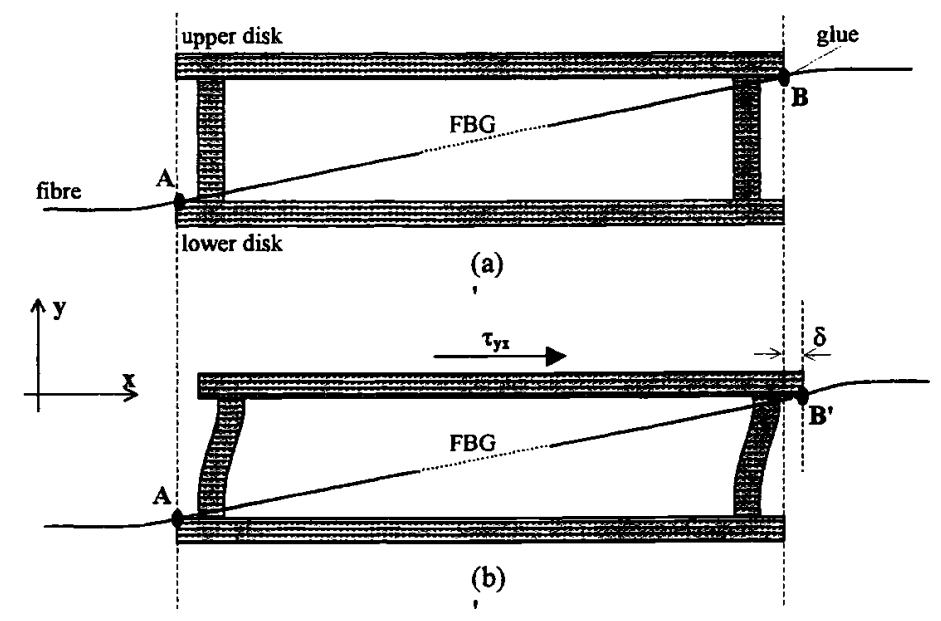

Figure 2.17: A rigid in-shoe shear force sensor affixed between to plates [18].

Improvements have been made to sensors of similar working principles [120]. The fiber was embedded into silicon rubber, with the top and the bottom layers made of carbon-composite material, as shown in Figure 2.18. The sensor in this configuration is more flexible with a thickness reduced to $1.8 \mathrm{~mm}$.
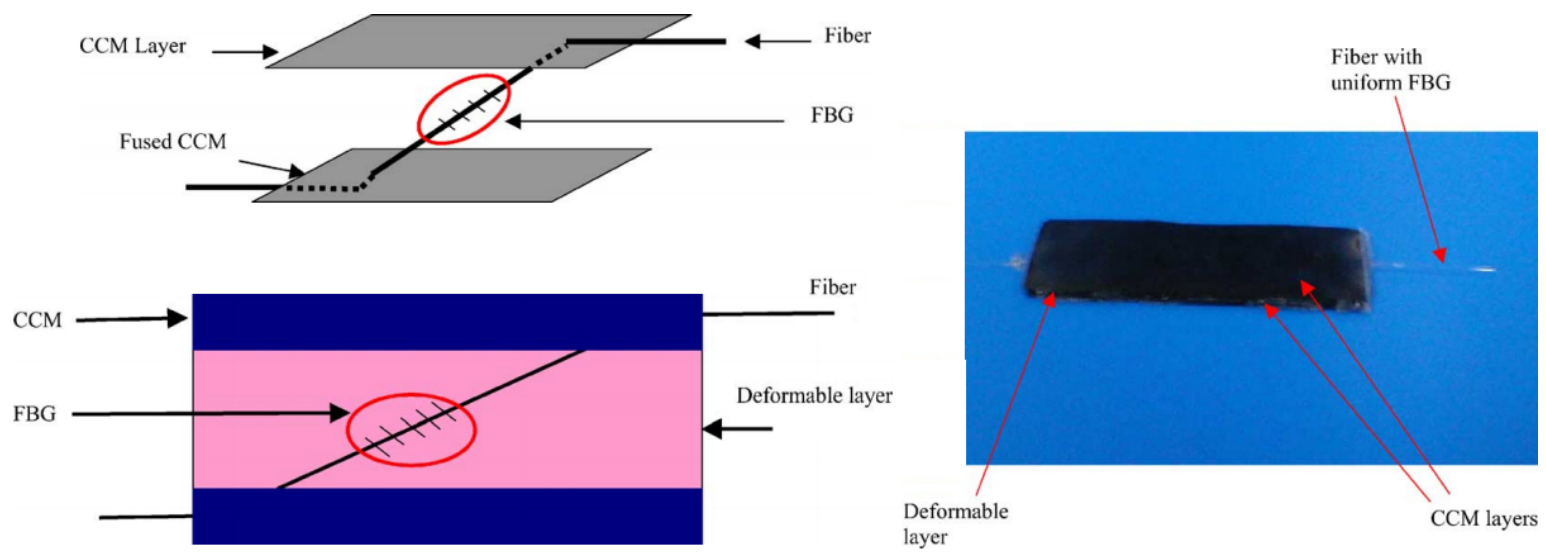

Figure 2.18: FBG-based sensing element for shear force measurements [19, 20].

Although numerical and analytical models were proposed in [121] which were relatively close to the experimental results, the sensor was only able to measure the shear strain in a single direction when the applied axial strain is positive. Therefore, 
the prototype cannot be used for gait measurements since the direction of shear force and strain underneath the foot changes during a walk or a run.

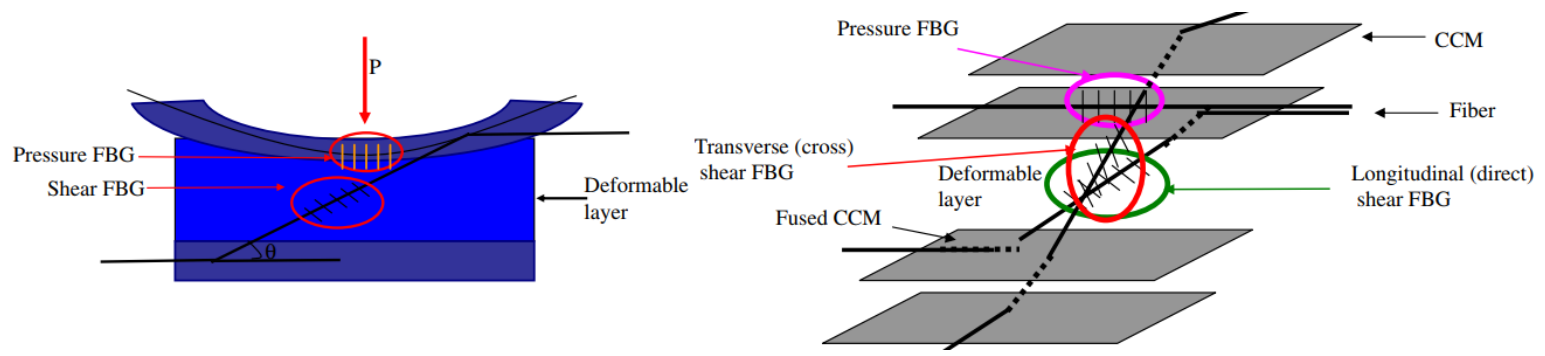

Figure 2.19: FBG-based sensing element for normal and shear forces measurements[20].

In an attempt to increase the sensing capabilities, an optical fiber with one Bragg grating was added in order to capture the applied normal force [20] in addition to the shear force. The fiber was horizontally embedded into the carbon composite of the top layer. When a vertical force is applied on the upper surface of the prototype, it creates a bend in the top layer; and therefore causes a wavelength shift proportional to the magnitude of the applied normal force. Moreover, another fiber was added to detect the other shear force component $(F y)$ that the previous version of the sensor could not capture, as it can be seen in Figure 2.19. The fiber was also embedded in the same medium layer of the silicon rubber but along the y-axis. Despite the possibility of measuring the force along the three normal axes with minimal crosstalk effects, the sensor was incapable of detecting the negative values of the applied shear forces.

Considerable improvements in the sensor performance have been reported in [21]. The shear and normal force fibers were not directly embedded in the silicon rubber. Vaseline was applied on the fibers' surface to avoid cohesion with the silicon during the curing process. In addition, two gaskets were adhered in each fiber and were affixed in both sides of the elastic middle layer which helped to stretch the fiber. Figure 2.20 shows a comparison between two models using the qualitative results of finite element 
analysis: one with the fiber as being embedded directly into the elastic material (a), and the other using gaskets (b). By doing so, the applied normal and shear forces were uniformally distributed along the fiber and the sensitivity of the prototype was also improved. However, this design could damage the sensor if the silicon deflection generated by the applied force exceeds the elastic deformation limit of the used fiber.

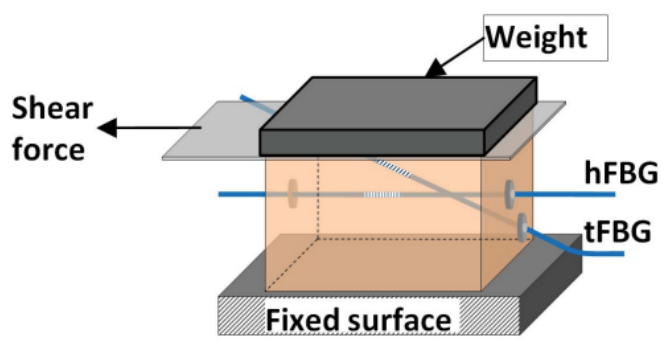

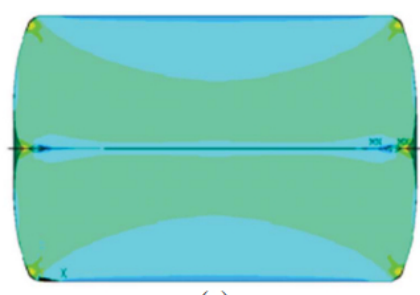

(a)

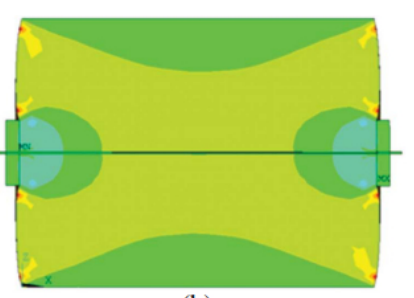

(b)

Figure 2.20: A flexible FBG-based force sensor for normal force and one component of shear force detection [21].

All of the above sensors were tested only in static loading conditions as one single sensing unit. No critical discussions were presented on how these sensors can be integrated in a shoe for practical use. In ground reaction force measurements and other biomechanical applications, the sensor's dynamic performance is essential but is not demonstrated or discussed.

In a recent work [22, 122-124], an instrumented insole sensor for distributive vertical ground reaction force has been developed and tested. Six FBG sensing elements were integrated into a shoe insole made of cork with $10 \mathrm{~mm}$ thickness as shown in Figure 2.21. Each FBG was embedded into an epoxy resin (rubber) for protection. Although the cork was chosen to reduce the temperature effects, additional FBG sensors were incorporated as a temperature compensator. While the prototype was dynamically tested for walking there was no standard reference to compare with or showing a regulated dynamic performance of the proposed sensor to match. Furthermore, the 
sensor was not capable of detecting shear forces in the $\mathrm{x}$ - and $\mathrm{y}$-direction, which are important force components in gait measurements.
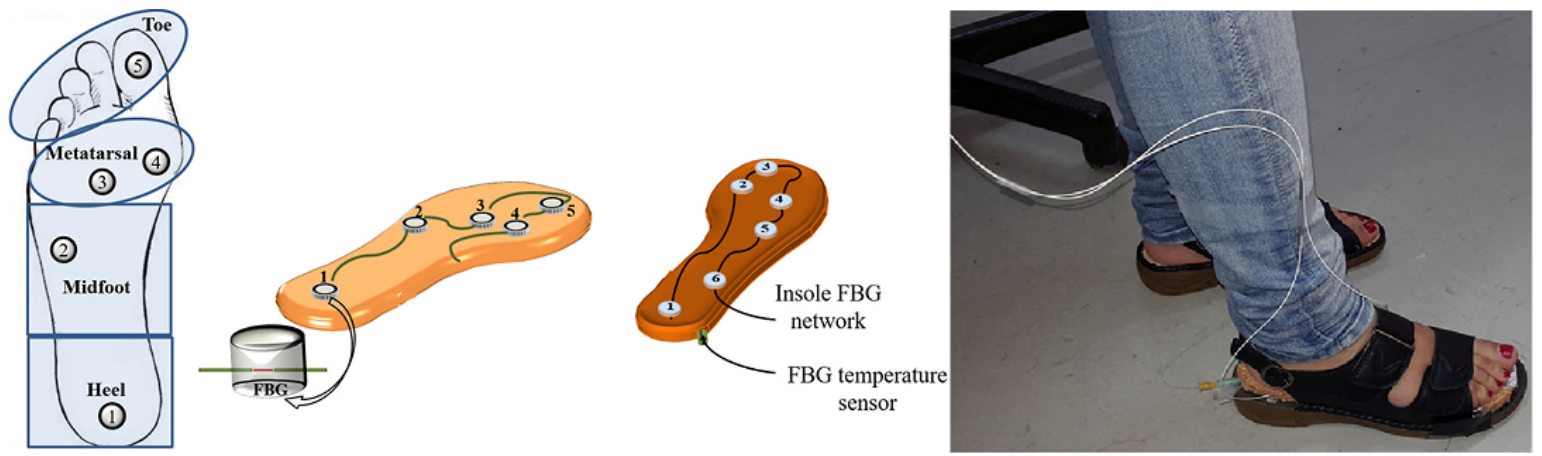

Figure 2.21: Instrumented insole sensor with six FBG sensing elements for vertical ground reaction force detection [22]. 


\title{
Chapter 3
}

\section{A Compliant 3-Axis Fiber-Optic Force Sensor for Biomechanical}

\section{Measurement}

\begin{abstract}
This chapter presents the development of a flexible, multi-axis, intensity modulated-based fiber-optic force sensor for concurrently measuring normal and shear forces. The proposed sensor was prototyped to measure the three force components by monitoring the variation of the light intensity induced by a deformation as a result of the applied force. One end of the sensor incorporates three orthogonal reflective planes. The other end brings three pairs of fibers; one fiber connected to an LED, and the other to a light-to-voltage converter LTV in each pair. Upon the application of the force, the distance between the planes and the fiber tips changes, thus, the LTV voltage changes,
\end{abstract}


enabling the simultaneous measurement of forces along three normal axes utilizing only one set of force measurement unit. The fabricated sensor was tested in both static and dynamic loading conditions as the experimental results have confirmed that the prototype has the capability to accurately measure the normal and shear forces in real time ranging from 0 to $1000 \mathrm{~N}$ and 0 to $140 \mathrm{~N}$ along the $\mathrm{z}, \mathrm{x}$ and $\mathrm{y}$ axes respectively. The feasible applications of the sensor are ground reaction force measurements (GRF) and robot-human collision detection. Sensor performance was evaluated for the cross-talk effects which were found to be less than 5\%. A nonlinear Hammerstein-Winer model is proposed to characterize the linear and nonlinear behavior of the sensor. The optimized results have shown a reduction of over $40 \%$ in the root mean-square errors (RMSE) in comparison to the linear estimation models.

\subsection{Introduction}

The rapid population growth of the elderly has increased the demand for effective healthcare systems. Developing human assistive technologies has become an essential part in the healthcare industry. In the meantime, recent advanced sensors and modern electronic technologies have made such development possible. It has been estimated that about a third of seniors aged 65 years or older fall down every year [125, 126]. Fall prediction for elderly and people with gait instability is crucial in preventing fall-related injuries [127-129]. Reliable measurement of normal and shear forces on a foot is imperative in gait analysis to help maintain balance and prevent falls [130].

Plantar ulceration is another disease that requires a precise assessment of plantar shear and normal forces [131, 132]. Studies have revealed that over three-quarters of amputation cases are attributed to foot ulcers [133, 134]. 
Remarkable efforts in literature have been dedicated to the development of distributive plantar shear and normal force measurement systems based on various detection principles. Force-sensitive resistors (FSRs) [135], capacitive and inductive based transducers [136, 137], piezoresistive [138] and piezoelectric-based plantar pressure sensors [139] have been proposed and prototyped. However, resistive materials are highly affected by temperature, structurally fragile, and are prone to damage. In addition, a temperature compensator is necessary for each individual sensing element [140].

All the abovementioned sensors are microelectromechanical systems technology, making them vulnerable to electromagnetic fields and external environmental perturbations. Moreover, These sensors were only capable of measuring normal force along the $\mathrm{z}$-axis but not shear forces in the $\mathrm{x}$ and $\mathrm{y}$ directions. Meanwhile, fiber-optic sensors are comparatively better candidates due to their immunity to electromagnetic interference and wide measurement range.

A fiber-optic sensor based on the bending-loss technique was demonstrated in [7]. The sensor was composed of two fiber meshes separated by a flexible pad to assess the normal and shear pressure based on light attenuation due to the material deformation. In [88, 141-143], the sensors were structurally improved by embedding the fibers into elastic material. However, the prototypes entailed too many fibers connected to detectors and light sources making it too bulky to integrate into a shoe. Furthermore, it was unfeasible to calibrate the sensor using the conventional calibration methods since the number of output signals exceeded the number of fibers (channels). A single fiber mesh contained eight fibers fixed on an elastic sheet creating several sensing points for the distributive force measurements [144]. The sensor showed a limited force measurement range of $2.5 \mathrm{~N}$ and can only measure the force applied along the z-axis. 
Optoelectronic transducers that incorporate only light sources and detectors without any fibers are also used for normal and shear force measurements. An insole pressure sensor was reported in [95]. A set of LEDs and photodetectors were integrated inside a flexible insole. Each LED faced its respective photodetector. When a physical deformation occurred due to the applied force, a small element in the insole prevents the light transmission from LEDs to photodetectors, allowing the normal pressure measurement based on the light variation. In [145] the LED and LTV pairs were used to measure the force along the three axes. Nevertheless, the lack of the flexibility made the prototype inconvenient in biomechanical applications.

Wavelength-modulation-based sensors such as fiber Bragg Gratings (FBGs) are another desirable option due to their high sensitivity, small size, and multiplexing sensing capabilities. The most recent work presented the feasibility of developing flexible sensors where the fibers were embedded into a silicon rubber [17, 22, 146, 147]. However, the sensors were capable of measuring only the normal plantar pressure but not shear, and were sensitive to temperature and very expensive to interrogate.

Compliant sensors have also been used in human-robot interactions. An optoelectronic force measurement system incorporated a matrix of LEDs and photodetectors connected on a printed-circuit-board (PCB) was reported in [148]. The matrix of the sensing elements was shielded by an elastic piece made of silicon rubber which had the shape of a hemisphere. The variation of the light intensity generated by a physical deflection was proportional to the applied force. The sensor was able to measure only the force along a single axis. In an effort to enhance the functionality of the sensor [149-151], the pressure sensing elements were distributed in a certain configuration to detect forces along the three axes. 
A multi-axis force and torque sensor based on optoelectronics technique was proposed in [152]. The sensor consisted of three small printed-circuit-boards PCBs and three LEDs. Each LED was centrally located on a PCB, surrounded by four photodetectors and fixed in a small cube. The alteration in the light intensity induced by the position and orientation change of the mirrors was utilized to determine the applied force and torque. The sensor was able to measure torques of up to $5 \mathrm{Nm}$. Cross-talk between different sensor axes, as a crucial characteristic of multi-axis sensors, was however, not considered in this work.

In our previous work, a Compliant Limb Sensor (CLS) was developed as a position sensing technique to detect robot-human collisions [153]. The sensor was designed to realize the displacement generated when a robot-human contact occurs. In CLS, an arrangement of light to voltage (LTV) sensors were used to measure displacement and orientation. The sensor exhibited very high compliance and displacements, but it required empirical models and relatively large online computation.

To address the aforementioned limitations and deficiencies, we present in this chapter, the development of a low-cost, compliant 3-axis fiber-optic force sensor with an adjustable sensing range feasibility for the simultaneous measurement of the three force components: normal and shear forces along the $\mathrm{z}, \mathrm{x}$ and $\mathrm{y}$ axes. The sensor presents high accuracy, low noise, insignificant cross-talk effects and wide force measurement range up to $1000 \mathrm{~N}$. The introduced technique is based on light intensity modulation using a special sensing mechanism that makes it easy to simultaneously assess forces along the three axes. 


\subsection{Sensor Design and Fabrication}

Figure 3.1 demonstrates the design concepts of the proposed 3-axis fiber-optic force sensor based on light intensity modulation by reflectance to measure the shear and normal forces in real-time. The sensor consists of three reflective surfaces for each sensor situated at the center of a square rigid aluminum plate (bottom layer) with dimensions of $5.5 \mathrm{~cm} \times 5.5 \mathrm{~cm} \times 0.1 \mathrm{~cm}$. The reflective surfaces are instantaneously utilized to reflect light from the transmitting fibers to the receiving fibers in each axis. Three plastic models (holders) were 3D printed in Acrylonitrile Butadiene-Styrene (ABS) material, which is the most commonly used in $3 \mathrm{D}$ printing and is suitable for this application due to its durability and rigidity with slight elastic-like characteristics. These holders are fixed on another aluminum plate (top layer) to carry the light detectors (LTVs) and light sources (LEDs) as well as the transmitting and the receiving fibers. Using these holders the fibers can be properly fixed and mounted to the LTVs and the LEDs. The fiber is made of copolymer plastic with a total diameter of $750 \mu \mathrm{m}$ and numerical aperture (NA) of 0.5. White LED with a power rating of $120 \mathrm{~mW}$ was utilized to provide the necessary light power. The reflected light is received by high sensitivity and low power consuming light to voltage converter (model: TSL257) that consists of a photodiode with a trans-impedance amplifier.

The top layer of the sensor also encompasses four elements used to orient the transmitting and the receiving fibers to their respective reflective surfaces via passing each pair of the fibers through small holes made in these elements. The transmitting and receiving fibers of the z-axis utilize one extra element to facilitate the bending process during fibers' installation to appropriately orient the fibers to their allocated reflective surface ( the bend seen in Figure 3.3 is fixed within the sensing unit, which 
does not move or bend during the operation). Each reflective surface is located perpendicular to its pair of fibers where the reflective surface of the normal force direction (force applied along the z-axis) lies along the bottom layer of the sensor. The reflective surfaces of shear forces are fixed perpendicular to the $\mathrm{x}$ and $\mathrm{y}$ axes.

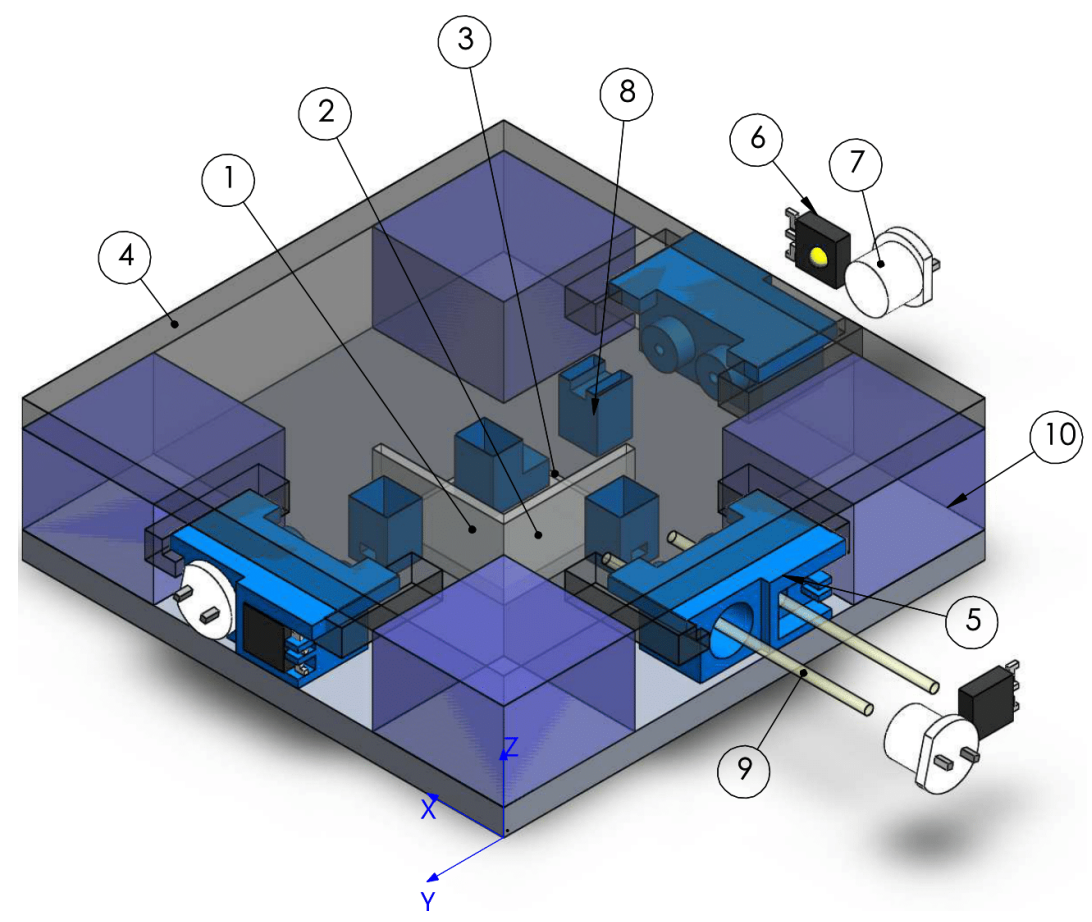

Figure 3.1: The fiber-optic sensing principles for the simultaneous measurement of normal and shear forces in the $\mathrm{z}, \mathrm{x}$ and $\mathrm{y}$ directions. (1), (7). The transmitting and receiving fibers of y-axis force sensor. (2). Reflective surfaces. (3), (4). Z-Axis transmitting and receiving fibers. (5), (6). The transmitting and receiving fibers of the $\mathrm{x}$-axis.

To increase the flexibility, a stretchable material such as rubber has been extensively utilized in developing a variety of flexible sensors [154-156]. In this work, four elastic cubes made from Mold Max 60 (a tin-cured silicon rubber of shore 60-A) features high temperature resistance capability. The dimensions of these cubes are $1.5 \mathrm{~cm} \times 1.5 \mathrm{~cm} \times 1 \mathrm{~cm}$ positioned at the four angles and sandwiched between the top and bottom layers, creating one sensing unit. 
The tin-cured silicon rubber was prepared by carefully mixing the Mold Max 60 with the curing agent at a specific weight ratio and cured at room temperature for 24 hours. As indicated in the manufacturer's guideline, the mixing ratio is 100:3 for Mold Max 60 silicon rubber and curing agent respectively. Since the ratio of the mixture can vary, the elasticity of the deformable material can be modified, thus, the measurement range of the sensor can be adjusted accordingly. In addition, the sensitivity of the sensor is influenced by the elasticity of silicon rubber.

The very top layer seen in the exploded view in the manuscript can be any material including metals such as steel, aluminum, or carbon fibre, depending on the application restrictions. These definitely provide options that can be decided for each application separately. Metallic layers can add extra strength and support the PLA layer as well. The four rubber blocks sandwiched between these layers have high viscoelastic properties compared to PLA, making the whole deflection occurs within the rubber. The main reason of using PLA-based material is due to its ease of manufacturing using 3D printing techniques. An important note here is that the real sensor with the PLA top which is more prone to deflections and consequential errors was used in the results presented, which still show good output accuracy despite this worst-case scenario. This confirms that most of the deflection comes from our rubber blocks.

The prototype is designed and manufactured as a single sensing unit that can be used in different biomechanical applications such as shoe insoles, collision detection, human-machine interaction, or other applications where rigidity of the sensor is not critical. 


\subsection{Sensing Mechanism and Working Principles}

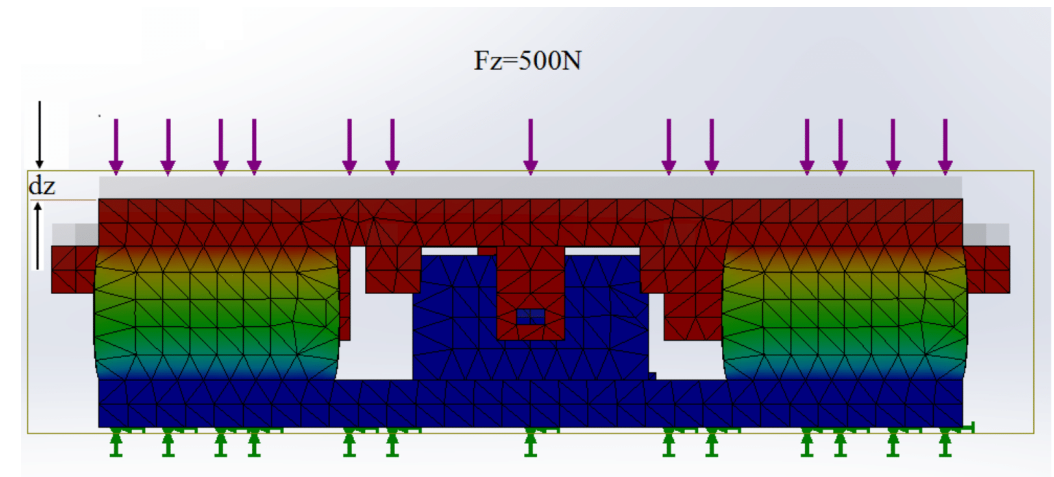

(a)

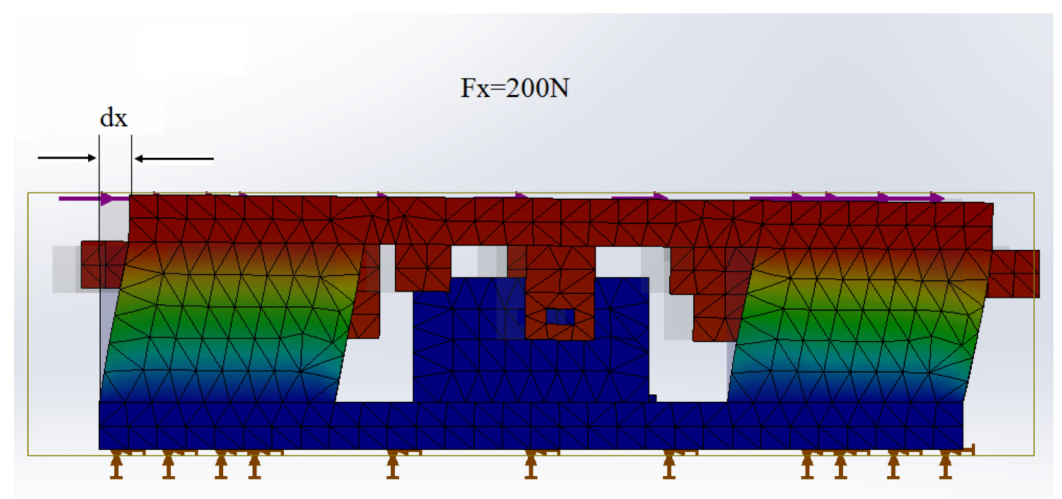

(b)

Figure 3.2: The sensing mechanism of the proposed sensor. (a) Illustrates the sensor under a vertical load of $500 \mathrm{~N}$. dz is the generated vertical displacement. (b) Shows the lateral displacement generated when $200 \mathrm{~N}$ of shear force along $\mathrm{x}$-axis is applied.

Figure 3.2 illustrates the sensing mechanism of the proposed sensor, where each couple of fibers is employed to assess the applied force in a certain direction. The fiber-optic operating principle of the sensor depends on the measurement of light power reflected by a fixed reflective target that typically relies on the target distance to the sensing tip. Each sensing tip is composed of one transmitting fiber that carries the 
light from the source to the reflecting target and one receiving fiber that accumulates the reflected light form the target as shown in Figure 3.3.

The receiving fibers are connected to photo-detectors to convert the light intensity to output voltage. The transmitting fibers are fed by light emitting diodes (LEDs). When the top layer of the sensor is excited by a pure normal force along the z-axis, the four cubes of the tin-cured silicon rubber are compressed and the displacement $\mathrm{dz}$ increases, representing the shrinking distance between the two layers of the sensor which leads to an increase in the light power. The light intensity in this instance, is a function of the generated vertical displacement. This is because the radius of the reflected light increases as result of the generated displacement as shown in Figure 3.4.

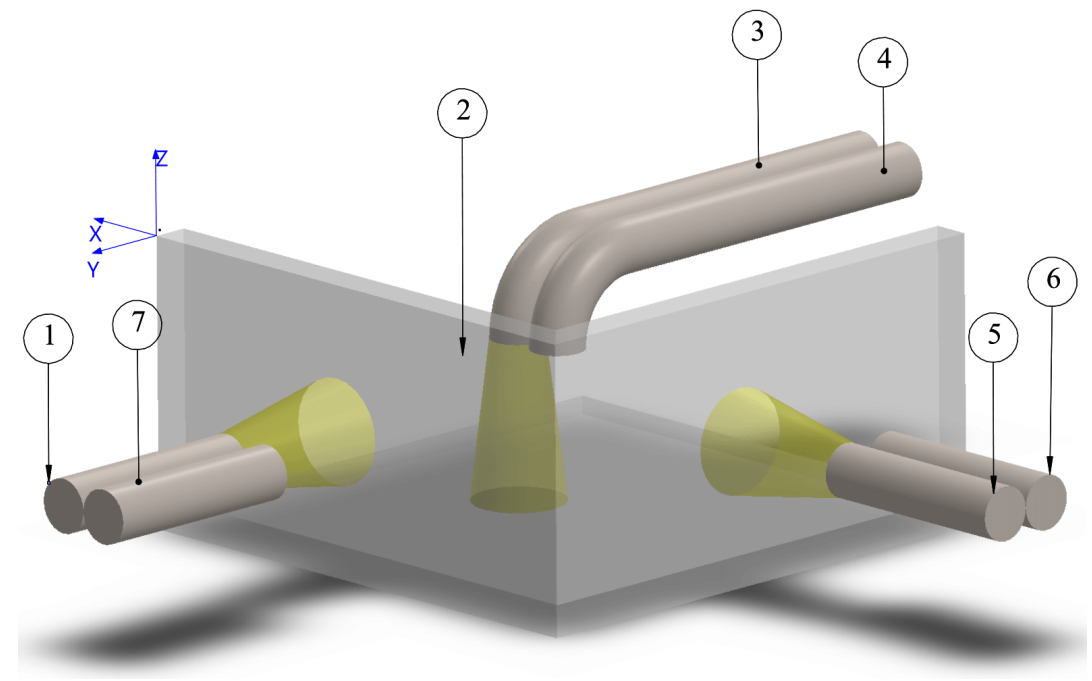

Figure 3.3: The fiber-optic sensing principles for the simultaneous measurement of normal and shear forces in the $\mathrm{z}, \mathrm{x}$ and $\mathrm{y}$ directions. (1), (7). The transmitting and receiving fibers of y-axis force sensor. (2). Reflective surfaces. (3), (4). Z-Axis transmitting and receiving fibers. (5), (6). The transmitting and receiving fibers of the $\mathrm{x}$-axis.

During this excitation, the fiber tips of the shear forces in the $\mathrm{x}$ and $\mathrm{y}$ directions stay parallel to their reflective targets. This means that the distance between the fiber 
tips (the transmitting and receiving fibers of the shear force ) and the targets remains constant under any value of the applied normal force. The intensity of the light does not change, therefore, the output voltage of these sensors will be constant and the force sensor is only sensitive to the applied normal force in the z direction. Similarly, when a pure shear force is applied along the x-axis, the lateral displacement increases and the fiber tips approach the reflecting target. In doing so, the distance decreases and the intensity of reflected light increases. Meanwhile, the fiber tips of the $\mathrm{z}$ and $\mathrm{y}$ axes stay parallel to their reflectors. The sensor in this case, is only sensitive to the $\mathrm{x}$ direction and the light intensity of the other axes does not change since the distance remains the same. The same principles apply to the y-axis of the shear force. Such transducer allows measuring the three forces independently with minimal crosstalk. In addition to the experimental results, the finite element analysis (FEA) was used in the design stage which provided results that matched the experimental results. The sensing system incorporates a complex nonlinear dynamic behaviour of the elastic material and the nonlinearity stems from light-deflection relationship. The combination of these two complex systems leads to a complex and an inaccurate analytical model since such solutions are based on several assumptions such isotropic elasticity, negligible deformation in cross beams etc. Therefore, the experimental results, the finite element analysis were enough for this work .

\subsection{Experimental Results and Calibration}

In order to acquire a precise relationship between the applied force and the intensity of the reflected light, two experimental setups were used to characterize the sensor in the $\mathrm{z}, \mathrm{x}$ and $\mathrm{y}$ directions. Several experimentations were carried out by varying the force in 


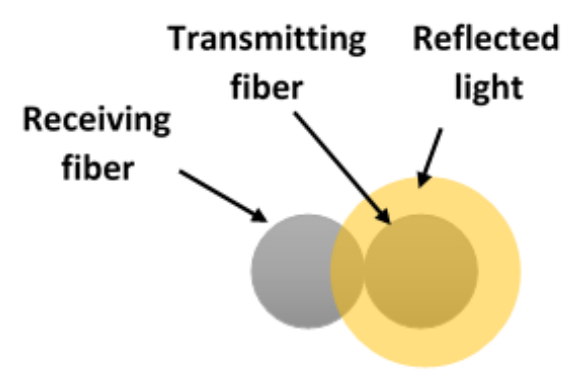

(a)

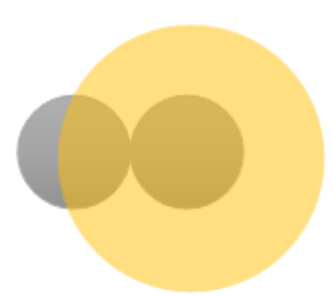

(b)

Figure 3.4: Sensing principles. (a) occurs when a small amount of force is applied. (b) occurs when the applied force increases.

the $\mathrm{x}, \mathrm{y}$ and $\mathrm{z}$ directions. Due to the lack of simultaneous three-axis force test systems, we designed and constructed a multi-axis force test device using 6-axis translation stage that has a resolution of $0.01 \mathrm{~mm}$ and a 6 -axis force and torque sensor (Model: F/T Delta SI-60-600, ATI Industrial Automation). The sensor features a measurement range of $660 \mathrm{~N}$ for the force along the $\mathrm{x}$ and $\mathrm{y}$ axes and $1980 \mathrm{~N}$ for the normal force with a resolution of $0.25 \mathrm{~N}$. The sensor is appropriate for this calibration since the fabricated sensor operates within that range. An aluminum platform composed of two plates is connected to each other by four cylindrical beams as shown in Figure 3.5 (a). The stage is fixed to the top plate and the F/T Delta SI-60-600 sensor is located at the center of the bottom plate. The proposed sensor is placed between the translation stage and the 6 -axis $\mathrm{F} / \mathrm{T}$ sensor. Forces in different directions can be applied by adjusting the stage in several positions. The data acquisition-system incorporates a CB-68LP National Instruments, 16-bit multi-function DAQ card, MATLAB Real Time Simulink software and a desktop computer.

The fabricated force test device has the ability to apply forces simultaneously in different directions compared to the Bionix Servo-hydraulic Test System (MTS) that applies the force only in one direction as depicted in Figure 3.5 (b). However, the 


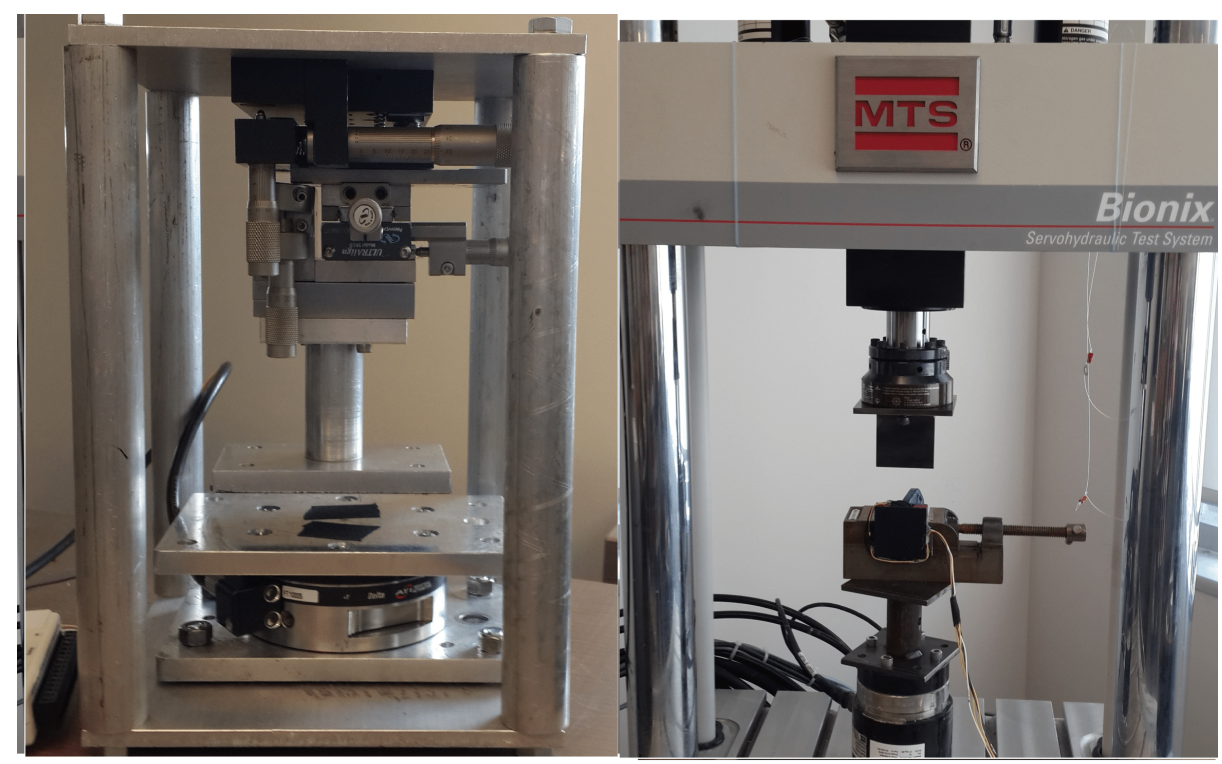

(a)

(b)

Figure 3.5: (a) The developed test device that is mainly composed of ATI 6-axis F/T sensor and 6-axis stage. (b) The MTS experimental setup was utilized to apply pure uniaxial force to evaluate the cross-talk effects.

MTS was more accurate in terms of linear motion during the uniaxial loading test. As a consequence, we used the MTS setup to properly investigate the cross-talk effects. The device has a precise linear-variable displacement-transducer with a resolution of $0.01 \mathrm{~mm}$ and $1 \mathrm{~N}$. We carried out the normal force test (compression) by placing the sensor between the MTS grips in the test area. Notice that the thickness of the top and bottom layers of the designed sensor was very small making it hard to apply shear forces using the MTS so that we replaced the old layers with 3D-printed ones with a thickness of $3 \mathrm{~mm}$. For the shear force test, the same setup was used by twisting the sensor in the $\mathrm{x}$ or $\mathrm{y}$ direction and applying different loads. To evaluate the cross-talk of the sensor, the force was applied individually along each axis using the MTS and the response of the other two axes was monitored. This assessment guaranteed that no undesired forces could disturb the uniaxial loading test when a force in a certain direction is applied. 
A preliminary characterization was done using a quadratic function for each axis ( $\left.F=a_{1} V^{2}+a_{2} V+a_{3}\right)$, where $F$ is the applied force, $V$ is the output voltage, and $a_{1}, a_{2}$ and $a_{3}$ are the coefficients. The force-voltage measurements and the fitted curves for static loading are shown in Figure 3.6. The RMSE values were found to be $7.71 \mathrm{~N}$ $\left((0.76 \%), 1.80 \mathrm{~N}(1.28 \%)\right.$ and $2.76 \mathrm{~N}(1.97 \%)$ for the $\mathrm{z}^{-}, \mathrm{x}-$ and $\mathrm{y}$-axis respectively.

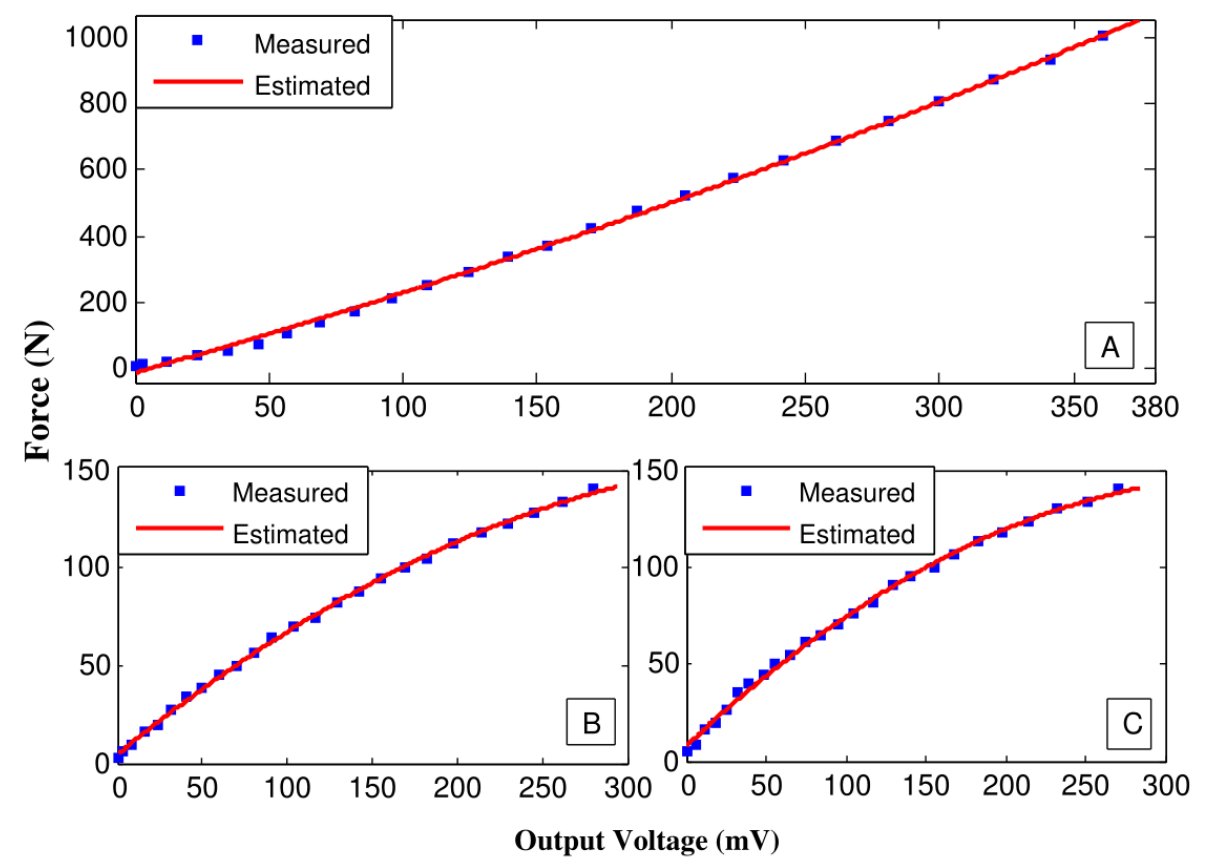

Figure 3.6: Quadratic fitting model of force to voltage curves. (A), (B) and (C) are the fitting models of the $\mathrm{z}-, \mathrm{x}-$ and $\mathrm{y}$-axis respectively.

\subsubsection{The Effect of Cross-talk on Sensor Performance}

Force signals acquired from a multi-axis force sensor other than the force being measured in the target direction are called cross-talk. These unwanted signals inevitably cause errors, affecting the performance of the force measurement. Cross-talk effects are commonly considered to be a problem in sensor development. It is very desirable for a sensor to produce the outputs directly proportional to individual inputs 
( $F x, F y$ and $F z$ : Forces applied along the $\mathrm{x}-, \mathrm{y}-$, and the $\mathrm{z}$-axis respectively) without cross-talk. As high as $28 \%$ of cross-talk was reported in [157]. In [158, 159] a mathematical compensation method was used which, however, produced cross-talk errors of $5 \%$ and $10.61 \%$ respectively. In the datasheet of the commercially-available multi-axis force sensors (KISTLER, Models: 9167A, 9168A, 9167AB and 9168AB) a cross-talk of $5 \%$ was presented. In this section, several experiments (static tests) were conducted and repeated several times to evaluate the cross-talk effects on the sensor under different magnitudes and directions of the applied force. Normal force ranging from 0-1 $\mathrm{kN}$ was applied to the proposed sensor, such that the four rubber cubes were under a compression. The response of the sensor in the three directions of $\mathrm{z}, \mathrm{x}$ and $\mathrm{y}$ is depicted in Figure 3.7 (a)-(c)

Although the experimental relationship between the light intensity and the displacement is nonlinear, Figure 3.7 (a) shows good linearity between the applied normal force and the light intensity. The linearity of the z-axis was achieved as a result of a nonlinearity cancellation phenomenon that occurred when the mechanical nonlinearity of the elastic material (between the applied normal force and the generated displacement) cancelled the nonlinear relationship between the light intensity and the displacement. The cancellation between these nonlinearities led to overall linear correlation that can be distinctly observed in Figure 3.7 (a). The level of cancellation can be controlled by adjusting the stiffness of the elastic material.

The results also showed that the sensor responded only to the applied normal forces which entirely agrees with our theoretical discussion of the working principles in the previous section. The maximum cross-talk errors in the $\mathrm{x}$ and $\mathrm{y}$ directions were $2.12 \mathrm{~N}$ (1.51\%) and $4.31 \mathrm{~N}(3.07 \%)$ receptively. A negligible sensitivity in both of the $\mathrm{x}$ and y axes to the applied normal force is presented in Figure 3.7 (a) 


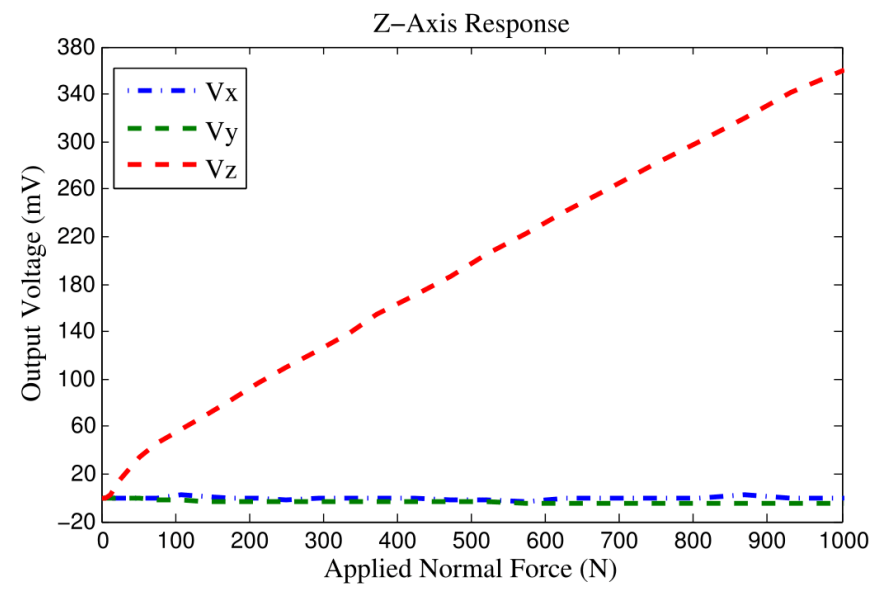

(a)

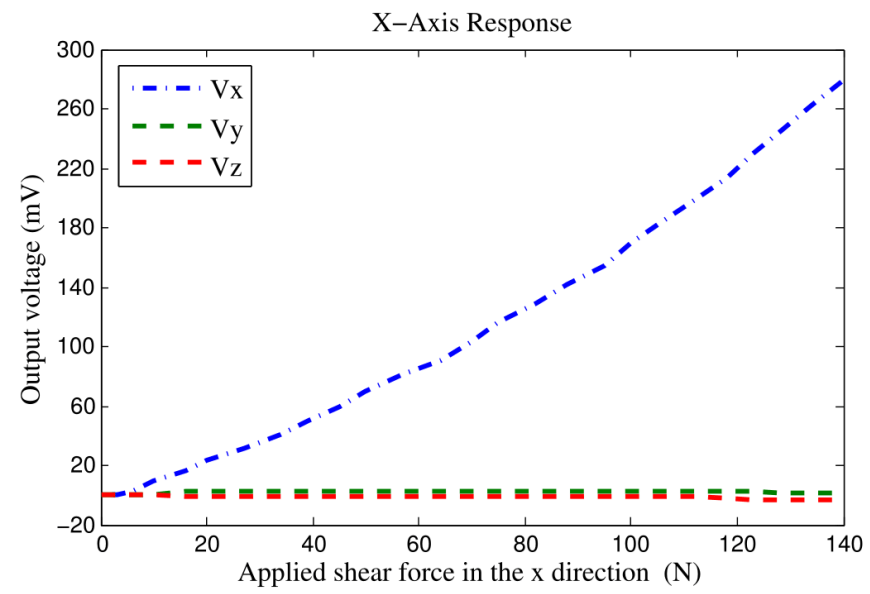

(b)

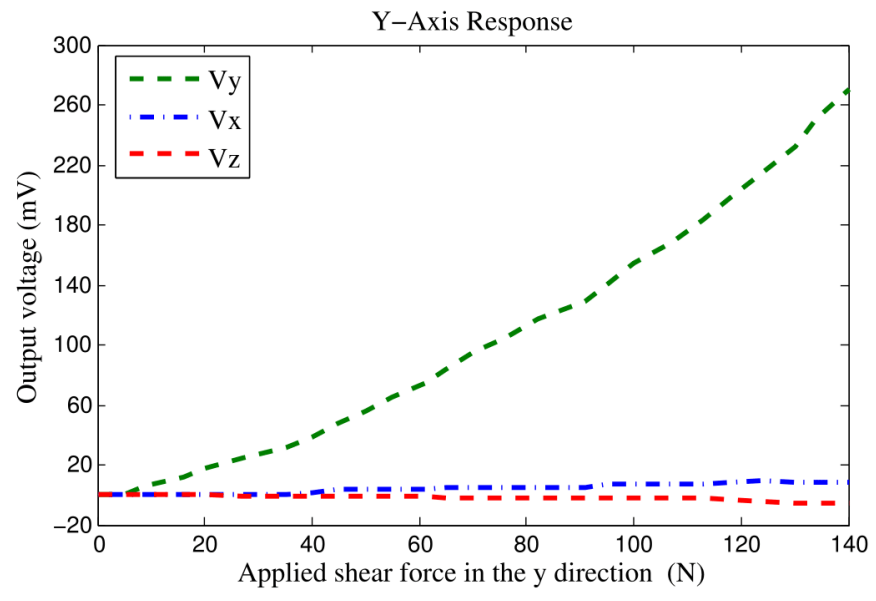

(c)

Figure 3.7: Sensor response to the applied external force along the three normal axes using the MTS. 
Table 3.1: A summary of the cross-talk errors along each axis

\begin{tabular}{|c|c|c|c|}
\hline \multicolumn{4}{|c|}{ X-Axis } \\
\hline$F_{Y}($ Max $)$ & $F_{Y}($ Max $) \%$ & $F_{Z}($ Max $)$ & $F_{Z}($ Max $) \%$ \\
\hline $2.60 \mathrm{~N}$ & $1.85 \%$ & $3.78 \mathrm{~N}$ & $0.37 \%$ \\
\hline \multicolumn{4}{|c|}{ Y-Axis } \\
\hline$F_{X}($ Max $)$ & $F_{X}($ Max $) \%$ & $F_{Z}($ Max $)$ & $F_{Z}($ Max $) \%$ \\
\hline $5.62 \mathrm{~N}$ & $4.01 \%$ & $13.79 \mathrm{~N}$ & $1.37 \%$ \\
\hline \multicolumn{4}{|c|}{ Z-Axis } \\
\hline$F_{X}($ Max $)$ & $F_{X}($ Max $) \%$ & $F_{Y}($ Max $)$ & $F_{Y}($ Max $) \%$ \\
\hline $2.12 \mathrm{~N}$ & $1.51 \%$ & $4.31 \mathrm{~N}$ & $3.07 \%$ \\
\hline
\end{tabular}

Figure 3.7 (b) illustrates the results obtained from a static shear force test in the $\mathrm{x}$ direction. In order to apply a pure shear force along the x-axis, a fixture was made to orient the sensor at the proper direction. A longitudinal shear force ranging from 0 to $140 \mathrm{~N}$ was applied. The figure does not show a linear correlation similar to that in the $\mathrm{z}$ direction between the applied shear and light intensity. This is due to the linear response of the elastic material to the applied shear force. Therefore, no nonlinearity cancellation was observed.

Figure 3.7 (b) also presents the greatest sensitivity to the direct shear force in the $\mathrm{x}$ direction, while the other two axes ( $\mathrm{y}$ and $\mathrm{z}$ ) revealed a negligible sensitivity with maximum cross-talk errors of $2.60 \mathrm{~N}(1.85 \%)$ in the $y$ direction and $3.78 \mathrm{~N}(0.37 \%)$ along the z-axis which also confirms the previously stated theoretical predictions. Similarly, the sensor was highly sensitive to the shear force in the y direction when a longitudinal force ranging from $0-140 \mathrm{~N}$ along the $\mathrm{y}$-axis was applied as shown in Figure 3.7 (c) However, maximum cross-talk errors of $13.79 \mathrm{~N}(1.37 \%)$ and $5.62 \mathrm{~N}$ (4.01\%) were found in the $\mathrm{z}$ and $\mathrm{x}$ directions respectively. These effects are all summarized in table 3.1. 


\subsubsection{Calibration Methodology}

Figure 3.8 shows the calibration setup of the proposed 3-axis force sensor that is placed between the ATI 6-axis F/T sensor and 6-axis manipulator. The force and torque sensor is utilized to measure the applied force (normal and shear) in the $\mathrm{z}, \mathrm{x}$ and $\mathrm{y}$ directions. The manipulator is used to apply forces along the three axes. The top layer of the fabricated sensor is connected to the manipulator via an aluminum bar fixed in the center of a square plate and the bottom layer is positioned on the ATI 6-axis $\mathrm{F} / \mathrm{T}$ sensor. When the stage moves along the z-axis, the sensor will be compressed and a

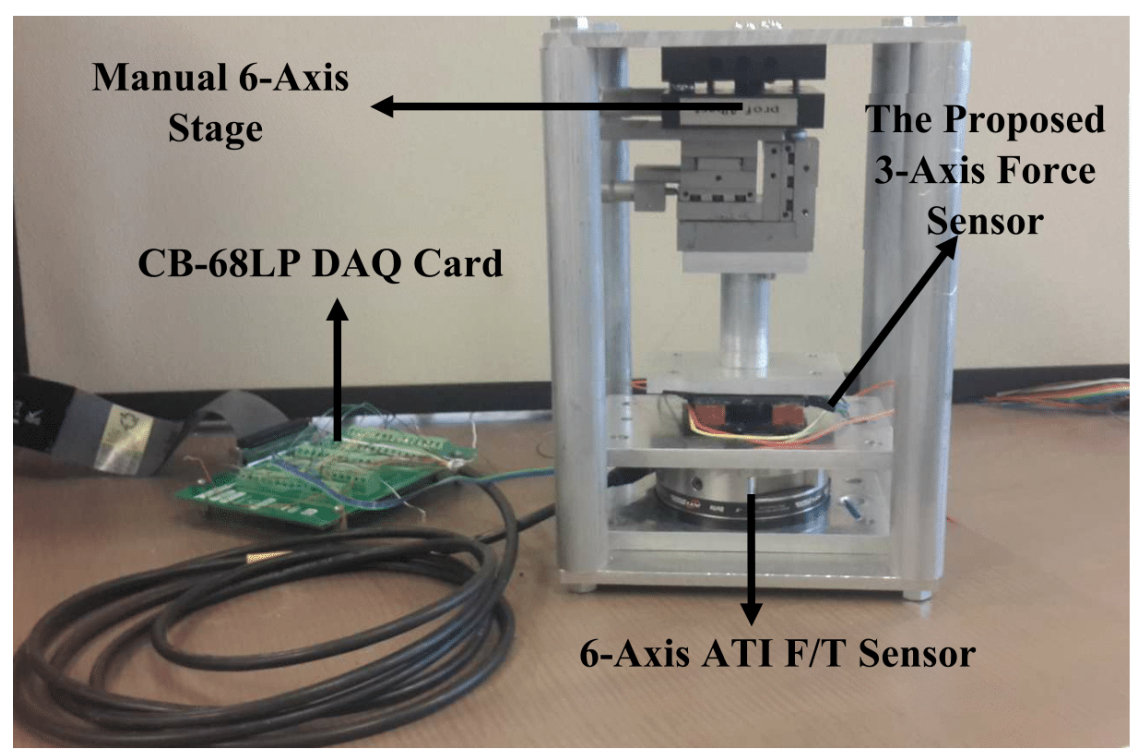

Figure 3.8: (a) The developed test device that is mainly composed of ATI 6-axis F/T sensor and 6-axis stage. (b) The MTS experimental setup was utilized to apply pure uniaxial force to evaluate the cross-talk effects.

uniform normal force is applied. The lateral movement of the stage in the $\mathrm{x}$ direction will shift the top layer of the sensor, generating a shear force along the $\mathrm{x}$-axis. In the same way, shear forces can be applied along the y-axis.

For the calibration, reference force data was collected by connecting the sensor 
and an ATI 6-axis $\mathrm{F} / \mathrm{T}$ sensor to the data acquisition card, which is connected to a computer using the RC68-68 ribbon cable. The data was acquired from both sensors at a sampling rate of $1 \mathrm{kHz}$. It was difficult to accurately understand the relationship between the sensor input and output using a conventional least-squares technique in the calibration process of the proposed sensor, due to nonlinearity.

Several models were applied using the system identification toolbox in MATLAB using roughly 5,000 samples of a database. For the x-axis, both a third-order Auto-regressive moving-average (ARMAX) estimation model and a second-order Output-Error (OE) model exhibited satisfactory results. The Root-Mean Square Error (RMSE) for each model was $7.32 \mathrm{~N}$ and $8.28 \mathrm{~N}$ with a fitting value of $85.88 \%$ and 84.04\% for the ARMAX and OE models respectively. A fifth-order transfer function estimation model with five poles and four zeros as presented in Equation 1 showed a better result with an RMSE of $6.54 \mathrm{~N}$ and a fitting value of $87.40 \%$. The results of the models as a comparison to the real data of the ATI 6-axis F/T sensor is plotted in Figure 3.9.

$$
G(z)=\frac{-197 z^{-1}+589.4 z^{-2}-588 z^{-3}-195 z^{-4}}{1-4.5 z^{-1}+5.5 z^{-2}-8.5 z^{-3}-4.4 z^{-4}-0.9 z^{-5}}
$$

The previously selected estimation models have been found to be powerful in characterizing the sensor. As a result, the three models were applied for each axis. With respect to the fitting value, the results of the $y$-axis were slightly more accurate than the $\mathrm{x}$-axis results. Using a fifth-order transfer function estimation model, a fitting value of $89.19 \%$ was obtained, as shown in Figure 3.10. The RMSE was 5.21 N. A second-order ARMAX and OE models were minimally less accurate than the transfer function model. The RMSE and the fitting values of both models were $6.24 \mathrm{~N}, 6.32 \mathrm{~N}$, 


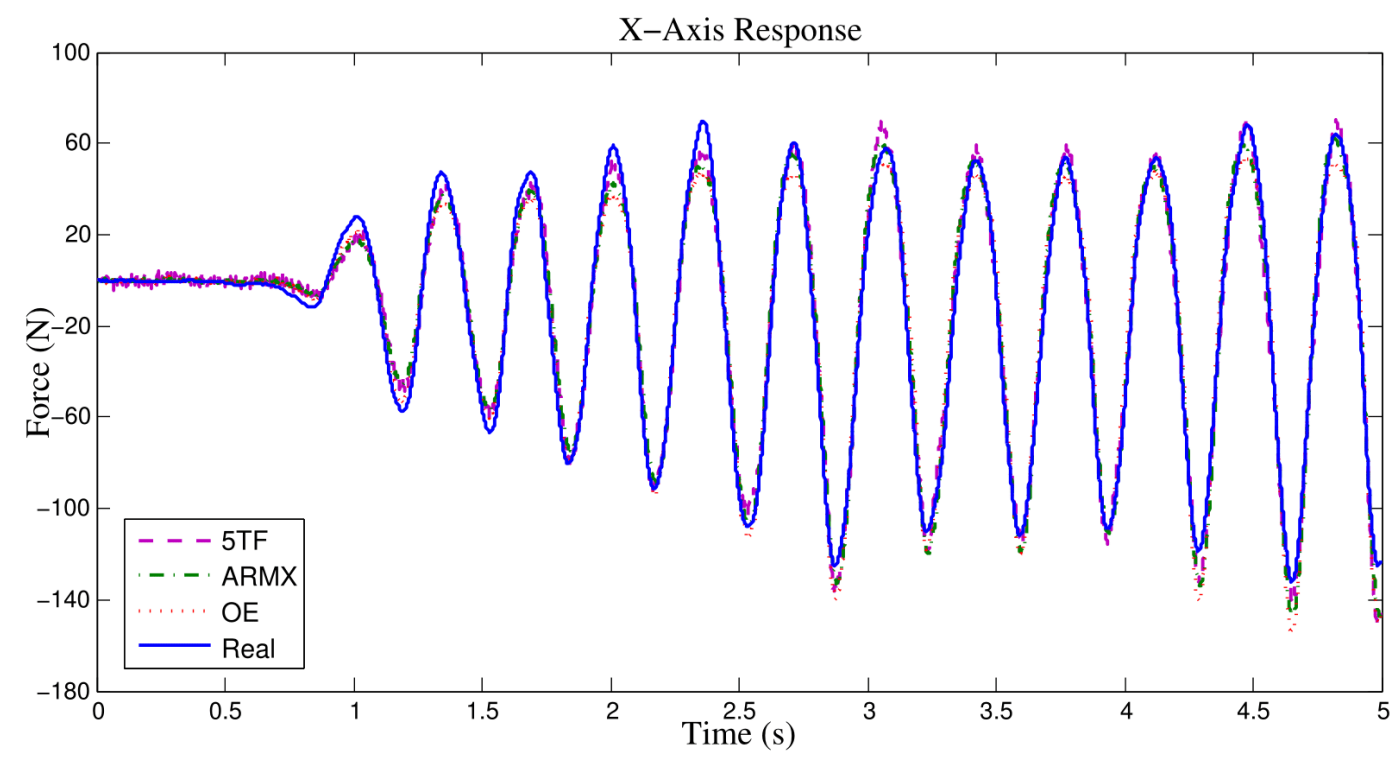

Figure 3.9: The experimental results of the $\mathrm{x}$-axis in a comparison to the acquired data from the ATI 6-axis F/T sensor. The plot shows a second-order Output-Error, a third-order Auto-regressive-moving-average (ARMAX) and a second-order transfer function model corresponding to the real data.

$87.01 \%$ and $86.86 \%$ for the ARMAX and OE respectively.

Although the z-axis presented the best results in terms of the fitting value which is $91.72 \%$ when an estimation model of fifth-order transfer function with five poles and four zero was used. The RMSE was found to be much higher: $20.27 \mathrm{~N}$ or $(2.63 \%)$ with a maximum error of $10.14 \%$. This is mainly because the dynamic test range was much more higher, ranging from $0 \mathrm{~N}$ to about $800 \mathrm{~N}$ compared with the other two axes. A second-order OE disclosed the same result as the last transfer function, whereas the RMSE and maximum error of a second-order ARMAX model were $23.91 \mathrm{~N}$ and $10.58 \%$ with a fitting value of $88.97 \%$. The dynamic response of the z-axis is illustrated in Figure 3.11. 


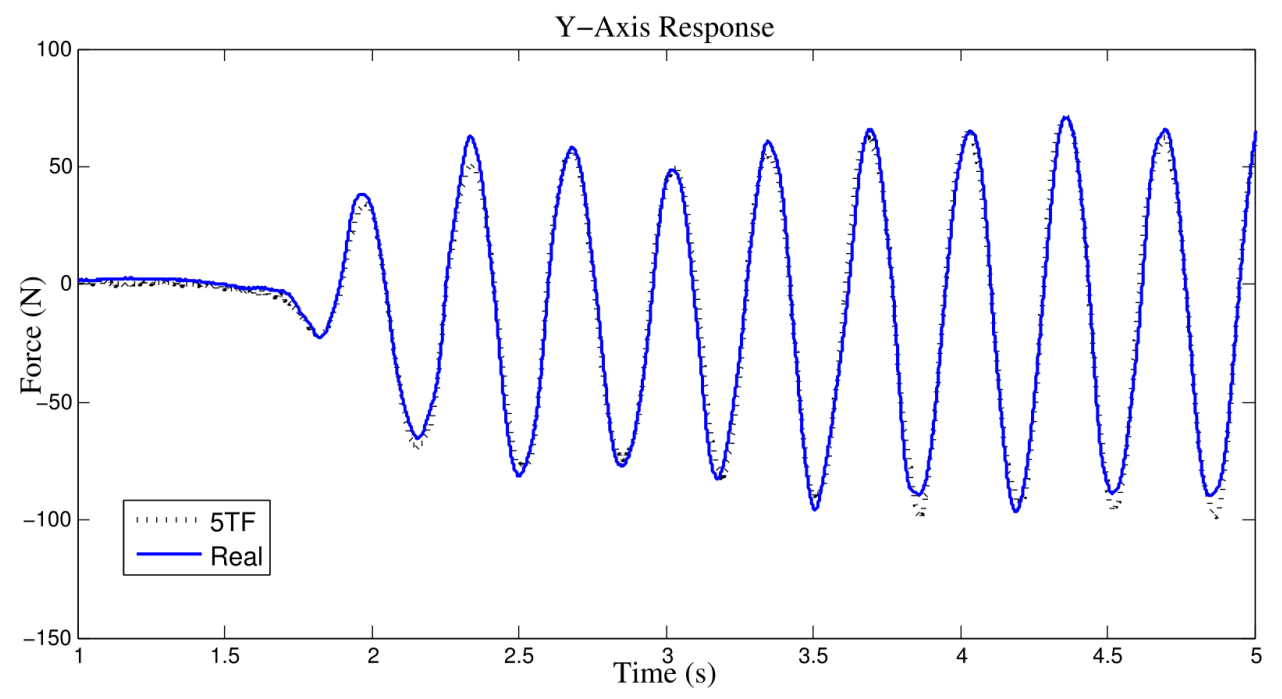

Figure 3.10: A comparison between the real data and the y-axis model. The graph demonstrates a second-order transfer function model compared to the real data.

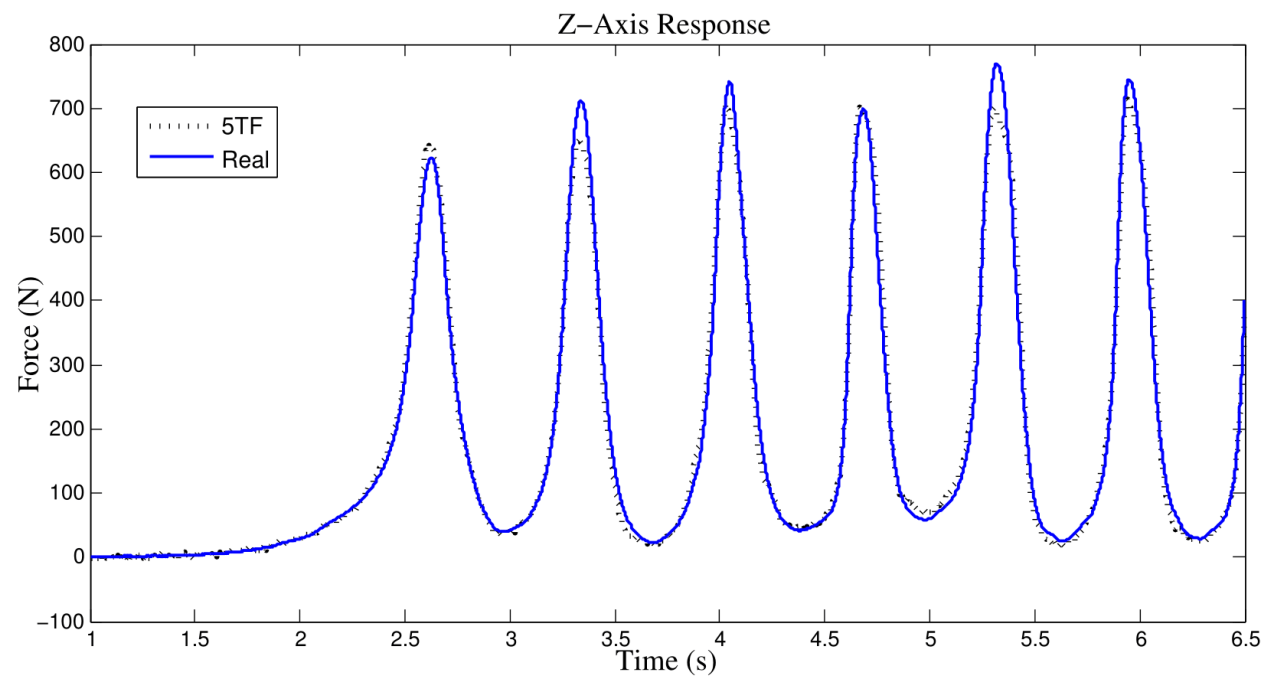

Figure 3.11: The similarity between the z-axis results using a second-order transfer function estimation model and the real data. 


\subsubsection{Nonlinear Hammerstein-Wiener Model}

Linear models exhibt a limited performance, mostly because of the nonlinear behavior of the real systems. Consequently, nonlinear system identification by means of nonlinear Hammerstein-Wiener (NLHW) estimation model was used. The NLHW is defined as a series connections of linear dynamic functions with a static nonlinear [160]. The interest in this model is expanding due to its low-cost computation process, simple to comprehend and straightforward to use in control systems [161]. The Hammerstein-Wiener model can characterize the linearity by pulse transfer, impulse response and state space estimation models. Meanwhile, the nonlinearity can be represented by piecewise linear, wavelets network, polynomial expressions, sigmoid network, look-up tables and fuzzy models [162-164]. Figure 3.12 demonstrates the schematic block-diagram of the Hammerstein-Wiener model. The results were enhanced significantly. The model showed improvements of up to $10 \%$ compared to the previous linear estimation models.

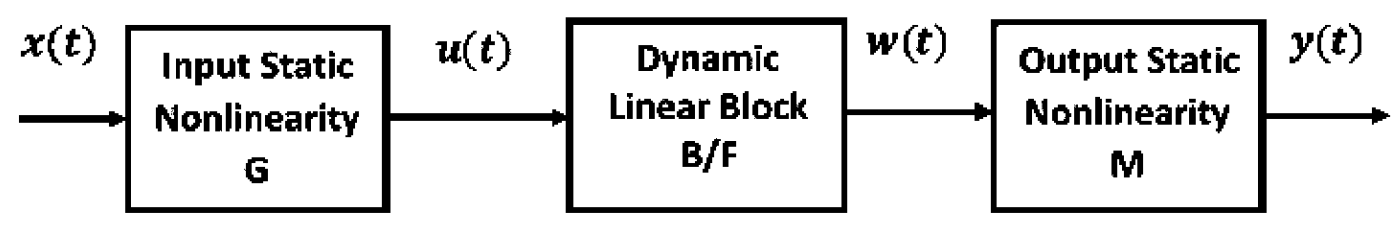

Figure 3.12: The schematic block-diagram of the nonlinear Hammerstein-Wiener estimation model

The static nonlinearity of the first block is characterized by: 


$$
\begin{aligned}
u(t) & =G(x(t)) \\
& =a_{1} x(t)+a_{2} x^{2}(t)+a_{3} x^{3}(t)+. .+a_{n} x^{n}(t)
\end{aligned}
$$

The output of the second block is given by:

$$
w(t)=\frac{B\left(z^{-1}\right)}{F\left(z^{-1}\right)} u(t)
$$

where

$$
\begin{aligned}
& B\left(z^{-1}\right)=b_{1} z^{-1}+b_{2} z^{-2}+b_{3} z^{-3}+. .+b_{n} z^{-n} \\
& F\left(z^{-1}\right)=1+f_{1} z^{-1}+f_{2} z^{-2}+f_{3} z^{-3}+. .+f_{n} z^{-n}
\end{aligned}
$$

For the third nonlinear block,

$$
\begin{aligned}
y(t) & =M(w(t)) \\
& =a_{1} w(t)+a_{2} w^{2}(t)+a_{3} w^{3}(t)+. .+a_{n} x^{n}(t)
\end{aligned}
$$

In Equation 2, $G$ is a nonlinear function that transforms the input data $x(t)$ into $u(t)$. For the dynamic linear block, $W$ in Equation 3 represents a linear transfer function. $B\left(z^{-1}\right)$ and $F\left(z^{-1}\right)$ are the numerator and the denominator of the transfer function (similar to the polynomials). Finally, $M$ in Equation 6 represents a nonlinear function which maps the linear output of the second block to the model output $y(t)$. Three networks of Hammerstein-Wiener models with different segment values were selected in this work using MATLAB system identification toolbox. A piecewise-linear function with several segments and one-dimensional polynomial of third-order were used to represent both input and output of the nonlinearity. These networks were able to 
model the sensor more dynamically with smooth curves and very short simulation time. For the linear part, a linear transfer function that has four zeros and five poles was chosen. This was found to be sufficient to characterize the linear behavior of the sensor. In the $\mathrm{x}$ direction, a Hammerstein-Wiener model with one input and one output

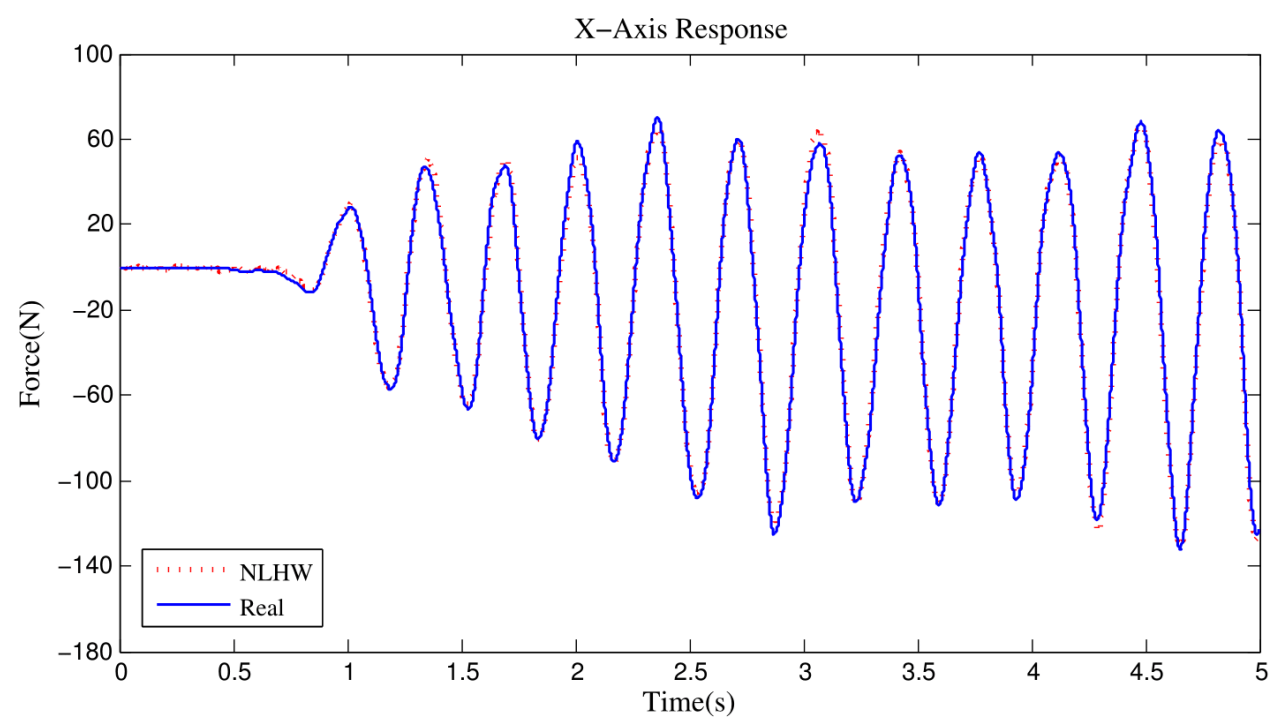

Figure 3.13: A comparison between Hammerstein-Wiener model of the $\mathrm{x}$-axis and the real data. The fitting value was improved by $10 \%$ compared to the previous linear models.

was utilized. The input nonlinearity estimator consisted of a piecewise-linear function of ten segments with unspecified break-points. In the middle block, a linear transfer function with the orders of $n b=4$ (number of zeros), $n f=5$ (number of poles) and $n k=0$ (the input delay) was applied. For the output nonlinearity estimator, a one dimensional third-order polynomial was selected. The model gave the best curve fit with fitting value of $94.12 \%$ and with an RMSE of $3.05 \mathrm{~N}$. The errors were reduced by 42.01\% compared to the best linear estimation models. Figure 3.13 shows the results of the model in comparison to the ATI 6-axis F/T sensor.

For the y-axis, the input and output of the nonlinear blocks were composed of piecewise-linear functions with ten and six segments respectively. A linear transfer 
function with the orders of $n b=4, n f=5$ and $n k=0$ was used for the linear part of the model. These parameters were found to be effective in minimizing the errors. The results showed a fitting value of $92.25 \%$. The root mean square error was minimized from $5.75 \mathrm{~N}$ to $3.72 \mathrm{~N}$. Figure 3.14 illustrates the dynamic response of the y-axis.

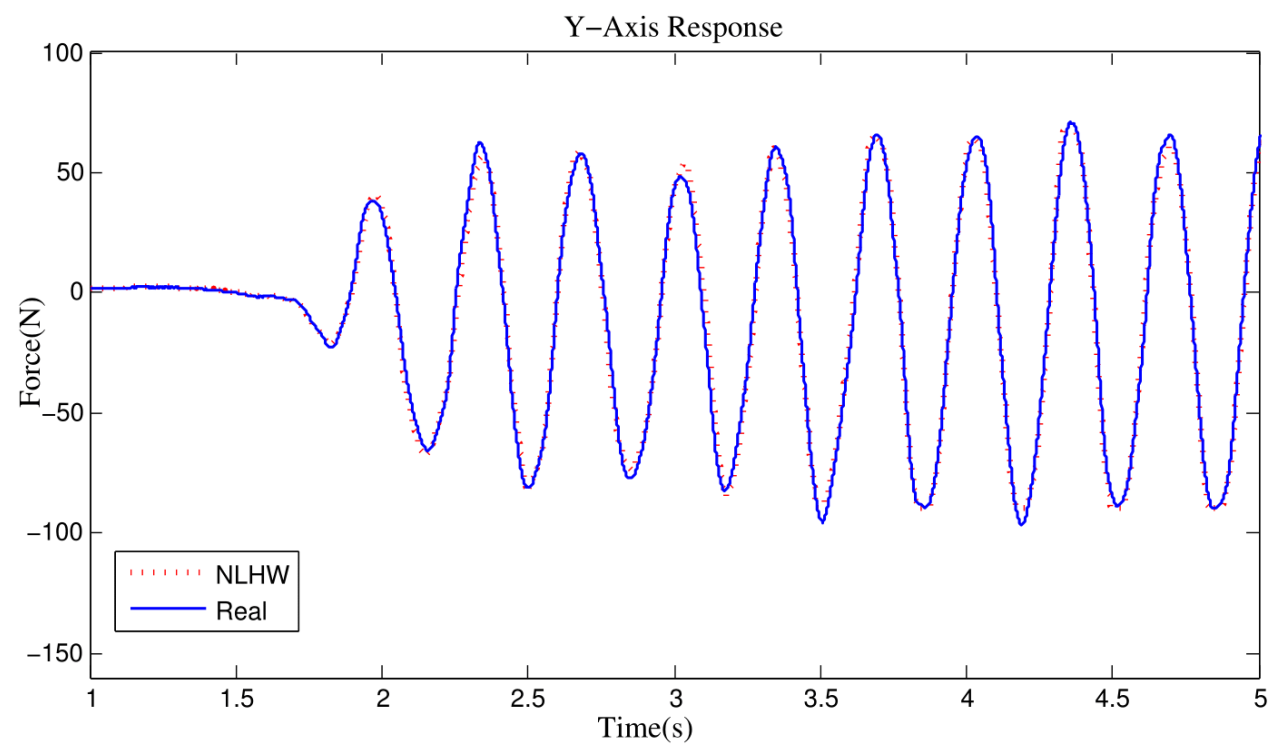

Figure 3.14: The optimized results of the y-axis using Hammerstein-Wiener model compared to the real data.

In the z-axis, the NLHW included a piecewise-linear function for the input nonlinearity and a linear transfer function with the orders of $n b=5, n f=6$ and $n k=0$. The output nonlinearity consisted of a piecewise-linear function with six segments. The results were relatively more accurate than the $y$-axis with a fitting value of $93.20 \%$. The RMSE was reduced from $23.90 \mathrm{~N}$ to $14.78 \mathrm{~N}$ and the maximum error was decreased from $10.14 \%$ to $7.78 \%$. The dynamic response of the sensor along the z-axis is depicted in Figure 3.15.

The experimental results of the compliant 3-axis force sensor are summarized in table II. It includes a comparison between the real data of the ATI 6-Axis F/T sensor and the measured data with respect to: the type of estimation model, best fitting 
value, RMSE and the percentage of the RMSE. The results of the z-axis showed the highest level of accuracy with a small percentage of RMS error (1.92\%) compared to those of the $\mathrm{x}(2.30 \%)$ and the $\mathrm{y}$-axis $(4.15 \%)$. This essentially stems from the linear relationship achieved during the static loading test, validating the nonlinearity cancellation phenomenon described previously. In the $\mathrm{x}$ and $\mathrm{y}$ axes, the lack of the linear correlation led to less accurate results.

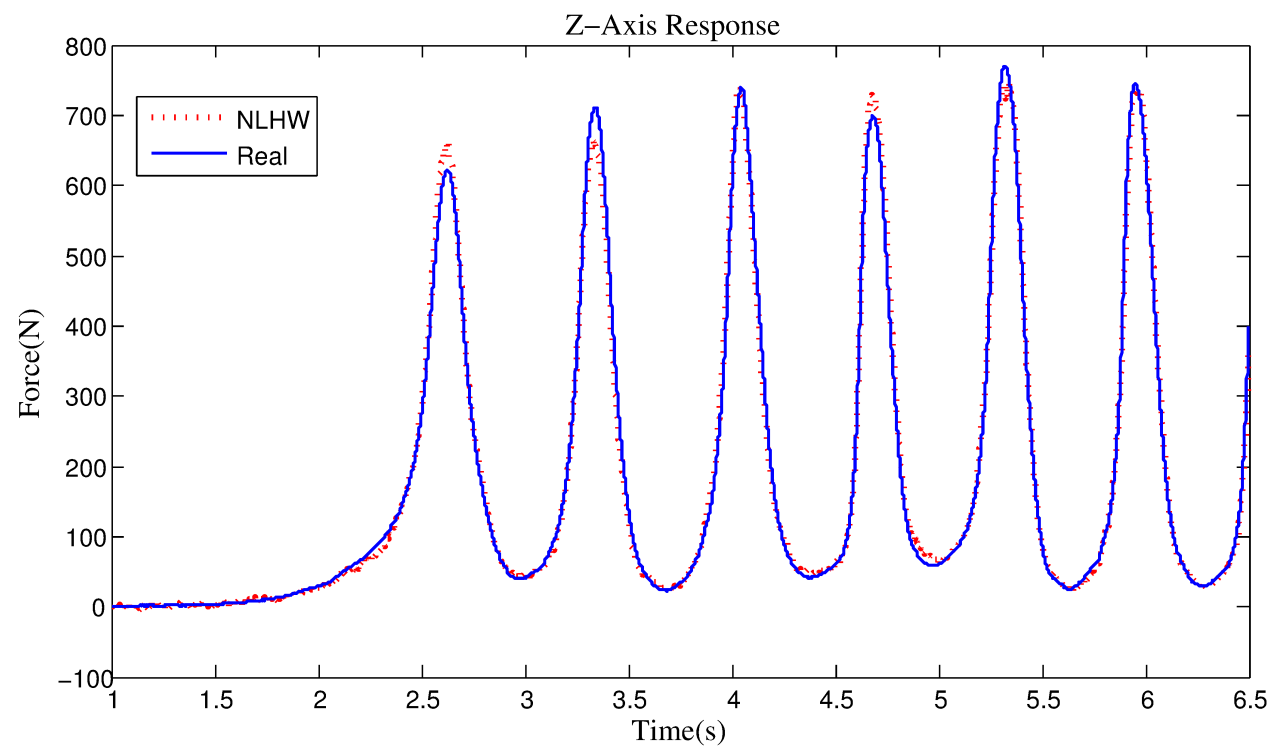

Figure 3.15: A comparison between the Hammerstein-Wiener model used to model the $\mathrm{z}$-axis and the real data of the ATI 6-axis F/T sensor

The comparison also incorporates the performance of the quadratic models that were achieved form the static test data in Figure $3.7(\mathrm{~d})$. In this case, the models were used to characterize the dynamic test data along each axis. However, the RMS errors were relativity high at $51.77 \mathrm{~N}(6.72 \%)$ in the z-axis, $13.06 \mathrm{~N}(9.84 \%)$ and $15.18 \mathrm{~N}(16.93 \%)$ in the $\mathrm{x}$ - and $\mathrm{y}$-axis respectively compare to the dynamic estimation models. This is mainly because of the dynamic behaviours caused by the mass and viscoelastic properties of the flexible material used; therefore, it was required for this 
Table 3.2: The experimental results of the proposed 3-axis fiber-optic force sensor as a comparison to the real data of the ATI 6-axis $\mathrm{F} / \mathrm{T}$ sensor.

\begin{tabular}{|c|c|c|c|}
\hline \multicolumn{5}{|c|}{ X-Axis } \\
\hline Model Type & Fitting Value & RMSE & RMSE $\%$ \\
\hline Hammerstien-Wiener & $94.12 \%$ & $3.05 \mathrm{~N}$ & $2.30 \%$ \\
\hline Transfer Function & $87.40 \%$ & $6.54 \mathrm{~N}$ & $4.93 \%$ \\
\hline Auto-Regressive & $85.88 \%$ & $7.32 \mathrm{~N}$ & $5.46 \%$ \\
\hline Output-Error & $84.04 \%$ & $8.28 \mathrm{~N}$ & $6.25 \%$ \\
\hline Quadratic/Static & $\mathrm{R}^{2=} 0.94$ & $13.06 \mathrm{~N}$ & $9.84 \%$ \\
\hline \multicolumn{5}{|c|}{$\mathrm{Y}-\mathrm{Axis}$} \\
\hline Hammerstein-Wiener & $92.25 \%$ & $3.72 \mathrm{~N}$ & $4.15 \%$ \\
\hline Transfer Function & $89.19 \%$ & $5.21 \mathrm{~N}$ & $5.81 \%$ \\
\hline Auto-Regressive & $87.01 \%$ & $6.24 \mathrm{~N}$ & $6.94 \%$ \\
\hline Output-Error & $86.86 \%$ & $6.32 \mathrm{~N}$ & $7.08 \%$ \\
\hline Quadratic/Static & $\mathrm{R}^{2}=0.86$ & $15.18 \mathrm{~N}$ & $16.93 \%$ \\
\hline \multicolumn{5}{|c|}{$\mathrm{Z} \%$-Axis } \\
\hline Hammerstein-Wiener & $93.20 \%$ & $14.78 \mathrm{~N}$ & $1.92 \%$ \\
\hline Transfer Function & $91.72 \%$ & $20.27 \mathrm{~N}$ & $2.63 \%$ \\
\hline Output-Error & $89.01 \%$ & $23.91 \mathrm{~N}$ & $3.10 \%$ \\
\hline Auto-Regressive & $88.97 \%$ & $23.98 \mathrm{~N}$ & $3.11 \%$ \\
\hline Quadratic/Static & $\mathrm{R}^{2}=0.95$ & $51.77 \mathrm{~N}$ & $6.72 \%$ \\
\hline
\end{tabular}

class of sensors to compensate for nonlinear behaviours natural to large strains and deflections.

\subsection{Conclusion}

In this chapter, a high-accuracy, low-cost, compliant 3-axis fiber-optic force sensor based on light intensity modulation was designed, manufactured and calibrated 
successfully. The sensor was capable of measuring the magnitude and the direction of the applied normal and shear forces simultaneously with a potential to adjust the sensing range. Due to its flexibility and wide force measurement range, the sensor can be used in different biomedical applications such as measuring the total ground reaction force, collision detection and applications where compliance of the sensing element is needed and rigidity is not suitable, such as human-robot interaction. Both static and dynamic tests were performed to study the sensor's characteristic response in different loading conditions.

In the dynamic tests, linear and nonlinear system identification models have been applied and both showed satisfactory results. Since the sensor exhibited nonlinearity which is mostly attributed to the elastic material behavior, the Hammerstein-Wiener estimation model was necessary to characterize both the linearity and nonlinearity of the sensor at the same time. The model gave the best results with fitting values of: $F x=94.12 \%, F y=92.25 \%$ and $F z=93.20 \%$ compared to the linear estimation models such as the fifth-order transfer function that gave lower accurate results: $F x=$ $87.40 \%, F y=89.19 \%$ and $F z=91.72 \%$ of fitting values. The sensor was able to address the undesired cross-talk effects that current available sensors suffer from. The maximum cross-talk errors were ranging from $0.37 \%$ to $4.01 \%$, making it feasible to simultaneously measure the force along the three axes without the need to use cross-talk compensation. 


\title{
Chapter 4
}

\section{A 6-DoF, Wearable, Compliant Shoe Sensor for Total Ground Reaction}

\section{Measurements}

\begin{abstract}
In this chapter, we present a compliant six-axis sensor for total ground reaction measurement, the calibration methodology, and preliminary data. The sensor is intended for gait analysis and is designed to have minimal effect on natural gait. A triaxial optical force sensor is combined with an array of pressure sensing films to form a wearable compliant six-axis force/moment sensor. Two sensor units were developed for the toe and heel and used in two types of experiments: stepping on the sensors that are mounted on the ground, and attaching the sensors under the shoe while walking. The data from the sensors are compared with measurements obtained from a standard
\end{abstract}


force plate. The deflections induced by the sensor compliance exhibit a slight nonlinear force-deflection relation. Regardless of the nonlinear effects, the sensor is accurately calibrated with a linear least squares method. To see how well these nonlinearities could be calibrated for, a nonlinear calibration with a neural network was used. For the sensors attached to the floor, a linear calibration achieved an RMSE of $4.49 \%$ while the neural network achieves $2.68 \%$. For the wearable sensor, the linear calibration RMSE was $9.39 \%$ and the neural network RMSE was $5.21 \%$. When the orientation data of the sensors (measured by a motion capture system) was added as an input to the neural network calibration, the RMSE error was reduced to $3.25 \%$.

\subsection{Introduction}

Falls are a significant issue for the elderly. About one in three people over the age of 65 will experience a fall every year, with the rate increasing with age, and quarter of those who fall have a serious injury $[165,166]$. In 2012, the United States had a direct medical cost of $\$ 616.5$ million for fatal and $\$ 30.3$ billion for non-fatal fall injuries [167]. Impaired balance and mobility have been shown to be risk factors for falls [168] showing the importance of monitoring and improving balance and mobility for fall prevention.

Gait analysis is a method to help better understand falls and help in the development of a personalized assistive fall prevention devices. Performing gait analysis requires a posture sensing system and a measurement of the complete ground reaction forces and moments; these sensors should not disturb natural gait. A desired gait measurement system must be able to be used inside and outside a laboratory setting for use in a fall detection and prevention system. In this chapter, we develop a six-axis, wearable shoe sensor to be used to measure forces and moments in gait. The sensor 
is lightweight, compliant, inexpensive, and easily modifiable to minimize its effect on gait.

Force plates in laboratories are the traditional method for gait analysis and capable of accurately measuring the ground reaction forces and moments, but are restricted to laboratory use. A laboratory typically has one or two force plates fixed to the floor for a maximum of one or two recorded steps per trial. The foot must be placed fully within the bounds of the force plate. Force plate targeting to consciously attempt to step within the force plate may have an affect on some joint kinematics [169] and may disturb gait. Force plates are unable to provide the forces and moments acting on each foot while both feet are on the same force plate. These limitations indicate the need for an ambulatory, wearable sensor.

In terms of ambulatory gait measurement systems, pressure insoles such as the F-Scan are commonly used in various biomechanical studies to track centre of pressure, gait events, and to assess walking [170-172]. An insole containing only eight pressure sensors was able to measure walking speed, manner of walking, and gait rhythm [173]. Pressure insoles are known to suffer from error under certain conditions. The pedar-X was found to have creep behaviour in which pressure reading increase several hours of measurement. The insole also changes sensitivity depending on contact area and temperature [174]. New high accuracy plantar pressure measurment technologies have been developed that use fibre Bragg grating and 3D printing [175]. These pressure measurement systems resolve the limitations of force plates but cannot measure the full ground reaction force, only the vertical force. They also do not directly measure the true ground reaction force but the pressure inside the shoe, which is affected by shoe tightness, and other factors. 
Regression, linear, and nonlinear mapping techniques have been developed to estimate shear forces from pressure sensor matrix $[176,177]$ with only moderate success in estimating the complete ground reaction forces. For example, both [176] and [177] had a significantly lower accuracy in the medial-lateral shear force direction. A poor correlation value for predicting the medial-lateral direction forces from pressure insole data using regression techniques was also cited in [176]. The pressure matrix has no independent measurement of the shear force, making estimates a difficult process.

An instrumented shoe was developed that mounts two ATI Mini45 load cells to the shoe [178]. This sensor was able to accurately measure the forces and moments under the foot in a wearable fashion; however, the ATI sensors were rigid, which may distract from natural gait and cause discomfort and may have weight concerns. Attaching heavy weights to the foot distracts from natural gait and may cause fatigue for the wearer. The cost of the 6-axis load cells is too high to be viable for a personalized fall decection and prevention device, restricting such a configuration for laboratory use. Alternative multiaxis wearable ground reaction force sensors have been developed in reasearch but currently are large and suffer from crosstalk error [179].

Wearable sensors for gait have been proposed to remove the restrictions of a force plate [180-182], but are limited to only measuring three forces (one vertical and two shear). These sensors, however, may have sensitivity to moments that cannot be eliminated without additional measurements. The moments that occur in gait could affect the force measurements from the sensor. The lack of a moment measurement also means these sensors cannot measure the centre of pressure. It is desirable to have a sensor that can both measure ground reaction forces and moments, as centre of pressure is a common measure used in balance [183, 184]. In [185], three small triaxial force sensors are mounted on a board to make a small wearable force plate, two of 
which are used to instrument the foot. The moment acting on the torque sensors affect the force measurments on the plate, increasing measurement error.

In [180], we developed a compliant fibre optic based sensor for triaxial force measurement. We propose a modified system that transforms the sensor into an enhanced wearable six-axis sensor system that is both lightweight and compliant. It combines two different shoe sensor technologies: the triaxial force sensor and the pressure sensor matrix. The new sensor uses an intensity-based fibre optic sensor as the triaxial force sensor and FlexiForce A301 force sensitive resistors to capture full force and moment profile of gait with minimal gait disturbance. The original sensor in [180] is affected by nonlinearities; the addition of the FlexiForce sensors helps to improve the linearity of the new sensor package.

The compliance of the sensor [180] due to the blocks of rubber integrated in the sensor structure is very desirable for gait measurement, but large deflections also lead to nonlinear behaviours requiring special calibrations such as the one in [186]. The sensor detects force based off of deflection from the rubber, which is a nonlinear relationship. The force sensor thus is affected by nonlinearities. In this chapter we examine the effect of the nonlinearities by using both a linear calibration and a nonlinear calibration.

\subsection{Sensor Design}

The sensor has two modules: module (1) is a fibre optic, compliant, triaxial force sensor; module (2) is a thin plate equipped with four round pressure sensing films (part (9) in Figure 4.1) to generate outputs that are sensitive to moments. The sensor system expands on the fibre optic force sensor in [180] by adding pressure sensors to 
improve its moment sensitivity. Two of these modules are proposed to be mounted on each shoe as an outsole.

\subsection{Fiber-Optic Shear Force Sensor}

The sensor system uses a previously developed triaxial fibre optic force sensor [180]. This sensor detects force using light intensity modulation by reflectance. The sensor consists of two rigid layers of 3D printed PLA connected by a compliant material, Mold Max 60 rubber. One layer has reflective surfaces and the other layer houses the LEDs, TSL250R light-to-voltages (LTVs), and fibre optics. When a force is applied to the sensor, the rubber deforms and the top layer moves with respect to the bottom layer. This increases or decreases the intensity of the light detected by the LTVs. The sensor seen in Figure 4.2 is lightweight, low-cost, and compliant. It has a sensing range applicable to the magnitude of forces in human gait. Its flexibility allows for more natural gait than would be possible with a rigid sensor.

The triaxial force sensor design was modified to accommodate the FlexiForce sensors. Force sensitive resistors were selected for their size and compatibility with the triaxial sensor. The force sensitive resistors are able to detect moment when arranged in an array and are unable to sense shear. When a moment is applied to the sensor, the force distribution across the FlexiForce sensors changes, which allows for the measurement of moment. The triaxial sensor has no moment sensing ability but is able to sense normal and shear forces. The top layer of the triaxial sensor was modified to include four pucks of nine millimetre diameter as attachment points for the FlexiForce sensors. These pucks ensure that the only load path through the sensor is through the FlexiForce sensors. The modified sensor can be seen in Figure 4.1. To 


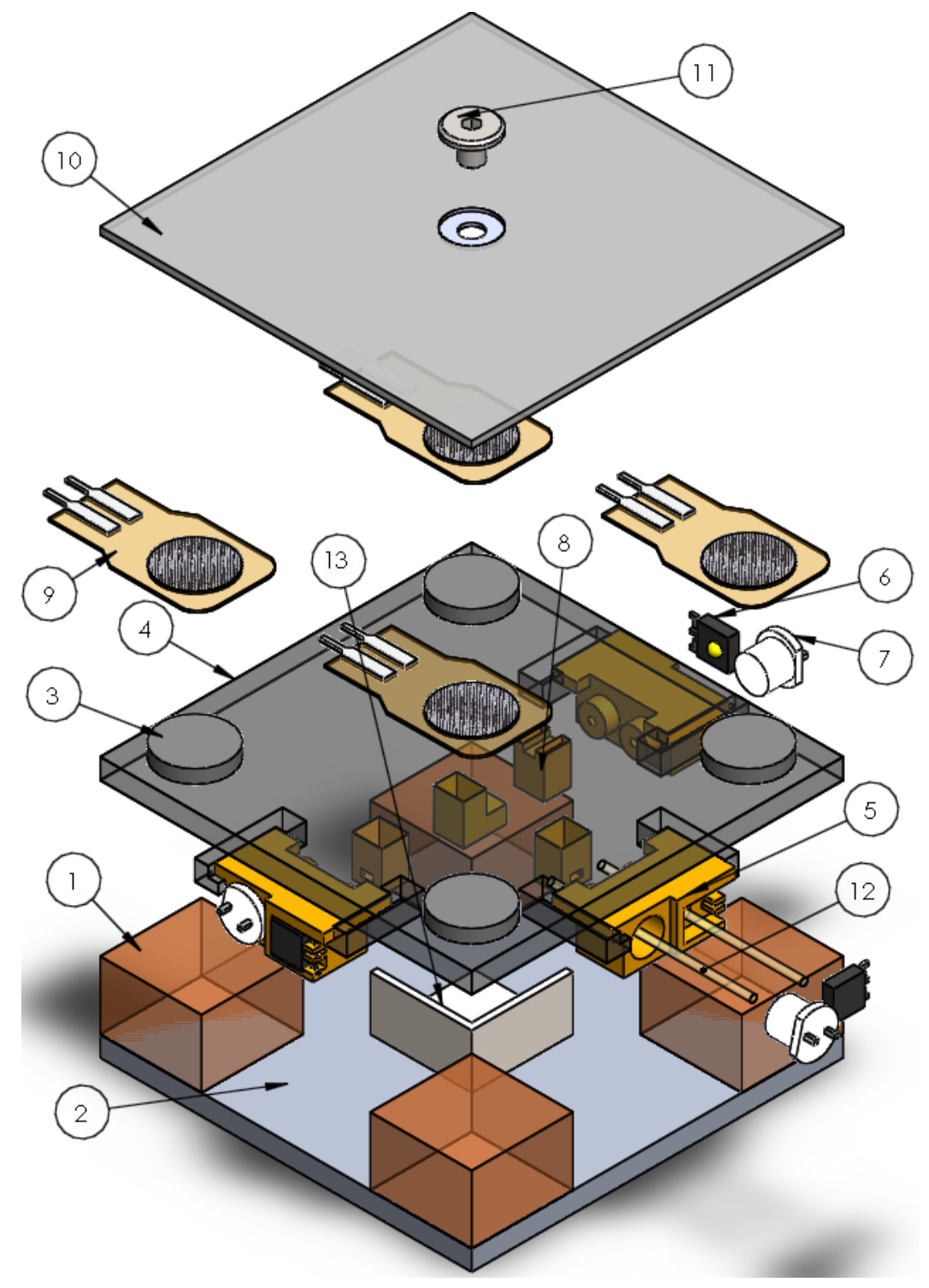

Figure 4.1: Structure of the proposed sensor. (1) Silicon rubber block. (2) Bottom plate (PLA). (3) Higher section to mount FlexiForce sensors. (4) Top plate. (5) LED and LTV holder. (6) TSL250R (LTV ). (7) OVLEW1CB9 (LED). (8) Fibre guider. (9) FlexiForce sensors (A301). (10) Top layer (11) Screw to precompression of the FlexiForce. (12) Optical fibres. (13). Light reflector. 
improve portability, the fiber optic sensor was reconfigured to use a nine volt battery as a power source.

FlexiForce A301 and the TSLR250 have a $0.36 \% /{ }^{\circ} \mathrm{C}$ and a $0.08 \% /{ }^{\circ} \mathrm{C}$ sensitivity to temperature change respectively, making the proposed sensor slightly affected by temperature. Both the FlexiForce sensors and the triaxial fiber optic sensor are capable of measuring the force in the Z-direction. This provides redundancy in measurements. The Z-axis reflective surface along with the corresponding LTV and LED were removed from the fiber optic sensor to save battery life, minimize output channels, and to create potential reducing the sensor thickness by avoiding the need to bend fibers.

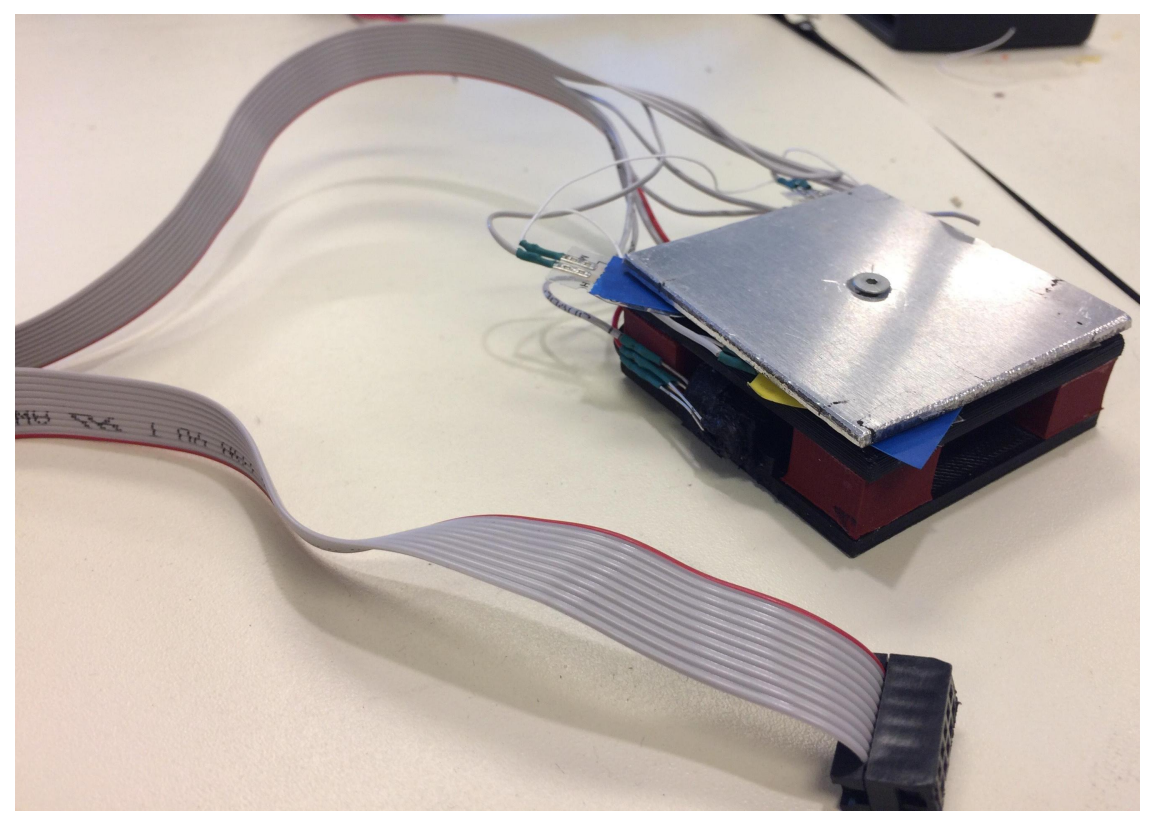

Figure 4.2: The prposed sensor unit separated from the other related electronics. The LED and LTV are shown on the sensor side. 


\subsection{FlexiForce Sensors}

The fiber optic sensor is able to accurately determine forces in the vertical and two shear directions [180], but has sensitivity to moments. It is impossible to determine the full force and moment profile with only the three outputs provided by the sensor. Additional sensing elements, four FlexiForce A301, were added to create new outputs sensitive to moment and to help determine the Z-direction force and the moments. The FlexiForce sensors were placed in each of the corners on top of the raised sections (part (3) in Figure 4.1) on the modified top layer of the fiber optic sensor. The FlexiForce sensors were taped down to the raised sections on one side and covered with a protective layer of tape on the other, seen in Figure 4.3. An additional aluminum plate was added to the top of the sensor to create the load bearing surface. This brought the final height of the system to be $2.1 \mathrm{~cm}$, with a $5.5 \mathrm{~cm}$ length and width.

The aluminum plate is screwed into the top layer of the fiber optic sensor with a countersunk screw that sits below the surface of the plate. The hole in the plate is large enough to freely move along the screw, thus not including the screw as a load path unless the sensor is in tension. This screw provides some precompression on the FlexiForce sensors. The FlexiForce sensors are incapable of sensing tension, but certain applications of moments on the sensor can cause tension scenarios for individual FlexiForce sensors. Therefore, the system allows for the application of precompression to detect tension scenarios at the cost of having less compression force range. The screw connects the top aluminum plate and the top of the fiber optic sensor parts ((10) and (4) in Figure 4.1), taking all shear load from the FlexiForce layer.

A voltage is obtained from the FlexiForce resistors via Tekscan's recommended 


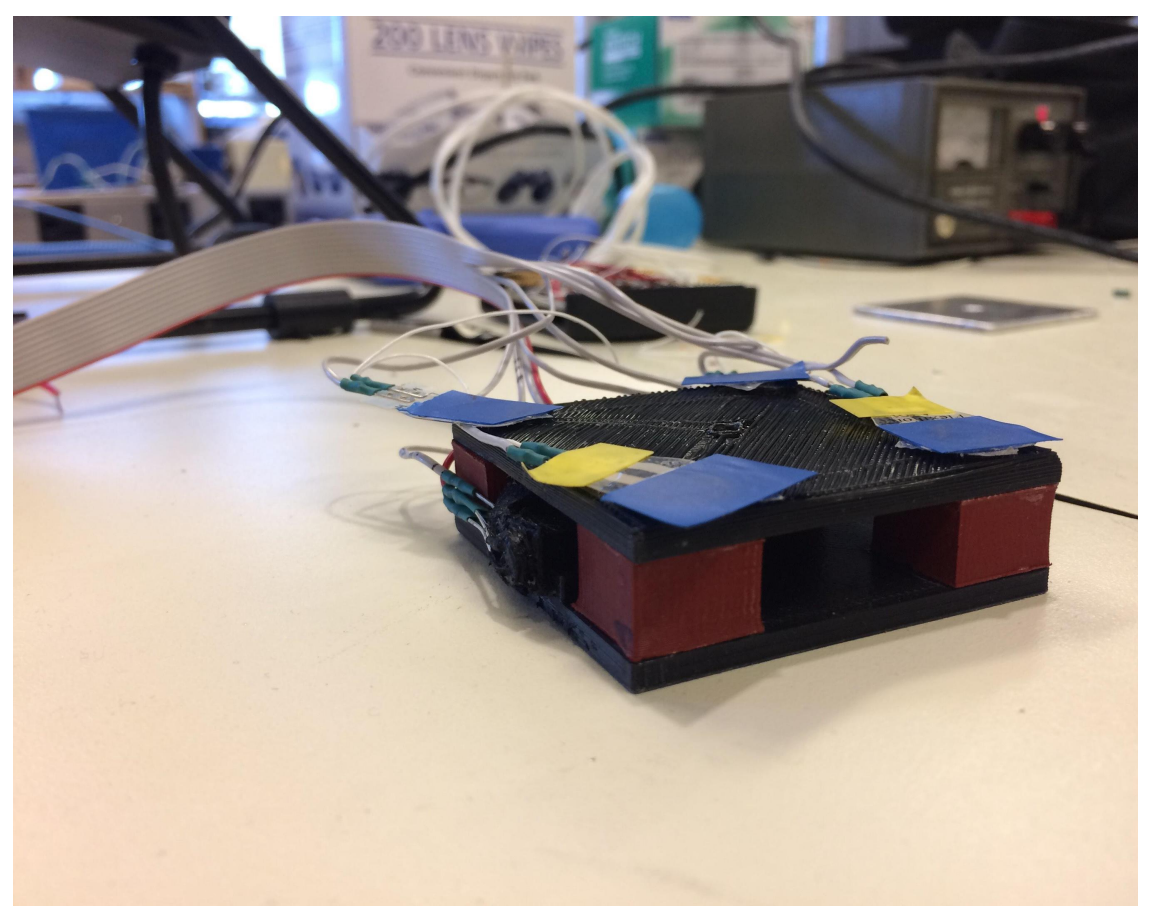

Figure 4.3: Sensors unit with top aluminum plate removed, exposing the FlexiForce sensors (covered with blue electrical tape for protection)

circuit using a $10 \mathrm{k} \Omega$ resistor in place of the potentiometer. This configuration makes the output voltage linear with respect to force.

This circuit is located in a BusBoard 1593K Box mounted on the ankle, seen in Figure 4.4. The sensors are powered by a nine volt battery, also seen on the box. This system can be used for ambulatory gait analysis if used with a mobile DAQ solution. Data is filtered through a $72 \mathrm{~Hz}$ 1st order Butterworth hardware anti-aliasing filter and to a NI PCI-6229 DAQ..

\subsection{Experimental Results}

Two main sets of experiments were arranged to evaluate the proposed sensing technology. In the first series, two sensors were mounted on a Bertec force plate and 
were walked across. In the second set, two sensors were mounted to the bottom of a shoe, and a step was taken across the force plate. Optotrak markers were used to determine the position and orientation of the sensors relative to the ground. For this wearable enhanced prototype, only gait data is used since the intended application is for gait analysis and total ground reaction force measurements

\subsubsection{Floor-mounted experiments}

To verify the new sensor's ability to measure six-axis forces and moments in gait, the two sensor systems were tested while attached to the ground. The sensors were mounted with tape to a Bertec (Ohio, United States) FP-4060-07-1000 force plate, a six-axis force and moment transducer with $0.5 \mathrm{~N}$ accuracy for Z-direction force. The sensors were $10.5 \mathrm{~cm}$ apart in the Y-direction of the plate and aligned in the Z-direction. Gait data was recorded as a person walked across the force plate, stepping on the sensors. Data was recorded with Bertec AM6800 Amplifier digital output with Bertec Digital Acquire and an NI PCI 6229 DAQ at $1 \mathrm{kHz}$. The six-axis data from the force plate was used as a reference to calibrate the sensor. Two methods were used to process the data, linear and nonlinear.

\subsubsection{Least squares regression}

Linear regression was used as a first approach to calibrate the sensor [187, 188] as preliminary tests indicated it had a highly linear response. A total of ten steps around 40,000 data points were collected. As the user only plays the role of applying force and does not affect the physics of the sensor, a small sample of steps were used. The sensor behaviour was found to be independent of the user and user's gait speed. Gait data 


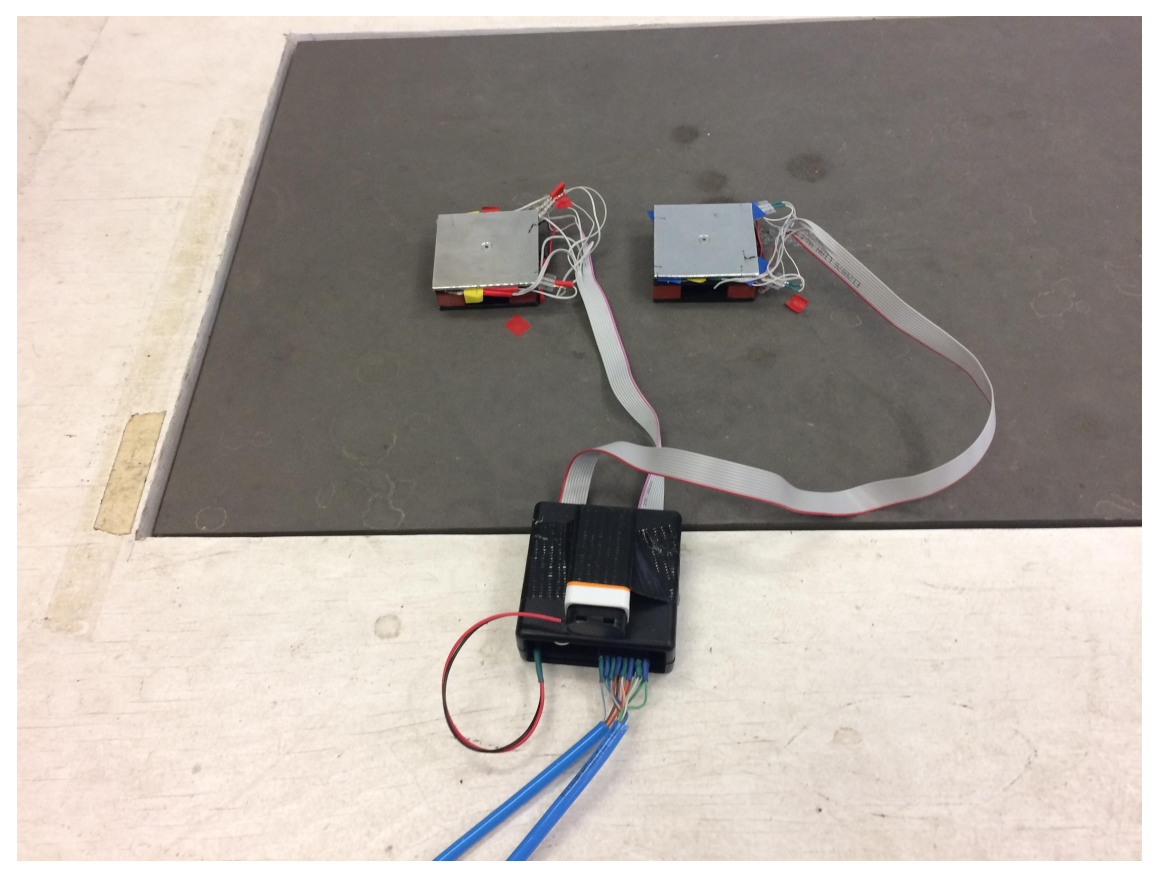

Figure 4.4: Two Sensor units are affixed on the force plate

is shown instead of a machine-applied load in order to show this sensor's ability in its intended application. The calibration results can be seen in Figure 4.5 and Figure 4.6. High accuracy for each axis was achieved, with a RMS error percent of $4.49 \%$. The RMS error, RMS percent, and a correlation coefficient were calculated. The error is shown in Figure 4.8. Detailed results for each axis can be found in Table 4.1. The relatively low RMS error of the least squares regression implies that the sensor has highly linear characteristics. The FlexiForce sensors were set up to provide a linear voltage with force with a hysteresis of less than $4.5 \%$. The fiber optic sensor was noted to have nonlinearities due to the flexible material but still has mostly linear behaviour seen in Figure 4.7 where hysteresis effects are visible but not major. Addition of the FlexiForce sensors helps to remove the effects of moment on the fiber optic sensor's shear force measurement.

The least squares regression was able to get a highly accurate estimate of the 
forces and moment in gait, especially with respect to the Z-direction force and $\mathrm{X}$ and Y-direction moments. These forces and moments rely mostly on the FlexiForce measurements, which have a linear response and thus have high accuracy when processed with a linear calibration. A less accurate result is seen in the $\mathrm{X}$ - and $\mathrm{Y}$ forces and the Z-moment direction. These errors can be attributed to a low magnitude of forces and moments, resulting in a poor signal to noise ratio. Changing the gain of these axes to accommodate for the lower force range would result in a more accurate measurement. The $\mathrm{X}$ - and $\mathrm{Y}$ - forces are mainly determined from the fiber optic sensor, and are expected to suffer more from nonlinearities than the FlexiForce based moments. These measurements were improved with a nonlinear calibration. The array of FlexiForce sensors lie in a plane, and thus have no sensitivity to the Z-moment. The Z-moment information comes from the shear sensor's sensitivity to moment. The Z-moment measurement thus is less linear than other moments and the measurement was improved by applying a nonlinear calibration.

\subsubsection{Neural network regression}

Despite the linear results, the shear sensor is affected by a nonlinear force-displacement relationship for the rubber, a nonlinear light intensity-displacement relationship, and rotation of the reflective surfaces under moment load. A nonlinear approach, a neural network, was used for sensor calibration in attempt to achieve a higher accuracy. A neural network is an architecture that uses optimized interconnected neurons to predict an output. In this case, the network acts as a nonlinear regression technique. The neural network is not used as a complete optimized nonlinear solution but as proof that the results can be improved with a nonlinear fit. The results can be found in Figure 4.5, Figure 4.6 and Figure 4.9. 
A neural network with one hidden layer with ten neurons was trained using the same data set with a $70 \%$ training, $15 \%$ validation, and $15 \%$ testing set split. The overall network consisted of an input layer of twelve neurons (eight FlexiForce, four LTVs), a hidden layer of ten neurons with a tansig activation function, and an output layer of six neurons with a linear activation function. Levenberg-Marqardt backpropagation was used to train the network. with an initaial my of 0.001 and a minimum gradient of $10^{-7}$. Detailed results quantifying the fit of each axis can be found in Table 4.1.

The neural network was able to achieve a higher prediction accuracy than the least squares method, with an average RMS error percent of $2.68 \%$. This is expected as the neural network is able to fit the nonlinear characteristics of the sensor. The neural network improves the correlation coefficient for the shear forces and Z-moment, which are measurements that would be affected by the nonlinearity of the rubber. Thus, the neural network is able to calibrate for nonlinear measurements. Both nonlinear and linear techniques were used to calibrate the sensor with high accuracy in this configuration. A comparison of the force accuracy between the six-axis sensor and the triaxial sensor in [180] is shown in Table 4.2.

\subsubsection{Shoe-mounted experiments}

The sensor provided very good results for the floor mounted configuration. However, as a floor mounted sensor, it suffers from the same issues as a force plate. The main benefit of this sensor is that it is wearable and usable outside of a laboratory setting. The next experiment, seen in Figure 4.10 on page 90, tested the sensor's performance in its intended wearable configuration.

The sensor was attached to the shoe for this test. Two sensor units were used 
Table 4.1: Experimental results of 6-axis sensor mounted on floor during gait

\begin{tabular}{|c|c|c|c|}
\hline \multicolumn{4}{|c|}{$\mathrm{X}$-Axis Force } \\
\hline Model Type & R-squared $\%$ & RMSE & RMSE \% \\
\hline Linear & $96 \%$ & $2.87 \mathrm{~N}$ & $6.80 \%$ \\
\hline Neural Network & 0.99 & $1.20 \mathrm{~N}$ & $2.86 \%$ \\
\hline \multicolumn{4}{|c|}{ Y-Axis Force } \\
\hline Model Type & R-squared \% & RMSE & RMSE \% \\
\hline Linear & $94 \%$ & $3.62 \mathrm{~N}$ & $5.41 \%$ \\
\hline Neural Network & $98 \%$ & $1.685 \mathrm{~N}$ & $2.50 \%$ \\
\hline \multicolumn{4}{|c|}{ Z-Axis Force } \\
\hline Model Type & R-squared \% & RMSE & RMSE \% \\
\hline Linear & $99 \%$ & $15.04 \mathrm{~N}$ & $2.77 \%$ \\
\hline Neural Network & 0.99 & $4.60 \mathrm{~N}$ & $0.84 \%$ \\
\hline \multicolumn{4}{|c|}{$\mathrm{X}$-Axis Moment } \\
\hline Model Type & R-squared \% & RMSE & RMSE \% \\
\hline Linear & $99 \%$ & $1.11 \mathrm{~N} \cdot \mathrm{m}$ & $1.96 \%$ \\
\hline Neural Network & $99 \%$ & $1.02 \mathrm{~N} \cdot \mathrm{m}$ & $1.95 \%$ \\
\hline \multicolumn{4}{|c|}{ Y-Axis Moment } \\
\hline Model Type & R-squared $\%$ & RMSE & RMSE \% \\
\hline Linear & $99 \%$ & $0.56 \mathrm{~N} \cdot \mathrm{m}$ & $2.75 \%$ \\
\hline Neural Network & $99 \%$ & $0.42 \mathrm{~N} \cdot \mathrm{m}$ & $2.08 \%$ \\
\hline \multicolumn{4}{|c|}{ Z-Axis Moment } \\
\hline Model Type & R-squared\% & RMSE & RMSE \% \\
\hline Linear & $99 \%$ & $0.29 \mathrm{~N} \cdot \mathrm{m}$ & $7.23 \%$ \\
\hline Neural Network & $94 \%$ & $0.24 \mathrm{~N} \cdot \mathrm{m}$ & $5.85 \%$ \\
\hline
\end{tabular}




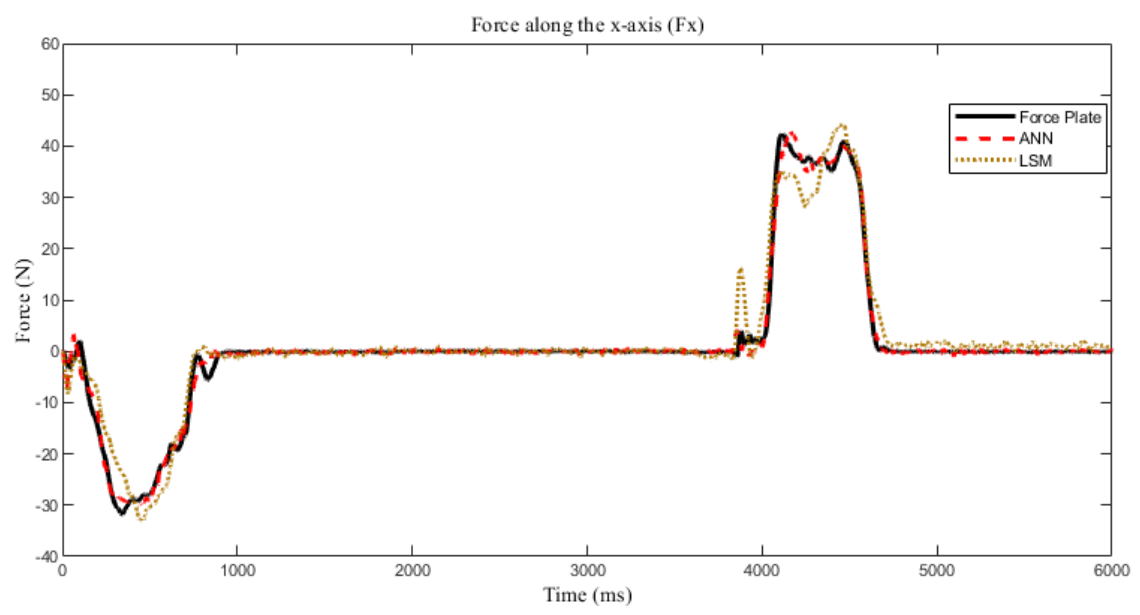

(a)

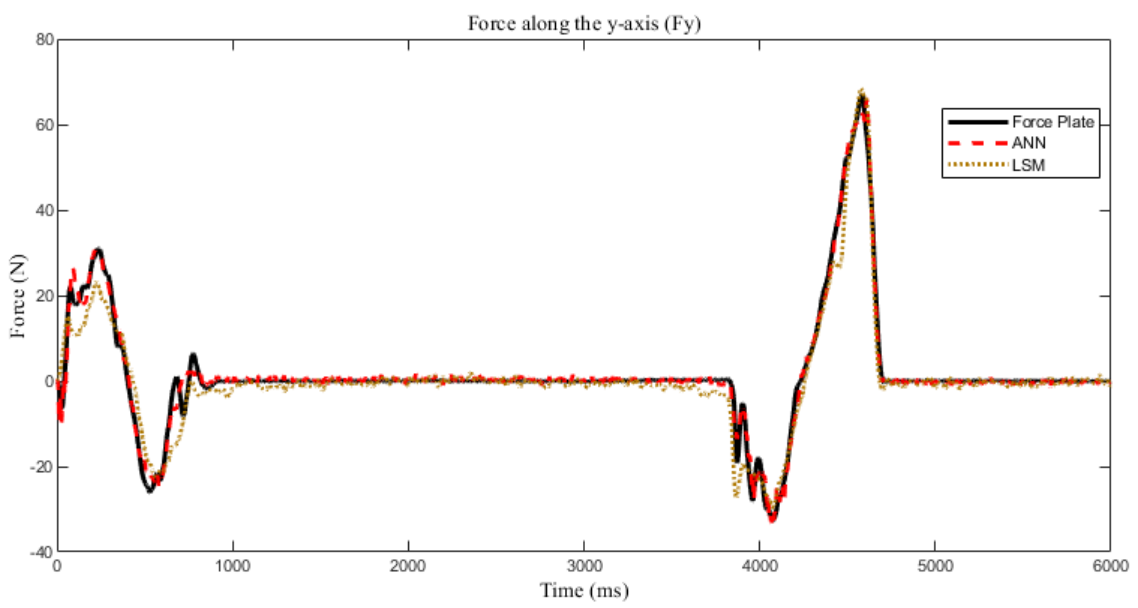

(b)

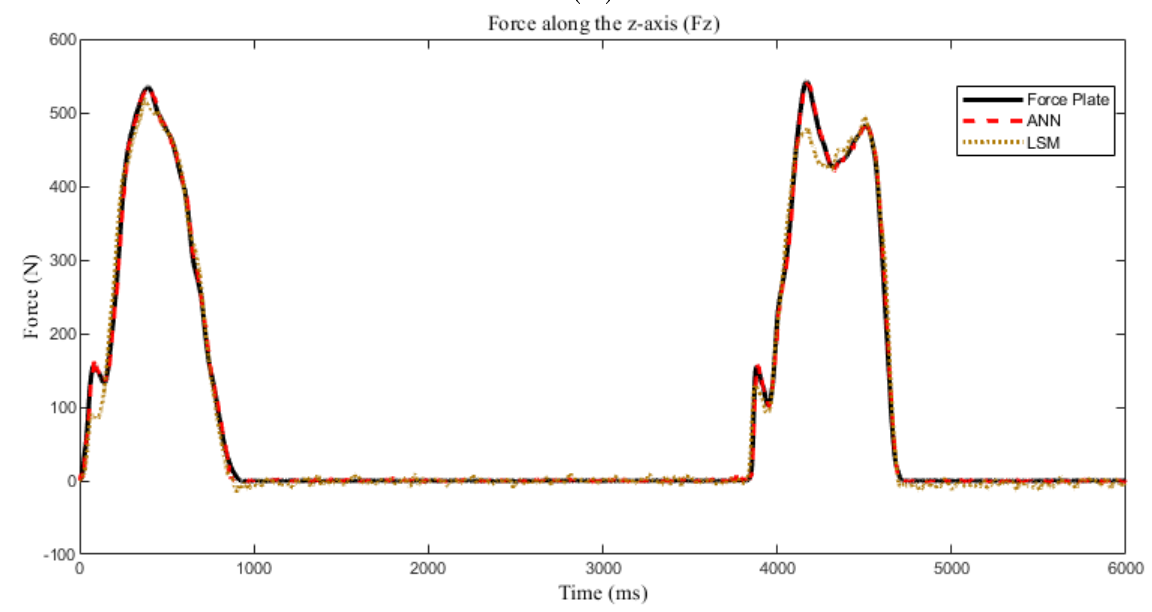

(c)

Figure 4.5: Ground reaction forces of the floor-mounted sensor using the least squares method and a neural network as calibration methods. (a) x-axis force (medio-lateral), (b) y-axis force (anterior-posterior), (c) z-axis force (Normal) 


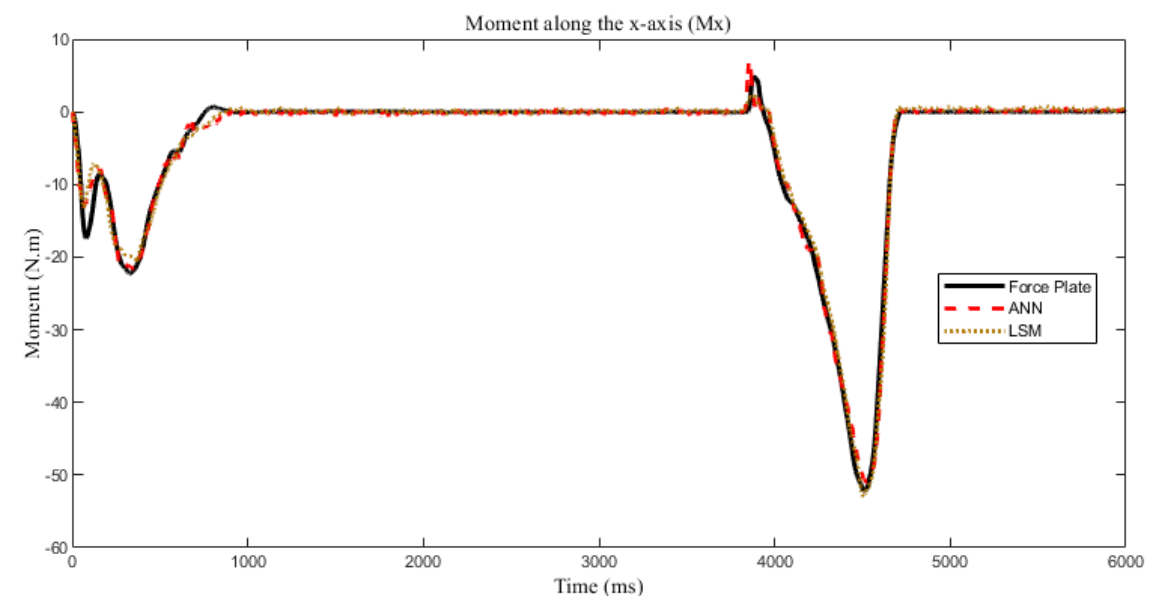

(a)

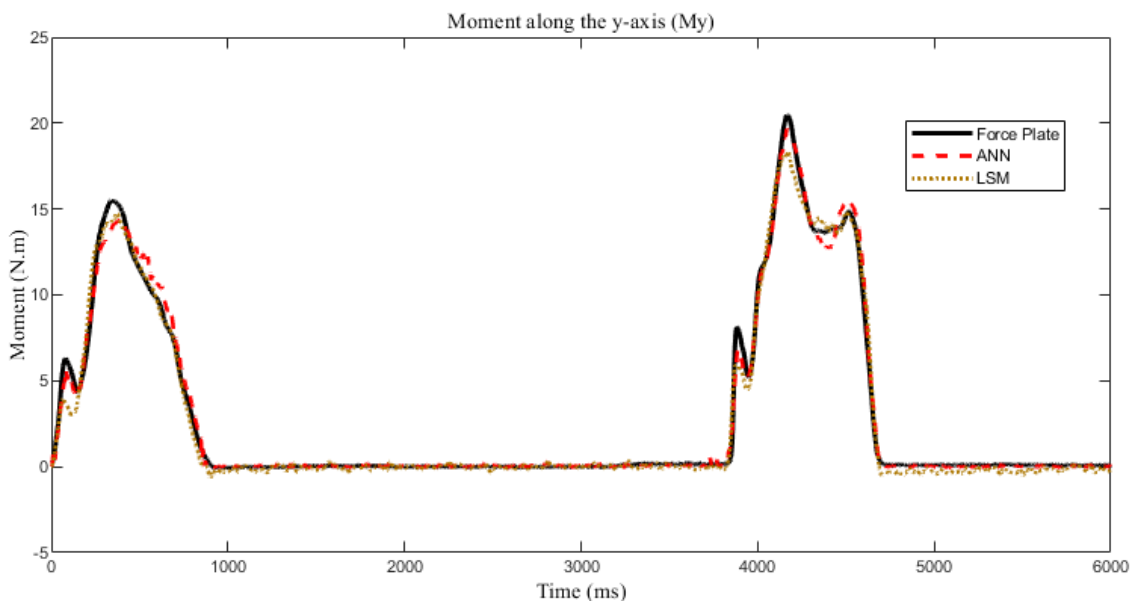

(b)

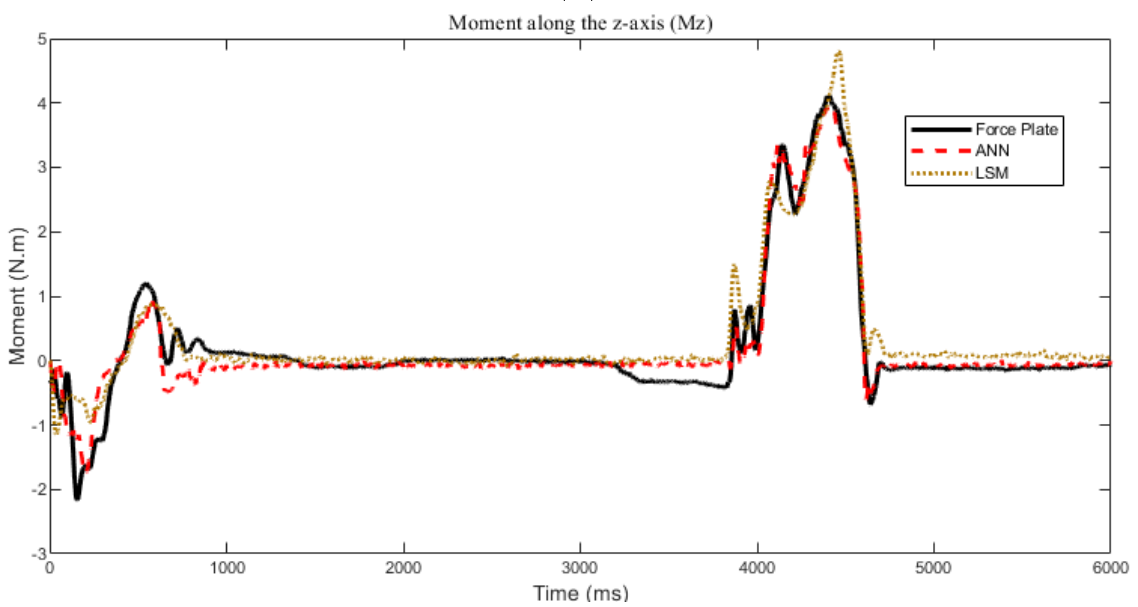

(c)

Figure 4.6: Ground reaction moments obtained from the floor-mounted setting using the least squares approach and a neural network as calibration. (a) x-axis moment, (b) y-axis moment, (c) z-axis moment 


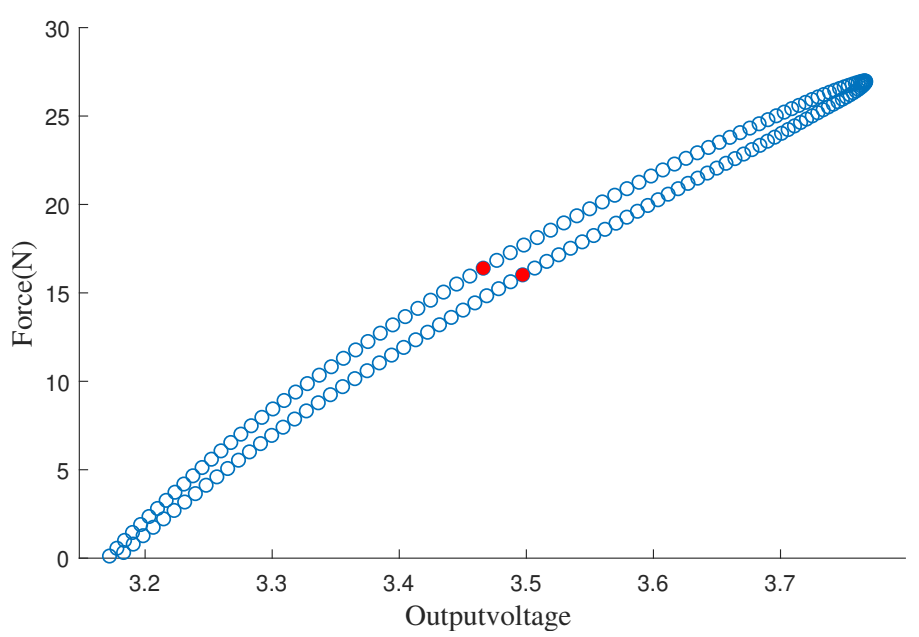

Figure 4.7: Sensor raw voltage and force applied

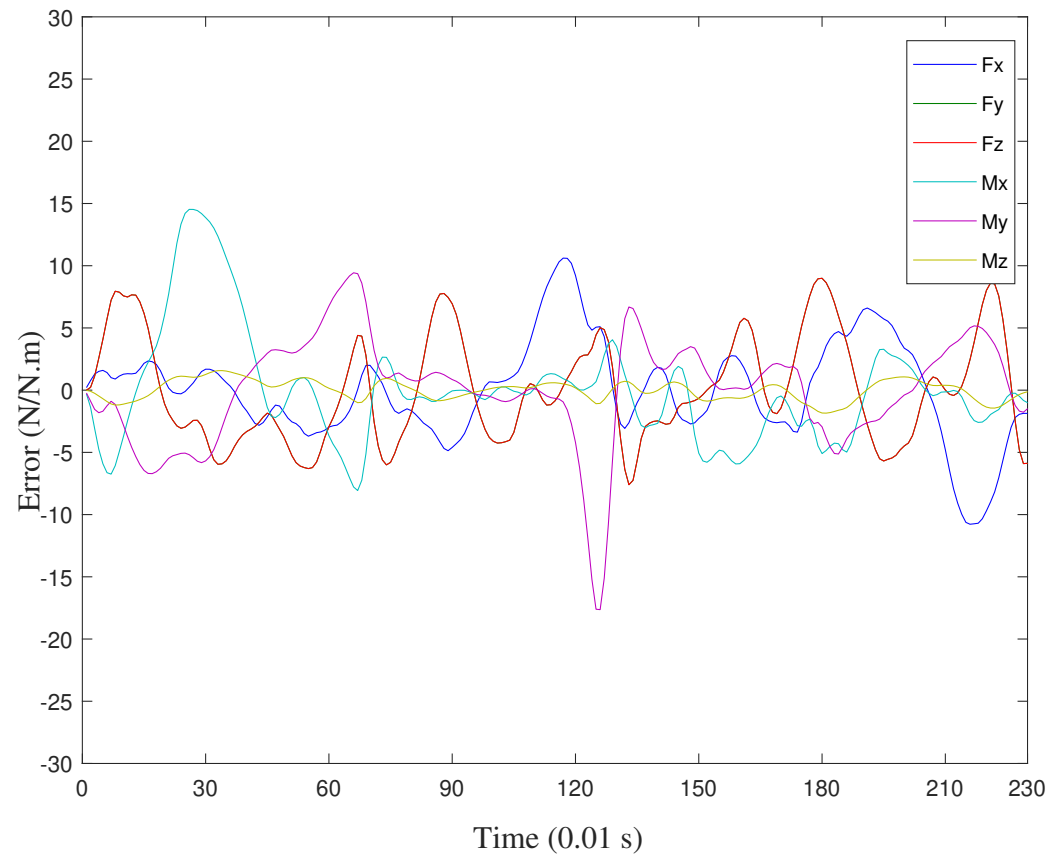

Figure 4.8: Error between six-axis sensor and forceplate for least sqaures method 


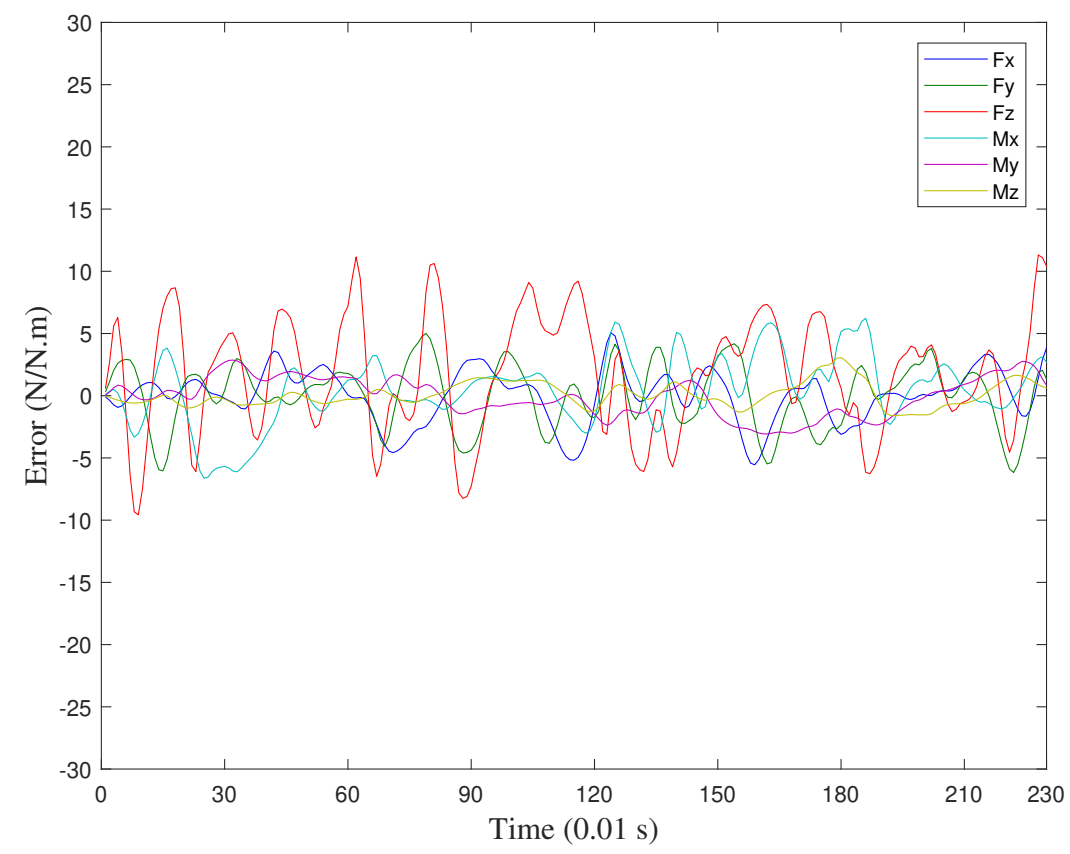

Figure 4.9: Error between six-axis sensor and forceplate for neural network

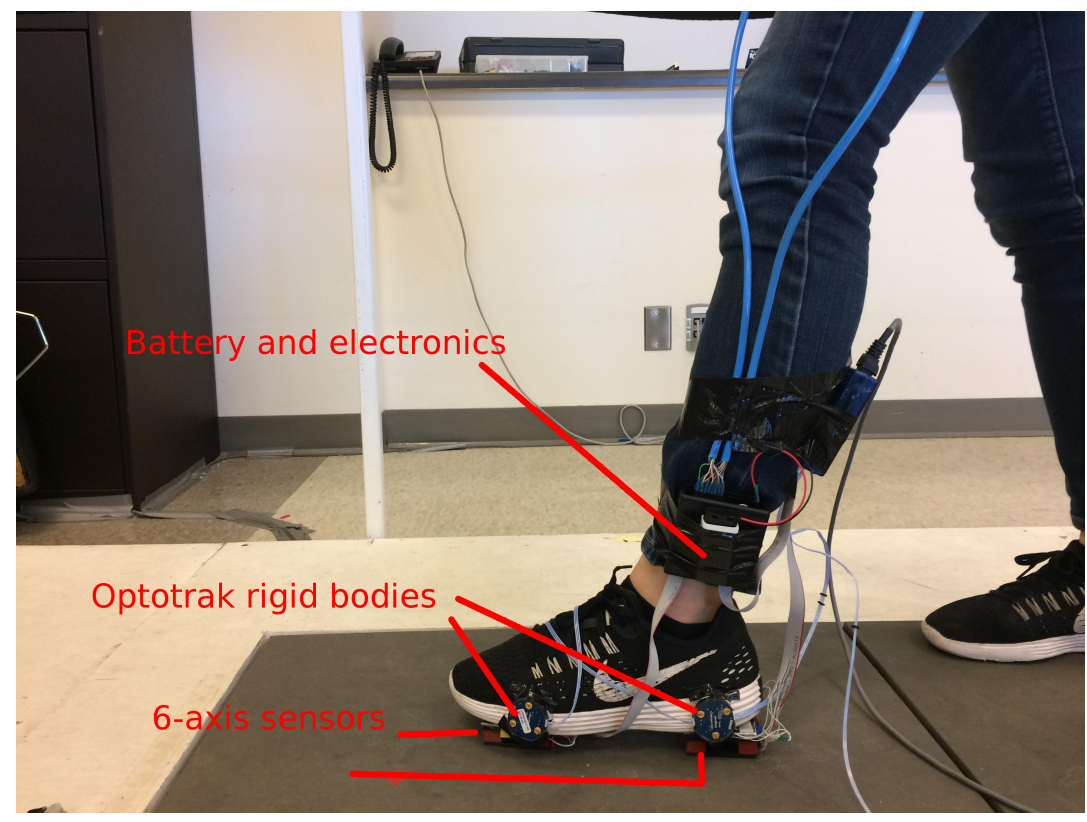

Figure 4.10: Experiment set up for shoe-mounted sensors with two 6-axis sensor systems and 3 Optotrak rigid bodies (one not shown) 
Table 4.2: Comparison of triaxial sensor and six-axis sensor

\begin{tabular}{|c|c|c|c|}
\hline Sensor & X RMSE \% & Y RMSE \% & Z RMSE \% \\
\hline \hline Three axis & $2.30 \%$ & $4.15 \%$ & $1.92 \%$ \\
\hline Six axis & $2.86 \%$ & $2.50 \%$ & $0.84 \%$ \\
\hline
\end{tabular}

to instrument one shoe, and were fixed to the sole of the shoe using tape. One was mounted to the front of the shoe at the toe and one at the heel, allowing for bending at the toe joint during gait. To account for the height difference between shoes caused by the sensors, two wooden blocks of similar sizes to the sensors were taped to the other shoe. This brought each shoe up to a similar height.

When mounted to the shoe, the sensor system and shoe are rotated with respect to the ground during part of the gait cycle. Sensor output is in a different coordinate frame than the force plate data. To account for this, position sensing markers were used to track location and orientation of the sensors. An Northern Digital Optotrak Certus (Waterloo, Canada) was used as a motion capture system to track the position and orientation of rigid bodies attached to shoe and ground.

A total of three Optotrak rigid bodies were used: one attached to the ground, one attached to the shoe sole rear above the heel sensor, and one attached to the shoe sole front above the toe sensor. The instrumented shoe was worn and the participant walked across the platform, stepping on the force plate with the instrumented shoe. The setup can be seen in Figure 4.10. Data for this test was collected at $100 \mathrm{~Hz}$ as it was limited by the speed of the Optotrak Certus. 


\subsubsection{Least squares regression}

A least squares calibration, using a total of more than four steps was performed. Only data during a step was used as the force plate did not capture events when the shoe was not on the force plate. A linear fit achieved reasonably high accuracy for all axes, with an RMS error percent of $9.39 \%$. Detailed statistics for each axis can be found in Table 4.3. These results are less accurate than the floor mounted case, but still produce a satisfactory force and moment estimation.

\subsubsection{Neural network regression}

A nonlinear calibration was used to improve the previous fit. A neural network is used to show that a nonlinear fit can improve the calibration accuracy. The results can be seen in Figure 4.11 and Figure 4.12.

A neural network was trained using the same data set of 233 data points with a $70 \%$ training, $15 \%$ validation, and $15 \%$ testing set split. The network used the same parameters as the floor mounted case and was able to achieve very high accuracy, with detailed results found in Table 4.4. The RMS error was found to be $5.21 \%$. A linear fit can get a good estimate of the ground reaction force and moment, while a nonlinear fit such as the neural net can better capture the relationship between the sensor and forces and moments.

\subsubsection{Angle effects}

To account for the changing angle of the sensors, the rotations of the foot sensors relative to the ground were included as an input in a second neural network. The 
Table 4.3: The experimental results of 6-axis sensor attached to shoe during gait (applied force)

\begin{tabular}{|l|c|c|c|}
\hline \multicolumn{4}{|c|}{ X-Axis Force } \\
\hline Model Type & R-squared \% & RMSE & RMSE \% \\
\hline Linear & $92 \%$ & $3.96 \mathrm{~N}$ & $9.17 \%$ \\
\hline Neural Network & $97 \%$ & $2.12 \mathrm{~N}$ & $4.92 \%$ \\
\hline Neural Network (Optotrak) & $98 \%$ & $1.94 \mathrm{~N}$ & $4.51 \%$ \\
\hline \hline \multicolumn{3}{|c|}{ Y-Axis Force } \\
\hline Model Type & R-squared \% & RMSE & RMSE \% \\
\hline Linear & $98 \%$ & $4.23 \mathrm{~N}$ & $6.65 \%$ \\
\hline Neural Network & $99 \%$ & $2.51 \mathrm{~N}$ & $3.95 \%$ \\
\hline Neural Network (Optotrak) & $99 \%$ & $1.79 \mathrm{~N}$ & $2.82 \%$ \\
\hline \hline \multicolumn{3}{|c|}{ Z-Axis Force } \\
\hline Model Type & R-squared \% & RMSE & RMSE \% \\
\hline Linear & $86 \%$ & $59.28 \mathrm{~N}$ & $11.49 \%$ \\
\hline Neural Network & $99 \%$ & $5.08 \mathrm{~N}$ & $0.98 \%$ \\
\hline Neural Network (Optotrak) & $99 \%$ & $3.84 \mathrm{~N}$ & $0.74 \%$ \\
\hline \hline
\end{tabular}

architecture of this network was the same as previous except with an additional 6 input neurons for each sensor's rotations. This brings the total of input neurons up to 18.

The neural network that used the sensor angles as an input was able to better estimate the forces and moments. The new neural network was able to achieve an RMS error percent of $3.25 \%$, a large improvement when compared to the calibrations without angle input (RMS error percent of $5.21 \%$ ). 


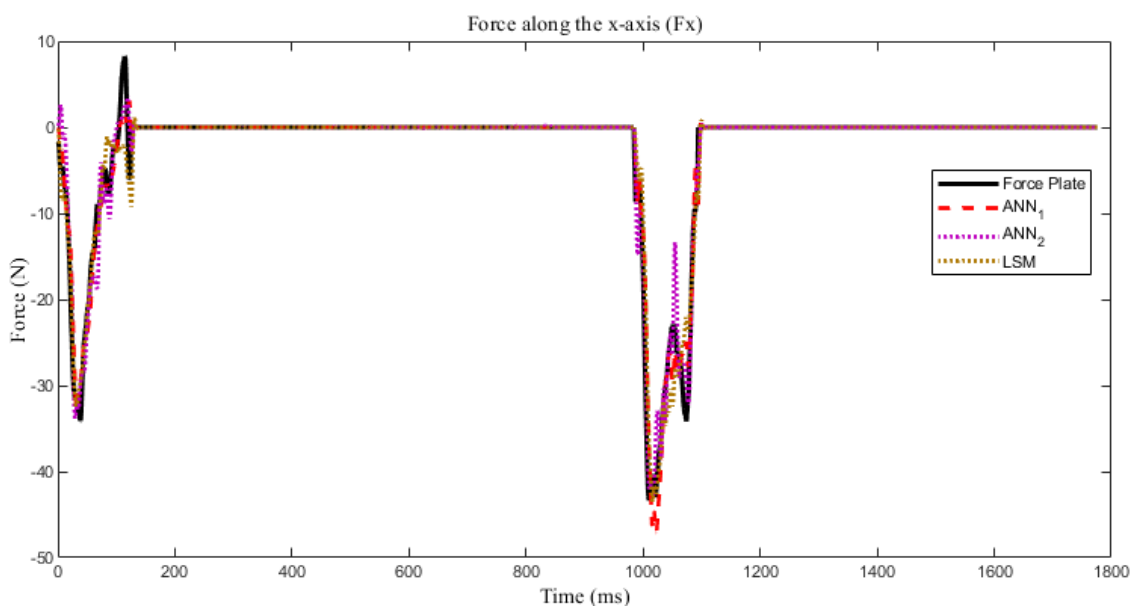

(a)

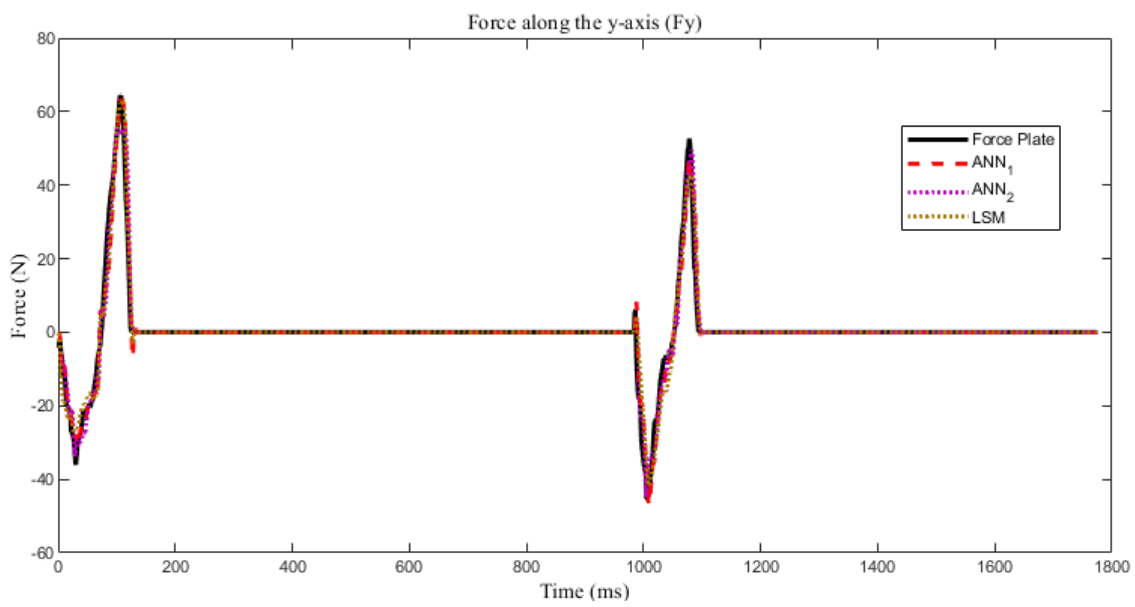

(b)

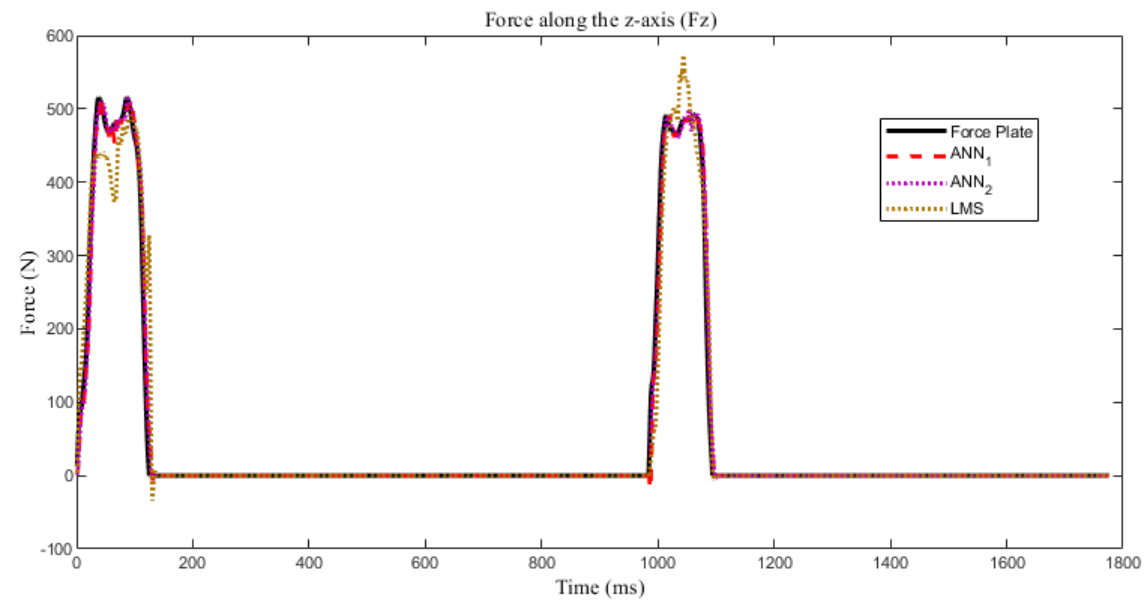

(c)

Figure 4.11: Ground reaction forces obtained from the shoe-mounted sensor using the least squares approach and a neural network as calibration. (a) x-axis force (medio-lateral), (b) y-axis force (anterior-posterior), (c) z-axis force (vertical) 


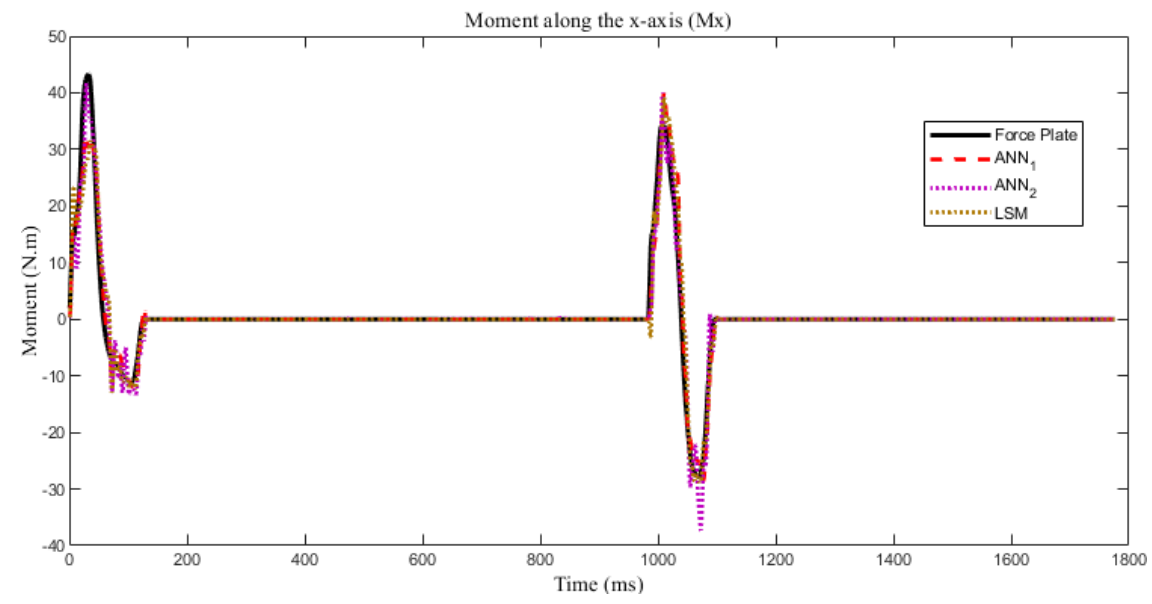

(a)

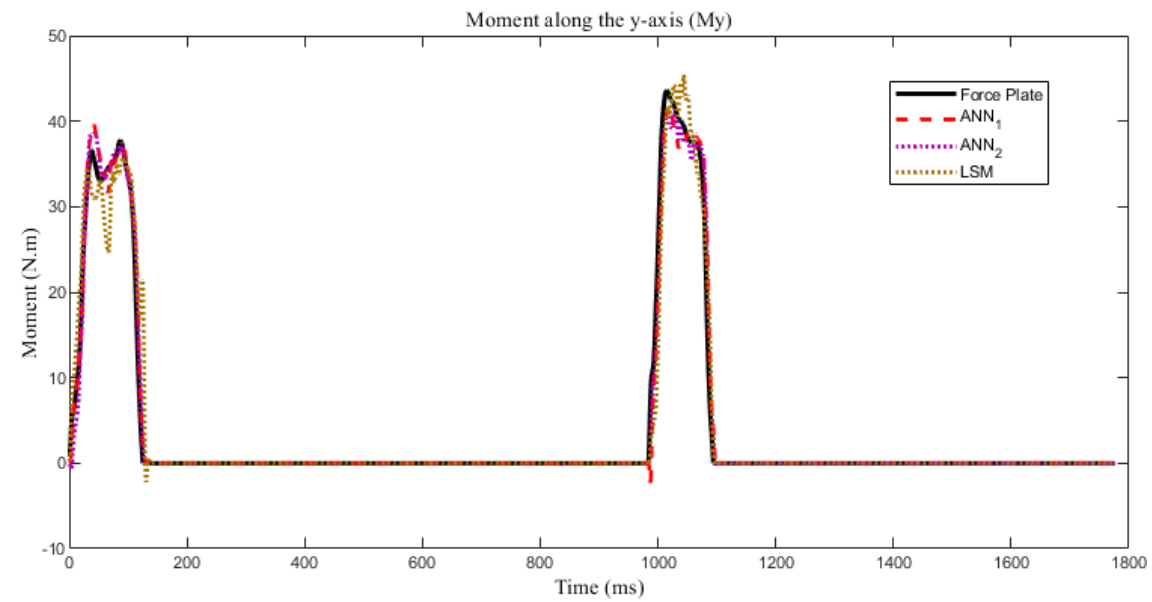

(b)

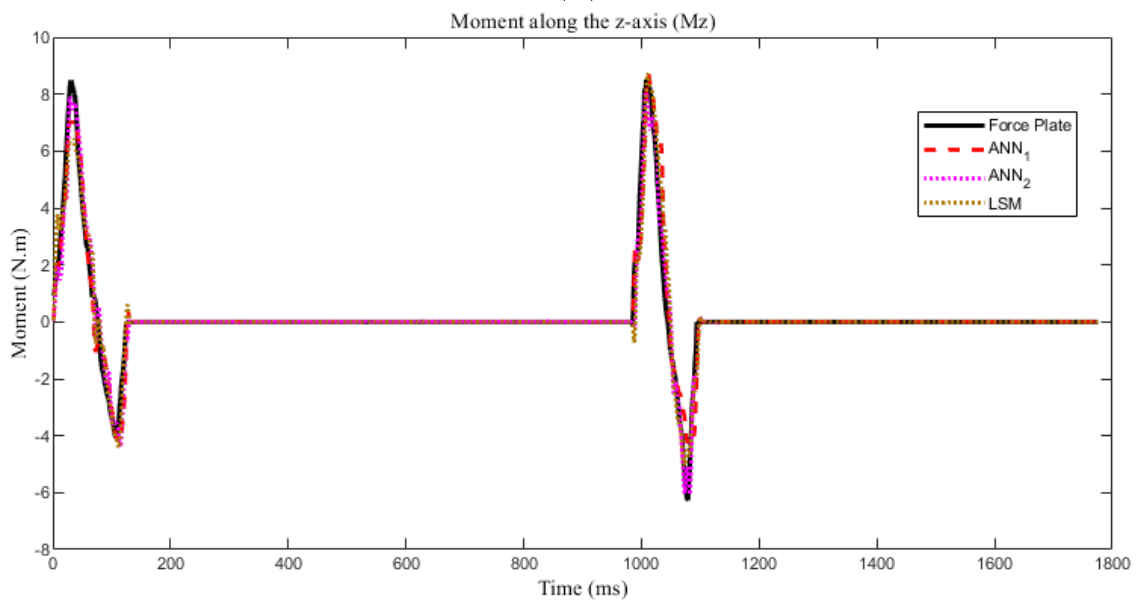

(c)

Figure 4.12: Ground reaction moments obtained from the shoe-mounted sensor using the least squares approach and a neural network as calibration. (a) x-axis force (medio-lateral), (b) y-axis force (anterior-posterior), (c) z-axis force (vertical) 
Table 4.4: The experimental results of 6-axis sensor attached to shoe during gait (applied moment)

\begin{tabular}{|l|c|c|c|}
\hline \multicolumn{4}{|c|}{ X-Axis Moment } \\
\hline Model Type & R-squared $\%$ & RMSE & RMSE $\%$ \\
\hline Linear & $95 \%$ & $4.40 \mathrm{~N} \cdot \mathrm{m}$ & $10.16 \%$ \\
\hline Neural Network & $98 \%$ & $2.73 \mathrm{~N} \cdot \mathrm{m}$ & $6.29 \%$ \\
\hline Neural Network (Optotrak) & $99 \%$ & $1.85 \mathrm{~N} \cdot \mathrm{m}$ & $4.28 \%$ \\
\hline \hline \multicolumn{3}{|c|}{ Y-Axis Moment } \\
\hline Model Type & R-squared $\%$ & RMSE & RMSE \% \\
\hline Linear & $88 \%$ & $4.20 \mathrm{~N} \cdot \mathrm{m}$ & $9.63 \%$ \\
\hline Neural Network & $98 \%$ & $1.53 \mathrm{~N} \cdot \mathrm{m}$ & $3.52 \%$ \\
\hline Neural Network (Optotrak) & $99 \%$ & $1.23 \mathrm{~N} \cdot \mathrm{m}$ & $2.82 \%$ \\
\hline \hline \multicolumn{3}{|c|}{ Z-Axis Moment } \\
\hline Model Type & R-squared $\%$ & RMSE & RMSE \% \\
\hline Linear & $96 \%$ & $0.77 \mathrm{~N} \cdot \mathrm{m}$ & $9.21 \%$ \\
\hline Neural Network & $94 \%$ & $0.96 \mathrm{~N} \cdot \mathrm{m}$ & $11.60 \%$ \\
\hline Neural Network (Optotrak) & $99 \%$ & $0.36 \mathrm{~N} \cdot \mathrm{m}$ & $4.35 \%$ \\
\hline
\end{tabular}

\subsection{Conclusion}

The new sensor system combines fiber optic based and FlexiForce sensors to create a wearable, six-axis sensor for gait. It is lightweight and compliant which helps maintain natural gait. The sensor was validated to have accurate performance in a floor-mounted configuration. Accurate results are obtainable using a linear calibration, achieving a mean RMS error percent of $4.49 \%$. A nonlinear fit is able to capture nonlinearities in the rubber and LTVs; a neural network was used as an example of a nonlinear fit. It is able to achieve a higher accuracy than the linear fit, reaching a mean RMS error of 
$2.68 \%$.

As a wearable sensor, the linear calibration is able to achieve a good force and moment estimation. The mean RMS error for the linear calibration was found to be 9.39\%. A neural network is able to achieve higher accuracy than a linear calibration, with a mean RMS error of $5.21 \%$. Sensor orientation was included as an input on a second neural network to attempt to improve this result. This neural network achieved a mean RMS error percent of $3.25 \%$. Including the orientation of the sensors helps to improve accuracy.

The final sensor system is lightweight and wearable, allowing for measurements to be taken outside of a laboratory setting. It is capable of measuring the complete ground reaction force and moments for gait analysis applications and centre of pressure measurement for fall prevention applications. 


\title{
Chapter 5
}

\section{Design, Development and Calibration of a Lightweight, Compliant 6-Axis Optical Force/Torque Sensor}

\begin{abstract}
This chapter introduces the fabrication of a six degree-of-freedom force and torque sensor based on fiber-optic sensing technology and its novel calibration methodology. The sensor is cost-effective, lightweight and flexible with a large force and torque measurement range suitable for biomechanics and rehabilitation systems particularly when a wearable sensing system is desired. Six fiber-optic sensing elements are used to detect three main forces $F x, F y, F z$ and three main torques $T x, T y, T z$. Sensor data was collected by applying dynamic forces and torques with various magnitudes, directions and frequencies and compared with measurements obtained from a standard force
\end{abstract}


and torque reference. The proposed calibration procedure is intended to reduce errors stemmed from a nonlinear force-deformation relationship and to increase the estimation speed by splitting the calibration into two estimation models: a linear model, based on a standard Least Squares Method $(L S M)$ to estimate the linear portion; and a nonlinear Decision Trees model $(D T)$ to estimate the residuals. Both models work simultaneously as a single calibration system named Least Squares Decision Trees $L S D T$. Using $L S D T$, the estimation speed increased by $55.17 \%$ and the Root Mean Square Errors (RMSE) reduced to $0.53 \%$. In comparison, each model separately had a root mean square errors of $1.26 \%$ and $4.70 \%$ for $D T$ and $L S M$, respectively.

\subsection{Introduction}

Electronic equipment responsive to external perturbations and mechanical deformations have attracted significant attention in several applications such as biomechanics, Human Machine Interaction (HMI) and rehabilitation systems. Providing a precise measurement of force and torque is essential to effectively address the interaction between human body and external environment and to monitor the body motion and balance through wearable sensing devices. Six-axis force and torque sensors are the optimum solution for such applications due to their strong ability to measure the complete degree of freedom of any force or torque applied at a certain point.

Although these six-axis force and torque sensors are important and have great measurement capabilities, they are complex in their manufacturing and assembly process. The bonding procedure of sensing elements, for example, requires high precision, making it time consuming and therefore costly in terms of time and finance 
since the sensing systems depend on strain gauges as their sensing technology [189-192]. To obtain a satisfactory sensitivity for 6 -axis, force and torque strain gauge sensors, researchers incorporate large numbers of sensing elements (strain gauges) distributed along the body undergoing deformation [193-196]. This can cause communication bandwidth problems since a large data transmition between strain gauges and the main microcontroller is required which can lead to unwanted lengthy delay in data transfer and a higher power consumption [197]. Even though 24 strain gauges were bonded along the elastic body in [198-200], the measurement range was not satisfactory for these sensors made of rigid metals. $F z$ for instant, was $500 \mathrm{~N}$ in [199].

As many as 32 strain gauges were cemented on the body structure of sensors in [159, 196, 201], resulting in larger amounts of data being transmitted. In an attempt to reduce the online communication bandwidth and number of wires out of the sensor, researchers used electronic components such as an internal microcontroller to group the sensing elements and pre-process data locally inside the sensor unit. In doing so, only the relevant six axes force and torque raw data through only six wires to a data acquisition system is transferred for the final processing [199]. However, this approach does not provide any solutions to decrease the amount of transferred data as it only facilitates data transmission to the main microcontroller. The rigidity, bulkiness and weight concerns involved in the development of strain gauge-based force and torque sensors make them less suitable for biomechanics and rehabilitation applications where compliance is key. Two strain gauge-based force and torque sensors (Mini45 load cells, from ATI Industrial Automation, Inc.) were mounted underneath a shoe to measure the total ground reaction force and estimate the center of pressure [202, 203]. Although the sensors were able to accurately measure force and moments, they were rigid since these sensors were made of metals that can cause discomfort for the wearer. Furthermore, 
extra rigid metal plates were used, one mounted on the top of both sensors along the outsole and two underneath each load cell [203] adding extra weights to the sensor which can easily influence natural human movements and cause fatigue.

Capacitive-based transducers are also broadly used in force and torque measurement systems [204-208]. Although these capacitive-based transducers are adequate in precision and have wide force and torque measurement range, they are also highly susceptible to electromagnetic interference and may experience drift and small spatial resolution.

A piezoresistive-based six-axis force and torque sensor prototype was developed in $[158,209,210]$. These sensors exhibited a relatively good sensitivity but have noticeable linearity errors and low accuracy and small force measurement range. Other drawbacks include considerably high stiffness, low breaking force and temperature compensator for each individual sensing element [64]. Piezoelectric-based sensors are also widely used in force and torque measurement systems [211, 212]. Electric polymer foils are the most extensively used material in biomedical applications such as polyvinylidene fluoride (PVDF) and cellulose nanofibril flexible piezoelectric-based polymer foil multi-axis force sensors [213, 214]. Since the proposed material is highly sensitive to pyroelectricity (the material generates a voltage when there is a change in temperature) [70], a temperature compensator is necessary. In addition, the sensors were capable of measuring forces but not torques, and the materials were structurally fragile and prone to damage [71].

Fiber-optic sensing technology is another attractive technique used to measure force and torques; particularly FBG-based sensors [215] with their lightweight, small size, good accuracy, high longitudinal strain resolution with no sensitivity to electromagnetic 
interference, and negligible hysteresis effects [216]. However, their high sensitivity to small temperature variation and their high cost of signal processing reduce the versatility of their use in biomechanical measurements.

Calibration is critical for multi-dimensional force and torque transducers. It requires complex data processing and multi-step sensor characterization due to cross coupling effect and complicated nonlinearities. Conventional calibration methods such as least squares (calibration matrix) has been extensively used for calibration of multi-axis $\mathrm{F} / \mathrm{T}$ sensors $[217,218]$. Although calibration matrix is relatively effective in minimizing crosstalk effects, the relationship between input and output is assumed to be linear.

Compliant sensors exhibits nonlinear behavior due to large deformation generated by the applied $\mathrm{F} / \mathrm{T}$ which results in nonlinear output, therefore a certain portion of sensor data cannot be fully characterized with the calibration matrix. Calibration method based on finite element analysis (FEA) simulation was used in [219, 220]. This method is also based on several assumptions such as isotropic elasticity, negligible deformation in cross beams, and strain gauges being bonded and aligned with high precision which cannot be experimentally attained. A non-parametric approach such as Artificial Neural Networks (ANN) has the ability to predict any nonlinear relationship and has been applied to calibrate multi-axis $\mathrm{F} / \mathrm{T}$ transducers [221-223]. However, ANN has a few shortcomings: extensive network scale, considerable amount of training time and large data sets are needed for training.

The presented 6-axis fiber-optic force/torque sensor with its novel calibration methodology is flexible, low noise, extremely lightweight, cost effective and has large force and torque measurements range with high precision. Thus, it is usable in a variety of biomechanics and rehabilitation applications. The novel calibration procedure is able 
to precisely characterize the slight nonlinear behavior generated from force-deflection relationship. This calibration approach mainly relies on a nonlinear estimation model that is used to predict the errors and works simultaneously with a standard linear least squares model, providing a very high-quality sensing performance.

\subsection{Sensor Architecture}

The current 6 -axis $\mathrm{F} / \mathrm{T}$ sensing system shown in Figure 5.1 was built upon a similar fiber-mirror transducer technology based on light intensity modulation we reported in [1]. In this design, multiple optical sensing elements were configurated in a single sensor unit enabling a simultaneous measurement of applied forces and torques along the six normal axes creating a novel compliant 6 -axis $\mathrm{F} / \mathrm{T}$ sensor. The prototype was able to precisely describe the applied forces and torques in real-time.

\subsubsection{Sensor Design and Fabrication}

The exploded view seen in Figure 5.1 illustrates the conceptual design of the proposed 6-axis force and torque sensor. Six optical sensing elements were developed where each element is composed of $0.75 \mathrm{~mm}$ diameter polymethyl methacrylate (PMMA) fibers: one to transmit the light and the other to receive the reflecting light into a light detector. The light transmitting fiber is joined at the tip to a high-intensity, white light emitting diode (OVLEW1CB9, $100 \mathrm{~mW}$ from TT Electronics/Optek Technology,

Inc.) as the light source. In this design, two LEDs are used to feed the six transmitting fibers, resulting in lower power consumption. The receiving fiber is joined at the tip to a light-to-voltage converter (LTV), model TSL257 from AMS Austria Micro Systems 


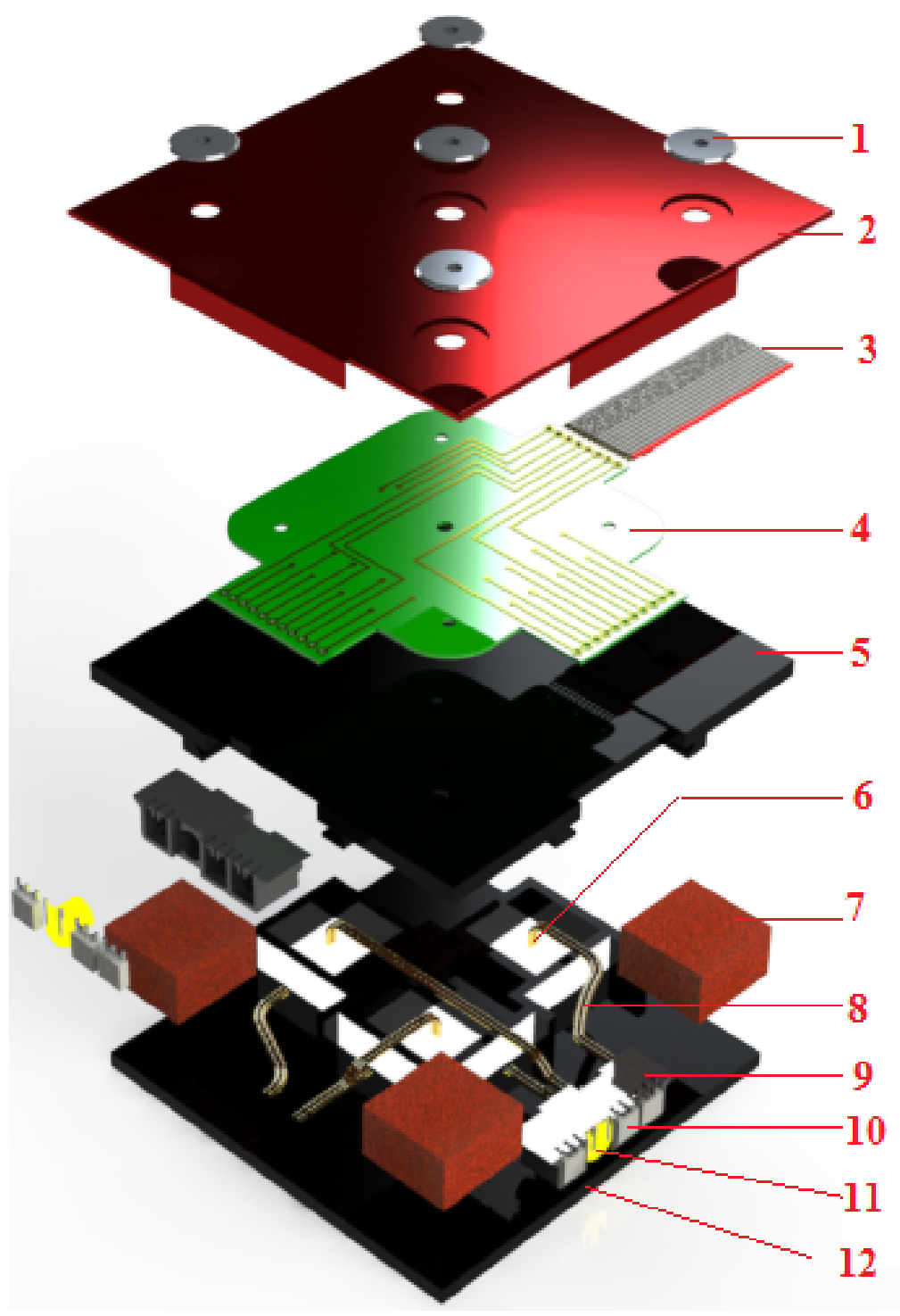

Figure 5.1: Structure of the proposed sensor. (1). Screw (2). Outer shield (3). Flat data cable (4). Printed circuit board for reliable connections (5). Top rigid layer (6). Light reflecting mirror (7). Compliant block (silicon rubber) (8). Optical fiber (9). LEDs and LTVs holder (10). LTV (Model: TSL-275) (11). LED (Model: OVLEW1CB9) (12). Bottom rigid layer.

AG. In an effort to obtain higher sensitivity and increase the light intensity, the LTVs and LEDs were sanded down to minimize the distance between the outside surface of the epoxy case and the semiconductor chip, then polished to create a smooth, flat and 
shiny surface. The fibers are fixed to the top plate of the device along with the LEDs and LTVs while the electrical wires are hooked to the top plate and feed the electrical signals to the processing unit. Here, the electrical wires can move relative to the top plate, whereas the fibers cannot. The fibers exhibit an excellent light transmission and illumination characteristics, and superior flexibility compared to glass optical fibers for creating shapes and orientation at the ends of each sensing element.

The top plate was 3-D printed in rigid black polylactic acid (PLA) with small paths $1.8 \mathrm{~mm}$ in width along the inner surface of the top plate to help guide each pair of fibers to its corresponding reflective surface, LTV and LED, as shown in Figure 5.2. Six reflective surfaces are distributed on the 3D printed bottom plate via black PLA chambers (three open and three enclosed) and six $0.65 \mathrm{~cm}$ by $1.2 \mathrm{~cm}$ mirrors with adhesive backings which are affixed to a wall in each chamber, as depicted in Figure 5.2.

Sensor flexibility is due to the elastic material made of silicon rubber mounted between the top and the bottom plates. Three adhesive mirrors are taped on the surface parallel to the bottom and top plates whereas the other three mirrors are taped perpendicular to the bottom plate. Both groups work simultaneously to reflect six optical signals that are used to describe all forces and torques.

To avoid the optical crosstalk between the axes of the sensor, each optical sensing element is isolated using a 3D printed plastic barrier, thereby encasing each reflective surface and each tip of transmitting and receiving fibers. A $5 \mathrm{~cm}$ by $5 \mathrm{~cm}$ PCB was designed to connect the electronic components of the sensor. Finally, the top plate is used as a shield to protect the electronic components and the printed circuit board. Figure 5.3 shows the assembled sensor. 


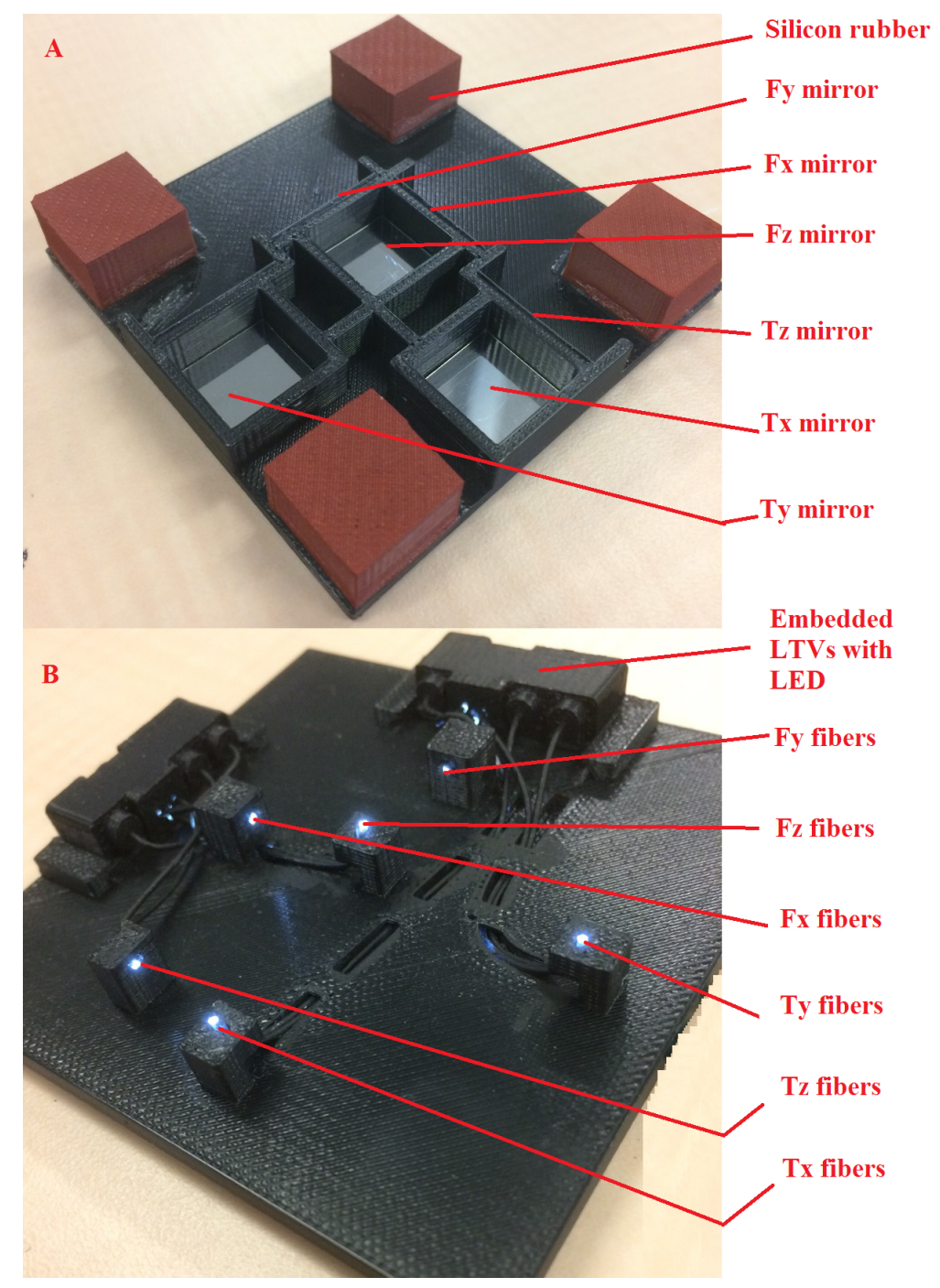

Figure 5.2: Configuration of sensing elements. Each force and torque component has only one sensing element that contains LED, pair of fibers, LTV and reflective surface embedded along the top (A) and bottom (B) layers.

\subsubsection{Principles of sensing}

The proposed sensor has the ability to measure the three main components of force ( Fx, $F y, F z)$ and three main components of torque $(T x, T y, T z)$ using reflective intensity modulation via fiber-optic technology. To measure the applied forces and torques, at least six signals from six various locations and directions along the internal structure 


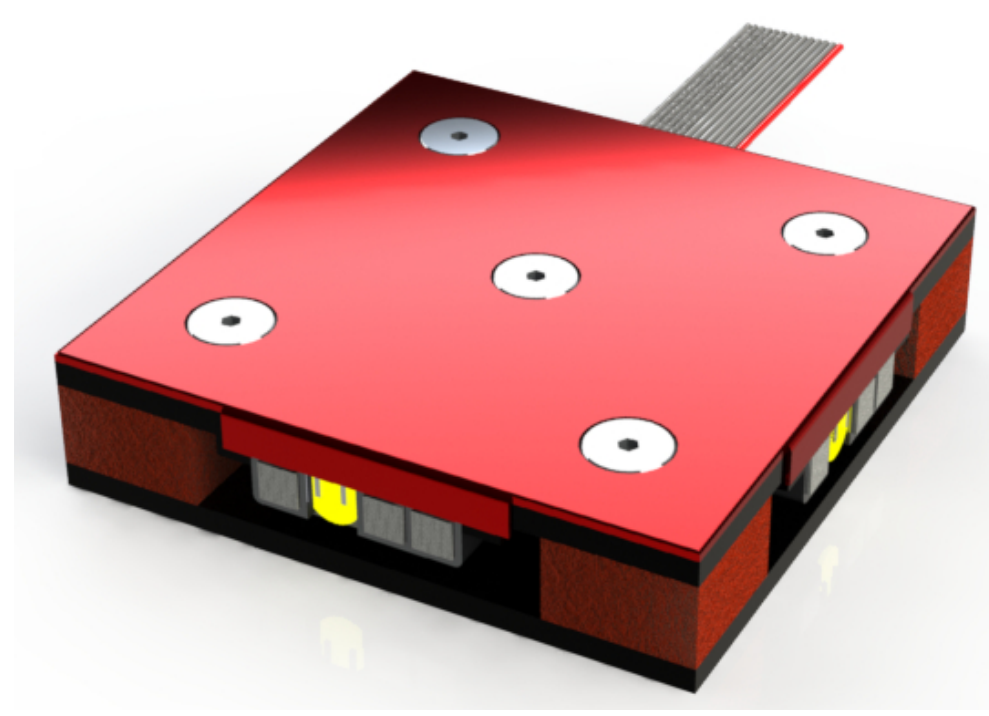

Figure 5.3: Prototype 6-axis fiber-optic $\mathrm{F} / \mathrm{T}$ sensor in its assembled state.

of a sensor are required [224].

Figure 5.2. demonstrates the arrangement of the transmitting and receiving fibers for each axis and their corresponding reflective surfaces which create six fiber-optic sensing elements. Each pair of fibers is positioned perpendicular to its reflective surface, enabling the continuous measurement of light intensity of the reflected optical signal. The sensing elements are configured on the plate to capture the six main forces and torques of the top plate relative to the bottom plate. Three reflective planes are situated parallel to the bottom layer. The reflective surface allocated to measure the vertical force $F z$ is affixed at the center of the bottom plate while the other two reflectors are installed on both sides of the adjoining open chambers. To capture torque, one reflector is mounted along the x-axis from the $F z$ reflector, which is dedicated for the torque detection around the y-axis $(T y)$ while another reflector is mounted on the other side along the y-axis and is designated to reflect the light transmitted from the torque sensor Tx; as shown in Figure 5.4. The other end of the fibers is connected to an 


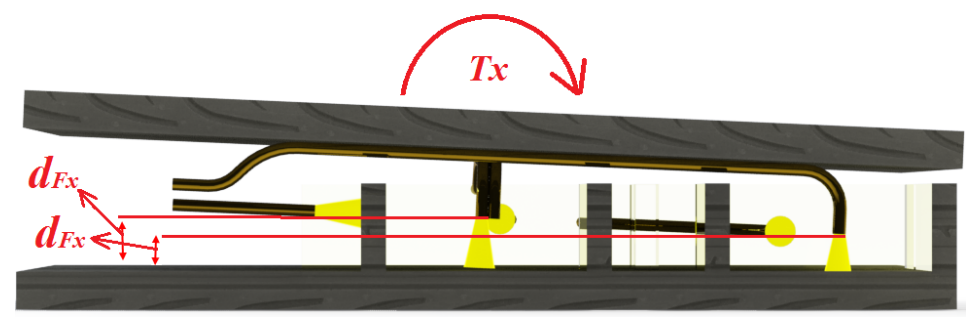

Figure 5.4: Sensor behavior due to applied torque around the $\mathrm{x}$-direction. The top plate tilts which generates light intensity variation enabling $T x$ measurement.

optical source (LED) and a Light-to-Voltage convertor (LTV).

The distance between optical sensing elements and reflectors is fixed to $3.5 \mathrm{~mm}$ using four $1 \mathrm{~cm}$ tall silicon rubber blocks, which remains constant when there is no external perturbation acting on the sensor. The value of measured light intensity stays the same, indicating the absence of force and torque. The intensity of the reflected light changes substantially based on the relative distance variation induced by external force or torque. The distance between the fiber tips and the reflectors $F z, T x, T y$ are termed $d_{F_{z}}, d_{T_{x}}$ and $d_{T_{y}}$ respectively. The force information can be obtained directly by observing the change in light intensity resulting from the change in distance $d_{F z}$ when a vertical force along the z-axis is applied. The distances $d_{T_{x}}$ and $d_{T_{y}}$ also change, thus the light intensity variations produced by $T x$ sensor is a function of the vertical force $F z$ and torque $T y$. Once the vertical force $F z$ is obtained (known), $T x$ can be recovered. Similarly, the intensity variations generated by $T y$ sensor is a function of both vertical force $F z$ and torque applied about the x-axis $T x$. Ty is recovered from its allocated sensing element.

When an external torque is applied about the x-axis, the structure of the sensor deforms and the top plate of the sensor rotates with respect to the bottom plate leading to a decrease in the distance $d_{T_{x}}$ (the light intensity of $T x$ sensor increases) and there is 


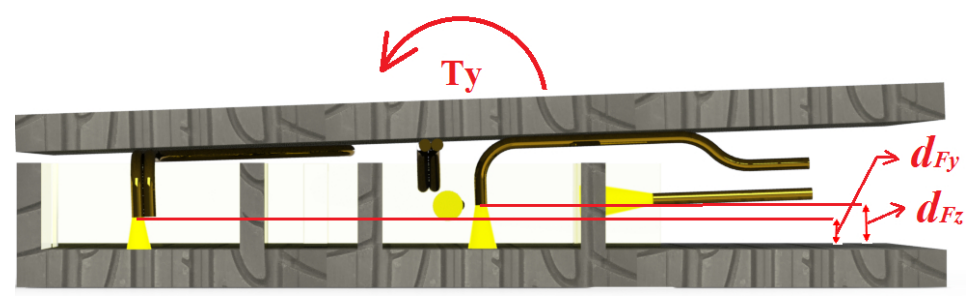

Figure 5.5: Sensing principle of the torque applied around the y-direction. The distance between the fibers tip and their respective reflecting mirror changes. This decreases or increases the intensity of detected light using LTVs.

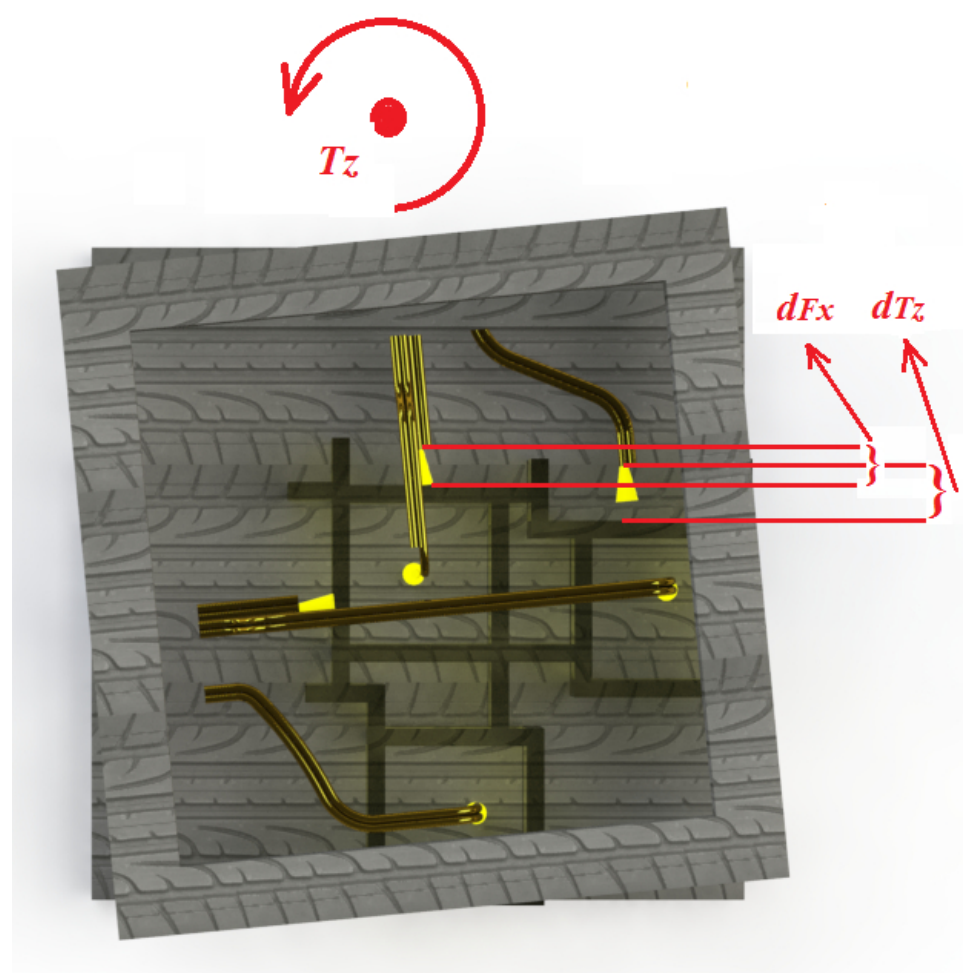

Figure 5.6: Torque $T z$ sensing mechanism. The top plate rotates with respect to the bottom plate and the torque is measured in accordance to the produced light intensity alteration.

a slight increase in the distance $d_{F_{z}}$ as can be seen in Figure 5.4. The torque $d_{T_{x}}$ is then determined by calculating the difference between the two generated intensities. In the same way the torque applied about the y-axis $d_{T_{y}}$ is obtained as depicted in Figure 5.5. These three main sensing points and their reflectors are affixed on the bottom plate and are mainly employed to measure normal force $F z$ and torques $T x$ and $T y$. 
The other three sensors are used to measure the two components of shear forces: $F x$ and $F y$ as well as the torque about the z-axis $T z$ so that each reflector of these sensors is mounted perpendicular to the bottom layer. The fiber-optic tips move forward and backward in parallel to the bottom plate. When a longitudinal shear force is applied, the top plate moves with respect to the bottom plate, and the distances $d_{F_{x}}, d_{F_{y}}$ and $d_{T_{z}}$ changes, leading to an increase or decrease in light intensity detected by their respective LTVs. Light intensity changes in the $F x$ sensing element is only a function of longitudinal force in the x-direction. This also applies when there is a force in the y-direction, Fy. However, the variation of light intensity produced by $T z$ sensor is a function of both $F x$ and $T z$. Once the force in the x-direction is obtained, the torque $T z$ can be determined. When a shear force is applied in the x-direction the difference between the reflected light intensities of $T z$ and $F x$ remain constant since their distances $d_{F_{x}}$ and $d_{T_{z}}$ are equal regardless of the amount of force.

When the top plate of the sensor rotates about the z-axis, $d_{F_{x}}$ and $d_{T_{z}}$ are no longer equal, therefore the difference between the two generated intensities is used to determine $T z$ as shown in Figure 5.6.

\subsection{Sensor Characterization}

The intended sensor applications are mostly incorporating dynamic locomotion with considerable displacement and strain, including gait analysis and total ground reaction force/moment measurements during walking, running, as well as human-machine interactions. Compliant sensors typically exhibit nonlinear behaviours caused by the mass and viscoelastic properties of the flexible materials. In the current sensor, there are elastic materials used between the rigid top and bottom plates, therefore it is 
required for such class of sensors to compensate for the nonlinear behaviours induced by large strains.

A new calibration procedure is proposed and applied to better characterize the developed 6-axis $\mathrm{F} / \mathrm{T}$ sensor. The methodology has the ability to reduce errors, obtaining a higher accuracy of force and torque measurements. In this approach a linear and a nonlinear estimation model are used together as a single characterization system named Least Squares Decision Trees (LSDT) estimation model to achieve the best results.

\subsubsection{Experimental setup}

Data obtained from the proposed optical sensor was compared to force measurements acquired from a standard 6-axis force and torque sensor from Industrial Automation (ATI Delta SI-660-60). As shown in Figure 5.7, the developed sensor was mounted to the reference while forces and torques are applied along the six normal axes. Voltage signals from both sensors were simultaneously acquired through the same data acquisition system, NI PCI 6229 DAQ, from National Instrument at a sampling rate of $1 \mathrm{kHz}$ using twelve channels: six channels to connect the prototype and another six channels to connect the reference. Voltages are received and processed by MATLAB using xPC-Target real-time environment. Although the intended application of this sensor is gait analysis, different force inputs where applied to the sensor to evaluate the sensing performance in addition to the gait experiments. 


\subsubsection{Linear Estimation via Least Squares Method (LSM)}

Multiple linear least squares regressions were performed as a first calibration methodology to pre-characterize the raw data (output voltage) and obtain a linear force and torque estimator. For each axis, a large data set was collected as analog signals to be used in calibration process. A linear Least Squares Method $(L S M)$ is applied to define the following 42 calibration parameters which include gain matrix $A_{n, m}$ where $n=m=6$, and zero-offset matrix $w_{n, m}$, where $n=1$ and $m=6$. $u_{n}$ represents the output voltages from the developed sensor. The linear estimation model is given by:

$$
\left[\begin{array}{c}
\hat{F}_{x} \\
\hat{F}_{y} \\
\hat{F}_{z} \\
\hat{T}_{x} \\
\hat{T}_{y} \\
\hat{T}_{z}
\end{array}\right]=\left[\begin{array}{cccccc}
a_{1,1} & a_{1,2} & a_{1,3} & a_{1,4} & a_{1,5} & a_{1,6} \\
a_{2,1} & a_{2,2} & a_{2,3} & a_{2,4} & a_{2,5} & a_{2,6} \\
a_{3,1} & a_{3,2} & a_{3,3} & a_{3,4} & a_{3,5} & a_{3,6} \\
a_{4,1} & a_{4,2} & a_{4,3} & a_{4,4} & a_{4,5} & a_{4,6} \\
a_{5,1} & a_{5,2} & a_{5,3} & a_{5,4} & a_{5,5} & a_{5,6} \\
a_{6,1} & a_{6,2} & a_{6,3} & a_{6,4} & a_{6,5} & a_{6,6}
\end{array}\right]\left[\begin{array}{l}
u_{1} \\
u_{2} \\
u_{3} \\
u_{4} \\
u_{5} \\
u_{6}
\end{array}\right]+\left[\begin{array}{l}
w_{1} \\
w_{2} \\
w_{3} \\
w_{4} \\
w_{5} \\
w_{6}
\end{array}\right]
$$

the model parameters values are determined as follow :

$$
A=\left[\begin{array}{rrrrrr}
2.02 & -0.16 & -0.47 & -0.01 & 0.10 & -0.00 \\
-0.05 & 0.88 & 0.17 & -0.03 & -0.00 & 0.00 \\
0.45 & -0.08 & 2.58 & -0.01 & 0.05 & 0.00 \\
-0.02 & -0.05 & 3.29 & 0.10 & 0.03 & -0.00 \\
-0.42 & 0.24 & -0.44 & -0.04 & -0.08 & -0.00 \\
0.36 & -1.51 & -2.32 & 0.04 & -0.11 & -0.06
\end{array}\right] \times 10^{-2}
$$




$$
w=\left[\begin{array}{r}
-2.18 \\
-0.30 \\
-3.36 \\
0.01 \\
-0.08 \\
0.01
\end{array}\right] \times 10^{-2}
$$

The least squares estimator of the normal force acting along the z-axis $\hat{F}_{z}$, produces a Root Mean Square Error (RMSE) of $17.21 \mathrm{~N}$ out of a force measurement range of $1039.26 \mathrm{~N}$. The normalized RMSE is $1.65 \%$. The coefficient of determination or goodness of fit, R-squared $R^{2}$, was found to be $99 \%$, confirming a precise estimated normal force.

The RMSE, normalized RMSE and $R^{2}$ were calculated as follows:

$$
\begin{gathered}
R M S E=\left[\frac{1}{n} \sum_{i=1}^{n}\left(F z_{i}-\hat{F} z_{i}\right)\right]^{\frac{1}{2}} \\
\text { Normalized } R M S E=\frac{R M S E}{\text { Force } / \text { Torque Range }} \times 100 \% \\
R^{2} \%=\left[1-\frac{\sum_{i=1}^{n}\left(F z_{i}-\hat{F} z_{i}\right)^{2}}{\sum_{i=1}^{n}\left(F z_{i}-\bar{F} z_{i}\right)^{2}}\right] \times 100 \%
\end{gathered}
$$

For the forces acted along the x-axis, the shear force estimator $\hat{F}_{x}$ generates an RMSE value of $10.02 \mathrm{~N}$. The corresponding normalized RSME is $7.72 \%$ with $R^{2}$ of $97 \%$. The shear force estimator $\hat{F}_{y}$, generates an RMSE value of $5.44 \mathrm{~N}$, normalized RSME of $5.161 \%$, and $R^{2}$ of $93 \%$. For torques applied about the $\mathrm{x}, \mathrm{y}$ and $\mathrm{z}$ axes, the RMSE, 


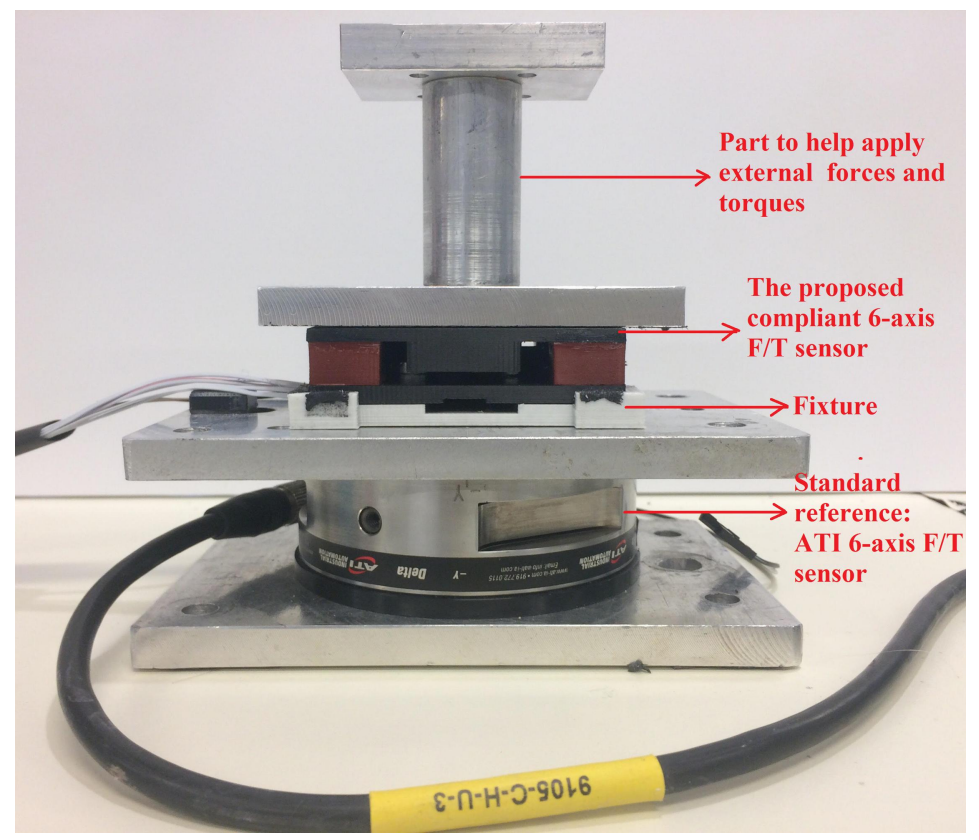

Figure 5.7: Experimental setup for online data collection. The developed sensor is mounted to a standard 6-axis F/T sensor reference from Industrial Automation. Both sensors are connected to the same data acquisition system and data acquired using MATLAB, Real-Time Simulink for comparison.

normalized RMSE and the $R^{2}$ were 0.51 N.m (2.82\%) with $R^{2}$ of $98 \%, 0.51$ N.m $(2.96 \%)$ with $R^{2}$ of $96 \%$ and 1.02 N.m $(9.58 \%)$ with $R^{2}$ of $90 \%$ for each linear least squares estimator $\hat{T}_{x}, \hat{T}_{y}$ and $\hat{T}_{z}$ respectively.

Overall, these results indicate a high accuracy with excellent goodness of fit for each axis even though the sensor exhibits a slightly nonlinear behavior due to the deflection-force relationship induced by the elastic material used between the sensor layers to provide the desired flexibility. This can be seen in Figure $5.8-5.13$.

\subsubsection{Nonlinear Regression Using Decision Trees}

Decision Tree $(D T)$ regression is a powerful type of data fitting method that can be used to solve nonlinear regression problems. The success of DT-based regression 

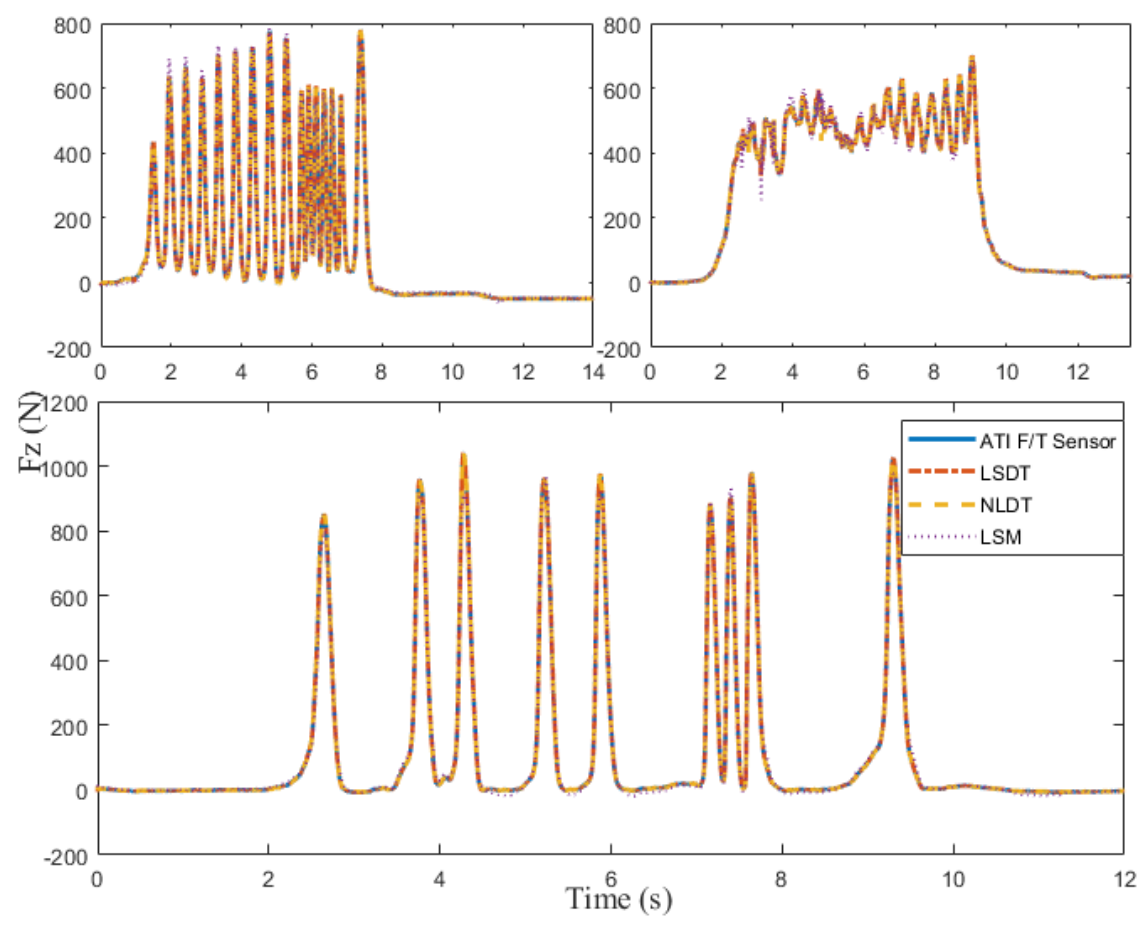

Figure 5.8: Sensor response due to the applied normal-force in the z-direction. The multiple plots show a comparison between three different models and the reference data obtained from ATI 6-axis $\mathrm{F} / \mathrm{T}$ sensor. The models include the novel Least Secures Decision Trees $(L S D T)$, Least Squares Method $(L S M)$ only and Nonlinear Decision Trees NLDT alone.

methods can be associated to their straightforward application, efficient operation, ease of understanding and its hierarchical, branching structure resembling the human decision-making process [225, 226].

Although a satisfactory force and torque estimator was achieved from linear least squares method, the proposed sensor still exhibits slight nonlinear behaviour of $9.62 \mathrm{~N}$ or $(0.92 \%)$ that the previous simple linear least squares method failed to address. This particularly occurs during rapid transitions of force and torque signals. Therefore, a nonlinear estimation model is necessary to capture these nonlinearities.

In this section, a nonlinear regression using decision trees model is proposed and shows a reduction of up to $62 \%$ in RMSE compared to the conventional 


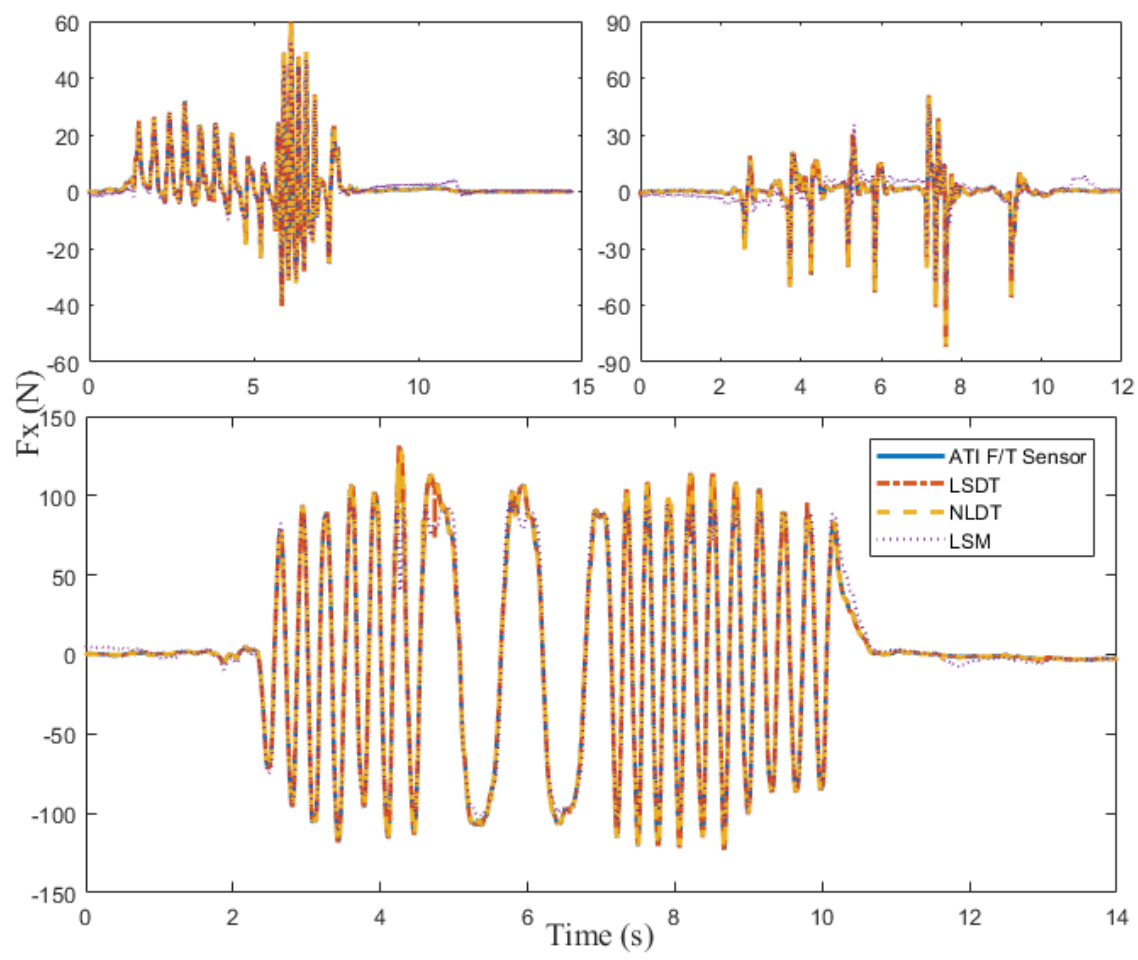

Figure 5.9: A comparison between three models: LSDT, LSM and NLDT of the x-axis with the reference data. Forces different magnitudes, direction and frequencies were applied along the $\mathrm{x}$-axis

linear LSM. A decision tree model was applied in this work as the RMSE and the normalized RMSE of force estimators were reduced from $17.21 \mathrm{~N}(1.65 \%)$ to $7.59 \mathrm{~N}(0.73 \%)$, from $10.02 \mathrm{~N}(7.72 \%)$ to $3.43 \mathrm{~N}(2.65 \%)$ and from $5.44 \mathrm{~N}(5.01 \%)$ to $1.37 \mathrm{~N}(1.64 \%)$ for normal $\hat{F}_{z}$ and shear forces $\hat{F}_{x}$ and $\hat{F}_{y}$ respectively. In terms of torque estimator, the RMSE and the normalized RMSE were also decreased from 0.51 N.m (2.82\%) to 0.1 N.m (0.55\%), from 1.09 N.m (2.96\%) to 0.17 N.m $(0.98 \%)$ and from 1.02 N.m (9.58\%) to 0.11 N.m (1.03\%) in the torques applied about the x, y and z-axis, respectively. 

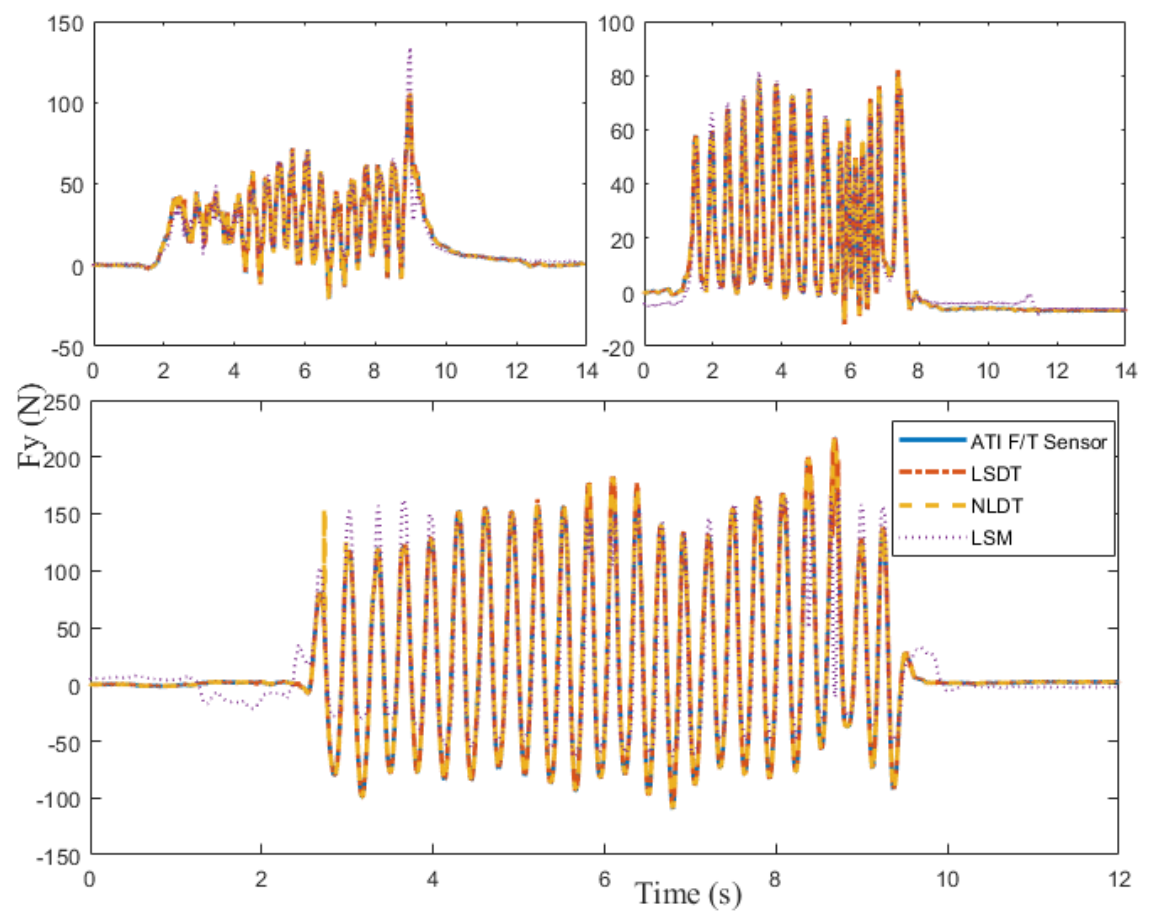

Figure 5.10: Experimental results of the developed prototype for forces applied along the $\mathrm{y}$-axis in a comparison between the estimated and actual data.

\subsubsection{Least Squares Decision Trees LSDT Approach}

After obtaining better force/torque estimation through a nonlinear regression using decision tree model, we propose, a novel and robust calibration methodology to further enhance the precision of the developed sensor performance via a considerable reduction in error. We propose a two-step calibration: a linear least squares estimation model and a concurrent nonlinear estimation model based on DT method, named Least Squares Decision Tree (LSDT).

The largest portion of sensor data is firstly fitted using linear least squares regression. This produces a good and fast linear estimation for forces and torques, but unable to characterize the nonlinear behaviour of the elastic material during large applied dynamic loads. These uncaptured nonlinearities can be defined as the 

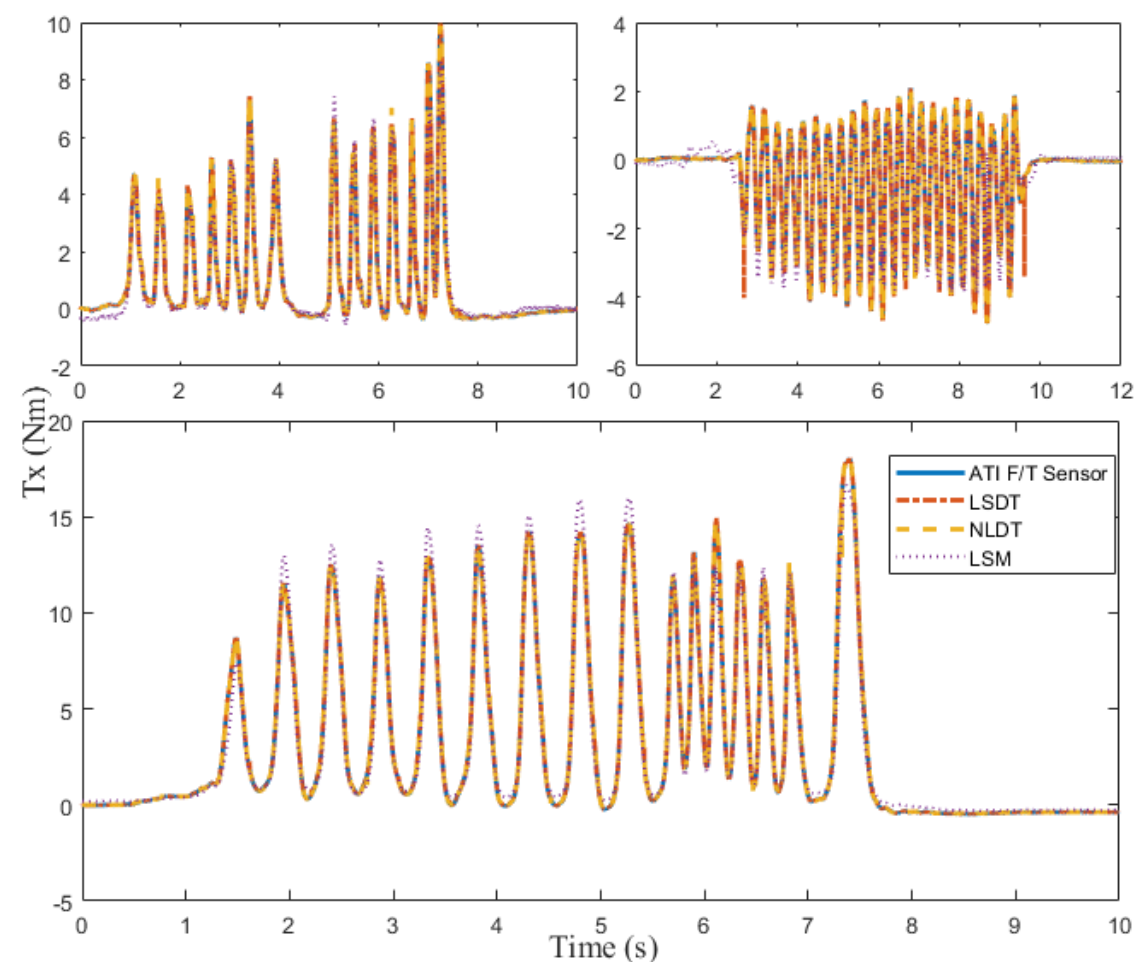

Figure 5.11: Time-domain response of the proposed compliant 6-axis $\mathrm{F} / \mathrm{T}$ sensor due to applied torques around the $\mathrm{x}$-axis. A comparison between the estimated data using the proposed estimation models and the measured data from the reference.

differences between the actual applied forces/torques and the estimated data of the least squares regression (residuals). A decision tree model is used to generate a nonlinear estimator for the residuals. The Least Squares Decision Trees calibration procedure can be described in two steps, as follows:

1. Developing the errors estimator by using the output voltage signal of the proposed sensor as an input to a decision tree model and residuals of the least squares model as the output target. The decision trees model is then trained with a large samples set. It is a one-time training process as the estimated residuals are predicted only from the output voltage signals of the developed sensor.

2. Combining the outputs of linear least squares estimator with the output of the 

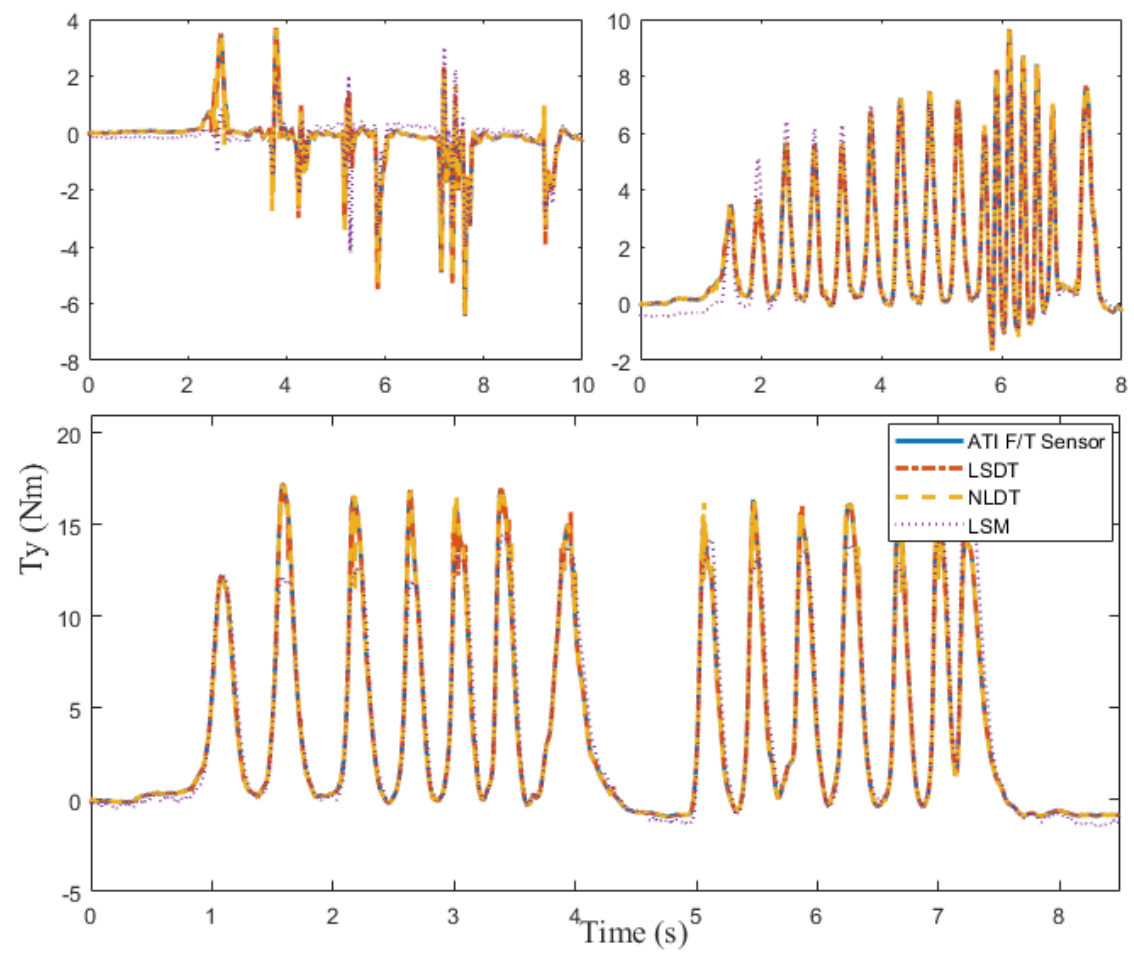

Figure 5.12: Response of the prototype under torques with different magnitudes and directions applied around the y-axis.

nonlinear decision trees model (residuals estimator) so that the output of the proposed calibration methodology becomes the sum of the two outputs as shown in Figure 5.14.

The final estimated force and torque using the $L S D T$ approach can be described in the following equation:

$$
\hat{F / T_{L S D T}}=\hat{F / T_{L S M}}+\hat{\xi}
$$

Where $F / T_{L S D T}$ is the forces and torques estimator of the proposed new calibration methodology, $L S D T ; F \hat{T}_{L S M}$ is the output of the least squares method and $\hat{\xi}$ is the estimated difference between the actual force/torque and linear fitting (residuals estimator). 


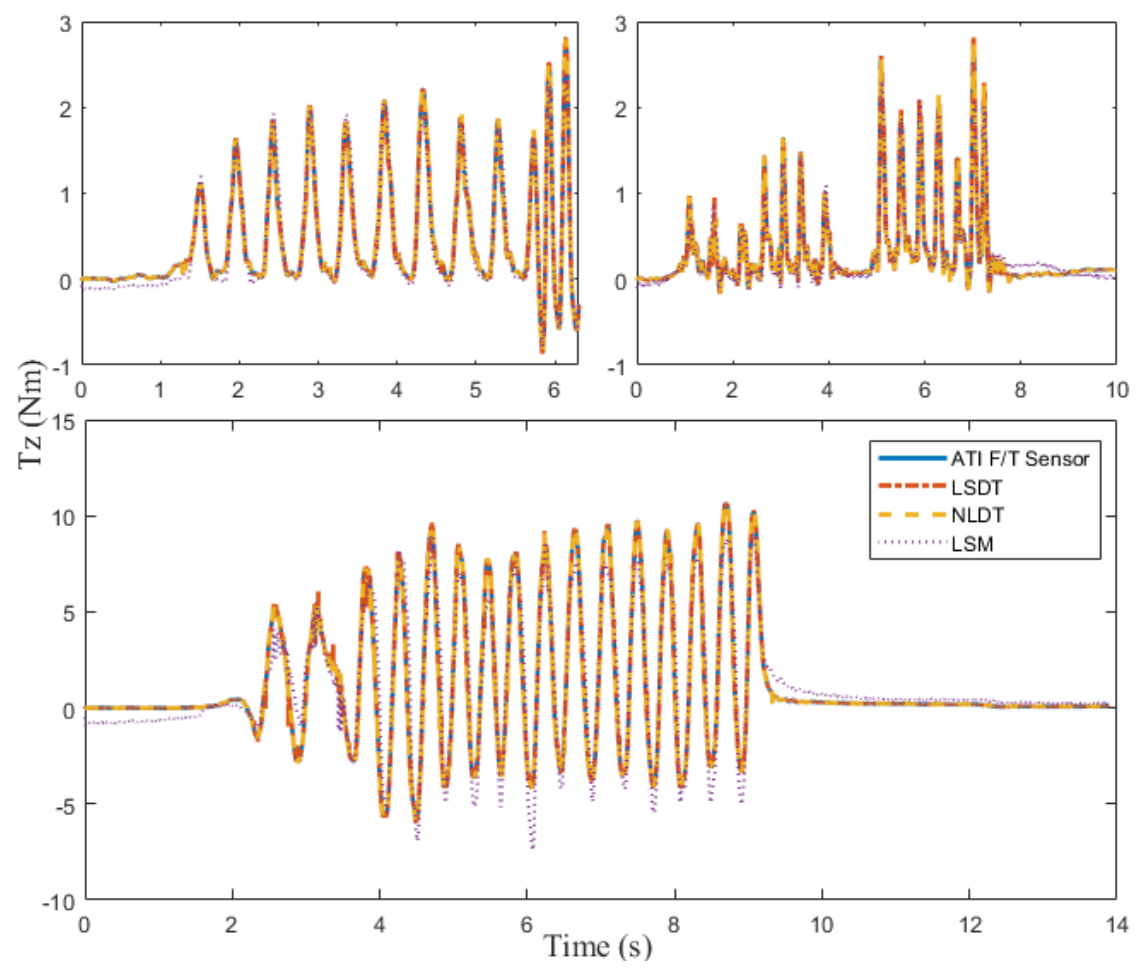

Figure 5.13: Experimental results of the developed sensor for a comparison between actual data obtained from the reference and predicted data achieved from the sensor after applying three estimation models. The graphs demonstrate sensor performance under several torques around the z-axis.

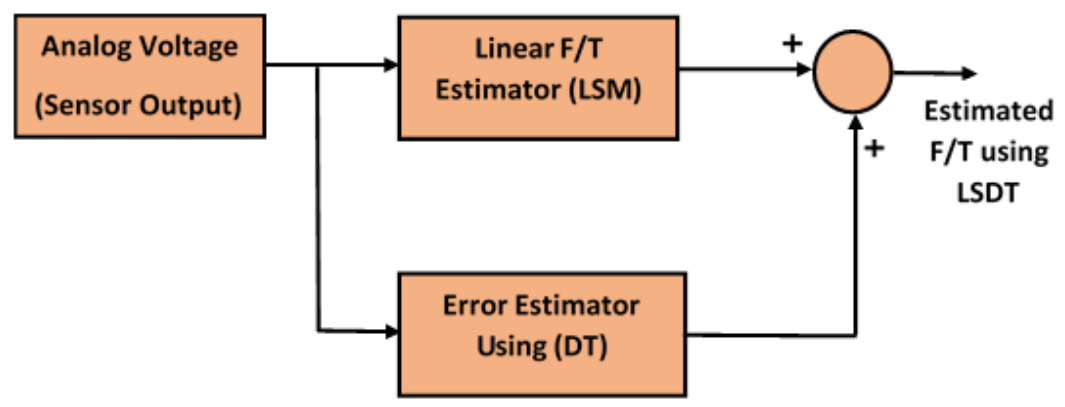

Figure 5.14: Sensor characterization procedure of the novel calibration methodology named as Least Squares Decision Trees $(L S D T)$. Force and Torque data (voltages) acquired from the developed sensor are used as multi-inputs to two models: linear Least Squares model to estimate the applied forces and torques and nonlinear estimation model based on Decision Trees regression to estimated the errors. The sum of the two models is the novel $L S D T$ calibration approach. 

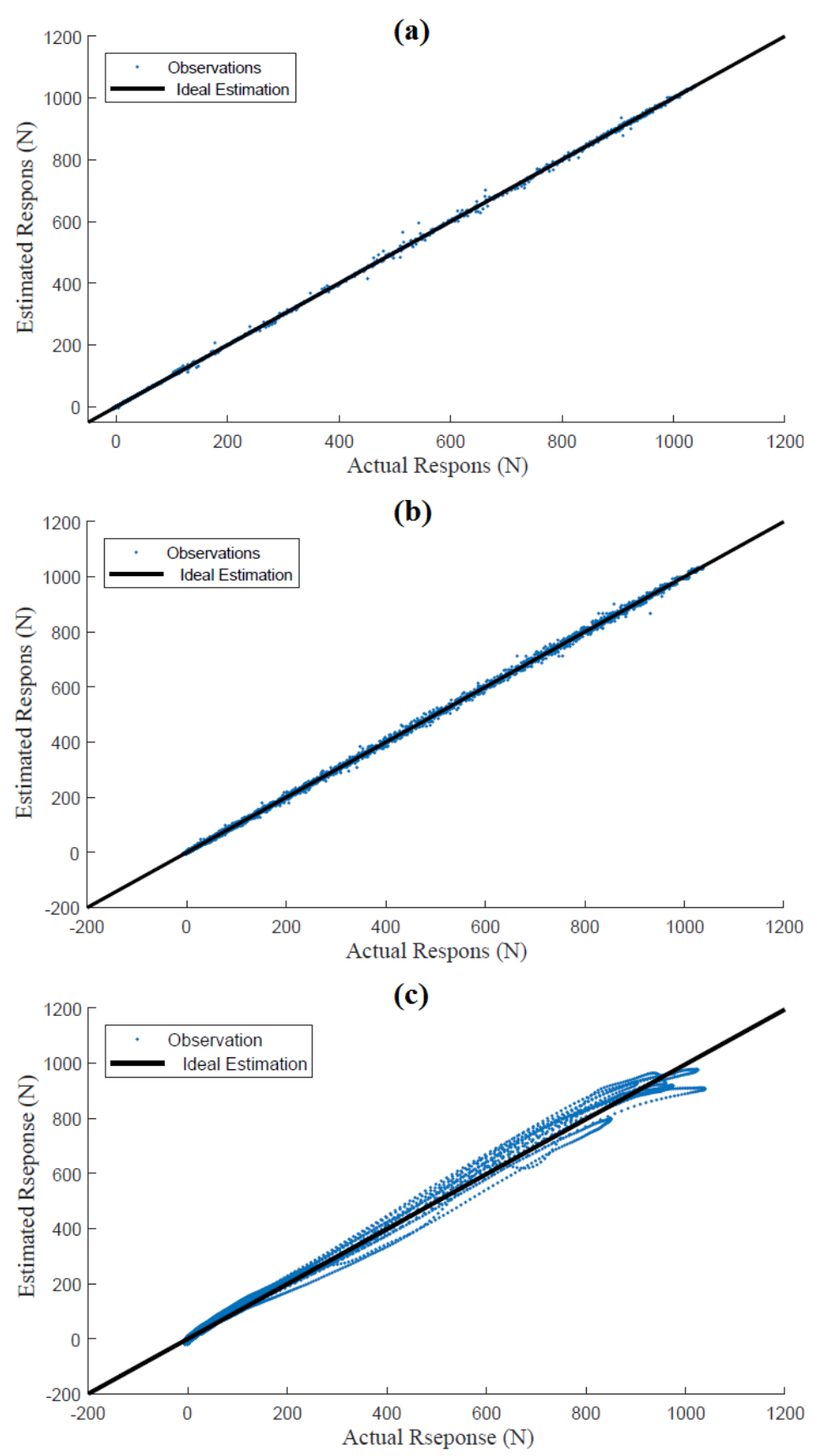

Figure 5.15: The estimated sensor response vs the actual response of the reference for $F z$ estimators. (a) demonstrated the performance of the proposed Least Squares Decision Trees $(L S D T)$. Curves are not easy to distinguish because the model perfectly estimates data. (b) shows the output of the DT model only, which also shows an excellent predilection. (c) Presents the conventional linear $L S M$ model which exhibits slightly less accurate results. 
The Least Squares Decision Tree estimator ( $L S D T)$ gave the best performance with high accuracy and considerably small errors. In comparison with the performance of the Decision Tree $(D T)$ only, the $R M S E$ and the normalized $R M S E$ were extremely reduced from $7.59 \mathrm{~N}(0.73 \%)$ to $1.82 \mathrm{~N}(0.17 \%)$ for the $\mathrm{z}$-axis estimator and from $3.43 \mathrm{~N}(2.65 \%)$ to $0.94 \mathrm{~N}(0.72 \%)$ and from $1.73 \mathrm{~N}(1.64 \%)$ to $0.48 \mathrm{~N}(0.56 \%)$ in the $\mathrm{x}$ - and $\mathrm{y}$-axis, respectively. The estimated torques were also significantly improved as the RMSE and the normalized RMSE were diminished from 0.1 N.m $(0.55 \%)$ to 0.03 N.m $(0.16 \%)$, from 0.17 N.m $(0.98 \%)$ to 0.13 N.m $(0.75 \%)$ and from 0.11 N.m (1.03\%) to 0.09 N.m $(0.84 \%)$ in the torques applied around the $\mathrm{x}$, $\mathrm{y}$ and z-axis, respectively.

Significant enhancements in sensor performance is observed when the LSDT method was applied. The best force and torque estimation can be seen in Figure 5.15 which illustrates a comparison between the three methodologies: $L S D T$ in Figure 5.15 (a), $T D$ regression only shown in Figure 5.15 (b) and Figure 5.15 (c) the $L S M$ for the force applied along the z-axis.

The objective of this comparison is to show how close the proposed estimators match the ideal estimation. As it can be seen in the first plot (Figure (a)), the LSTD estimator closely matches the ideal estimation, while in the second plot (Figure (b)), it shows the performance of the $D T$ only which is slightly less identical to the optimum estimation. The nonlinear effects are seen in the third plot (Figure (c)) of the LSM which gives a satisfactory performance with good linear fitting. Table 5.1 and 5.2 summarize the comparisons between the three calibration approaches in terms of the $R M S E$, normalized RMSE and the goodness of fit, $R^{2}(\%)$.

$L S D T$ also improves the computational processing of sensor characterization which 
Table 5.1: A summary of Experimental Results: Goodness of Fit, RMSE and Normalized RMSE for Each Estimation Model of Applied Forces

\begin{tabular}{cccc}
\hline Fz & Goodness-of-Fit $\left(R^{2} \%\right)$ & RMSE $(N)$ & Normalized RMSE \% \\
\hline$L S D T$ & 100 & 1.82 & 0.17 \\
$D T$ & 100 & 7.59 & 0.73 \\
$L S M$ & 99 & 17.21 & 1.65 \\
\hline \hline
\end{tabular}

\begin{tabular}{cccc}
\hline Fx & Goodness-of-Fit $\left(R^{2} \%\right)$ & RMSE $(N)$ & Normalized RMSE \% \\
\hline$L S D T$ & 100 & 0.94 & 0.72 \\
$D T$ & 100 & 3.43 & 2.65 \\
$L S M$ & 97 & 10.02 & 7.72 \\
\hline \hline
\end{tabular}

\begin{tabular}{cccc}
\hline Fy & Goodness-of-Fit $\left(R^{2} \%\right)$ & RMSE $(N)$ & Normalized RMSE \% \\
\hline$L S D T$ & 100 & 0.48 & 0.56 \\
$D T$ & 99 & 1.73 & 1.64 \\
$L S M$ & 93 & 5.44 & 5.16 \\
\hline \hline
\end{tabular}

resulted in an increase in speed of prediction that is defined as observations per second (obs/sec). The estimation speed was significantly increased by over $50 \%$ compared to $D T$ model when it is used alone to estimate forces and torques.

\subsection{Conclusion}

In this chapter, a multi-dimensional force and torque sensor based on fiber-optic sensing technology with a 6-axis sensing capability was proposed and prototyped. Only six fiber-optic sensing elements without any conditioners or amplifications were integrated into a lightweight and compliant sensing system. The sensor was able to reduce the bandwidth of online data transmission due to the limited number of sensing elements 
Table 5.2: A summary of Experimental Results: Goodness of Fit, RMSE and Normalized RMSE for Each Estimation Model of Torques

\begin{tabular}{cccc}
\hline Tx & Goodness-of-Fit $\left(R^{2} \%\right)$ & RMSE (N.m) & Normalized RMSE \% \\
\hline$L S D T$ & 100 & 0.03 & 0.16 \\
$D T$ & 100 & 0.10 & 0.55 \\
$L S M$ & 98 & 0.51 & 2.82 \\
\hline \hline
\end{tabular}

\begin{tabular}{cccc}
\hline Ty & Goodness-of-Fit $\left(R^{2} \%\right)$ & RMSE (N.m) & Normalized RMSE \% \\
\hline$L S D T$ & 100 & 0.13 & 0.75 \\
$D T$ & 99 & 0.17 & 0.98 \\
$L S M$ & 96 & 0.51 & 2.96 \\
\hline \hline
\end{tabular}

\begin{tabular}{cccc}
\hline Tz & Goodness-of-Fit $\left(R^{2} \%\right)$ & RMSE (N.m) & Normalized RMSE \% \\
\hline$L S D T$ & 100 & 0.09 & 0.84 \\
$D T$ & 99 & 0.11 & 1.03 \\
$L S M$ & 90 & 1.02 & 9.58 \\
\hline \hline
\end{tabular}

used which did not exceed six. The simple configuration and design have led to simpler sensor fabrication and straightforward assembly process, resulting in a low price 6-axis $\mathrm{F} / \mathrm{T}$ sensor. The sensor is customizable as it is possible to increase or decrease the size of the sensor structure and to adjust its measurement range based on the intended application.

A novel calibration methodology named Least Squares Decision Tree LSDT was also proposed and applied to obtain a high precision measurement of forces and torques. The calibration procedure was split up into a linear regression part and a nonlinear regression part. The linear fit was performed using the standard linear least method $(L S M)$ and the nonlinear regression part used a Decision Trees $(D T)$ estimation model. Results were compared to a linear regression $(L S M)$ and a nonlinear 
Table 5.3: Estimation Speed Comparison between The Novel LSDT Approach and DT Model Alone for Each Axis

\begin{tabular}{cccc}
\hline Fz & Estimation Speed (obs/sec) & Fx & Estimation Speed (obs/sec) \\
\hline LSDT & 320,000 & $L S D T$ & 290,000 \\
$D T$ & 310,000 & $D T$ & 130,000 \\
\hline \hline
\end{tabular}

\begin{tabular}{cccc}
\hline Fy & Estimation Speed $(\mathrm{obs} / \mathrm{sec})$ & Tx & Estimation Speed (obs/sec) \\
\hline LSDT & 330,000 & LSDT & 380,000 \\
DT & 260,000 & $D T$ & 120,000 \\
\hline \hline
\end{tabular}

\begin{tabular}{cccc}
\hline Ty & Estimation Speed (obs/sec) & Tz & Estimation Speed (obs $/ \mathrm{sec})$ \\
\hline LSDT & 290,000 & LSDT & 390,000 \\
$D T$ & 280,000 & $D T$ & 330,000 \\
\hline \hline
\end{tabular}

a Decision Tree $(D T)$ only, as separate models. The normalized RMSE of LSDT were as follows $F z=0.17 \%, F x=0.72 \%, F y=0.56 \%, T x=0.16 \%, T y=0.75 \%, T z=0.84 \%$ compared to the normalized $R M S$ errors produced by the $D T$ alone which were $F z=0.73 \%, F x=2.65 \%, F y=1.64 \%, T x=0.55 \%, T y=0.98 \%, T z=1.03 \%$ and finally compared to the normalized $R M S E$ of the $L S M: F z=1.65 \%, F x=7.72 \%, F y=5.16 \%$, $T x=2.82 \%, T y=2.96 \%, T z=9.58 \%$. A reduction of up to $89.42 \%$ in $R M S$ errors was accomplished when the $L S D T$ was used in sensor characterization. Moreover, the prediction speed was enhanced by $55.17 \%$ compared with the prediction speed of the Decision Trees model alone. All the above-mentioned distinctive features qualify the proposed sensor to be a preferred solution for measuring forces and torques at high precision intended for applications such as biomechanics where lightweight and flexibility are highly desirable. 


\title{
Chapter 6
}

\section{Development and Characterization of}

\author{
Compliant FBG-Based, Shear and
}

Normal Force Sensing Elements for

Biomechanical Applications

\begin{abstract}
Fiber Bragg grating (FBG) based sensors rely on the wavelength variation of the optical signal as their main sensing technology. They also use the optical fiber both as a mean of carrying the sensing information through the optical signals and as a sensing element at the same time which is created along the same optical fiber. One of the most attractive advantages of the FBG sensing systems is their distinctive multiplexing capabilities. This means that a large number of FBG sensing elements can be inscribed
\end{abstract}


along only a single optical fiber [227-230]. Sharing one interrogator between several FBG sensing points, therefore decreasing the cost per sensing element of the sensor system, making such sensing technology a competitive, compelling in many cases.

Optical fiber Bragg gratings are being developed with high quality performance in the sensing fields, with wide range of various applications in different areas of physical sensing such as strain, temperature, force and pressure [231-233].

This chapter presents the development process and the evaluation of compliant Fiber Bragg Gratings (FBG) based, normal and shear force sensing elements for biomechanical measurements with their effective novel calibration methodology and preliminary experimental results. The sensing elements are intended for human machine interaction such as in transfemoral prosthetic interface. The sensing elements are designed to utilize the generated deflections due to the applied force to stretch the optical fiber when a normal or shear force is applied on the sensor's top surface. The performance of the sensor is evaluated through a series of experiments including both dynamic and static loading conditions. The experimental results showed that the fabricated sensing elements have the ability to measure applied normal and shear force. Due to the deformable material used, the sensor exhibits slightly nonlinear behaviour between force and deformation. This has been addressed using a novel calibration procedure composed of a linear model to characterize the main sensor data and a nonlinear estimation model based of a nonlinear autoregressive exogenous (NARX) model to simultaneously estimate the errors from the input wavelength data in real-time. The results achieved from the proposed calibration method have revealed an improvement from an R-squared value of $93 \%$ to $100 \%$ when compared to a data obtained using a linear least squares method. 


\subsection{Introduction}

Interface shear and normal forces are essential aspects in the development of human-machine interface of lower-limb assistive devices. Monitoring force distribution at the limb-machine's contact surface can provide important information and valuable knowledge about the biomechanical interaction. In doing so, we can augment the fundamental understanding, helping researchers and clinicians alike; as well as help to improve our progress in the technology of assistive devices. The contact surface force measurements can also help with stump-socket fits, which is considered one of the most important issues for prosthetists that influences the mobility, the quality of life and well-being of amputees [234, 235].

The lack of the shear force measurement is a significant limitation in prosthetic sockets force sensing systems. Residual limbs are subject to considerable amounts of static and dynamic shear forces in addition to the applied normal force. Normal and shear forces acting on the surface of the residual could potentially damage stump tissues since the total ground reaction force is ultimately transmitted through these soft tissues of the residual-limb. Excessive ground reaction forces have revealed to cause an occlusion in blood flow, resulting in stump ulceration and necrosis [236].

Several sensing systems based on different sensing technologies have been developed and proposed to provide a crucial sensory information about human-machine interface forces. Such sensing systems typically depend on thin film pressure transducers which rely on piezoresistive principles [237, 238]. Other sensors were fabricated based on capacitive sensing techniques [239-241]. However, these sensors are highly affected by electromagnetic interference, exhibiting a limited accuracy, nonlinearity errors. 
Force sensitive resistors (FSRs) have also been used in lower-limb human-machine interface to measure the interaction forces between the residual limb and prosthetic socket since these sensors have simple interface and low manufacturing cost. In [242, 243], a set of FSR transducers were integrated into prosthetic socket to measure the distributed interaction force on a residual limb. However, the prototypes were not capable of detecting the two components of shear forces in the $\mathrm{x}$ - and $\mathrm{y}$-directions as they were only able to measure the normal force. In addition, the material characteristics of FSRs are fragile and highly affected by the external environment [244]. The commercially available F-Socket pressure sensors such as Rincoe socket fitting system from Tekscan Inc, are designed for prosthetic socket to measure the pressure distribution on the stump-socket also have the advantages of these sensors are their small thickness, flexibility, acceptable resolution, sensitivity and high bandwidth [245]. The disadvantages of these systems are usually signal creep, their affecting hysteresis and unknown force cross-talk effects [246]. Furthermore, F-Socket pressure sensors are highly affected by water which has a high chance of occurrence due to sweat.

Strain gauge-based transducers have been reported in [247] and [248], where a group of strain gauge-based sensing elements were distributed along a prosthetic socket to measure the applied interaction forces. These transducers are made of metal with a high rigidity which can disturb the amputee's natural gait pattern, leading to uncomfortable walking. These sensors also have weight concerns and are large in size, lying several centimetres out of the stump-socket which causes discomfort and fatigue to the amputee. Attaching heavy mass to the prosthetic socket is not desirable.

Shear forces are also crucial force measurement parameters that are used in biomedical applications such as surgical robotics and prosthetic sensing [249-255], and we endeavour to use FBG as such. The shear force measurements play a major role 
in preventing foot ulceration which are a high risk for diabetic individuals as they experience loss of sensation, particularly underneath the feet [256-263]. Shear force sensors are used to compensate for that lost sensation which may prevent, in some cases, foot amputation surgeries. Therefore, developing a reliable shear force sensor is essential for such biomechanical applications.

Optical sensing technologies such as Fiber Bragg Grating (FBG) based sensors can be a good alternative to the previously stated conventional sensors for stump-socket interface force measurement. Its small size, lightweight, multiplexing capabilities and precision measurement with high sensitivity to external strain makes it a good alternative. More importantly, its unique biocompatibility and its immunity to electromagnetic interference make this technology a well-suited solution for biomechanical applications. This sensing technology is also electrically passive, offering an excellent electrical isolation; and it is inherently safe to be in direct contact with the human body [6]. One disadvantage of this technology is the cost of the required integration system. FBG-based sensors have already been used to assess human-machine interface force [110, 264]. However, these sensors have the limitation of measuring one force component applied in the normal direction. They do not detect any shear forces, which are considered crucial information for stump-socket interface.

In this chapter, we propose the development of compliant, small size, lightweight FBG-based sensing elements capable of measuring both normal and shear forces and their effective calibration methodology to overcome some of the limitations of the aforementioned force sensing systems. The presented sensing elements have the ability to measure normal and shear force components in real-time with RMSE ranging from $2.73 \%$ to $4.57 \%$. The nonlinearity effects due to the large deflection was investigated using both a linear, and a nonlinear calibration method and compared with the 
proposed novel calibration methodology.

\subsection{FBG-Based Normal and Shear Force Sensing Ele- ments: Design and Fabrication}

Figure 6.1 illustrates the sensor architecture and the design concept of the proposed FBG-based force sensing cell. This sensing unit is designed to measure the normal (vertical) force acting along the z-axis. There are several potential applications where such compliant sensors are desired, for example in human-robot collisions detection, plantar normal force mapping in ground reaction force and gait measurement, human machine interaction (HMI) and other rehabilitation systems. The sensor is composed of three main parts: an optical fiber with FBG inscribed on it, a main sensing body (the sensing cell) and silicon rubber. The optical fiber used in this sensor is SMF-28 from Corning, USA. It is a single mode optical fiber with a core diameter of $8.2 \mu \mathrm{m}$ and cladding diameter of $125 \mu \mathrm{m}$.

The main body was 3D printed using Thermoplastic Poly Urethane (TPU) a thermo plastic elastomer or a flexible material. This type of $3 \mathrm{D}$ printing material has the advantage of being able to control the flexibility of the printed model by adjusting the infill density through the software of the 3D printer.

The sensing body has dimensions of $1.38 \mathrm{~cm} \times 2.4 \mathrm{~cm}$ with a thickness of only 0.5 $\mathrm{cm}$. On each of the rounded sides of the sensing body, there is a hole centered with a diameter of $1 \mathrm{~mm}$ made to allow for the optical fiber to pass through. The fiber is then slightly pre-stretched and set by applying glue on each end of the fiber.

Finally, the main sensing body is filled with a flexible material made of Econ 60, a 

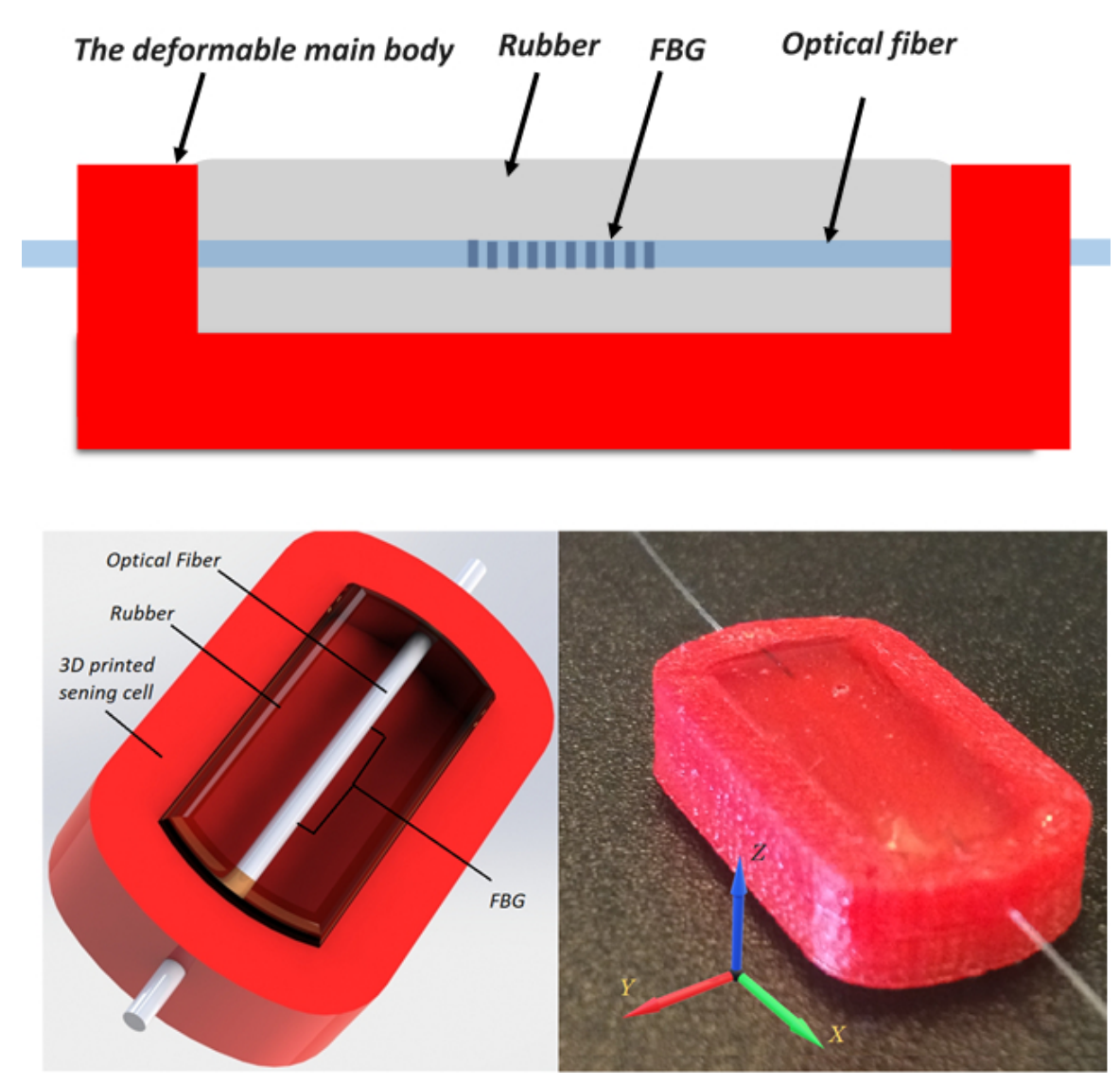

Figure 6.1: Structure of the proposed fiber Bragg grating-based compliant normal force sensor. the sensor is composed of three parts: optical fiber with inscribed FBG sensing region, flexible sensing body and a rubber material used to fill the sensing body. 
liquid urethane rubber with shore hardness 60A from Smooth-On, Inc. The urethane rubber is prepared by thoroughly mixing the Econ 60 with its curing agent in a certain weight ratio, then cured at the room temperature for more that 16 hours. The mixing ratio as provided from the manufacturer, and in this case is 1:1 for the urethane rubber Econ 60 and the curing agent, respectively. The fabricated final product force sensor element is shown in Figure 6.1.

The FBG was inscribed in hydrogen-loaded SMF-28 (the single mode optical fiber) using a krypton Fluoride ( $\mathrm{KrF}$ ) excimer laser (type of ultraviolet light) with a wavelength of $248 \mathrm{~nm}$ using a conventional FBG fabrication method of the phase mask. The length of the FBG is $3 \mathrm{~mm}$ at a wavelength of $1543 \mathrm{~nm}$ (period of phase mask is $1066 \mathrm{~nm}$ ). After the FBG was written along the optical fiber, it was put in an oven at $120{ }^{\circ} \mathrm{C}$ for 12 hours to remove the remaining hydrogen.

The proposed FBG-based force sensing element is designed and fabricated to measure the normal force.

A small size, lightweight, FBG-based shear force sensing cell was also designed, fabricated, and successfully characterized to incorporate shear force sensing. Figure 6.2 demonstrates the design concepts of the proposed FBG-based shear force sensing element which is also composed of the main sensing body of the same dimension as the previous FBG-based, normal force sensing cell.

However, the shear force sensing cell contains a small element built on the inner surface of the main sensing body, used to detect shear force. This element has a centered hole, $0.5 \mathrm{~mm}$ in diameter, for the optical fiber with the FBG sensing region to pass through. The fiber is then pre-stretched and glued in that hole at both ends. The fiber remains moderately stretched for several hours until the glue is completely 

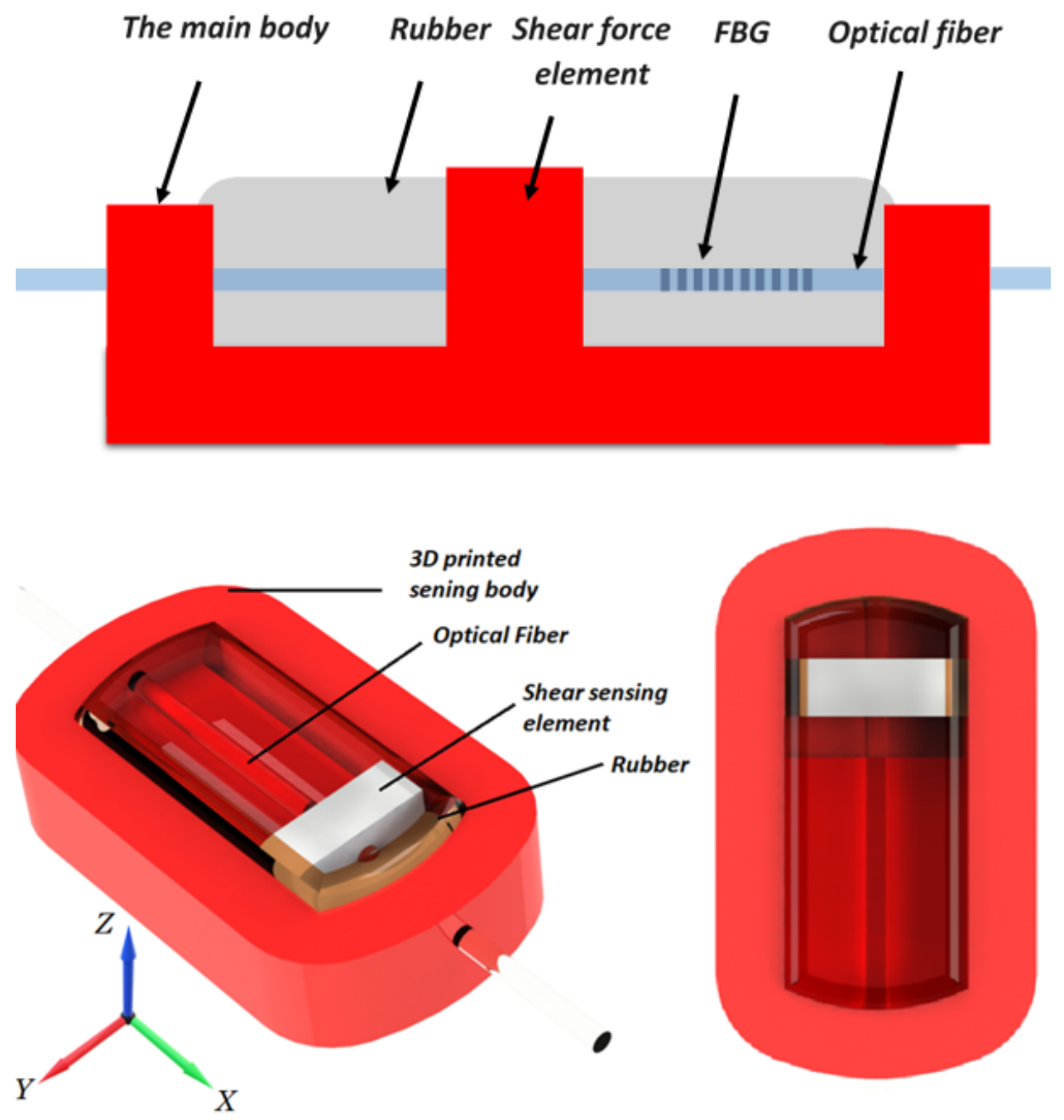

Figure 6.2: Structure of the proposed fiber Bragg grating-based shear force sesning element. the sensor is composed of three parts: optical fiber with inscribed FBG sensing region,sensing body and a rubber used to fill the sensing body. 
dry. Once the optical fiber is affixed in that hole at both ends, the liquid urethane rubber Econ 60 is used to fill the sensing body including the shear force element which can be seen in Figure 6.2.

\subsection{Sensing Mechanism and Operating Principles}

The fiber Bragg gratings (FBG) are narrow-band light filters that are permanently inscribed along the core of a single mode optical fiber. The resulting inscribed core or grating causes the light that passes through to be partially reflected. The maximum reflection occurs when each portion of the reflected light (from each grating) is in-phase with each other. This reflected wavelength is called Bragg wavelength. Any external disruption that can alter the periodicity of the gratings (distance between each grating) or the refractive index will result in a proportional shift in the reflected Bragg wavelength. The Bragg wavelength's shift represents a measure of the applied external perturbation.

When an optical fiber with a FBG is stretched due to an applied axial strain, the distance between each grating changes, the reflected Bragg wavelength changes, and it provides a satisfactory measurement for the applied axial strain.

Figure 6.3 demonstrates the sensing mechanism and working principles of the proposed FBG-based sensor designed to measure the normal force applied along the z-axis, where only one FBG sensing element inscribed along a single mode optical fiber is used to measure the applied vertical force. The FBG sensing region is located inside the main body of the sensor (at the center) and the fiber is affixed at each end of the sensor body. When the top surface of the sensor is excited by a normal force acting 


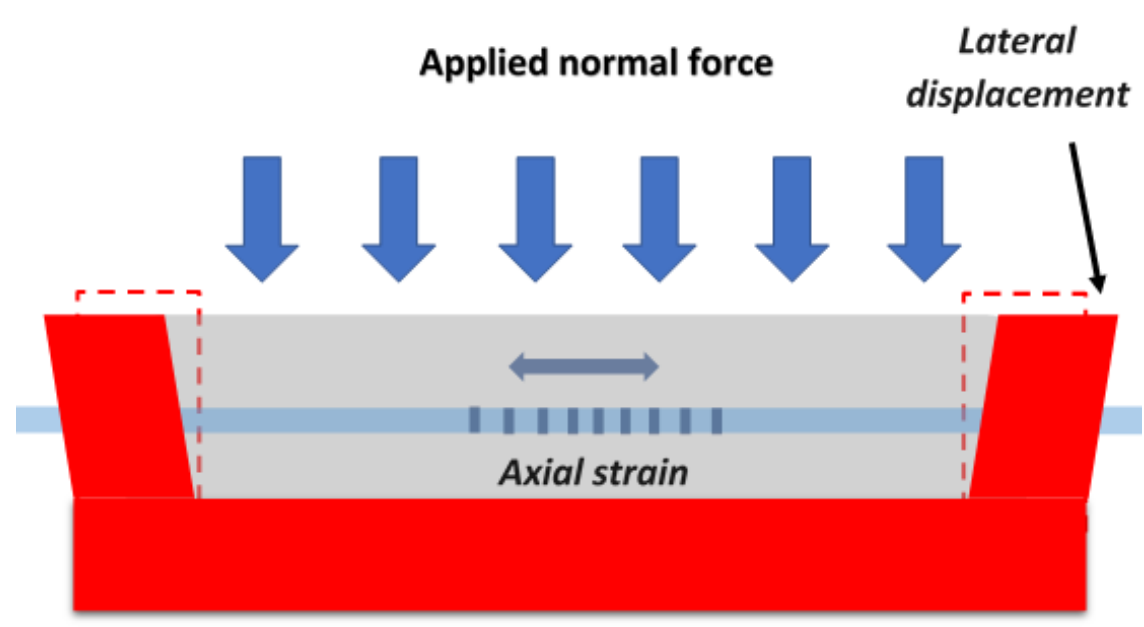

(a)

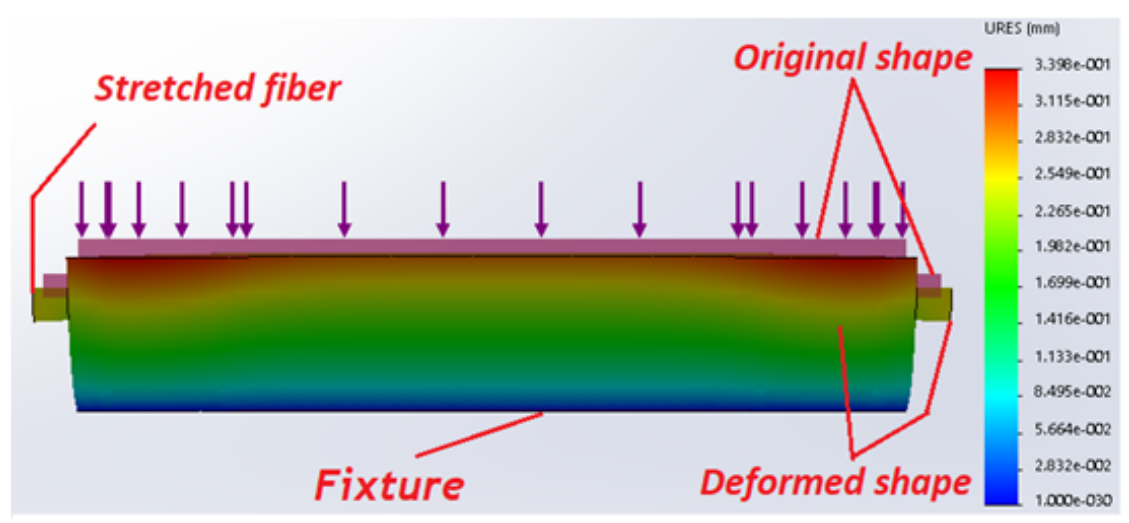

(b)

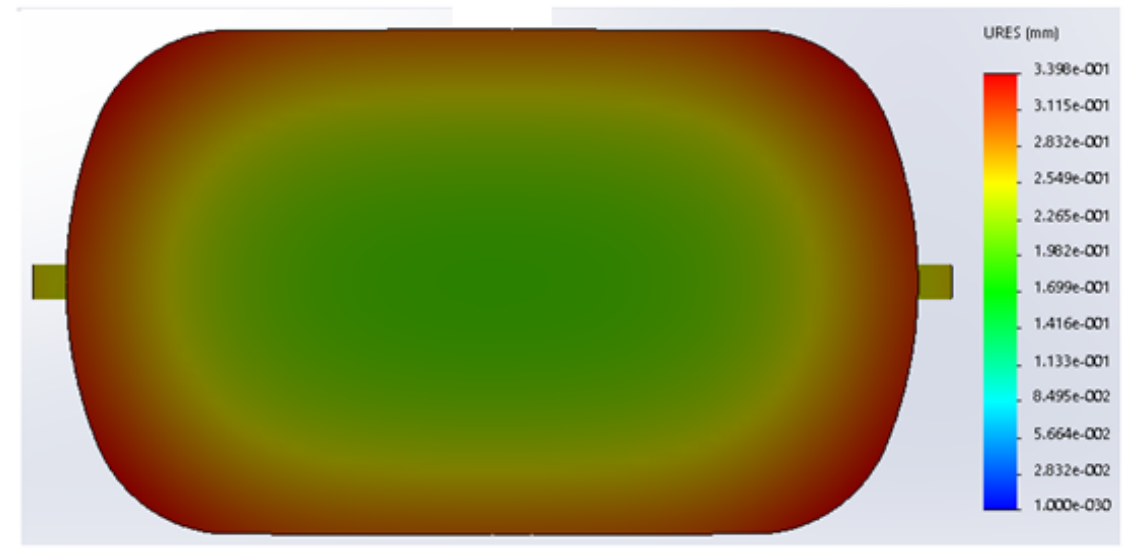

(c)

Figure 6.3: Illustration of the sensing mechanism of the proposed FBG-based force sensor using finite element analysis performed using Solidworks. 
along the z-axis, the main flexible sensing body deflects, generating a displacement at both ends where the fiber is affixed. This will result in stretching the fiber by converting the vertical force to an axial strain acting on the optical fiber embedded into the sensor. As a result, the grating periodicity of the FBG changes, which translates as a measure of the applied force.

Figure 6.3 (a) shows how the original shape of the proposed sensor deforms when a vertical force is applied. This was carried out using Solidworks 's FEA, a CAD software, which is mainly used to explain the working mechanism of both normal and shear force sensing elements. Figure 6.3 (b) illustrates the top view during the applied vertical force. Both illustrations in Figure 6.3 confirm that the largest deflections occur on the areas where the fiber is affixed, and the largest displacement is at the edges where the optical fiber is affixed. Figure 6.4 shows strain results obtained from the FEA.

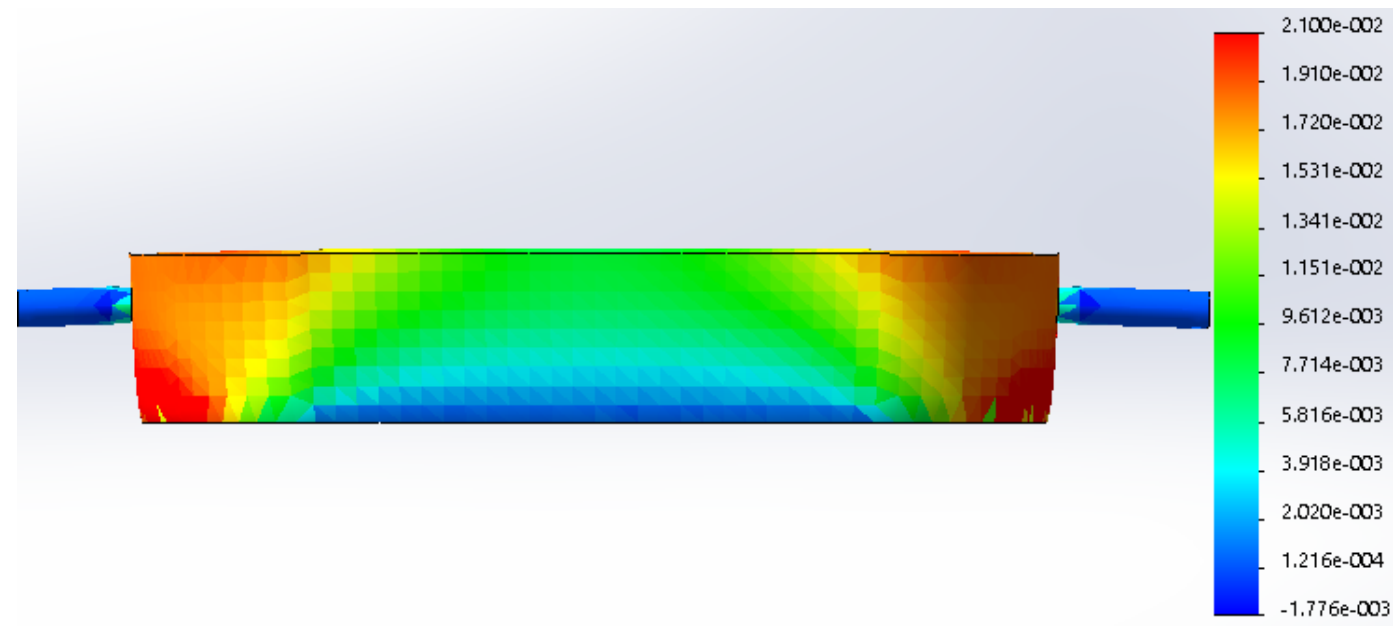

Figure 6.4: Strain distribution along the x-axis due to the applied normal force.

Figure 6.4 illustrates the strain along the $\mathrm{x}$-direction as a result of the applied normal force. In terms of the sensing mechanism of the shear force, the small element that is created in the sensing body of the shear force cell is used to help stretching the optical fiber at the end of the FBG region. When a certain amount of 
force is longitudinally applied along the upper surface of the sensor, a corresponding displacement is generated on the aforementioned small element, which generates an axial strain along the fiber. Since this element works as a cantilever beam which pulls the fiber, producing a wavelength shift proportional to the applied shear force. This can be seen in Figure 6.5.

The necessary types of force sensing elements required to successfully achieve any multi-axis force and torque measurement were developed and prototyped in this work. To obtain a six-axis force and torque sensing system, at least six force sensing elements are needed: three shear force sensors to measure Fx, Fy and Tz; and three normal force sensing elements to measure Fz, Tx, Ty.

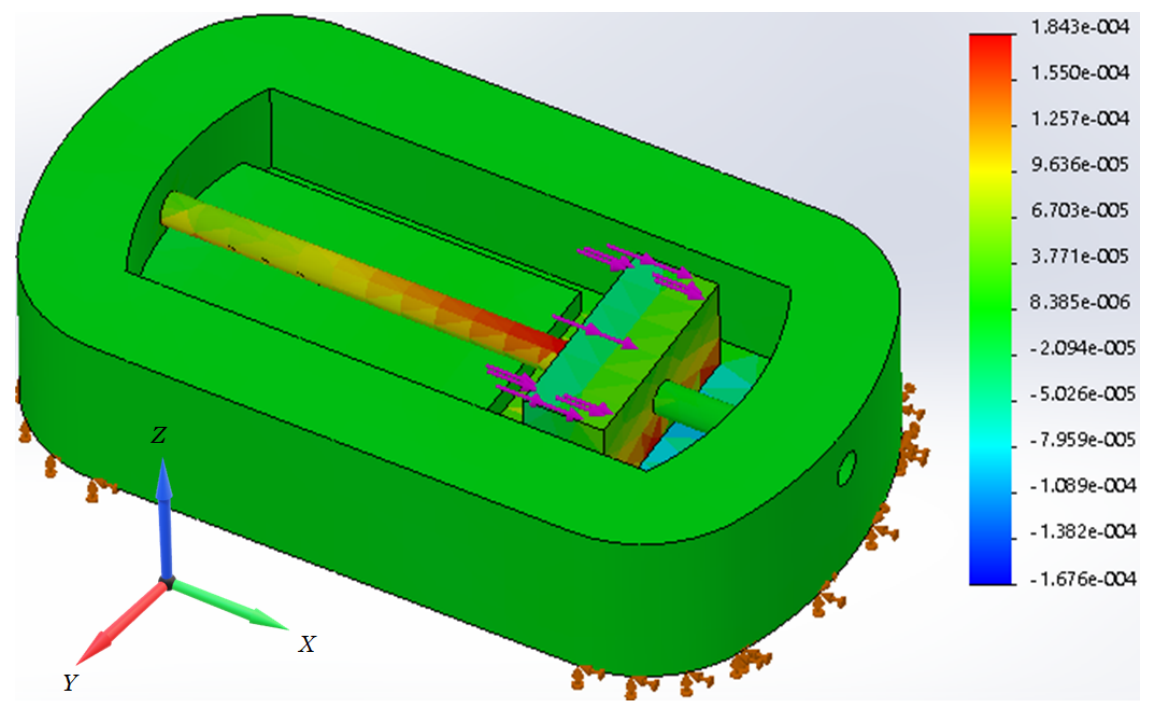

Figure 6.5: Explanation of the sensing mechanism and the strain distribution on the proposed FBG-based shear force sensing element using finite element analysis performed using Solidworks. As it can be clearly seen the optical fiber with the FBG is starched by a small element at the middle of the sensing body when a shear force is applied. 


\subsection{Experimental Setup}

The experimental setup shown in Figure 6.6 is used to characterize the proposed force sensing element and obtain an accurate relationship between the generated reflected wavelength shift and the applied normal and shear force. The applied force is randomized to have forces in the normal and shear directions. A 6-axis force and torque sensor (ATI, Delta SI-60-600, from the Industrial Automation) is used as a

reference. The proposed sensor is positioned on top of the reference sensor. The data acquisition system includes a PCI-6229 16-bit multi-function DAQ card from National Instrument used to acquire data from the ATI 6-Axis force and torque sensor. Raw data is received and further processed using MATLAB Simulink with xPC-Target in real-time environment.

The FBG sensor data was recorded using an optical sensing analyzer (Si720, supplied by Micron Optics), which has high accuracy, high resolution and a high power FBG interrogation system. The equipment has a built-in display system which provides the user with full understanding of the fiber Bragg grating spectrum, and shows how the reflected Bragg wavelength shifts when the physical conditions change. This optical device is used to detect the change in the reflected Bragg wavelength of the proposed sensor. Since the recording speed of the optical sensing analyzer is up to $5 \mathrm{~Hz}$, the sampling rate of the ATI 6-Axis force and torque sensor is set to the same rate to obtain matching data points from each sensor for ease of calibration. Figure 6.9 shows the procedure for shear force application. 


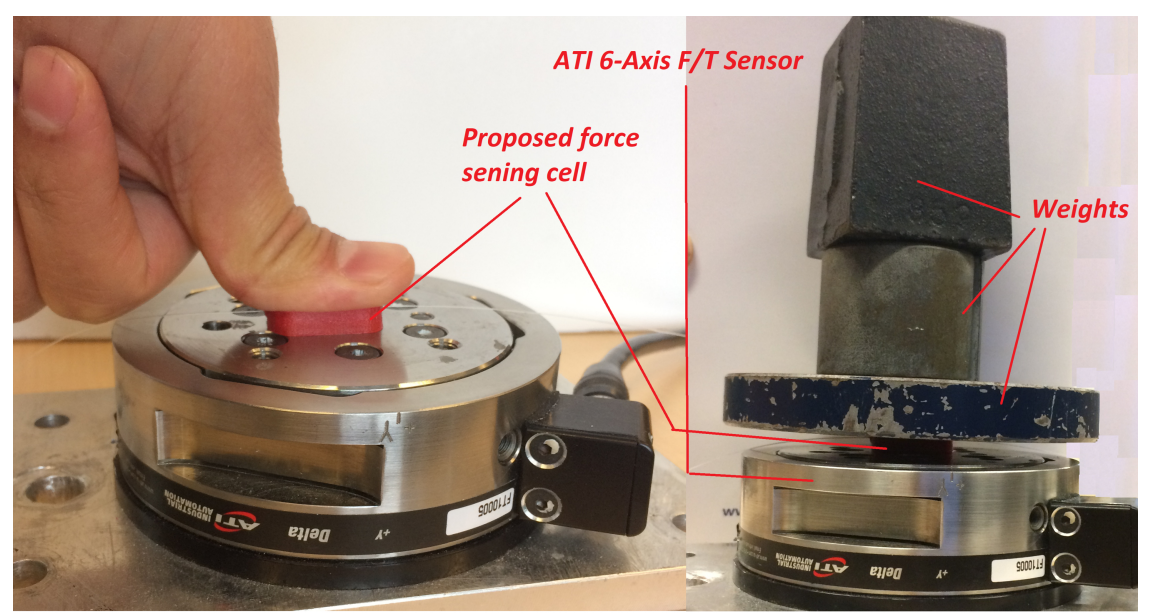

Figure 6.6: The experimental testing steps which are used to test the proposed compliant FBG-based force sensor in both dynamic and static loading conditions. In the dynamic loading, arbitrary vertical forces with different magnitudes are applied on the top surface of the sensor whereas a different weights are used for static test.

\subsection{Sensor Calibration Methodology: Results and Discussion}

As it can be seen in Figure 6.6, two different tests were carried out: static and dynamic tests. In the static calibration, the force was varied from zero up to around $200 \mathrm{~N}$ as shown in Figure 6.7 and Figure 6.8. The weight sets were directly applied to the proposed FBG sensing element by putting them on top of the sensor and gradually increasing the load. While doing so, data was recorded from both sensors, the 6-ATI force and torque sensor and the FBG-based transducer.

Figure 6.7 illustrates the sensor response due to the applied static loads. A linear least squares method was applied to characterize the sensor. The relationship between the applied force and the change in wavelength was linear, as shown in Figure 6.7. The 


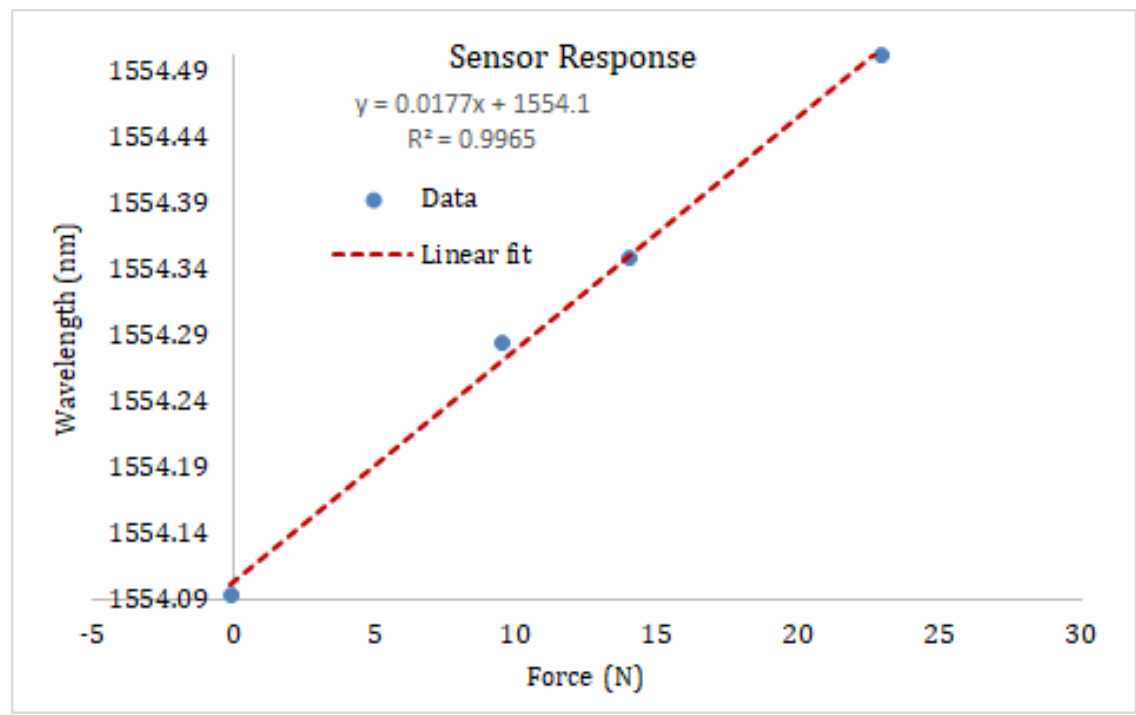

Figure 6.7: The experimental results obtained from static loading conditions test. a linear function used to fit sensor data.

model equation can be characterized as follows:

$$
w=0.017713 * p-1554.1
$$

where $p$ is the measured applied force in Newton and $w$ is the generated wavelength shift in nanometers. The coefficient of determination (goodness of fit, $R^{2}$ ) is $99.5 \%$ with root mean square error (RMSE) of only $0.69 \mathrm{~N}$. The normalized root mean square error (NRMSE\%) is found to be $3.01 \%$.

Overall, the proposed sensor exhibits good fit with a linear relationship. However, this linearity is within the force range of around 0-107 N. This is due to the generated small deflection. The FBG-based sensing elements is embedded into an elastic material (urethane rubber) which exhibits a nonlinear behavior in the case of large deflection which is observed at higher force range. Due to the viscoelastic properties of the rubber used, the behaviour is therefore expected to be nonlinear. Using an applied 
force range of over $180 \mathrm{~N}$, we can animate the proposed sensor's viscoelastic properties or nonllinearity. A quadratic model was able to accurately capture the nonlinearity (in the static conditions) as shown in Figure 6.8. The proposed quadratic model is described by:

$$
w=-4.489 * 10^{-} 5 * p^{2}+0.030 * p+1553.5
$$

This quadratic model is able to precisely describe the nonlinear relationship between the applied normal force and the change in the wavelength. The coefficient of determination is found to be $99.6 \%$ with an RMSE of only $0.10 \mathrm{~N}$.

The proposed sensor is designed to be used inside a lower-limb prosthetic socket or inside a shoe, where the sensor is directly attached to the human body. As a result, the temperature of the sensor ought to be similar to the temperature of the surface of the human body to avoid temperature variation; in such a case, an additional FBG sensor can be added to work as a temperature compensator. Also, the sensing element it self (FBG) is not prone to electromagnetic interference making such sensor suitable to be used for feedback system. Both normal and shear force sensing elements can be integrated into an insole shoe. Such sensing elements are small size and light weight and have the ability to accurately measure shear and normal force. These sensors can be distributed along insole shoe to measure the total ground reaction force.

\subsubsection{Nonlinear System Identifcation using Nonlinear Autoreg- ressive Exogenous NARX Model}

In our previous light intensity modulation-based force and torque sensors, including the 3 - and the 6-axis force and torque sensor [1-3], linear system identification process 


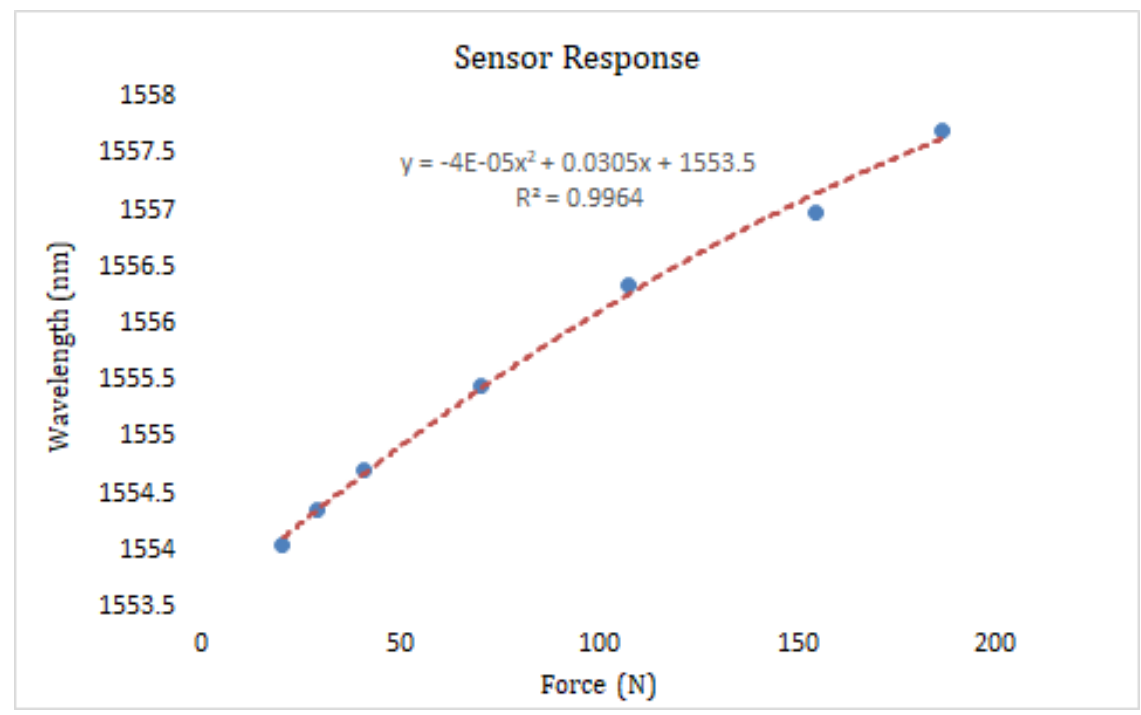

Figure 6.8: The experimental results. The force applied in this case is much higher than the previous which generated in a higher deflection that results in slight nonlinearity. A quadratic function is used to fit the data.

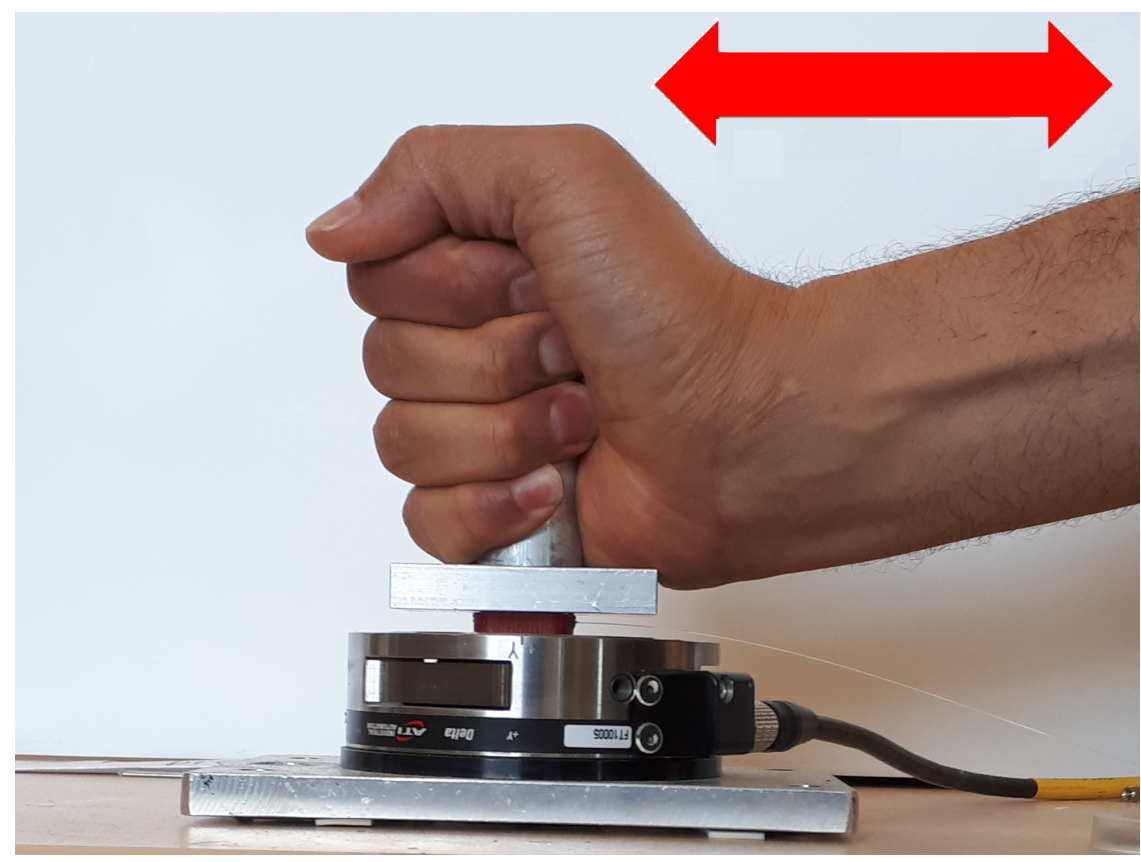

Figure 6.9: The experimental setup used to test the proposed compliant FBG-based shear force sening element force. 
with several linear dynamic estimation modes were applied, obtaining an excellent performance and accurate results. In both sensors prototypes a good linearity was observed even at high force and torque measuring ranges. This was due to the nonlinearity cancelation phenomenon that occurred when the mechanical nonlinearity caused by the elastic martial cancelled the nonlinear correlation between the light intensity and the generated displacement. This phenomenon was not observed in the proposed FBG-based force sensor, therefore linear system identification process is not the best choice for this class of sensor due to the high compliance which results in nonlinear behaviour when a high amount of force is applied.

In most complex nonlinear systems, obtaining a mathematical model that can precisely describe the whole spectrum of the system complexity is not always feasible, not even with a parametric model. Thus, a nonlinear dynamic model using a nonlinear system identification is proposed. A nonlinear autoregressive estimation model with exogenous input (ie. feedback loop), is used to characterize the dynamic nonlinear behavior of the proposed FBG-based force sensor. The nonlinear autoregressive with exogenous inputs model (NARX) is a prediction model based on an ARX estimation model with a nonlinear mapping function which can be selected as a sigmoid, wavelet or neural network function. The structure of the NARX estimation model is shown in Figure 6.10. NARX models have been used in online system identification to identify complex nonlinear systems [265-269], which have confirmed the effectiveness in data driven modelling and system identification of both nonlinear and linear systems [270-272]. The NARX was first introduced as a modern representation for a broad class of nonlinear, discrete systems [273], which was also used in sensor modeling and calibration [274-278] . 


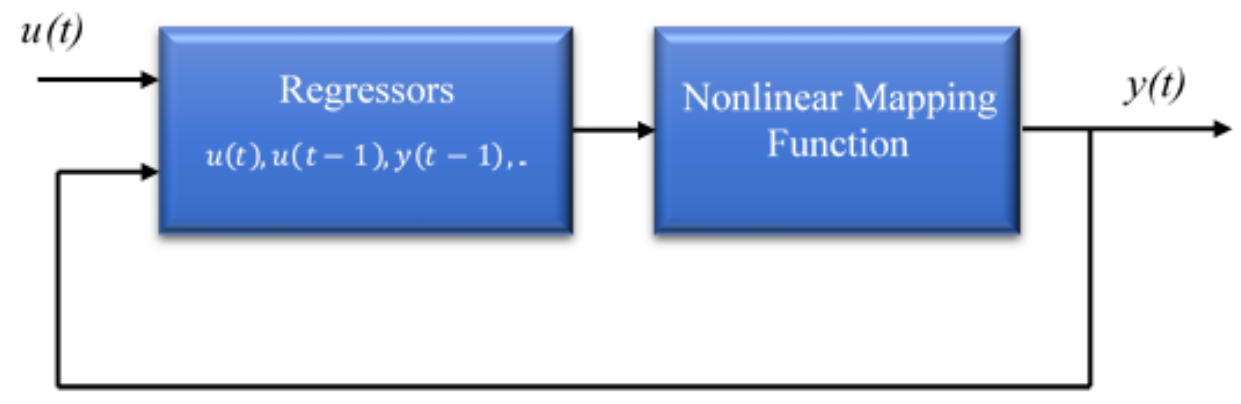

Figure 6.10: The structure of the proposed NARX prediction model model

The structure of the NARX estimation mode contains a number of regressors and a nonlinear mapping function that can be described as follows [279, 280]:

$$
y(t)=G\left(y(t-1), y(t-2) \ldots, y\left(t-n_{y}\right), u(t-1), u(t-2) \ldots, u\left(t-n_{u}\right)\right)
$$

where $y(t)$ and $u(t)$ are the sampled output and input variables respectively, $G$ is the overall function, $n_{y}$ is the maximum number of the past output terms and $n_{u}$ is the maximum number of the past input terms.

Figure 6.11 demonstrates the performance of the FBG-based force sensor when the NARX estimation model is used to characterize the sensor. The results are compared with the force and torque reference sensor (ATI, 6-axis F/T sensor). The proposed sensor was affixed on the top surface of the ATI 6-axis F/T sensor, and data was recorded in real-time from both sensors while a dynamic force with different magnitudes was arbitrarily applied by hand (as opposed to a machine-applied force), as shown in Figure 6.6. The NARX model captures the nonlinearity by describing the complex dynamic behaviour of the sensor and providing a precise force measurement. The model generates small RMSE of only $8.5 \mathrm{~N}$ out of vertical force range of over $300 \mathrm{~N}$. This means that Normalized RMSE (RMSE \%) is $2.73 \%$. The coefficient of determination (goodness of fit, $R^{2 \%}$ ) was found to be $99.30 \%$. 
Figure 6.12 and Figure 6.13 illustrate the sensor's response to altering force signal profiles and different magnitudes. The direct overlap confirms the sensor's ability to describe any externally applied force. This indicates a high quality performance of force measurement can be obtained from the proposed sensor.

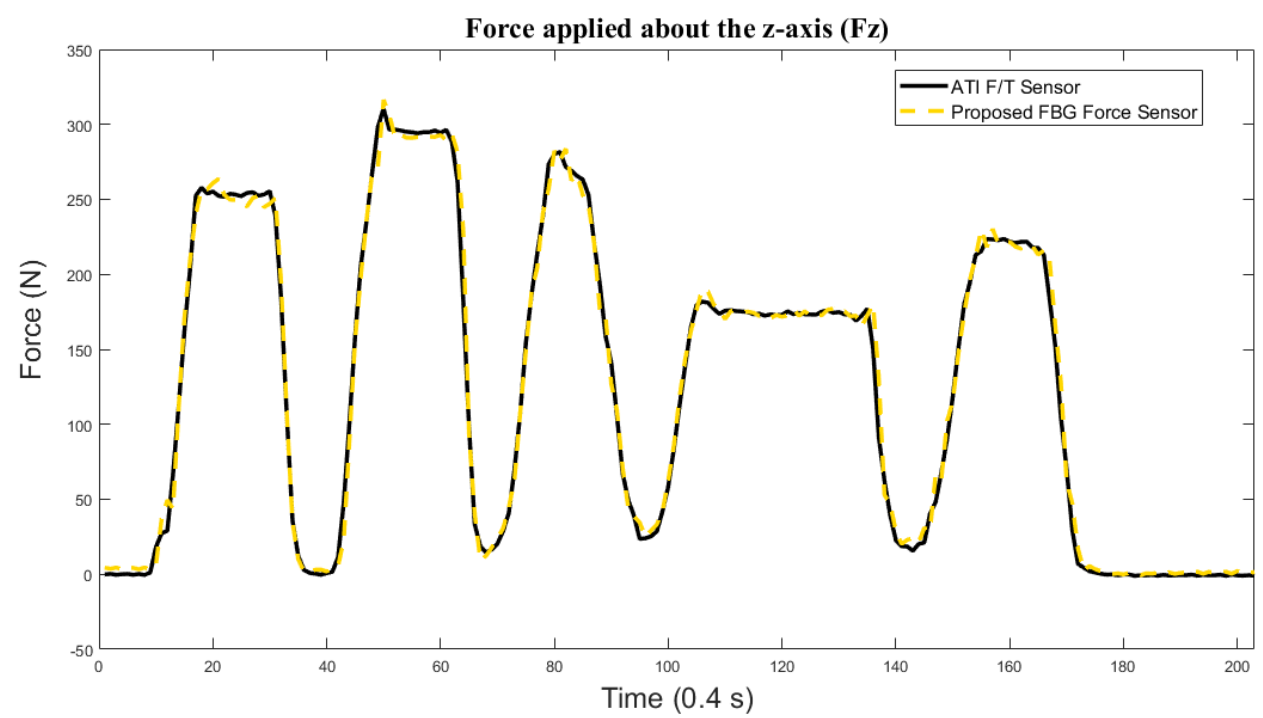

Figure 6.11: The experimental results: A comparison between force measurements obtained from the ATI 6-Axis force and torque sensor and the results of the fabricated FBG-based normal force sensing element using the NARX model

\subsubsection{Linear Regression with A Nonlinear Autoregressive Exogenous (LR-NARX) Calibration Procedure}

To further enhance sensor performance and increase the speed of estimation, a novel calibration methodology is proposed. The calibration procedure is split into two estimation models: a simple linear regression achieved using least squares regression, and a nonlinear regression performed using nonlinear autoregressive with exogenous input model. Figure 6.14 illustrates the structure of the proposed calibration procedure, 


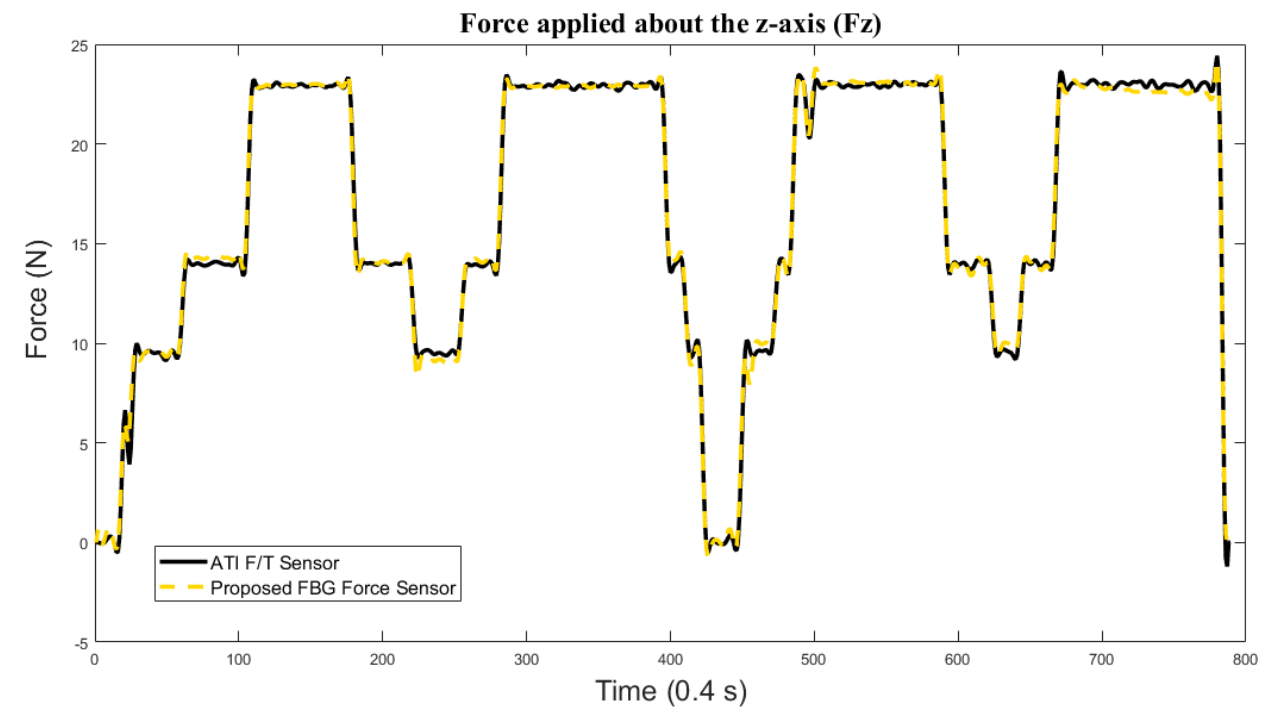

Figure 6.12: The performance of the fabricated FBG-based normal force sensing elements when different amounts of loads (weights) are applied compared to the ATI 6-Axis force and torque sensor.

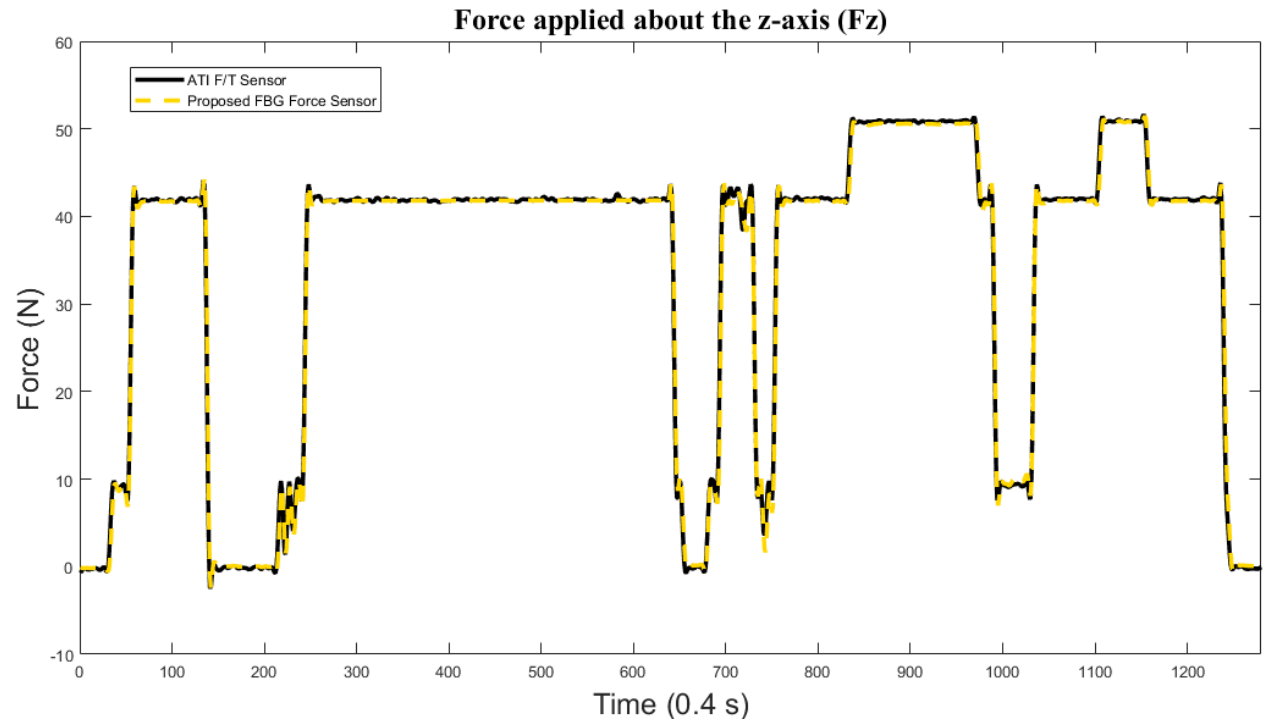

Figure 6.13: A copmarison between the experimental results of the proposed FBG-based sensor and the force reference in real time. 


\section{LR-NARX.}

The static quadratic function is only able to precisely describe the nonlinear behavior of the sensor in the static loading conditions. However, in the case where a dynamic lading is applied, the static function performance is not satisfactory. Therefore, the LR-NARX was used to further improve the sensing performance since it is an integrated static and dynamic model.

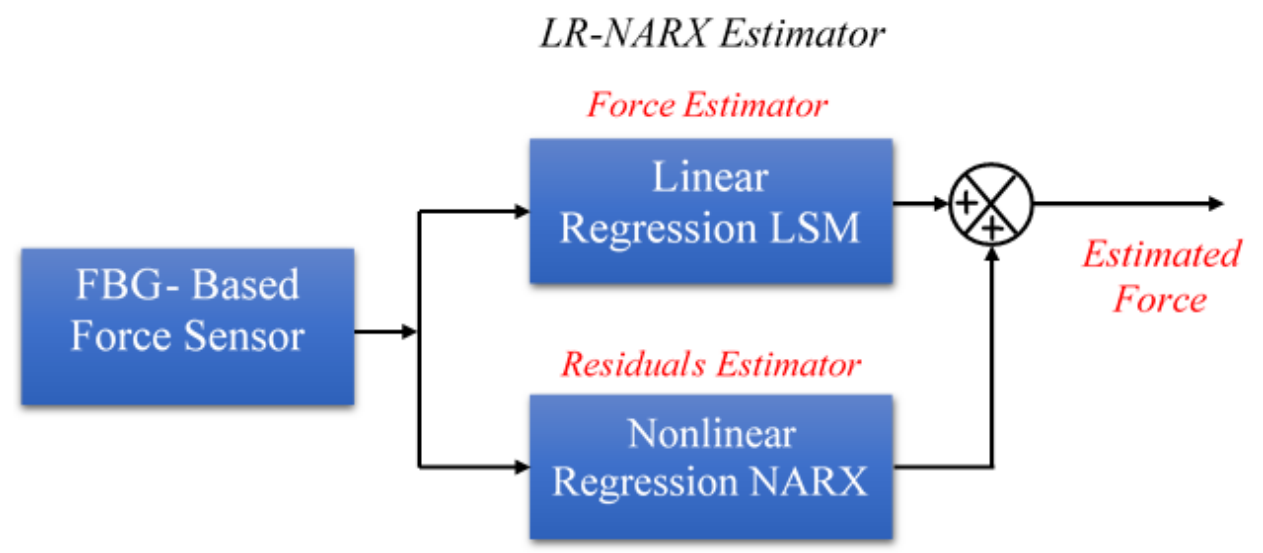

Figure 6.14: The structure of the proposed linear regression with a nonlinear autoregressive exogenous (LR-NARX) calibration procedure.

The linear estimator is used to estimate the force obtained from the linear portion of the sensor data which lies within a force measuring range of 107 N. Having achieved a satisfactory linear force estimation using least squares method, the proposed calibration procedure seeks to further enhance the force estimation by including the nonlinear portion of sensor data which represents the difference between the real force data and the estimated force data obtained from the linear model, in other words the errors. A nonlinear autoregressive with exogenous input model (NARX) is used to predict these errors. The LR-NARX is then the sum of the linear estimator and the nonlinear estimator, depicted in Figure 6.14.

Figure 6.16 demonstrates the sensor performance compared to the ATI 6-axis force 
and torque sensor when the proposed linear regression with a nonlinear autoregressive exogenous (LR-NARX) calibration procedure is used. The coefficient of determination was found to be $100 \%$, which is an improvement compared to the NARX model alone with $99.30 \%$ and the linear least squares regression alone with $93 \%$. The proposed solution shows uncertainty of only $2.23 \%$.

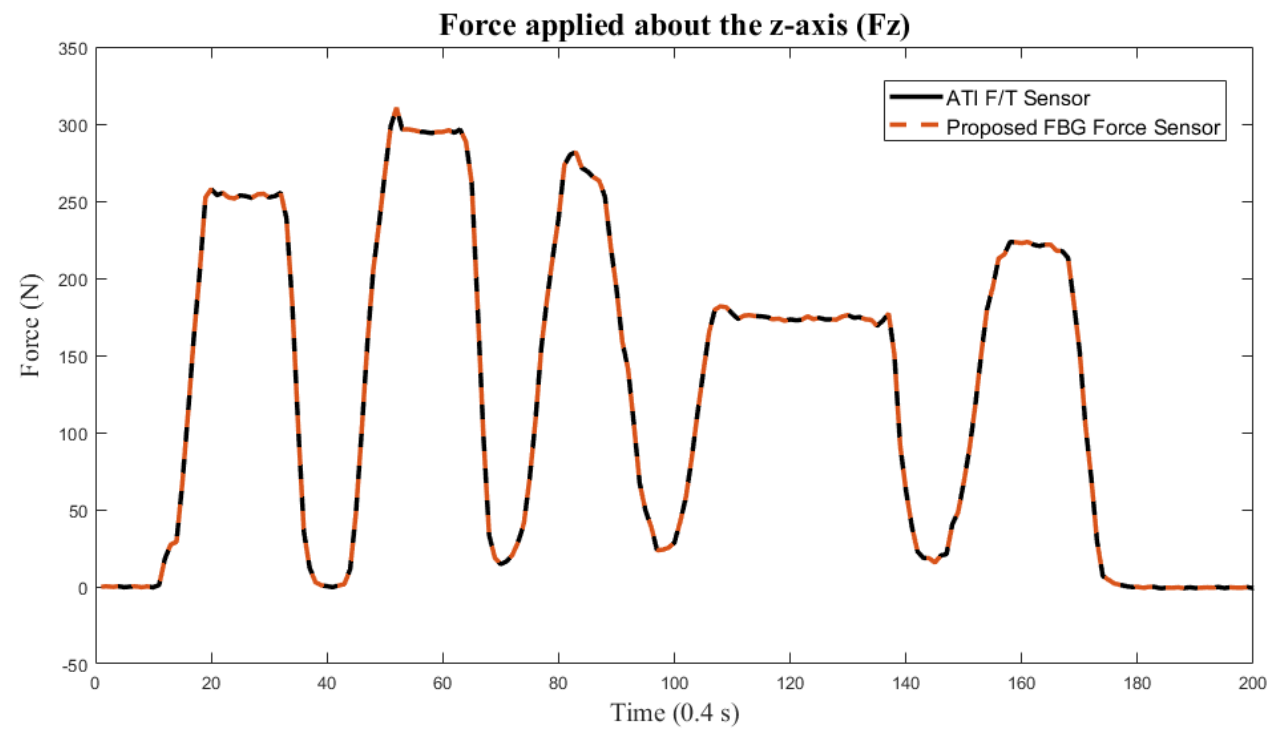

Figure 6.15: The performance of the fabricated FBG-based normal force sensing element compared to the ATI 6-Axis force and torque senso when the novel LR-NARX model is used to characterize the sensor. The experiment results are perfectly matches the force reference.

In addition to the improvement in the curve fitting value, the computational time required for convergence to achieve an excellent force estimator was significantly reduced which resulted in a faster speed of prediction for the residuals. The speed of prediction of the proposed LR-NARX was increased by over $60 \%$ compared to the speed of prediction when a NARX model alone is used. This is attributed to the smaller number of iterations needed for the training process. In the prediction process, the number of the iterations is continuously increasing until there is no significant decrease in the errors. In the current proposed LR-NARX, the linear portion of sensor 
data (which is the largest) is not incorporated in the NARX model, only the nonlinear portion of the sensor data. Therefore, the number of iterations in the LR-NARX is expected to be less than those needed in the NARX model alone.

The experimental results have confirmed our hypothesis to number of iterations in case of the LR-NARX estimation model: 61 iterations for the LR-NARX model and 214 iterations for the NARX model. Lower iterations results in a higher prediction speed with a higher precision of force measure in real-time.

Figure 6.17 and Figure 6.18 shows the performance of the shear force sensing element compared to the ATI 6-axis force and torque sensor. The coefficient of determination is found to be $98 \%$ when the proposed LR-NARX model is applied, which is an improvement compared to the NARX model alone with $97.5 \%$ and the linear least squares regression alone with 92\%. Table 6.2 summarizes the results obtained from the fabricated shear force sening element

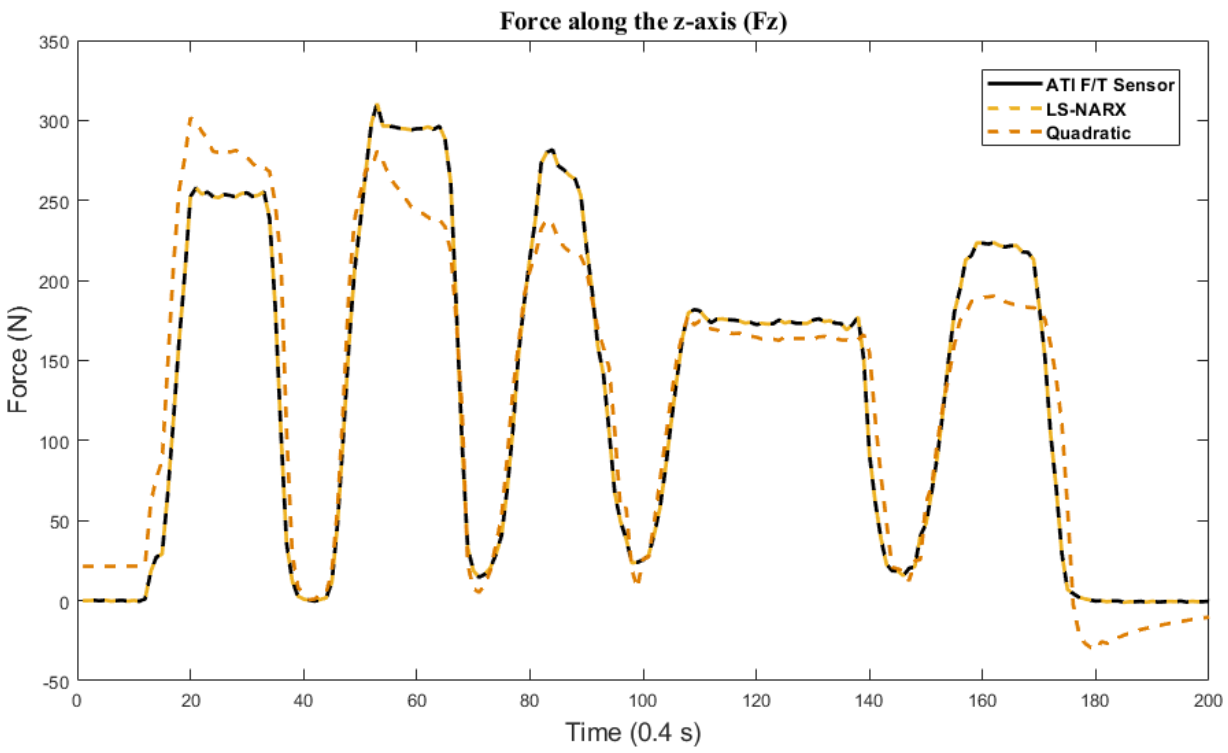

Figure 6.16: A comparison between the performance of the novel LR-NARX and the performance of the static quadratic model. 


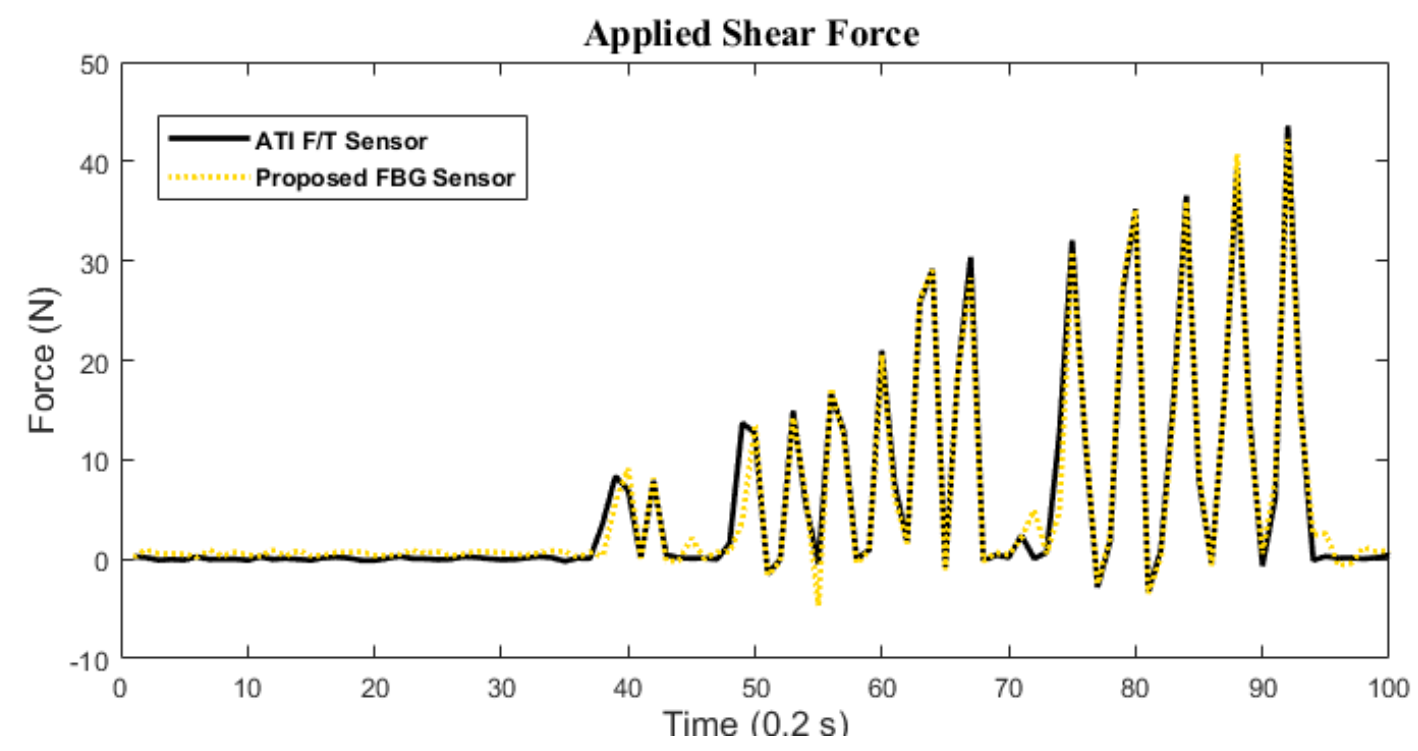

Figure 6.17: The performance of the fabricated FBG-based shear force sensing element compared to the ATI 6-Axis force and torque sensor.

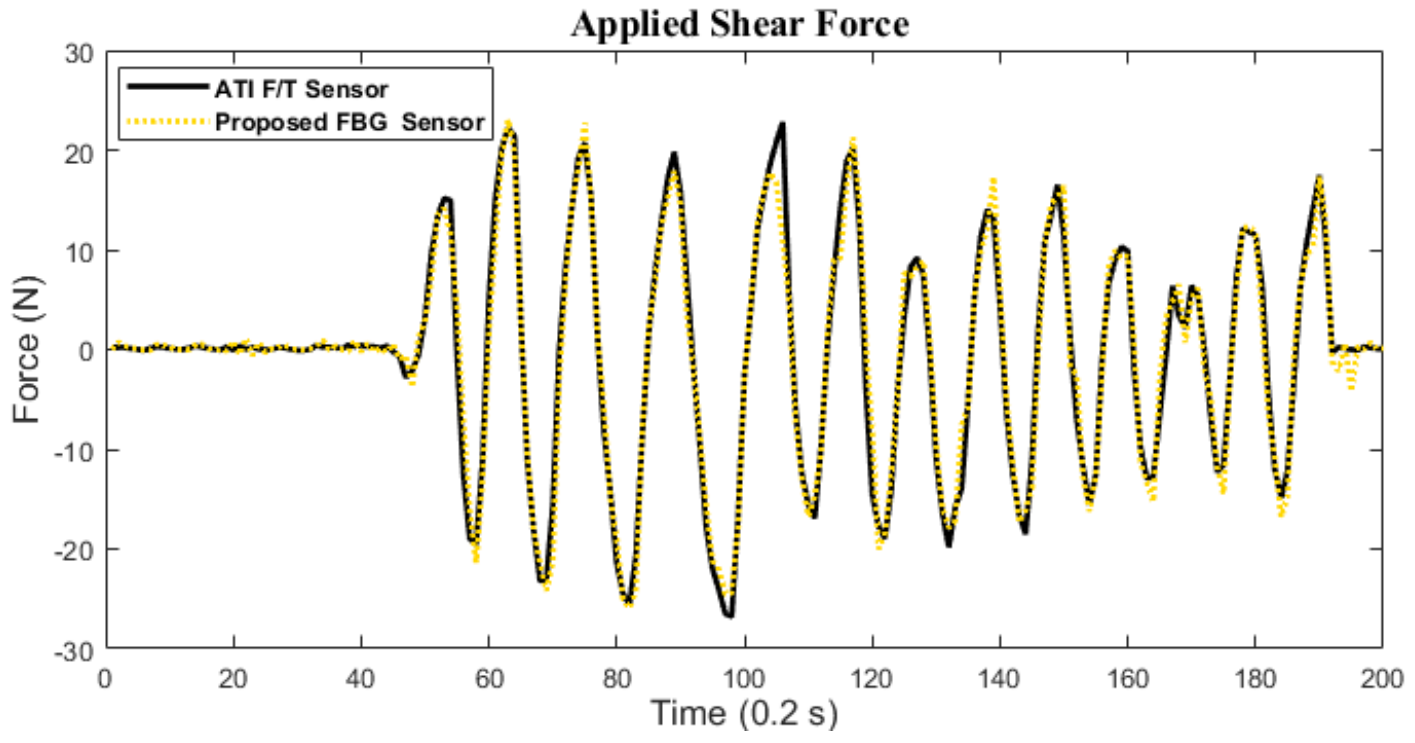

Figure 6.18: The experimental results of the fabricated FBG-based shear force sensing element compared to the ATI 6-Axis force and torque sensor. The results matches the force reference. 
Table 6.1: A summary of the experimental results: A comparison between the three proposed estimation models, LR-NARX, NARX alone and the linear least squares method alone in terms of the speed of prediction and the goodness of the fitting value.

\begin{tabular}{ccc}
\hline Type of model & Speed of prediction (obs/sec) & Fitting value $\left(R^{2} \%\right)$ \\
\hline \hline LR-NARX & 8,200 & 100 \\
\hline NARX alone & 2,050 & 99.30 \\
\hline LR alone & 49,000 & 93 \\
\hline \hline
\end{tabular}

Table 6.2: A summary of the experimental results of shear force sensor: A comparison between the three proposed estimation models, LR-NARX, NARX alone and LR alone.

\begin{tabular}{ccc}
\hline Type of model & Speed of prediction (obs/sec) & Fitting value $\left(R^{2} \%\right)$ \\
\hline \hline LR-NARX & 9,316 & 98 \\
\hline NARX alone & 2,329 & 97.5 \\
\hline LR alone & 48,000 & 92 \\
\hline \hline
\end{tabular}

Overall, the proposed FBG-based force sensor exhibits good results even with the linear least squares model. This is because the major portion of the sensor data is linear, providing a good curve fitting value of $93 \%$ when a linear least squares model is used to characterize the sensor. This is predictable as the source of the nonlinearity errors caused by the compliance of the material involved in the sensor's manufacturing process. The stiffness of the compliant material (Rubber) used can be adjusted by changing the mixing ratio of the curing agent and the rubber, the nonlinearity of the sensor can be controlled by increasing the stiffness of the rubber (spring constant $\mathrm{k}$ ) of the sensor system. This is a function of application and can be adapted by using a different material in the sensor, achieving a generic form of a sensor whose specification can be adjusted based on the requirements of the intended application. 


\subsubsection{Spline Regression for Sensor characterization}

The fabricated FBG-based force sensor has a confirmed linear relationship between the applied force and the generated wavelength change when the maximum force is within a certain degree of flexibilty (deflection), which was measured according to a standard hardness measurement and its corresponding Young's modulus, provided by the manufacturer. The Durometer Shore Hardness Scale uses a shore durometer (a device) for measuring the hardness of material. In this work, a silicon rubber with a Young's modulus of 2.159 $\mathrm{MPa}$ and durometer shore of $60 \mathrm{~A}$ has been selected. In the case where a high deflection force sensor is required using silicon rubber with a Young's modulus between 0.84 MPa and 1.70 MPa, we propose another solution using a cubic spline function. A cubic spline is effective in fitting nonlinear relationships. Spline models have been used to characterize different classes of sensors such as pressure sensitive resistors [281, 282], where the spline function was used to describe the nonlinear relationship between pressure and the electric resistance.

Figure 6.19 and Figure 6.20 demonstrate the performance of the proposed cubic spline estimation model alongside a linear least squares method. Both models are compared to the actual shear force data obtained from the ATI 6-axis force and torque sensor. As it can be seen in Figure 6.20, the proposed shear force sensing element is able to detect the magnitude and the direction of the applied shear force. In this nonlinear calibration, the acquired data is used to describe the relationship between the applied force and the generated variation in the light wavelength, producing a curve-fitting estimation model. The sensor output data is then used as input to the achieved spline model and the output of this model is the estimated force.

The proposed cubic spline estimation model produced a fitting value of $97.78 \%$ with 


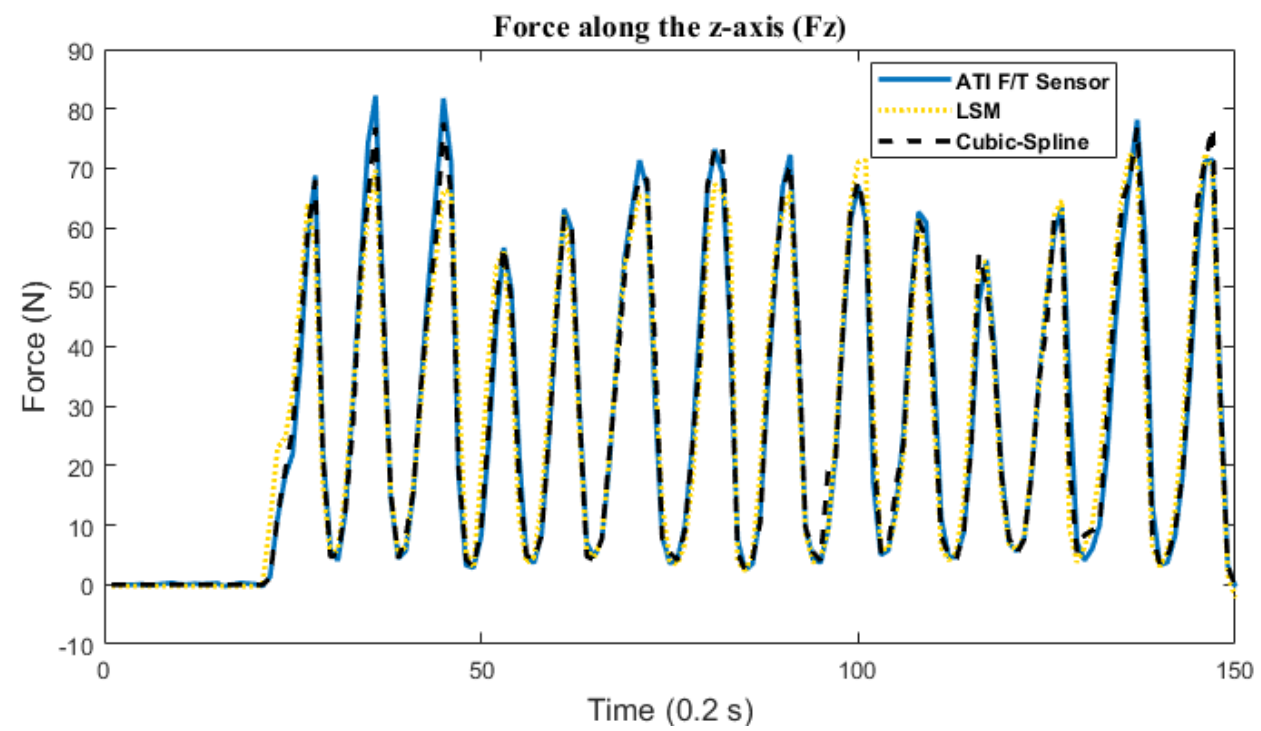

Figure 6.19: A comparison between cubic spline estimation model and a simple linear least squares method when a normal force is applied along the $\mathrm{z}$-axis

RMSE of $3.76 \mathrm{~N}$. The normalized RMSE\% is $4.57 \%$. Therefore, this is another possible estimation model to characterize the sensor which indicates the ability of the spline model to capture the nonlinear behaviour of the flexible material and provide a more accurate force estimate.

As it can be seen in Figure 6.21, the sensor output response (in wavelength) is mostly linearly related to its corresponding input applied force, as seen in Figure 6.19; it indicates that the largest portion of the sensor output data is linear. The proposed cubic spline model is able to fully capture both the linear and the nonlinear portions of the sensor output data.

This work discussed a generic form of a customizable sensor whose specifications can be adjusted based on the application requirements, such as in human-machine interface and gait measurements. The measuring range of the sensor, the accuracy, sensitivity and the resolution can be all adjusted since they all rely on the generated deflections and strains which are all controlled by stiffness/flexibility of the material 


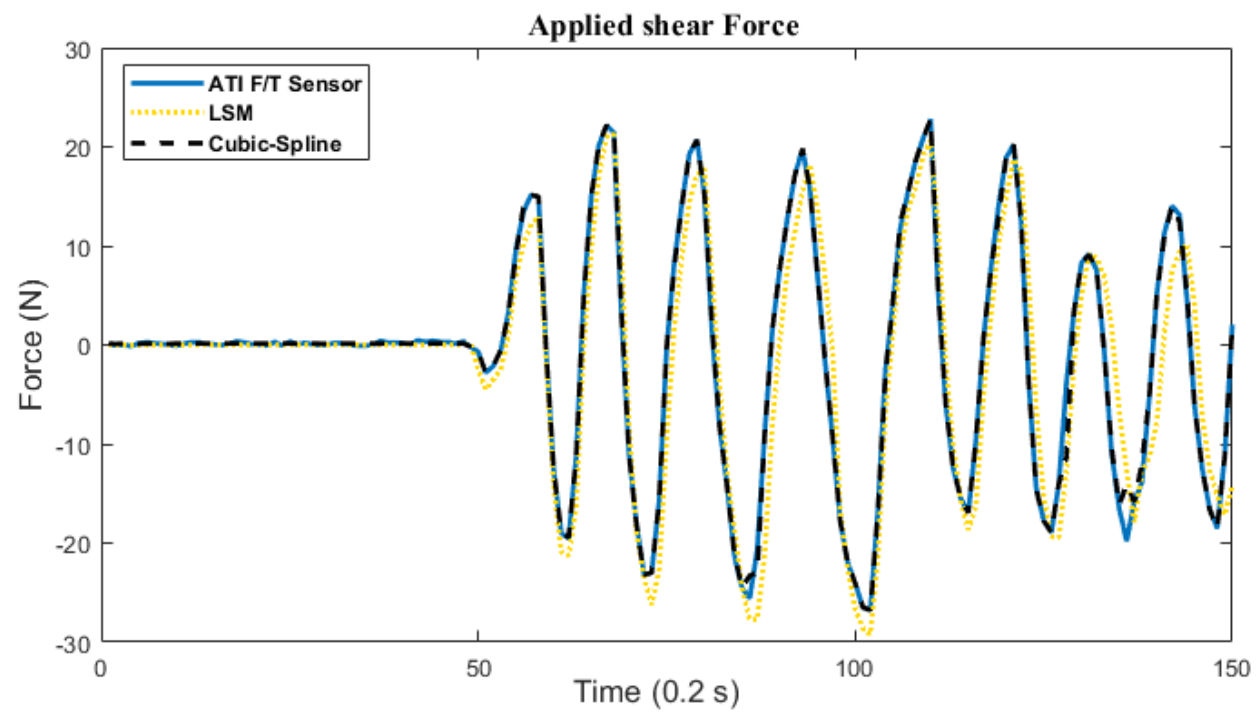

Figure 6.20: The experimental results of shear force sensing element in a comparison between the linear calibration using least squares method and a cubic spline estimation model.

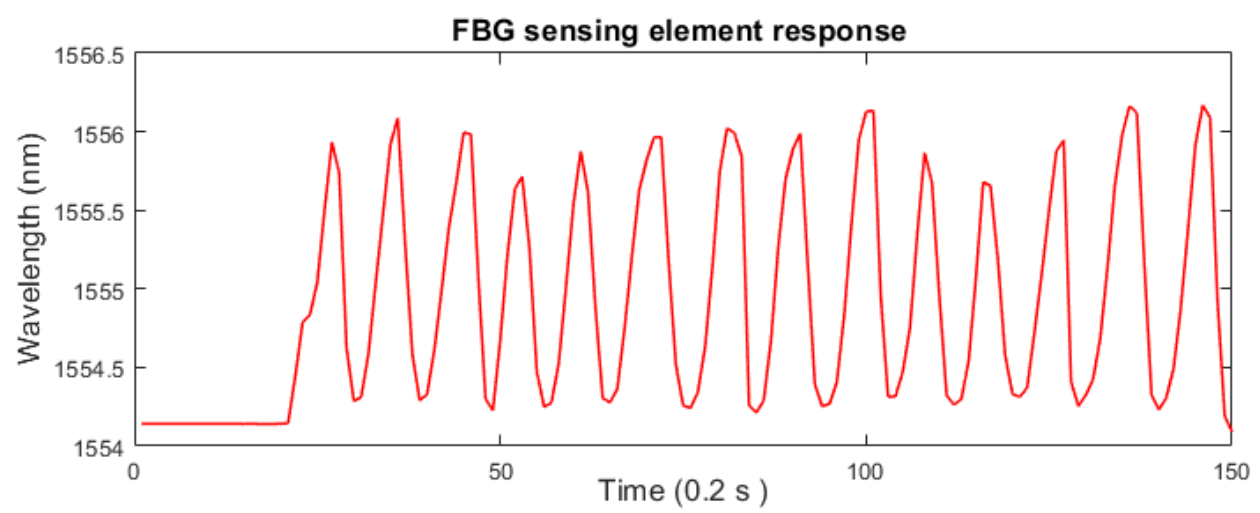

Figure 6.21: The response of the FBG sensing element. The generated wavelength variation due to the applied force 
(rubber) used in building the sensor. The stiffness/flexibility of the material can be adjusted by changing the mixing ratio of the rubber and the curing agent during the sensor's manufacturing process. In doing so, a higher amount of force can be applied to generate the required amount of deflection and strain along the material where the FBG sensor is embedded. This change in mechanical property will also increase or decrease the measuring range depending on whether the stiffness is increased or the flexibility is increased.

The resolution of the developed sensor is the smallest variation in mechanical deformation input (the applied force) that can generate a change in the output wavelength signal, which was found to be $0.09675 \mathrm{~N}$, suitable for the intended application. The sensitivity of the sensor is $0.054 \mathrm{~N} / \mathrm{nm}$ with a small repeatability error of $0.019 \%$. The hysteresis of the sensor was characterized using the proposed novel calibration methodology and was found to be insignificant: $2.8 \%$ of the full scale. The hysteresis is mostly attributed to compliant material used in the sensor development process which can be further decrease by increasing the stiffness of the material used. The nonlinear dynamic modelling proposed in this work was effective in characterizing the hysteresis.

\subsection{Conclusion}

In this chapter, compliant, small sized, lightweight, fiber Bragg gratings-based, normal and shear force sensing elements have been designed, developed and characterized. The performance of the sensing elements was investigated and evaluated through several experiments using static and dynamic loading tests. Both normal and shear force sensing elements have shown the ability to accurately measure the applied force 
with a possibility to adjust the force sensing range based on the desired intended application. To enhance the sensitivity of the sensor the optical fiber was pre-stretched and glued at both sides of the sensing body enabling shear force measurements in both directions using only one optical fiber. Due to its compliance, non-electrical conductivity, immunity to electromagnetic interference and its wide force measurement range, the sensing elements exhibit excellent biocompatibility that can be used for several biomechanical measurements such as in human-machine interface, total ground reaction force $(\mathrm{GRF})$, and any other applications where flexibility of the sensor is desirable, and rigidity is an issue.

Several force estimation models were applied including a simple linear least squares method which has shown a precise force estimation. However, in the case where a large amount of force is applied (generating a large deflection), nonlinear behaviour was observed. Therefore, a novel effective calibration methodology was applied, obtaining accurate results and high sensing performance. The calibration procedure incorporates two estimation models: a nonlinear model based on the nonlinear autoregressive exogenous (NARX) which has been used as an error estimator; and a linear least squares model to estimate the force data. The sum of both models generates the proposed calibration system that works effectively in real-time.

The proposed novel calibration procedure is able to obtain higher accuracy than the linear least squares calibration tool due to its ability to capture the nonlinearity attributed to the high compliance and large deflection. 


\title{
Chapter 7
}

\section{Novel Calibration Methodologies for \\ Compliant Multi-Axis Force/Torque}

\section{Sensors}

\begin{abstract}
In this chapter, we present a number of novel calibration methodologies for compliant, multi-axis, fiber-optic based force/torque sensors and evaluate the performance of these methods in real-time experiments with our custom designed sensors. These methods address the challenges arising from the important dynamic behaviour of a compliant sensor and the nonlinearities in force-deflection relationship. This chapter also investigates compliant sensor performance against its response characteristics, such as the impact of compliance on the sensor's bandwidth using dynamic modelling and identification process. A powerful hysteresis compensation solution using a
\end{abstract}


dynamic estimation model is also presented. Furthermore,we propose and apply a new calibration strategy building on the combination of a linear dynamic model combined with a static nonlinear model. This includes a state space model with Gaussian Process Regression model named State Space Gaussian Process Regression (SSGPR). The results achieved from the proposed calibration method have revealed an improvement from an R-squared value of $93.86 \%$ to $100 \%$ when compared to a data obtained using a linear dynamic model.

\subsection{Introduction}

Compliant, multi-axis, force/torque sensors play a major role in the health care industry and in biomechanics measurements. Since they can provide the whole force and torque information of any structural joint, these six-axis, force/torque sensors have been extensively used in a broad range of applications such as in robotics, human-machine interface, biomechanics and rehabilitation systems [207, 218, 283-286].

Although several careful considerations and ideas [211, 219, 220, 287] have gone into the design, development, and characterization of compliant, multi-axis, force/torque transducers, the challenges associated with the behaviour of compliant materials is unavoidable and problematic. The interference of the external environment, the error of the measurement system, and the crosstalk effects on each sensing element of the multi-axis transducers impose large uncertainties that need to be addressed before putting these transducers or sensors into application. These uncertainties create a complex nonlinear relationship between the generated output signal of the sensor and the applied force/torque. 
Compliant force and torque sensors in particular exhibit a nonlinear dynamic behaviour due to the large deflection and the viscoelastic property of the compliant materials. At this level, these compliant materials show a delay phenomenon between its output signals and its inputs. This is attributed to the complex mechanism in energy dissipation caused by its viscoelasticity. Therefore, the modelling of such devices and materials requires the ability to analyze the associated memory effects, ie. the hysteresis effects. In real feedback systems that use flexible actuators or sensors, hysteresis could potentially cause a severe limitation on the performance of the sensing device and systems, in terms of seeking precision, accuracy or stability. In order to enhance the measuring precision, it is required to calibrate the sensor for a real-world, practical relationship.

Traditional calibration methodologies involve parametric system identification procedure through a regression analysis based on presumed static models that have been extensively used in sensor calibrations. As one of the conventional calibration methodologies, the least squares optimization technique has been widely applied to calibrate multi-dimensional transducers [3, 288-292]. This method is effective and can be used for rigid, metal-based sensors, showing the ability to eliminate most of the crosstalk effects. It can also be used for compliant sensors where a small deflection is generated. However, in the case where high forces are applied or where a large deflection is produced on the compliant sensors, such methods cannot capture the associated nonlinearities since it is a fairly linear calibration approach; therefore, a proportional error is generated. More importantly, the hysteresis cannot be characterized using a static linear methods.

A finite element analysis (FEA) based calibration has been used in [195, 196, 219, 293-295]. However, such calibration methods rely on several assumptions, 
such as negligible deformation at the cross beams, isotropic elasticity and high precision alignment for each sensing element (which is required during bonding process and cannot be physically achieved). Artificial Neural Networks (ANN) and decision trees-based regressions are capable of predicting various nonlinear relationships which have been utilized to calibrate single and multi-dimensional force and torque sensors [2, 3, 221-223]. Nevertheless, artificial neural networks in most cases, have a few shortcomings: the need for large network scales and substantial amounts of training time. Moreover, the ANN cannot compensate for the hysteresis effects since what is needed is a nonlinear dynamic model while it can only provide a static nonlinear model.

In this chapter, we propose and investigate a number of different calibration methodologies that have confirmed their effectiveness in characterizing the hysteresis and the dynamic behaviour related to the compliant sensors and its materials. Also, we investigate the conventional linear calibration methods and prove that they show good performance for compliant sensors when a small deflection is generated.

Finally, a novel dynamic two-model based calibration methodology is proposed and used. The method is a mixture of a parametric knowledge-based estimation model, based on a dynamic linear state space (SS) estimation model to estimate the largest portion of sensor data which is linear and to capture the dynamic behaviour of the compliant sensor. The other part of the proposed calibration methodology is a non-parametric machine learning based Gaussian process regression model (GPR) to predict the errors and create one effective single calibration system, the SSGPR, that has confirmed its ability to characterize the complexity of the compliant force/torque sensors. 


\section{2 knowledge-Based (Parametric) Calibration Meth- odologies}

\subsubsection{Data-Driven Dynamic Modelling}

The bandwidth of compliant force/torque sensors is a crucial factor to evaluate sensor performance through its intended application. The flexibility of the fiber-optic based force/torque sensor is desirable in several biomechanical applications. However, this compliance can affect the bandwidth of the sensor: The higher the compliance, the larger the effects on the bandwidth will be; therefore, a dynamic modeling and system identification is essential to conduct an estimate of the best model based on measured input/output data.

A voltage-to-force transfer function and state space models were developed from the observed input and output data of our custom designed compliant sensor [2]. Figure 7.1 shows the performance of these models compared to the actual force.

Second order transfer functions were applied to estimate the normal force applied along the z-axis $(\mathrm{Fz})$, shear forces acting along the $\mathrm{x}$ - and $\mathrm{y}$-axis (Fx, Fy), torques applied about the $\mathrm{x}$ - and the $\mathrm{y}$-axis (Tx, Ty) representing tilting motions, and finally the torque applied about the z-axis $(\mathrm{Tz})$ representing spinning motions. This was performed to identify the fitting value, RMSE, and bandwidth.

Although the current state space model does not exhibit a more accurate result than the transfer function, it does however give a faster transient response and therefore higher bandwidth can be obtained when the third order of the state space model is 


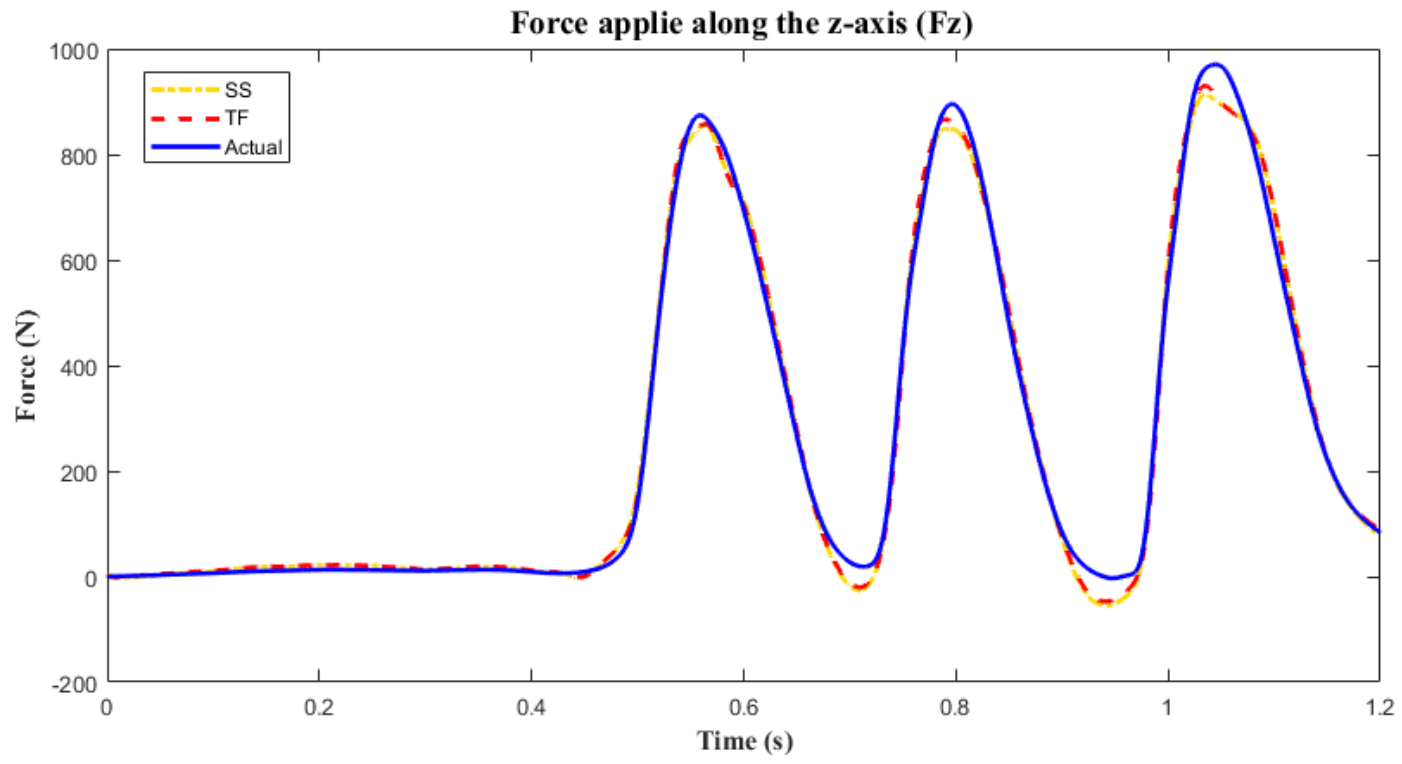

Figure 7.1: A comparison between the actual force and the estimated data using a transfer function model and a state space model

applied. The third order state space model has been identified as follows:

$$
\begin{aligned}
& \frac{d x}{d t}=A x(t)+B u(t)+K e(t) \\
& Y(t)=C x(t)+D u(t)+e(t)
\end{aligned}
$$

where

$$
A=\left[\begin{array}{ccc}
-71.56 & -324.6 & 110 \\
-255.5 & -865.8 & -216.4 \\
-215 & -598.7 & -318.2
\end{array}\right]
$$




$$
\begin{aligned}
B & =\left[\begin{array}{l}
-4.420 \\
-19.50 \\
-16.10
\end{array}\right] \times 10^{6} \\
C & =\left[\begin{array}{lll}
-578.6 & 691.4 & -678.5
\end{array}\right] \\
K & =\left[\begin{array}{l}
-1.123 \\
-4.955 \\
-4.092
\end{array}\right] \times 10^{4}, \quad D=0
\end{aligned}
$$

The third order state space model has a shorter rise time of only 0.001 second, whereas the rise time of the second order state space model has 0.004 second. The system overshoot is also decreased to 17.69 compared to the overshoot of second order state space model with 25.63. As a result, the bandwidth of the compliant sensing system was estimated using a transfer function and a state space model which was increased from $65 \mathrm{~Hz}$ to $230 \mathrm{~Hz}$ when a third order state space model is used. Table 7.1 summarizes a comparison between the three estimation models.

Table 7.1: A summary of the experimental results: A comparison between the three proposed estimation models, $2^{\text {nd }}$ order transfer function model, $2^{\text {nd }}$ order state space model and $3^{\text {rd }}$ order state space model in terms of the goodness of the fit, RMSE and Bandwidth.

\begin{tabular}{cccc}
\hline & $2^{\text {nd }}$ order TF & $2^{\text {nd }}$ order TF & $3^{\text {rd }}$ order SS \\
\hline Fitting Value & $93.86 \%$ & $93.86 \%$ & $93.30 \%$ \\
\hline RMSE & $1.92 \%$ & $2.01 \%$ & $2.14 \%$ \\
\hline Bandwidth & $65 \mathrm{~Hz}$ & $65 \mathrm{~Hz}$ & $230 \mathrm{~Hz}$ \\
\hline
\end{tabular}




\subsubsection{Hysteresis Compensation}

Compliant materials exhibit large deformation and deflections in response to the external forces. These type of materials have shown their importance in the development of various devices for sensing and actuation purposes. Particularly in applications such as biomechanics, human-robot interaction and assistive devices where compliant transducers are desired. In this chapter, we propose a dynamic calibration approach that is able to effectively reduce the hysteresis effects.

A second order transfer function estimation model is applied which is capable of significantly decreasing the effect of hysteresis by $95.05 \%$, generated by the compliant materials used in the sensor. Figure 7.2 shows the ability of the proposed transfer function model to characterize the hysteresis, indicating the effectiveness of the dynamic modelling and system identification procedure in this compliant sensor characterization, as well as the third order state space model in removing the hysteresis effect.

\subsubsection{Static Calibration}

In order to better investigate sensor performance and evaluate the bandwidth of the proposed sensor, new types of experiments were conducted using the experimental setup shown in Figure 7.3. The compliant force/torque sensor is mounted onto the ATI 6-axis $\mathrm{F} / \mathrm{T}$ sensor, then different amounts of forces at high frequencies are applied. This was done by hitting the sensor as fast as possible while data is recorded from both the reference sensor and the compliant sensor at a sampling rate of $1 \mathrm{kHz}$. This experimental procedure is used due to the lack of high frequency machine-applied force 

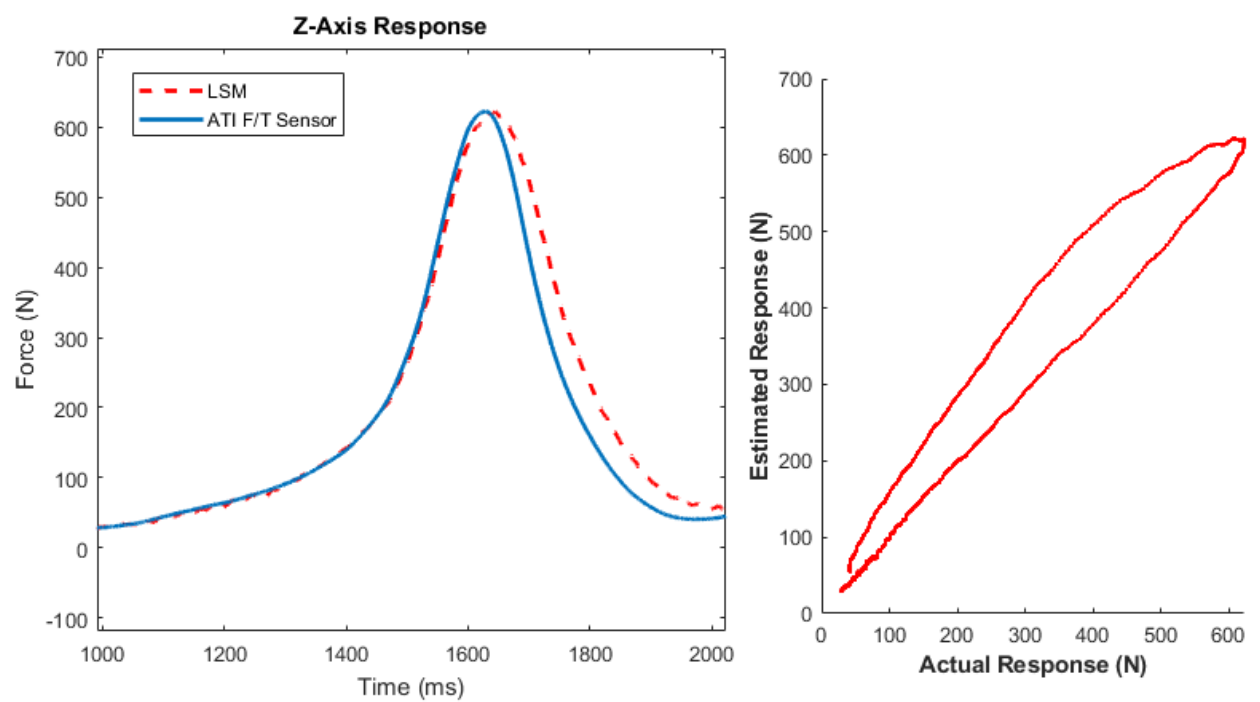

(a)
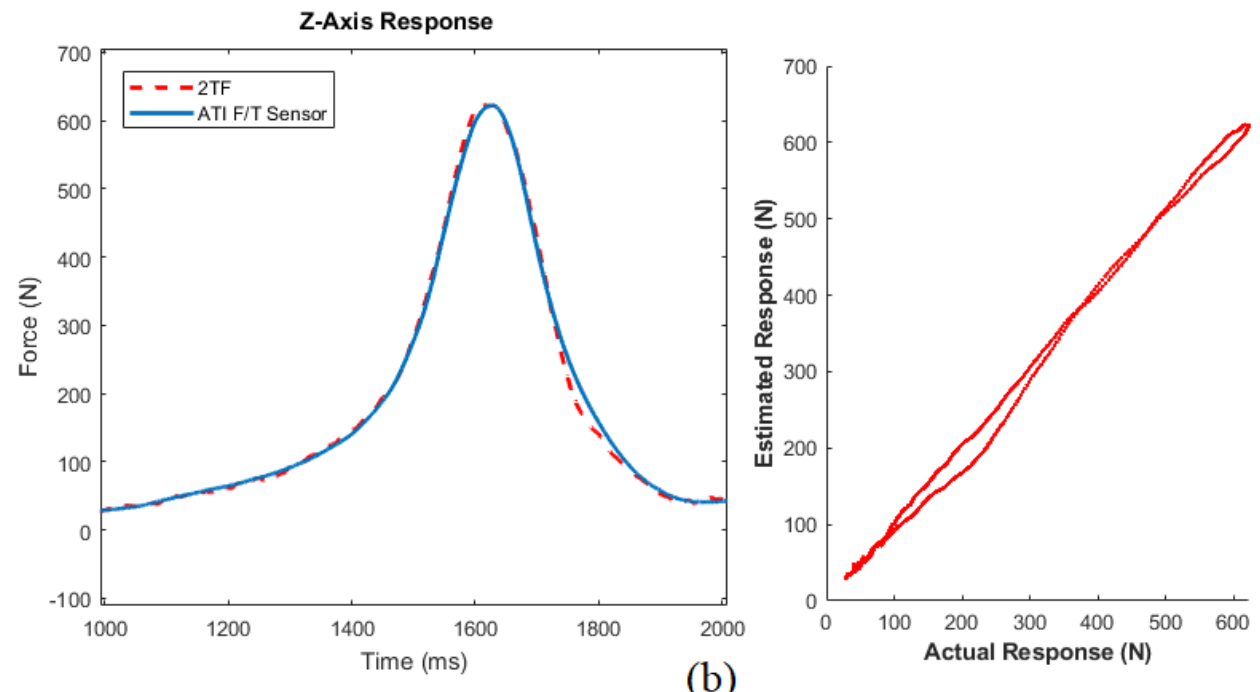

(b)

Figure 7.2: Performance of the proposed dynamic models and their ability in characterizing the dynamic behaviours of the compliant force/torque sensors. 
testing devices. The commercially available MTS Bionix Servo-hydraulic Test System provides a maximum frequency of only $2 \mathrm{~Hz}$, which is not satisfactory.

Data is recorded from each axis while the proposed sensor is being hit as shown in Figure 7.3, and sensor data is then compared to an actual force measurement obtained from the reference sensor, ie. the ATI 6-axis $\mathrm{F} / \mathrm{T}$ sensor. In these experiments, the sensor is characterized by applying a linear least squares method (LSM) as a multi-input and multi-output linear optimization procedure. Figure 7.4 (a) demonstrates the response of the sensor due to the normal force applied along the z-axis in comparison to the ATI 6-axis F/T sensor. Figure 7.4 (b) is a closer look of the same plot to show that the experimental and actual data overlap exactly, meaning the compliant sensor exhibits a very similar performance (fast response) compared to the reference. The root means square error (RMSE) is $6.21 \times 10^{-8} \mathrm{~N}$, which is very small, showing negligible error.

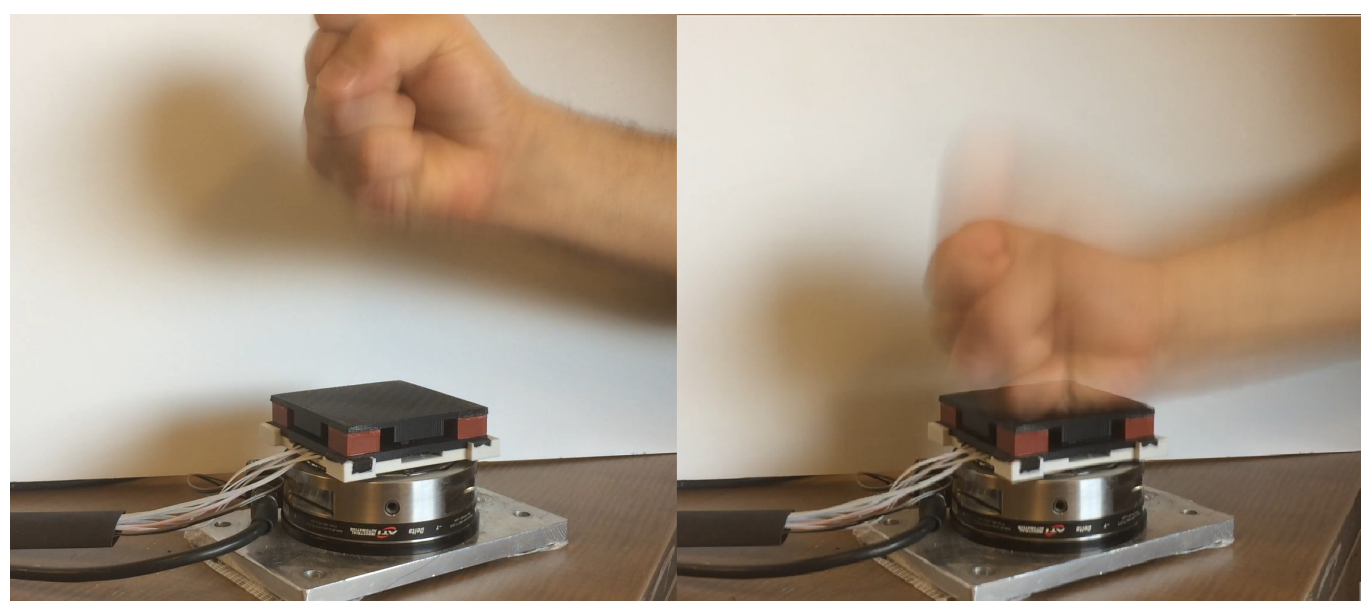

Figure 7.3: The experimental setup of the bandwidth test. A high frequency force is applied by hitting the propose sensor continuously generating high speed force.

Figure 7.5 and Figure 7.6 show the response of the shear force sensing elements due to the applied force along the $\mathrm{x}$ - and the $\mathrm{y}$-axis, respectively. The external force is arbitrarily applied by hand during these experiments. 
Figure 7.5 compares the dynamic response of the $\mathrm{x}$-axis sensing element of the compliant sensor to the reference force data which also exhibits a very accurate result with an RMSE of $3.25 \times 10^{-8} \mathrm{~N}$. The coefficient of determination or goodness of fit (R-squared $R_{2} \%$ ) is $100 \%$. Similarly, Figure 7.6 shows a comparison between the measured and the estimated data of the other shear force component (Fy). The estimated shear force obtained from the sensor matches the actual force, resulting in a RMSE of $3.23 \times 10^{-8} \mathrm{~N}$ and an R-squared of $100 \%$.

The proposed experimental procedure also influences the rest of the torque sensing elements, generating torques about the $\mathrm{x}-, \mathrm{y}$ - and $\mathrm{z}$-axis $(\mathrm{Tx}, \mathrm{Ty}, \mathrm{Tz})$ in a range between 0 N.mm to 450 N.mm. In terms of the torque applied about the $\mathrm{x}$-axis, the sensor produces a RMSE of $2.45 \times 10^{-6} \mathrm{~N}$.mm and an R-squared of $100 \%$. These results are shown in Figure 7.7.

For the torque applied about the $\mathrm{y}$-axis (Ty), and the torque applied about the z-axis (Tz), the sensor exhibits a high quality performance, generating very small RMSE of $2.93 \times 10^{-6}$ N.mm and $2.78 \times 10^{-6}$ N.mm with R-squared of $100 \%$ and $100 \%$ for the Ty and Tz sensing elements, respectively. Figure 7.8 and Figure 7.9 show the performance of each torque sensing element of $\mathrm{Ty}$ and $\mathrm{Tz}$ when a linear least squares method is used.

Although the torque range is relatively small in this particular test (which was expected to give a poor signal to noise ratio), the sensor is able to capture low magnitudes of force and torque with high accuracy. This means that the sensor produces very little noise, and provides high precision measurements. The bandwidth in this test reached to about $60 \mathrm{~Hz}$ and the compliant sensor data still matched the actual force. However, the bandwidth of this sensor can be adjusted by increasing or 


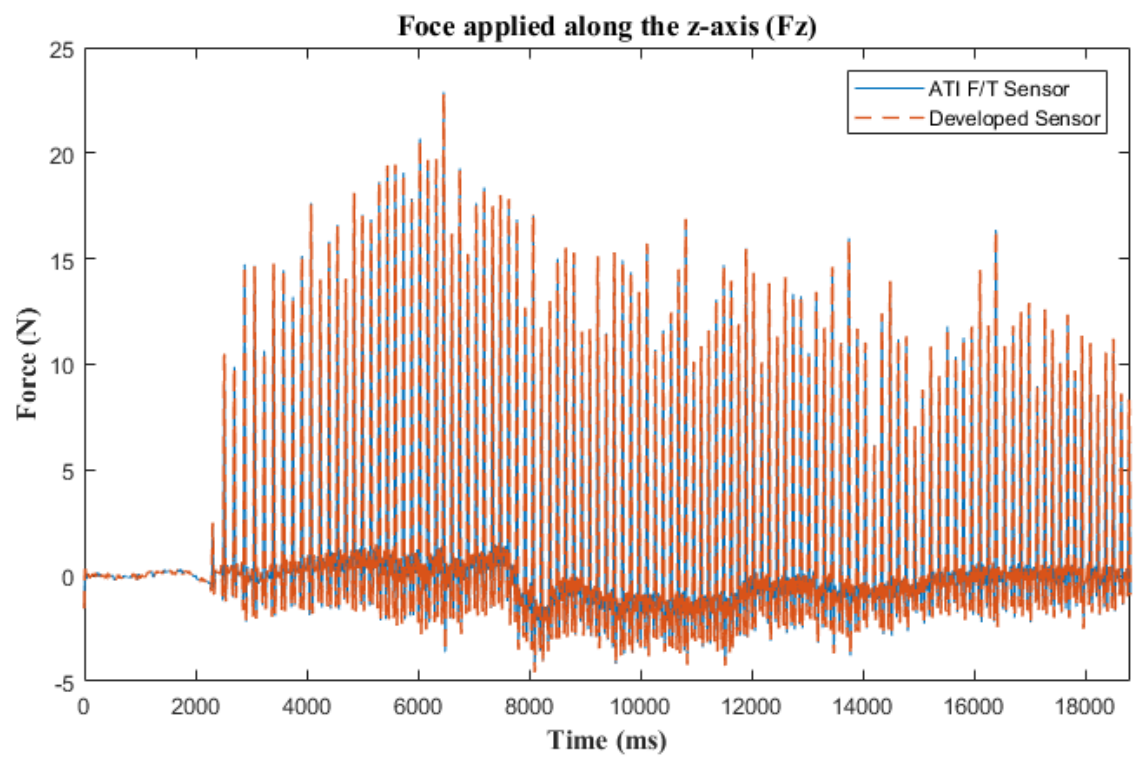

(a)

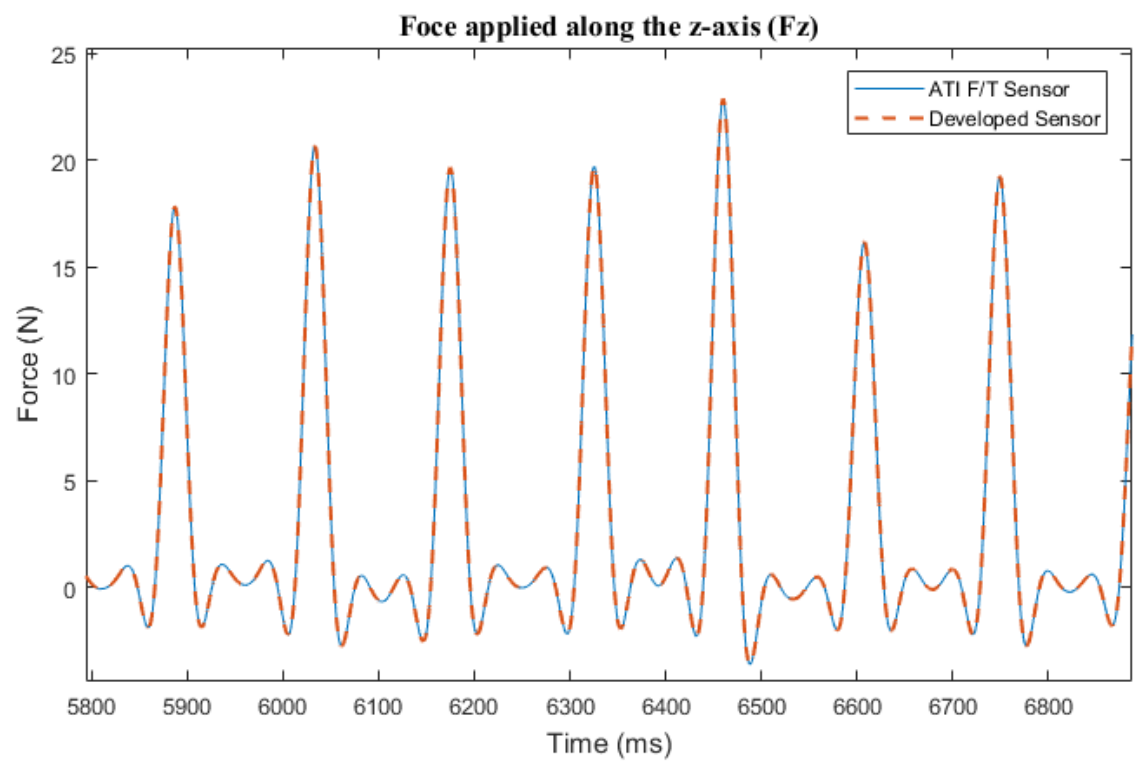

(b)

Figure 7.4: The experimental results of the proposed sensor. (a) and (b) the performance of the Fz sensing element under a higher frequency force of the applied normal force in a comparison to the acquired actual data from the ATI 6-axis $\mathrm{F} / \mathrm{T}$ sensor when the linear least squares approach is used. 


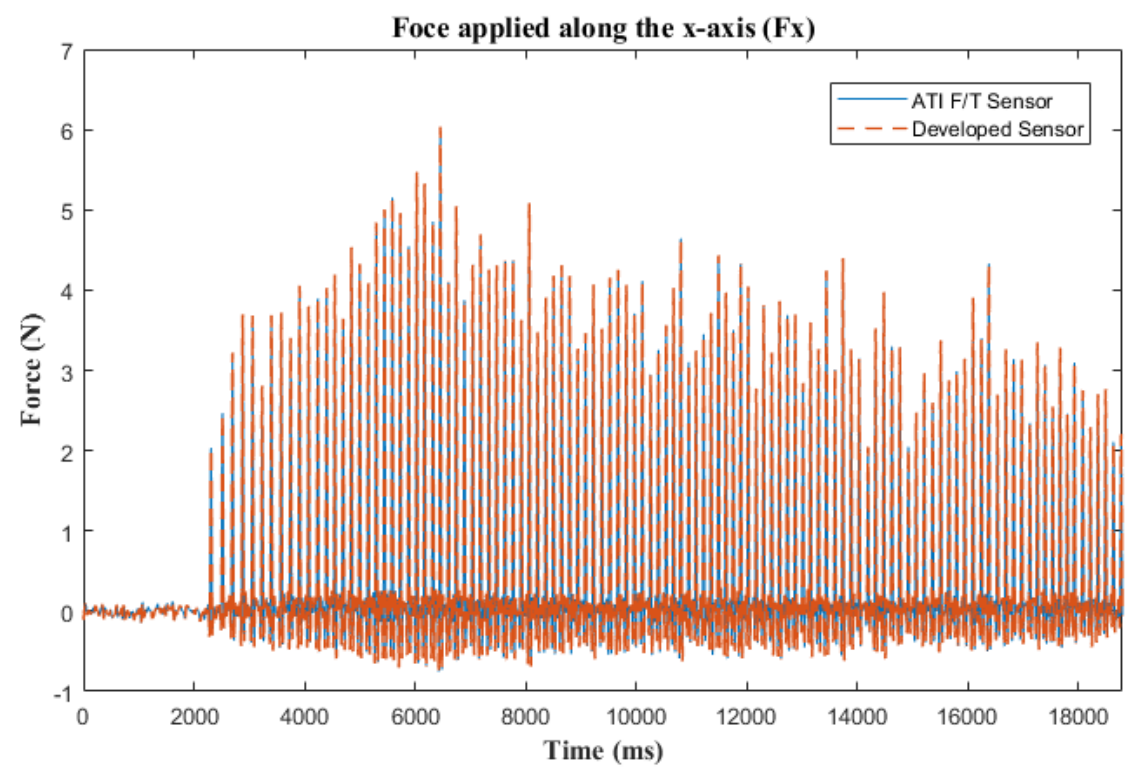

Figure 7.5: Compliant force and torque sensor response due to the applied shear force along the $\mathrm{x}$-axis using least squares optimization.

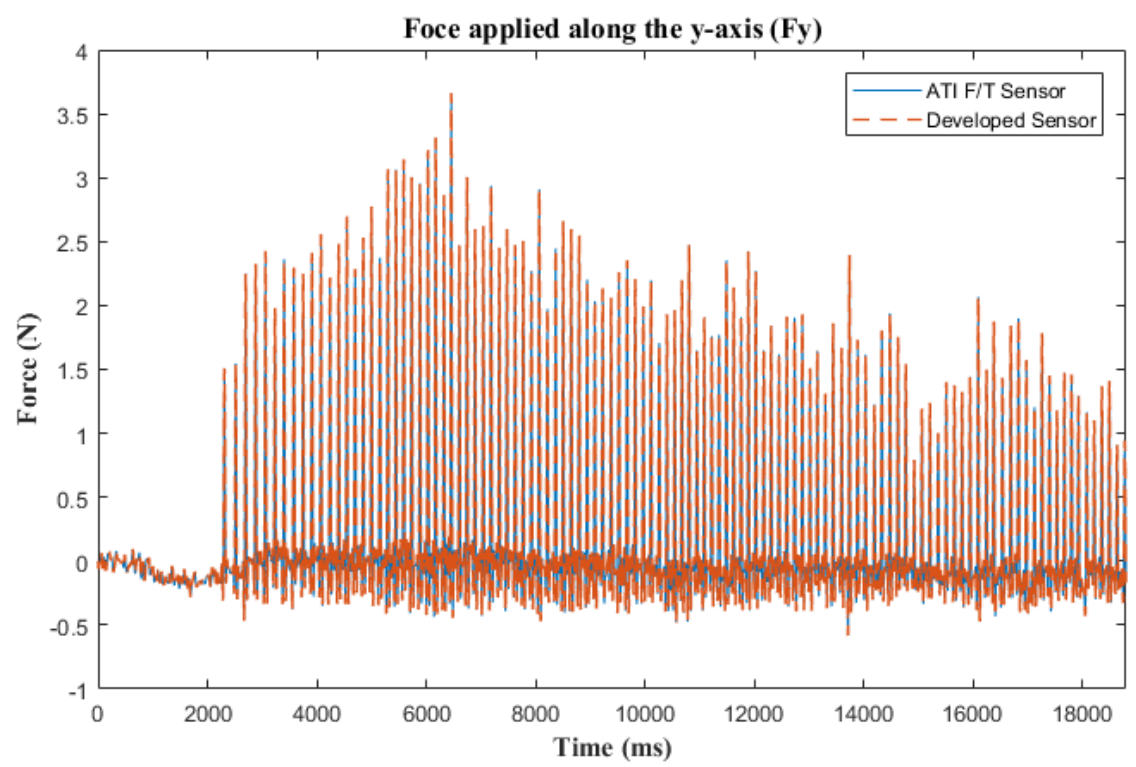

Figure 7.6: Sensor response due to the shear force applied along the y-axis. 
decreasing the elasticity of the compliant material used. The measuring range can also be adjusted using the same previously mentioned changes in material property. The rigidity can not affect the sensitivity of the sensor very much, since the optical sensor (LTV) exhibits very high sensitivity.

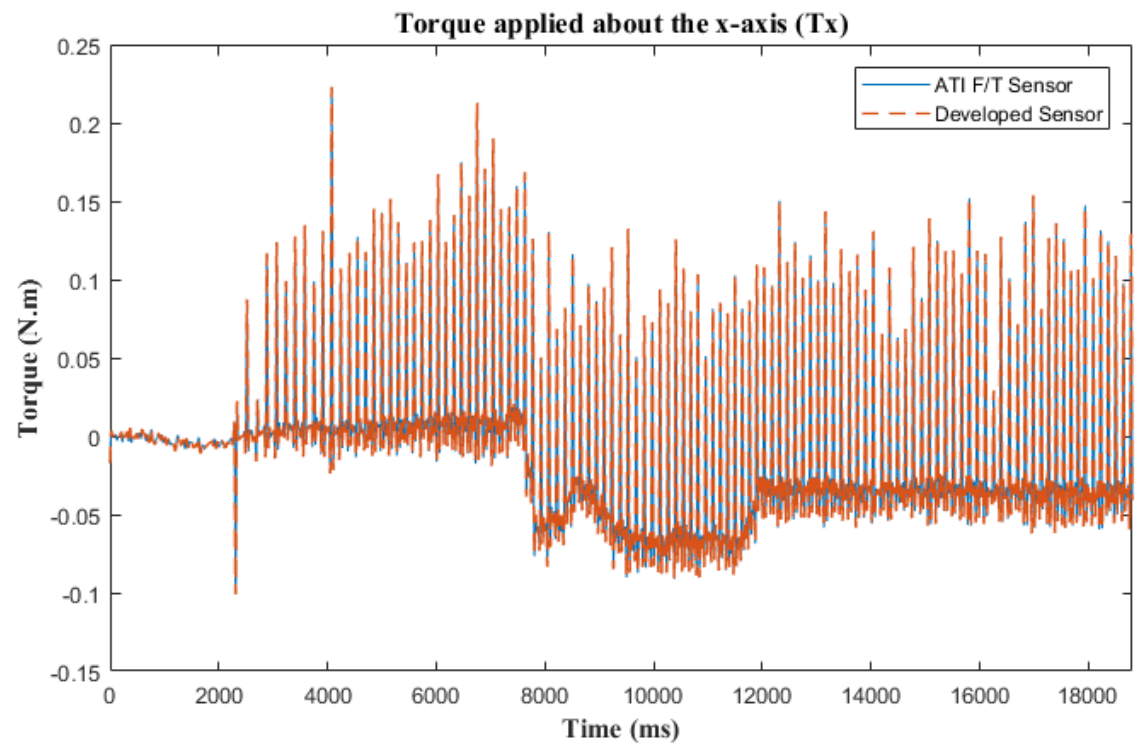

Figure 7.7: The experimental results when a linear least squares calibration is used to characterize the compliant force and torque sensor. This is a torque applied about the $\mathrm{x}$-axis.

\subsection{Non-Parametric Machine Learning Based Calibr- ation}

Precise estimation models for sensing systems are crucial in several applications. For complex systems such as compliant sensors that are designed for human robot interface, automated rehabilitation and surgical robotic hands and other biomechanical applications, it is not straightforward to sufficiently model these systems since they require smart prediction techniques. Modern model learning methods have the 


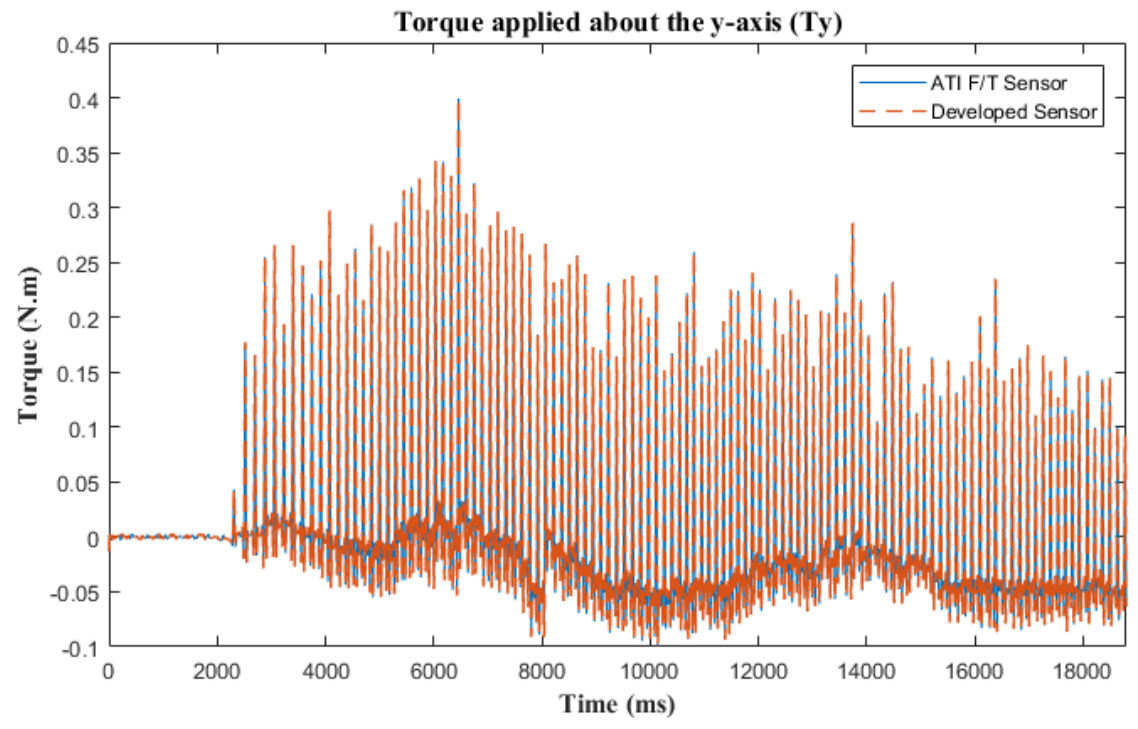

Figure 7.8: A Comparison between the performance of the compliant force and torque sensor calibrated using the simple linear least squares method and the actual torque data applied about the $\mathrm{y}$-axis.

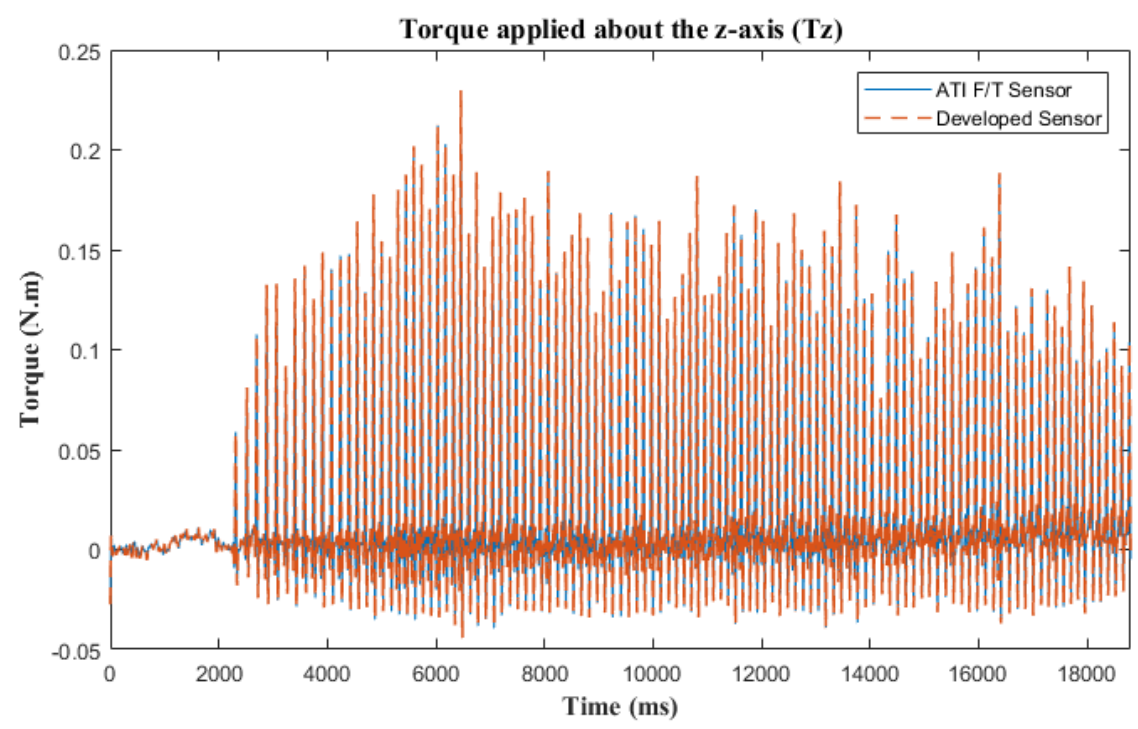

Figure 7.9: compliant force and torque sensor response due to the torque applied about the $\mathrm{z}$-axis in a comparison to the actual torque obtained from the ATI 6-axis force and torque sensor. 
capability to of efficiently characterizing such complex systems, providing excellent solution and a valuable alternative. Non-parametric machine learning-based methods can approximate any linear or nonlinear function relying on the input and the output data to characterize the relationship of the sensing device, regardless of any prior knowledge of the system.

However, in some cases of real-time applications, online machine learning modelling, experiences technical and regression challenges. The prediction speed should be relatively high enough based on the application requirements. Also, machine learning models must have the ability to handle large amounts of data sets. Such methods can be used for sensors that have highly nonlinear behaviour and for complex systems that cannot be mathematically modelled. Such methods could also be used for systems that cannot be fully described using knowledge-based models, such as compliant sensors that generate large deflections..

In this section, we propose Gaussian process regression models (GPR) and show their fitting performance and results. Three different Gaussian process regression models are used: Rational-Quadratic GPR, Squared-Exponential GPR and Exponential GPR. A comparison between the performance of each estimation model is investigated by applying a high force range to the compliant sensor, at which point the effects of dynamic behaviour are generated as a result of the force-to-deflection nonlinear relationship. A force with dynamic range of $1000 \mathrm{~N}$ is applied on the top surface of the sensor while data is continuously recorded.

The three GPR-based models' results are depicted in Figure 7.10. The Squared-Exponential GPR gives R-squared of $100 \%$ with RMSE of $1.86 \%$. This model has a satisfactory speed of prediction of $10,000 \mathrm{obs} / \mathrm{sec}$ (observation/second). On the 
other hand, Rational-Quadratic GPR exhibits a relatively higher estimation speed of $12,000 \mathrm{obs} / \mathrm{sec}$ with R-squared of $100 \%$ and RMSE of $1.87 \%$. The Exponential GPR estimation model has slightly less accurate results, generating RMSE of $2.3 \%$ with R-squared of $99 \%$. However, the model exhibits the highest speed of prediction of $19,000 \mathrm{obs} / \mathrm{sec}$.

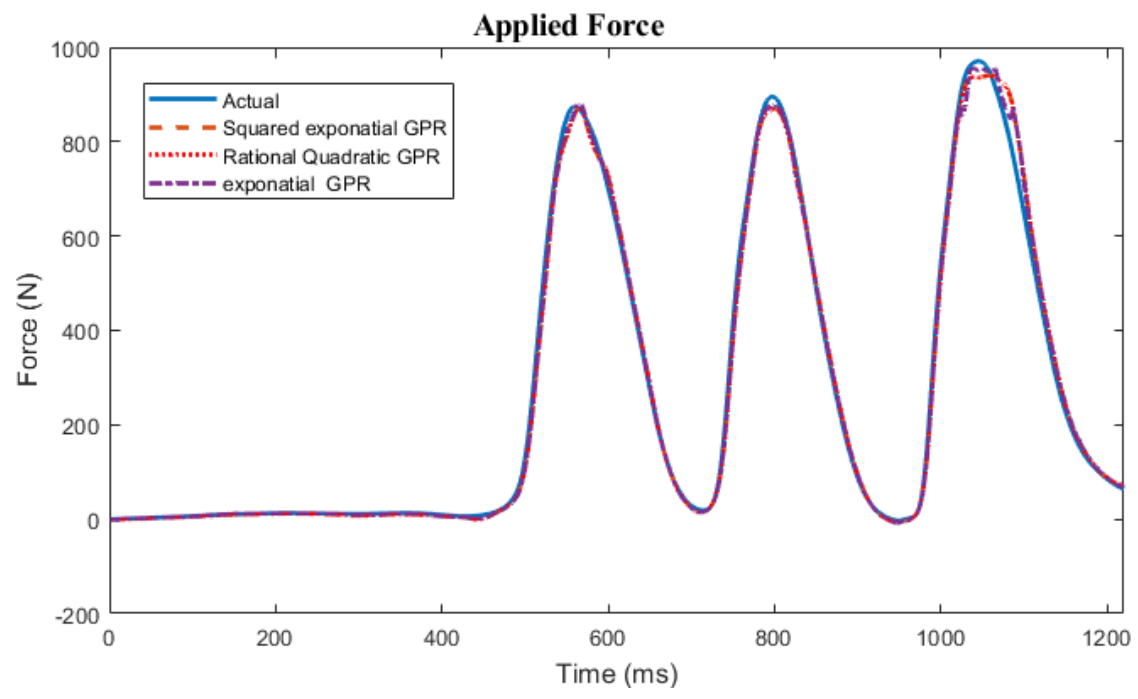

Figure 7.10: The performance of the proposed Gaussian process regression models in comparison to the applied actual force.

Table 7.2 summarizes a comparison between the proposed estimation models.

Table 7.2: A summary of the experimental results: A comparison between the three proposed Gaussian process regression models (GPR) estimation models, Rational-Quadratic GPR, Squared-Exponential GPR and Exponential GPR in terms of the speed of prediction and the goodness of the fitting value as well as the RMSE.

\begin{tabular}{cccc}
\hline Model & $R^{2}$ & Estimation Speed & RMSE \\
\hline Rational-Quadratic GPR & $100 \%$ & $12,000 \mathrm{obs} / \mathrm{sec}$ & $1.86 \%$ \\
\hline Squared-Exponential GPR & $100 \%$ & $10,000 \mathrm{obs} / \mathrm{sec}$ & $1.87 \%$ \\
\hline Exponential GPR & $99 \%$ & $19,000 \mathrm{obs} / \mathrm{sec}$ & $2.3 \%$ \\
\hline
\end{tabular}




\subsection{Two-Model Hybrid Calibration Methodology}

In the two previous sections, parametric and non-parametric calibration methodologies were used to characterize a compliant, 6-axis, force/torque sensor as both approaches gave satisfactory results. The dynamic calibration proposed in the previous section had the ability to effectively characterize the behaviour of the compliant sensor and was able to significantly reduce the hysteresis effects. However, the value of good fit of the dynamic model is $93.30 \%$ when a state space estimation model is used, which can be improved by incorporating a non-parametric machine learning based calibration. Using Gaussian process regression (GPR) models alone gave excellent results and high-quality

performance with a value of good fi of up to $99 \%$ as reported in Table I, but the speed of estimation was around 12,000 obs/sec which can be increased to enhance the sensing performance as required for real-time applications where high speeds of prediction is necessary. Results achieved from different GPR model can be seen in Figure 7.10

The proposed two-model based hybrid calibration method aims to better improve the sensing quality of the compliant sensor by taking the advantages of the dynamic modelling of the state space model and the excellent performance of the Gaussian process regression models. This can be achieved by combining a state space estimation model with a Gaussian process regression to create a single calibration system that has the advantages of both models at the same time.

The state space model is used to estimate the linear force data, which is achieved using data-driven modelling and an identification process. The input of the system is the output voltage of the compliant force/torque sensor and the output is the actual force data. Once the estimation model is obtained, the difference of the actual force 
Table 7.3: A comparison between the proposed novel dynamic calibration methodology of the state space Gaussian process regression (SSGPR) and the GPR model alone in terms of the RMSE, the speed of prediction and the fitness value .

\begin{tabular}{cccc}
\hline Model & $R^{2}$ & Estimation Speed & RMSE \\
\hline SSGPR & $100 \%$ & 45,000 obs $/$ sec & $1.46 \%$ \\
\hline GPR alone & $100 \%$ & 10,000 obs $/$ sec & $1.86 \%$ \\
\hline \hline
\end{tabular}

data and the estimated data (from the compliant sensor) gets relayed as the output target for the Gaussian process regression model. Its input is the voltage of the compliant sensor. The Gaussian process regression model uses these inputs and outputs to estimate the difference (ie. error) of the compliant sensor and to learn the pattern of the system. Once it has the data, the model is then trained to achieve a useful error estimator. Once both models are obtained, the proposed novel two-model-based dynamic calibration is the sum of the output of the main state space dynamic model and the output of the error estimator.

The results obtained from this calibration method (the SSGPR method) are more accurate than each of the previous models as the RMSE was reduced from $1.86 \%$ to $1.46 \%$, more importantly the estimation speed was significantly increased from 10,000 $\mathrm{obs} / \mathrm{sec}$ to $45,000 \mathrm{obs} / \mathrm{sec}$. Table II demonstrates a comparison between the SSGPR model and GPR without incorporating the state space dynamic model. Table 7.3 summarizes a comparison between the proposed estimation models.

\subsection{Conclusion}

The different effective calibration methodologies for compliant force/torque sensors were proposed and applied. This incorporates parametric calibration methods and 
non-parametric machine learning-based calibration strategies. For the parametric calibration, linear dynamic models were obtained through a system identification process and data-driven dynamic modelling which includes transfer function and state space models. These estimation models were able to accurately estimate the applied force and torque. More importantly, they were able to efficiently compensate for the hysteresis effects as it was significantly reduced by $95 \%$ compared to the conventional calibration methodologies.

For the small deflections generated by a relatively small amount of force between zero to $25 \mathrm{~N}$, the conventional calibration gave very accurate results with RMSE of $6.2 \times 10^{-8} \mathrm{~N}$ and R-squared of $100 \%$. However, this method could generate errors in the case where large deflections and high force ranging $1000 \mathrm{~N}$ is applied, since the non-linearity increases in this situation.

To address this issue, we proposed a novel, two-model-based dynamic calibration procedure that combines a dynamic estimation model based on state space representation and a machine learning model based on Gaussian process regression (GPR) which was capable of improving the performance the compliant sensor at a high amount of force application. This novel calibration method was also able to significantly increase the speed of prediction compared to the conventional calibration as well as these models when used on their own. 


\section{Chapter 8}

\section{Conclusions and Recommendations for}

\section{Future Work}

Compliant force and torque sensors have shown their significance in several applications such as biomechanics, rehabilitation systems, human-machine interface, ground reaction force and gait measurements. BalanceAid project for instance, is a comprehensive human control and balance system being developed and improved in the ABL laboratory at Carleton University. The work explained in the current thesis is a part of this project that aims to develop an ambulatory balance assessment device and a feedback system to enhance the mobility and balance of the impaired gait person. Force and torque sensor data is a vital information for the BalanceAid system to provide a feedback when the patient is at risk of falling. Three force components including normal and shear forces as well as the three moment components must be measured to be able to obtain the full ground reaction force which is required for fall prevention and maintain balance. 
This thesis demonstrated the full development process of five different prototypes of compliant, lightweight, small size, multi-dimensional force and torque sensors that were able to measure the complete ground reaction force and moment. The technologies involved in these sensors are based on fiber-optic sensing principles including light intensity modulation and fiber Bragg grating techniques that relies on light wavelength variation. Also, a wearable, compliant 6-axis force and torque sensor that combines two sensing techniques in one sensor system: the optical light intensity modulation and FlexiForce pressure sensors based on force sensitive resistor. These developed compliant sensors exhibited a complex nonlinear dynamic behaviour due to the viscoelastic properties of the flexible material used in the sensors. This behaviour cannot be fully described using the conventional calibration methods, thus, several novel calibration and sensor characterization methodologies were proposed and used which have confirmed their effectiveness in addressing this dynamic behaviour. The calibration methods were also able to characterize the hysteresis, reduce the errors, increase the speed of estimation and improve the dynamic response of the proposed compliant force and torque sensors. The conclusions of this thesis are presented as follows:

\subsection{Conclusions}

\subsubsection{Design and Implementation of A Compliant 3-Axis Fiber-Optic Based Force Sensor}

A novel, high-precision, compliant, triaxial fiber-optic force sensor operates based on light intensity modulation was designed, manufactured and calibrated successfully. The 
sensor was able to measure the magnitude and the direction of the applied normal and shear forces simultaneously with a potential to adjust the sensing range. Both static and dynamic tests were performed to evaluate the sensor's characteristic response in different loading conditions. A dynamic linear and nonlinear system identification models have been used and both have shown satisfactory results. A Hammerstein Wiener estimation model was necessary to characterize both the linearity and nonlinearity of the sensor since it incorporated an integrated linear and nonlinear models. The model has shown a high-quality performance with a fitness value of $F x=94.12 \%$, $F y=92.25 \%$ and $F z=93.20 \%$. The crosstalk errors were also significantly reduced in this prototype through its novel mechanical design and sensing mechanism which were between $0.37 \%$ and $4.01 \%$, before using any mathematical or any other crosstalk compensation

\subsubsection{Development of 6-DoF Sensor for Total Ground Reaction Force Based on Two Sensing Technologies}

A novel ambulatory force and moment sensor system for the complete ground reaction measurement was designed and prototyped. The sensor was developed to replace the previous traditional pressure insole used in the BalanceAid project that was only able to measure one component of ground reaction force (normal force). The sensor is compliant, small size and has minimal disturbance to the natural gait pattern. Unlike the conventional multi-axis force transducers this sensor does not cause fatigue or any discomfort to the user due to its high flexibility and its lightweight. The sensing system combines two different sensing technologies, the fiber-optic based on light intensity modulation the and flexible pressure matrix (FlexiForce sensors) to measure the normal 
and shear forces and to capture the applied moments. The sensor was validated through an actual gait measurement scenario by mounting two sensing units underneath a foot to detect the full ground reaction force and moment during gait. The sensor was successfully calibrated using linear and nonlinear calibration methods based on neural network with RMSE of $9.39 \%$ and $5.21 \%$ for the linear and nonlinear calibration respectively. Stephanie Eng was involved in this work.

\subsubsection{Design, Development and Calibration of a Lightweight, Compliant Six-Axis Optical Force/Torque Sensor}

A multi-dimensional force and torque sensor was designed, developed and calibrated. The sensor is based on fiber-optic technology with a six degree of freedom sensing capability. The sensor incorporates only six fiber-optic based sensing element that were able to accurately measure the six relevant force and torque components. The sensor is compliant and light weight with no integrated conditioners or amplifications. The simplicity in its configuration and design have led to simpler sensor fabrication and straightforward assembly process, resulting in a low price 6-axis $\mathrm{F} / \mathrm{T}$ sensor. The sensor has the advantage of the ability to increase or decrease the measuring range and sensor size based on the intended biomechanical application.

A novel calibration methodology named Least Squares Decision Tree (LSDT) was also developed and applied to obtain a high precision measurement of forces and torques. The RMSE of LSDT for each axis were as follows $F z=0.17 \%, F x=0.72 \%$, $F y=0.56 \%, T x=0.16 \%, T y=0.75 \%, T z=0.84 \%$. This confirms the high-quality performance of this sensor when the novel LSDT calibration is used. 


\subsubsection{Development of FBG-Based Normal and Shear Force Sensing Elements}

a compliant, small size, lightweight, fiber Bragg gratings-based, normal and shear force sensing elements have been designed, fabricated and characterized successfully. The sensors were evaluated through several experiments using static and dynamic loading tests. Both normal and shear force sensing elements have shown a strong ability to accurately measure the applied force with a possibility to adjust the force sensing range based on the desired intended application. Due to its compliance, non-electrical conductivity, immunity to electromagnetic interference and its wide force measurement range, the sensing elements exhibit excellent biocompatibility that can be used in several biomechanical measurements such as in human-machine interface, total ground reaction force (GRF), and any other applications where flexibility of the sensor is desirable, and rigidity is an issue. a novel effective calibration methodology based on the nonlinear autoregressive exogenous (NARX) was applied, obtaining accurate results and high-quality performance.

\subsubsection{Novel Calibration Methodologies for Compliant Multi- Axis Force/Torque Sensors}

Novel parametric calibration methods and non-paramedic machine learning-based calibration strategies were proposed and applied in this thesis. Preliminary results investigating the functionality of these calibration methodologies indicate their strong abilities to characterize the complex nonlinear dynamic behaviours of compliant force and torque sensors. Dynamic models were obtained through a system identification 
process and data-driven dynamic modelling including state space and transfer function models which were able to significantly reduce the hysteresis phenomenon by $95 \%$ compared to the conventional calibration methodologies. However, the sensor behaviour was not fully capture since these models are linear, thus; a novel two-model based dynamic calibration procedure was proposed. This approach that combines a dynamic estimation model based on state space representation and a static machine learning model based on Gaussian process regression (GPR) which was capable of improving the performance the compliant sensor even when a high amount of force is applied. This novel calibration method was also able to significantly increase the speed of prediction compared to the conventional calibration.

\subsection{Future Work}

As a first version of each sensor prototype the experimental results obtained from various loading conditions and testing scenarios have confirmed the high-quality performance of force and torque sensing. However, there are improvements that can be performed for a next version of each device.

\subsubsection{Sensor Modifications and Improvements}

The current version of each sensor is compliant and lightweight and is functional to accurately measure the three force components: $F x, F y$ and $F z$ as well as the three components of the applied torque: Tx, Ty and Tz. However, more miniaturization could be implemented to be even more comfortable and user compatible particularly when is the intended application is ground reaction force measurement 
and rehabilitation systems where the sensor is attached to a shoe or to a personalized assistive device.

The current thickness of the current sensors is $12 \mathrm{~mm}$ and $5 \mathrm{~cm}$ square as they were made to be large for ease of production, but could be reduced, improving portability and having less disturbance of natural gait pattern.

The proposed sensors have a high measuring range up to $1000 \mathrm{~N}$, but in case of overweight users, the sudden step can be higher than their normal weight, thus; the sensors should also be able to withstand higher normal forces. This can be achieved by adjusting the flexibility of the rubber used through its mixing ratio and therefore increase the measuring range. However, the rigidity of the sensor top and bottom layers should also be increased to avoid any deformation that might occur to the layers which can be any material including metals such as steel, aluminum, or carbon fiber, depending on the application restrictions. These definitely provide options that can be decided for each application separately. Metallic layers can add extra strength and support the PLA layer as well. In the current versions the four rubber blocks sandwiched between these layers have high viscoelastic properties compared to PLA, making the whole deflection occurs within the rubber.

\subsubsection{Development of FBG-Based, 6-Dof insole Sensor for Total Ground Reaction Force and Moment}

To measure the six components of the applied force and torque, at least six force signals from six force sensing elements are required [224]. In this research, the necessary types of force sensing elements required to successfully achieve any multi-axis force and torque sensor were developed and prototyped which include the developed FBG-based normal 
and shear force sensors reported in chapter 6 . This can be obtained by incorporating three shear force sensors to measure Fx, Fy and Tz; and three normal force sensing elements to measure Fz, Tx and Ty.

In the future work we aim to develop a 6-Dof thin sensor sheet that can be easily integrated in any shoe and can accurately measure the plantar distributive force and moments as well as the temperature beneath a foot using only one optical fiber with FBG-based sensing technique.

\subsubsection{BalanceAid Biofeedback Integration}

Various biofeedback systems in literature have been developed to enhance balance performance based on several technologies, such as haptic biofeedback [296-298], visual biofeedback [299-302] and auditory biofeedback [303-305] systems. Such methods are being used to compensate for the reduced sensory information resulting from trauma or aging.

During a walk or standing state, real-time tracking of center of pressure (COP) and center of mass (COM) of the human body is conducive to an enhanced balance performance ??. A floor-fixed force plate [306, 307] and motion capture system [308, 309] with infrared cameras and reflective markers attached to the body are used to detect $\mathrm{COP}$ and COM. However, these standard measurement systems are only available in a laboratory environment, which can significantly limit the assessment of gait measurement performance. The future work is to develop a biofeedback system for balance control using the developed ambulatory sensing system for the complete ground reaction force and moment. The COP is closely related to the displacement of COM, which has been extensively used for postural balance assessments in several 
studies [310]. Therefore, the proposed sensors will be used to predict the positions of the $\mathrm{COP}$ and $\mathrm{COM}$ from the measured force and moment signals. By obtaining this information, a biofeedback system based on vibrotactile actuators can be implemented to give a reminding signal by way of vibrations to the wearer for postural adjustment in order to prevent falls that are a result of poor balance. Furthermore, collaborative experiments should be conducted to evaluate the effect of the overall system on a user; this could include testing various biofeedback algorithms together with these sensors as well as human factor aspects of wearing the sensors.

\subsubsection{Assistive Device Feedback Integration}

Assistive strategies such as wearable lower-limb exoskeletons represent one of the most effective solutions for locomotion assistance and rehabilitation applications. Lower-limb assistive devices are used to provide extra energy during daily bases locomotive activities for the elderly and for people surfing from muscle weakness and disabilities. The single-joint exoskeleton is very practical and requires less complicated control algorithms than the multi-jointed exoskeleton. Lower-limb rehabilitation devices with single-joint assistance are classified under three types: hip [286, 311-314], knee [315-319] and ankle exoskeletons [320-323]. In the future work we aim to develop a one degree-of-freedom knee assisting joint as a simplified exoskeleton system for sit-to-stand and stand-to-sit tasks as well as to supply extra torque to help regain normal gait pattern, adjust one's posture and maintain balance.

A closed-loop feedback system using the proposed sensors instrumented into the shoes will be developed. The total ground reaction force and moment information measured by these sensors will be used to estimate the centre of pressure (COP) and 
centre of mass $(\mathrm{COM})$. As the positions of the $\mathrm{COP}$ and $\mathrm{COM}$ exceed a predefined range, an instantaneous feedback signal will be delivered to the controller which in turn gives the appropriate command to the motors of the knee exoskeleton in order to help the wearer recover the defined position of the COP/COM. 


\section{References}

[1] O. Al-Mai, M. Ahmadi, and J. Albert, "A compliant 3-axis fiber-optic force sensor for biomechanical measurement," IEEE Sensors Journal, vol. 17, no. 20, pp. 6549-6557, 2017.

[2] — , "Design, development and calibration of a lightweight, compliant six-axis optical force/torque sensor," IEEE Sensors Journal, vol. 18, no. 17, pp. 7005-7014, 2018.

[3] S. Eng, O. Al-Mai, and M. Ahmadi, "A 6 dof, wearable, compliant shoe sensor for total ground reaction measurement," IEEE Transactions on Instrumentation and Measurement, no. 99, pp. 1-9, 2018.

[4] O. Al-Mai, M. Ahmadi, and J. Albert, "Fiber-optic based, force and torque compliant sensing calibration strategies," International Conference on Control, Dynamic Systems, and Robotics (CDSR), 2019, no. 144, p. 1, 2019.

[5] M. d. F. F. Domingues and A. Radwan, Optical Fiber Sensors for loT and Smart Devices. Springer Publishing Company, Incorporated, 2017.

[6] P. Roriz, O. Frazão, A. B. Lobo-Ribeiro, J. L. Santos, and J. A. Simões, "Review of fiber-optic pressure sensors for biomedical and biomechanical applications," Journal of biomedical optics, vol. 18, no. 5, pp. 050 903-050 903, 2013. 
[7] W.-C. Wang, W. R. Ledoux, B. J. Sangeorzan, and P. G. Reinhall, "A shear and plantar pressure sensor based on fiber-optic bend loss," Journal of rehabilitation research and development, vol. 42, no. 3, p. 315, 2005.

[8] C.-Y. Huang, W.-J. Wu, S.-C. Fu, P. G. Reinhall, and W.-C. Wang, "A wireless composite optical bend loss sensor for pressure and shear measurement," in Health Monitoring and Smart Nondestructive Evaluation of Structural and Biological Systems V, vol. 6177. International Society for Optics and Photonics, 2006, p. 61770W.

[9] A. G. Leal-Junior, A. Frizera, L. M. Avellar, C. Marques, and M. J. Pontes, "Polymer optical fiber for in-shoe monitoring of ground reaction forces during the gait," IEEE Sensors Journal, vol. 18, no. 6, pp. 2362-2368, 2018.

[10] P. Polygerinos, L. D. Seneviratne, R. Razavi, T. Schaeffter, and K. Althoefer, "Triaxial catheter-tip force sensor for mri-guided cardiac procedures," IEEE/ASME Transactions on mechatronics, vol. 18, no. 1, pp. 386-396, 2012.

[11] M. C. Yip, S. G. Yuen, and R. D. Howe, "A robust uniaxial force sensor for minimally invasive surgery," IEEE transactions on biomedical engineering, vol. 57, no. 5, pp. 1008-1011, 2010.

[12] T. L. Bützer, M. D. Rinderknecht, G. H. Johannes, W. L. Popp, R. Lehner, O. Lambercy, and R. Gassert, "Design and evaluation of a fiber-optic grip force sensor with compliant 3d-printable structure for (f) mri applications," Journal of Sensors, vol. 2016, 2016.

[13] J. Arata, S. Terakawa, J. Sulzer, R. Gassert, and H. Fujimoto, "Mri-compatible grasping force sensor with an inclined double parallel structure using fiber optics," 
Transactions of the Institute of Systems, Control and Information Engineers, vol. 26, no. 3, pp. 110-116, 2013.

[14] S. Crea, M. Donati, S. M. M. De Rossi, C. M. Oddo, and N. Vitiello, "A wireless flexible sensorized insole for gait analysis," Sensors, vol. 14, no. 1, pp. 1073-1093, 2014.

[15] S. S. Yin and P. Ruffin, Fiber optic sensors. Wiley Online Library, 2002.

[16] Y.-F. Zhang, C.-Y. Hong, R. Ahmed, and Z. Ahmed, "A fiber Bragg grating based sensing platform fabricated by fused deposition modeling process for plantar pressure measurement," Measurement, vol. 112, pp. 74-79, 2017.

[17] T.-C. Liang, J.-J. Lin, and L.-Y. Guo, "Plantar pressure detection with fiber Bragg gratings sensing system," Sensors, vol. 16, no. 10, p. 1766, 2016.

[18] A. V. Koulaxouzidis, V. C. Roberts, M. J. Holmes, and V. A. Handerek, "Development of a fiber Bragg grating sensor for in-shoe shear stress measurement: design and preliminary results," in Applications of Optical Fiber Sensors, vol. 4074. International Society for Optics and Photonics, 2000, pp. $79-89$.

[19] R. Suresh and S. C. Tjin, "Effects of dimensional and material parameters and cross-coupling on FBG based shear force sensor," Sensors and Actuators A: Physical, vol. 120, no. 1, pp. 26-36, 2005.

[20] R. Suresh, S. C. Tjin, and S. Bhalla, "Multi-component force measurement using embedded fiber Bragg grating," Optics \& Laser Technology, vol. 41, no. 4, pp. 431-440, 2009.

[21] Z. F. Zhang, X. M. Tao, H. P. Zhang, and B. Zhu, "Soft fiber optic sensors 
for precision measurement of shear stress and pressure," IEEE Sensors Journal, vol. 13, no. 5, pp. 1478-1482, 2013.

[22] M. F. Domingues, C. Tavares, C. Leitão, A. Frizera-Neto, N. Alberto, C. Marques, A. Radwan, J. Rodriguez, O. Postolache, E. Rocon et al., "Insole optical fiber Bragg grating sensors network for dynamic vertical force monitoring," Journal of Biomedical Optics, vol. 22, no. 9, pp. 091507-091507, 2017.

[23] G. Voskerician, M. S. Shive, R. S. Shawgo, H. Von Recum, J. M. Anderson, M. J. Cima, and R. Langer, "Biocompatibility and biofouling of mems drug delivery devices," Biomaterials, vol. 24, no. 11, pp. 1959-1967, 2003.

[24] A. G. Mignani and F. Baldini, "Biomedical sensors using optical fibres," Reports on Progress in Physics, vol. 59, no. 1, p. 1, 1996.

[25] J.-J. Park and G.-S. Kim, "Development of the 6-axis force/moment sensor for an intelligent robot's gripper," Sensors and Actuators A: Physical, vol. 118, no. 1, pp. 127-134, 2005.

[26] T. Dwarakanath and G. Bhutani, "Beam type hexapod structure based six component force-torque sensor," Mechatronics, vol. 21, no. 8, pp. 1279-1287, 2011.

[27] L. Schickl, K. Dorer, M. Wülker, and U. Hochberg, "Development of a six-axis force and torque sensor for the humanoid robot sweaty 2.0," in the 11th Workshop on Humanoid Soccer Robots at 16th IEEE-RAS International Conference on Humanoid Robots, Cancun, 2016.

[28] S. Hu, H. Wang, Y. Wang, and Z. Liu, "Design of a novel six-axis wrist force 
sensor," Sensors, vol. 18, no. 9, p. 3120, 2018.

[29] M. E. Ladd and H. H. Quick, "Reduction of resonant rf heating in intravascular catheters using coaxial chokes," Magnetic resonance in medicine, vol. 43, no. 4, pp. 615-619, 2000.

[30] W. Jin, "Multiplexed FBG sensors and their applications," in International Symposium on Photonics and Applications. International Society for Optics and Photonics, 1999, pp. 468-479.

[31] C.-S. Kim, T. H. Lee, Y. S. Yu, Y.-G. Han, S. B. Lee, and M. Y. Jeong, "Multi-point interrogation of FBG sensors using cascaded flexible wavelength-division sagnac loop filters," Optics express, vol. 14, no. 19, pp. 8546-8551, 2006.

[32] M. Davis and A. Kersey, "Matched-filter interrogation technique for fibre Bragg grating arrays," Electronics letters, vol. 31, no. 10, pp. 822-823, 1995.

[33] D. A. Jackson, L. Reekie, J. Archambault, and A. L. Ribeiro, "Simple multiplexing scheme for a fiber-optic grating sensor network," Optics Letters, vol. 18, no. 14, pp. 1192-1194, 1993.

[34] D. J. Cooper, T. Coroy, and P. W. Smith, "Time-division multiplexing of large serial fiber-optic Bragg grating sensor arrays," Applied Optics, vol. 40, no. 16, pp. 2643-2654, 2001.

[35] B. Dong, S. He, Y. Hushu, W. Tianda, F. Lvjun, T. Guo, and Q. Zhao, "Combined time-and wavelength-division-multiplexing demodulation technique of fiber grating sensor arrays using a tunable pulsed laser," Applied optics, vol. 46, no. 7, pp. 1015-1018, 2007. 
[36] S. C. Tjin, J. Hao, Y.-Z. Lam, Y. C. Ho, and B. K. Ng, "A pressure sensor using fiber Bragg grating," Fiber \& Integrated Optics, vol. 20, no. 1, pp. 59-69, 2001.

[37] V. Mishra, N. Singh, U. Tiwari, and P. Kapur, "Fiber grating sensors in medicine: Current and emerging applications," Sensors and Actuators A: Physical, vol. 167, no. 2, pp. 279-290, 2011.

[38] A. Ungar, M. Rafanelli, I. Iacomelli, M. A. Brunetti, A. Ceccofiglio, F. Tesi, and N. Marchionni, "Fall prevention in the elderly," Clinical Cases in mineral and bone metabolism, vol. 10, no. 2, p. 91, 2013.

[39] M. O. Gu, M. Y. Jeon, H. J. Kim, and Y. Eun, "A review of exercise interventions for fall prevention in the elderly," Journal of Korean Academy of Nursing, vol. 35, no. 6, pp. 1101-1112, 2005.

[40] K. Milisen, N. Staelens, R. Schwendimann, L. De Paepe, J. Verhaeghe, T. Braes, S. Boonen, W. Pelemans, R. W. Kressig, and E. Dejaeger, "Fall prediction in inpatients by bedside nurses using the st. thomas's risk assessment tool in falling elderly inpatients (stratify) instrument: a multicenter study," Journal of the American Geriatrics Society, vol. 55, no. 5, pp. 725-733, 2007.

[41] J. Swanenburg, E. D. de Bruin, D. Uebelhart, and T. Mulder, "Falls prediction in elderly people: a 1-year prospective study," Gait \& posture, vol. 31, no. 3, pp. 317-321, 2010.

[42] R. Cham and M. S. Redfern, "Changes in gait when anticipating slippery floors," Gait $\mathscr{G}$ posture, vol. 15, no. 2, pp. 159-171, 2002.

[43] L. A. Lavery, K. R. Higgins, J. La Fontaine, R. G. Zamorano, G. P. Constantinides, and P. J. Kim, "Randomised clinical trial to compare total 
contact casts, healing sandals and a shear-reducing removable boot to heal diabetic foot ulcers," International wound journal, vol. 12, no. 6, pp. 710-715, 2015.

[44] M. Yavuz, A. Ersen, J. Hartos, B. Schwarz, A. G. Garrett, L. A. Lavery, D. K. Wukich, and L. S. Adams, "Plantar shear stress in individuals with a history of diabetic foot ulcer: an emerging predictive marker for foot ulceration," Diabetes care, vol. 40, no. 2, pp. e14-e15, 2017.

[45] D. G. Armstrong, A. J. Boulton, and S. A. Bus, "Diabetic foot ulcers and their recurrence," New England Journal of Medicine, vol. 376, no. 24, pp. 2367-2375, 2017.

[46] M. E. Fernando, R. G. Crowther, P. A. Lazzarini, K. S. Sangla, P. Buttner, and J. Golledge, "Gait parameters of people with diabetes-related neuropathic plantar foot ulcers," Clinical Biomechanics, vol. 37, pp. 98-107, 2016.

[47] M. Yavuz, "American society of biomechanics clinical biomechanics award 2012: plantar shear stress distributions in diabetic patients with and without neuropathy," Clinical biomechanics, vol. 29, no. 2, pp. 223-229, 2014.

[48] P. G. Zarzar, "Force sensing insole for a balance enhancement system," Ph.D. dissertation, Carleton University Ottawa, 2014.

[49] M. Vukobratović, H. M. Herr, B. Borovac, M. Raković, M. Popovic, A. Hofmann, M. JOVANOVIĆ, and V. Potkonjak, "Biological principles of control selection for a humanoid robot's dynamic balance preservation," International Journal of Humanoid Robotics, vol. 5, no. 04, pp. 639-678, 2008.

[50] L. E. C. Lizama, M. Pijnappels, N. P. Reeves, S. M. Verschueren, and J. H. van 
Dieën, "Centre of pressure or centre of mass feedback in mediolateral balance assessment," Journal of biomechanics, vol. 48, no. 3, pp. 539-543, 2015.

[51] K. Grattan and T. Sun, "Fiber optic sensor technology: an overview," Sensors and Actuators A: Physical, vol. 82, no. 1, pp. 40-61, 2000.

[52] P. M. Tracey, "Intrinsic fiber-optic sensors," Industry Applications, IEEE Transactions on, vol. 27, no. 1, pp. 96-98, 1991.

[53] S.-K. Yao and C. K. Asawa, "Fiber optical intensity sensors," Selected Areas in Communications, IEEE Journal on, vol. 1, no. 3, pp. 562-575, 1983.

[54] C. Batchellor and C. Edge, "Some recent advances in fibre-optic sensors," Electronics $\& 3$ communication engineering journal, vol. 2, no. 5, pp. 175-184, 1990.

[55] A. Marzuki, H. Novianto, W. Hidayat, and A. Setyawan, "Intensity modulated fiber sensor configuration equipped with a variable fiber optic attenuator," in The Second International Conference on Technological Advances in Electrical, Electronics and Computer Engineering (TAEECE2014). The Society of Digital Information and Wireless Communication, 2014, pp. 205-208.

[56] N. Rana, "Application of force sensing resistor ( $\mathrm{fsr}$ ) in design of pressure scanning system for plantar pressure measurement," in Computer and Electrical Engineering, 2009. ICCEE'09. Second International Conference on, vol. 2. IEEE, 2009, pp. 678-685.

[57] F. Martínez-Martí, M. S. Martínez-García, S. G. García-Díaz, J. García-Jiménez, A. J. Palma, and M. A. Carvajal, "Embedded sensor insole for wireless 
measurement of gait parameters," Australasian Physical \& Engineering Sciences in Medicine, vol. 37, no. 1, pp. 25-35, 2014.

[58] M. Amjadi, M. S. Kim, and I. Park, "Flexible and sensitive foot pad for sole distributed force detection," in Nanotechnology (IEEE-NANO), 2014 IEEE 14th International Conference on. IEEE, 2014, pp. 764-767.

[59] W. Chen, Y. Xu, J. Wang, and J. Zhang, "Kinematic analysis of human gait based on wearable sensor system for gait rehabilitation," Journal of Medical and Biological Engineering, vol. 36, no. 6, pp. 843-856, 2016.

[60] L. Motha, J. Kim, and W. S. Kim, "Instrumented rubber insole for plantar pressure sensing," Organic Electronics, vol. 23, pp. 82-86, 2015.

[61] K. F. Lei, K.-F. Lee, and M.-Y. Lee, "Development of a flexible pdms capacitive pressure sensor for plantar pressure measurement," Microelectronic Engineering, vol. 99, pp. 1-5, 2012.

[62] B. J. Mortimer, G. A. Zets, B. J. Altenbernd, and T. Goonetilleke, "Development of a planar shear sensor," in Engineering in Medicine and Biology Society (EMBC), 2016 IEEE 38th Annual International Conference of the. IEEE, 2016, pp. $2030-2033$.

[63] X. Zhang, Y. Zhao, Z. Duan, and Y. Liu, "Design and test of a soft plantar force measurement system for gait detection," Sensors, vol. 12, no. 12, pp. $16628-16640,2012$.

[64] C. S. Morère, E. Surażyński, A. R. Pérez-Tabernero, E. Vihriälä, and T. Myllylä, "Mems technology sensors as a more advantageous technique for measuring foot plantar pressure and balance in humans," Journal of Sensors, vol. 2016, 2016. 
[65] S. Rajala, S. Tuukkanen, and J. Halttunen, "Characteristics of piezoelectric polymer film sensors with solution-processable graphene-based electrode materials," IEEE Sensors Journal, vol. 15, no. 6, pp. 3102-3109, 2015.

[66] S. Rajala, T. Salpavaara, and S. Tuukkanen, "Testing and comparing of film-type sensor materials in measurement of plantar pressure distribution," in Engineering in Medicine and Biology Society (EMBC), 2016 IEEE 38th Annual International Conference of the. IEEE, 2016, pp. 251-254.

[67] S. Rajala, M. Vuoriluoto, O. J. Rojas, S. Franssila, and S. Tuukkanen, "Piezoelectric sensitivity measurements of cellulose nanofibril sensors," Proc. XXI IMEKO World Congr., Prague, Czech Republic, 2015.

[68] J. W. Sohn, J. Jeon, and S.-B. Choi, "An investigation on dynamic signals of mfc and pvdf sensors: Experimental work," Advances in Mechanical Engineering, vol. 5, p. 420345, 2013.

[69] E. Klimiec, K. Zaraska, J. Piekarski, P. Guzdek, G. Kołaszczyński, and B. Jasiewicz, "Durable sensors for measurement of foot plantar pressure with piezoelectric polyvinylidene fluoride foil," Sensors and Actuators A: Physical, vol. 247, pp. 504-513, 2016.

[70] P. Cosseddu, F. Viola, S. Lai, L. Raffo, and A. Bonfiglio, "A temperature transducer based on a low-voltage organic thin-film transistor detecting pyroelectric effect," IEEE Electron Device Letters, vol. 35, no. 12, pp. 1296-1298, 2014.

[71] S. R. Anton and H. A. Sodano, "A review of power harvesting using piezoelectric materials (2003-2006)," Smart materials and Structures, vol. 16, no. 3, p. R1, 
2007.

[72] T. Patra, "A simple fiber optic displacement sensor for measurement of light intensity with the displacement," International Journal of Latest Trends in Engineering and Technology (IJLTET)l, vol. 2, pp. 161-165, 2013.

[73] D. A. Krohn, "Intensity modulated fiber optic sensors overview," in Cambridge Symposium-Fiber/LASE'86. International Society for Optics and Photonics, 1987, pp. 2-11.

[74] P. Roriz, A. Ramos, J. L. Santos, and J. A. Simões, "Fiber optic intensity-modulated sensors: A review in biomechanics," Photonic Sensors, vol. 2, no. 4, pp. 315-330, 2012.

[75] S. Silvestri and E. Schena, Optical-fiber measurement systems for medical applications. Citeseer, 2011.

[76] D. J. Margolis, J. Knauss, W. Bilker, and M. Baumgarten, "Medical conditions as risk factors for pressure ulcers in an outpatient setting," Age and ageing, vol. 32, no. 3, pp. 259-264, 2003.

[77] M. M. Rodgers, "Dynamic biomechanics of the normal foot and ankle during walking and running," Physical Therapy, vol. 68, no. 12, pp. 1822-1830, 1988. [Online]. Available: http://ptjournal.apta.org/content/68/12/1822

[78] E. S. Sazonov, G. Fulk, J. Hill, Y. Schütz, and R. Browning, "Monitoring of posture allocations and activities by a shoe-based wearable sensor," Biomedical Engineering, IEEE Transactions on, vol. 58, no. 4, pp. 983-990, 2011.

[79] P. Bonato et al., "Wearable sensors/systems and their impact on biomedical 
engineering," IEEE Engineering in Medicine and Biology Magazine, vol. 22, no. 3, pp. 18-20, 2003.

[80] Y. Feng, Y. Ge, and Q. Song, "A human identification method based on dynamic plantar pressure distribution," in Information and Automation (ICIA), 2011 IEEE International Conference on. IEEE, 2011, pp. 329-332.

[81] F. Neaga, D. Moga, D. Petreus, M. Munteanu, and N. Stroia, "A wireless system for monitoring the progressive loading of lower limb in post-traumatic rehabilitation," in International Conference on Advancements of Medicine and Health Care through Technology. Springer, 2011, pp. 54-59.

[82] C. Wada, Y. Sugimura, T. Ienaga, Y. Kimuro, F. Wada, K. Hachisuka, and T. Tsuji, "Development of a rehabilitation support system with a shoe-type measurement device for walking," in SICE Annual Conference 2010, Proceedings of. IEEE, 2010, pp. 2534-2537.

[83] A. Gefen, "Pressure-sensing devices for assessment of soft tissue loading under bony prominences: technological concepts and clinical utilization." Wounds: a compendium of clinical research and practice, vol. 19, no. 12, pp. 350-362, 2007.

[84] K. Kim, B. Floyd, J. Mehta, H. Yoon, C.-M. Hung, D. Bravo, T. Dickson, X. Guo, R. Li, N. Trichy et al., "The feasibility of on-chip interconnection using antennas," in Proceedings of the 2005 IEEE/ACM International conference on Computer-aided design. IEEE Computer Society, 2005, pp. 979-984.

[85] V. Karam, P. H. Popplewell, A. Shamim, J. Rogers, and C. Plett, “A 6.3 ghz bfsk transmitter with on-chip antenna for self-powered medical sensor applications," in 2007 IEEE Radio Frequency Integrated Circuits (RFIC) Symposium. IEEE, 
2007, pp. 101-104.

[86] W.-C. Wang, J. Dee, W. Ledoux, B. Sangeorzan, and P. Reinhall, "Development of a directional sensitive pressure and shear sensor," in Proc. SPIE, vol. 4702, 2002, pp. 212-220.

[87] Y. Otsuka, Y. Koyama, and K. Watanabe, "Monitoring of plantar pressure in gait based on hetero-core optical fiber sensor," Procedia Engineering, vol. 87, pp. 1465-1468, 2014.

[88] C.-Y. Huang, W.-C. Wang, W.-J. Wu, and W. R. Ledoux, "Composite optical bend loss sensor for pressure and shear measurement," IEEE Sensors Journal, vol. 7, no. 11, pp. 1554-1565, 2007.

[89] E. Pinet and C. Hamel, "True challenges of disposable optical fiber sensors for clinical environment," in Third European Workshop on Optical Fibre Sensors, vol. 6619. International Society for Optics and Photonics, 2007, p. 66191Q.

[90] R. Munoz, L. Leija, F. Diaz, and J. Alvarez, "3d continuous monitoring system for localization of upper limb based on optical fiber," in Engineering in Medicine and Biology Society, 1995., IEEE 17th Annual Conference, vol. 2. IEEE, 1995, pp. $1595-1596$.

[91] Y. Li, K. Guan, and Z. Hu, "Fiber optic displacement measurement model based on finite reflective surface," Optics \& Laser Technology, vol. 84, pp. 32-39, 2016.

[92] D. H. A. Munap, N. Bidin, S. Islam, M. Abdullah, F. M. Marsin, and M. Yasin, "Fiber optic displacement sensor for industrial applications," IEEE Sensors Journal, vol. 15, no. 9, pp. 4882-4887, 2015.

[93] P. Polygerinos, L. D. Seneviratne, R. Razavi, T. Schaeffter, and K. Althoefer, 
"Triaxial catheter-tip force sensor for mri-guided cardiac procedures," IEEE/ASME Transactions on mechatronics, vol. 18, no. 1, pp. 386-396, 2013.

[94] J. F. Schenck, "The role of magnetic susceptibility in magnetic resonance imaging: Mri magnetic compatibility of the first and second kinds," Medical physics, vol. 23 , no. 6, pp. 815-850, 1996.

[95] S. De Rossi, T. Lenzi, N. Vitiello, M. Donati, A. Persichetti, F. Giovacchini, F. Vecchi, and M. Carrozza, "Development of an in-shoe pressure-sensitive device for gait analysis," in Engineering in Medicine and Biology Society, EMBC, 2011 Annual International Conference of the IEEE. IEEE, 2011, pp. 5637-5640.

[96] H. Jiang, J. Chen, and T. Liu, "Multi-objective design of an FBG sensor network using an improved strength pareto evolutionary algorithm," Sensors and Actuators A: Physical, vol. 220, pp. 230-236, 2014.

[97] Y. B. Lin, K. C. Chang, J. C. Chern, and L. A. Wang, "Packaging methods of fiber-Bragg grating sensors in civil structure applications," IEEE Sensors journal, vol. 5, no. 3, pp. 419-424, 2005.

[98] L. Xiong, D. Zhang, L. Li, and Y. Guo, "Efpi-FBG hybrid sensor for simultaneous measurement of high temperature and large strain," Chinese Optics Letters, vol. 12, no. 12, pp. $120605-120605,2014$.

[99] N. J. van de Berg, J. Dankelman, and J. J. van den Dobbelsteen, "Design of an actively controlled steerable needle with tendon actuation and FBG-based shape sensing," Medical engineering 8 physics, vol. 37, no. 6, pp. 617-622, 2015.

[100] X.-X. Li, W.-X. Ren, and K.-M. Bi, "FBG force-testing ring for bridge cable force 
monitoring and temperature compensation," Sensors and Actuators A: Physical, vol. 223, pp. 105-113, 2015.

[101] T. Li, Y. Tan, Y. Liu, Y. Qu, M. Liu, and Z. Zhou, "A fiber Bragg grating sensing based triaxial vibration sensor," Sensors, vol. 15, no. 9, pp. 24214-24229, 2015.

[102] J.-a. Chen, D. Huang, H.-t. Zhao, Q.-b. Wang, Y. Qiu, and D.-p. Duan, "Fiber Bragg grating-based plane strain monitoring of aerostat envelope structures," Applied optics, vol. 52, no. 19, pp. 4631-4639, 2013.

[103] G. Meltz, W. W. Morey, and W. H. Glenn, "Formation of Bragg gratings in optical fibers by a transverse holographic method," Opt. Lett., vol. 14, no. 15, pp. 823-825, Aug 1989. [Online]. Available: http://ol.osa.org/abstract.cfm?URI=ol-14-15-823

[104] N. Singh, S. C. Jain, A. Aggarwal, and R. Bajpai, "Fibre Bragg grating writing using phase mask technology," 2005.

[105] G. Meltz, W. Morey, and W. Glenn, "Formation of Bragg gratings in optical fibers by a transverse holographic method," Optics letters, vol. 14, no. 15, pp. 823-825, 1989.

[106] J. Cheng, W. Zhu, Z. Huang, and P. Hu, "Experimental and simulation study on thermal gas flowmeter based on fiber Bragg grating coated with silver film," Sensors and Actuators A: Physical, vol. 228, pp. 23-27, 2015.

[107] A. Bertholds and R. Dandliker, "Determination of the individual strain-optic coefficients in single-mode optical fibres," Journal of lightwave technology, vol. 6, no. 1 , pp. 17-20, 1988.

[108] S. James, M. Dockney, and R. Tatam, "Simultaneous independent temperature 
and strain measurement using in-fibre Bragg grating sensors," Electronics Letters, vol. 32, no. 12, pp. 1133-1134, 1996.

[109] Y.-J. Rao, "In-fibre Bragg grating sensors," Measurement science and technology, vol. 8, no. 4, p. 355, 1997.

[110] G. T. Kanellos, G. Papaioannou, D. Tsiokos, C. Mitrogiannis, G. Nianios, and N. Pleros, "Two dimensional polymer-embedded quasi-distributed FBG pressure sensor for biomedical applications," Optics express, vol. 18, no. 1, pp. 179-186, 2010 .

[111] M. R. A. Hassan, M. H. A. Bakar, K. Dambul, and F. R. M. Adikan, "Optical-based sensors for monitoring corrosion of reinforcement rebar via an etched cladding Bragg grating," Sensors, vol. 12, no. 11, pp. 15 820-15826, 2012.

[112] A. G. Prasad, S. Omkar, H. Vikranth, V. Anil, K. Chethana, and S. Asokan, "Design and development of fiber Bragg grating sensing plate for plantar strain measurement and postural stability analysis," Measurement, vol. 47, pp. 789-793, 2014.

[113] M. Nishiyama, H. Sasaki, and K. Watanabe, "Performance characteristics of wearable embedded hetero-core fiber sensors for unconstrained motion analyses," Transactions of the Society of Instrument and Control Engineers, vol. 43, no. 12, pp. 1075-1081, 2007.

[114] G. T. Kanellos, D. Tsiokos, N. Pleros, P. Childs, and S. Pissadakis, "Enhanced durability FBG-based sensor pads for biomedical applications as human-machine interface surfaces," in BioPhotonics, 2011 International Workshop on. IEEE, 2011, pp. $1-3$. 
[115] A. Üneri, M. A. Balicki, J. Handa, P. Gehlbach, R. H. Taylor, and I. Iordachita, "New steady-hand eye robot with micro-force sensing for vitreoretinal surgery," in Biomedical Robotics and Biomechatronics (BioRob), 2010 3rd IEEE RAS and EMBS International Conference on. IEEE, 2010, pp. 814-819.

[116] Y. Jung, M. Jung, K. Lee, and S. Koo, "Ground reaction force estimation using an insole-type pressure mat and joint kinematics during walking," Journal of biomechanics, vol. 47, no. 11, pp. 2693-2699, 2014.

[117] E. S. Sazonov, T. Bumpus, S. Zeigler, and S. Marocco, "Classification of plantar pressure and heel acceleration patterns using neural networks," in Neural Networks, 2005. IJCNN'05. Proceedings. 2005 IEEE International Joint Conference on, vol. 5. IEEE, 2005, pp. 3007-3010.

[118] J. Hao, K. Tan, S. Tjin, C. Liaw, P. R. Chaudhuri, X. Guo, and C. Lu, "Design of a foot-pressure monitoring transducer for diabetic patients based on FBG sensors," in Lasers and Electro-Optics Society, 2003. LEOS 2003. The 16th Annual Meeting of the IEEE, vol. 1. IEEE, 2003, pp. 23-24.

[119] R. Suresh, S. Bhalla, S. C. Tjin, and J. Hao, "Plantar stress monitoring using fiber Bragg grating sensors."

[120] R. Suresh, S. Tjin, and N. Ngo, "Shear force sensing by strain transformation using non-rectilinearly embedded fiber Bragg grating," Sensors and Actuators A: Physical, vol. 116, no. 1, pp. 107-118, 2004.

[121] S. Tjin, R. Suresh, and N. Ngo, "Fiber Bragg grating based shear-force sensor: Modeling and testing," Journal of lightwave technology, vol. 22, no. 7, pp. 1728-1733, 2004. 
[122] M. F. Domingues, C. Tavares, N. Alberto, C. Leitao, P. Antunes, P. Andre, E. R. de Lima, V. Sucasas, A. Radwan, and J. Rodriguez, "Non-invasive insole optical fiber sensor architecture for monitoring foot anomalies," in GLOBECOM 2017-2017 IEEE Global Communications Conference. IEEE, 2017, pp. 1-6.

[123] D. Vilarinho, A. Theodosiou, M. F. Domingues, P. F. da Costa Antunes, K. Kalli, P. S. André, and C. A. Marques, "Foot plantar pressure monitoring with cytop Bragg gratings sensing system." in BIODEVICES, 2018, pp. 25-29.

[124] M. F. Domingues, N. Alberto, C. Leitão, C. Tavares, E. R. de Lima, A. Radwan, V. Sucasas, J. Rodriguez, P. André, and P. Antunes, "Insole optical fiber sensor architecturefor remote gait analysis-an ehealth solution," IEEE Internet of Things Journal, 2017.

[125] E. Weir and L. Culmer, "Fall prevention in the elderly population," Canadian Medical Association Journal, vol. 171, no. 7, pp. 724-724, 2004.

[126] P. Kannus, H. Sievänen, M. Palvanen, T. Järvinen, and J. Parkkari, "Prevention of falls and consequent injuries in elderly people," The Lancet, vol. 366, no. 9500, pp. 1885-1893, 2005.

[127] L.-J. Lin, F.-T. Chiou, and H. H. Cohen, "Slip and fall accident prevention: A review of research, practice, and regulations," Journal of safety research, vol. 26, no. 4, pp. 203-212, 1996.

[128] S. Sadigh, A. Reimers, R. Andersson, and L. Laflamme, "Falls and fall-related injuries among the elderly: a survey of residential-care facilities in a swedish municipality," Journal of community health, vol. 29, no. 2, pp. 129-140, 2004.

[129] S. Jiang, B. Zhang, and D. Wei, "The elderly fall risk assessment and prediction 
based on gait analysis," in Computer and Information Technology (CIT), 2011 IEEE 11th International Conference on. IEEE, 2011, pp. 176-180.

[130] R. Hosein and M. Lord, "A study of in-shoe plantar shear in normals," Clinical biomechanics, vol. 15, no. 1, pp. 46-53, 2000.

[131] P. R. Cavanagh, J. S. Ulbrecht, and G. M. Caputo, "New developments in the biomechanics of the diabetic foot." Diabetes/metabolism research and reviews, vol. 16, pp. S6-S10, 1999.

[132] M. Yavuz, G. Botek, and B. L. Davis, "Plantar shear stress distributions: Comparing actual and predicted frictional forces at the foot-ground interface," Journal of biomechanics, vol. 40, no. 13, pp. 3045-3049, 2007.

[133] J. Apelqvist and J. Larsson, "What is the most effective way to reduce incidence of amputation in the diabetic foot?" Diabetes/metabolism research and reviews, vol. 16, no. S1, pp. S75-S83, 2000.

[134] J. E. Perry, J. O. Hall, and B. L. Davis, "Simultaneous measurement of plantar pressure and shear forces in diabetic individuals," Gait \& posture, vol. 15, no. 1, pp. 101-107, 2002 .

[135] X. Hu, J. Zhao, Y. Li, Y. He, and X. Qu, "A low cost instrumented insole to measure the center of pressure during standing," in International Conference on Mechatronics and Intelligent Robotics. Springer, 2017, pp. 293-298.

[136] S. W. Park, P. S. Das, and J. Y. Park, "Development of wearable and flexible insole type capacitive pressure sensor for continuous gait signal analysis," Organic Electronics, vol. 53, pp. 213-220, 2018.

[137] B. Nie, R. Huang, T. Yao, Y. Zhang, Y. Miao, C. Liu, J. Liu, and X. Chen, 
"Textile-based wireless pressure sensor array for human-interactive sensing," Advanced Functional Materials, p. 1808786, 2019.

[138] S. Stassi, G. Canavese, V. Cauda, C. Fallauto, S. Corbellini, P. Motto, D. Demarchi, and C. F. Pirri, "Wearable and flexible pedobarographic insole for continuous pressure monitoring," in SENSORS, 2013 IEEE. IEEE, 2013, pp. $1-4$.

[139] G. Zhu, P. Bai, J. Chen, and Z. L. Wang, "Power-generating shoe insole based on triboelectric nanogenerators for self-powered consumer electronics," Nano Energy, vol. 2, no. 5, pp. 688-692, 2013.

[140] F. Baptista, D. Budoya, V. Almeida, and J. Ulson, "An experimental study on the effect of temperature on piezoelectric sensors for impedance-based structural health monitoring," Sensors, vol. 14, no. 1, pp. 1208-1227, 2014.

[141] C.-S. Liu, G. CHOU, X. Liang, P. G. Reinhall, and W.-C. Wang, "Design of a multi-layered optical bend loss sensor for pressure and shear sensing," in Proc. of SPIE Vol, vol. 6532, 2007, pp. $65321 \mathrm{P}-1$.

[142] W. Soetanto, N. T. Nguyen, and W.-C. Wang, "Fiber optic plantar pressure/shear sensor," in Proc. SPIE, vol. 7984, 2011, p. 79840Z.

[143] C.-T. Changf, C. shih Liue, W. Soetantoc, and W.-C. Wanga, "A platform based foot pressure/shear sensor," in Proc. of SPIE Vol, vol. 8348, 2012, pp. 83 481U-1.

[144] S. Pirozzi, "Multi-point force sensor based on crossed optical fibers," Sensors and Actuators A: Physical, vol. 183, pp. 1-10, 2012.

[145] J.-C. Kim, J. Lee, K.-S. Kim, and S. Kim, "A tri-axial force sensor based on light 
intensity modulation for detecting ground reaction force," in Robotics (ISR), 2013 44th International Symposium on. IEEE, 2013, pp. 1-5.

[146] B. Najafi, H. Mohseni, G. S. Grewal, T. K. Talal, R. A. Menzies, and D. G. Armstrong, "An optical-fiber-based smart textile (smart socks) to manage biomechanical risk factors associated with diabetic foot amputation," Journal of Diabetes Science and Technology, pp. 668-677, 2017.

[147] R. Suresh, S. Bhalla, J. Hao, and C. Singh, "Development of a high resolution plantar pressure monitoring pad based on fiber Bragg grating (FBG) sensors," Technology and Health Care, vol. 23, no. 6, pp. 785-794, 2015.

[148] S. De Rossi, N. Vitiello, T. Lenzi, R. Ronsse, B. Koopman, A. Persichetti, F. Giovacchini, F. Vecchi, A. Ijspeert, H. van der Kooij et al., "Soft artificial tactile sensors for the measurement of human-robot interaction in the rehabilitation of the lower limb," in Engineering in Medicine and Biology Society (EMBC), 2010 Annual International Conference of the IEEE. IEEE, 2010, pp. 1279-1282.

[149] A. Tar and G. Cserey, "Development of a low cost 3d optical compliant tactile force sensor," in Advanced Intelligent Mechatronics (AIM), 2011 IEEE/ASME International Conference on. IEEE, 2011, pp. 236-240.

[150] G. De Maria, C. Natale, and S. Pirozzi, "Force/tactile sensor for robotic applications," Sensors and Actuators A: Physical, vol. 175, pp. 60-72, 2012.

[151] Y. Yamada, T. Morizono, Y. Umetani, and H. Takahashi, "Highly soft viscoelastic robot skin with a contact object-location-sensing capability," IEEE Transactions on Industrial electronics, vol. 52, no. 4, pp. 960-968, 2005.

[152] C. Melchiorri, L. Moriello, G. Palli, and U. Scarcia, "A new force/torque sensor 
for robotic applications based on optoelectronic components," in Robotics and Automation (ICRA), 2014 IEEE International Conference on. IEEE, 2014, pp. 6408-6413.

[153] C. Miyata, K. Chisholm, J. Baba, and M. Ahmadi, "A limb compliant sensing strategy for robot collision reaction," IEEE/ASME Transactions on Mechatronics, vol. 21, no. 2, pp. 674-682, 2016.

[154] S. Wattanasarn, K. Noda, K. Matsumoto, and I. Shimoyama, "3d flexible tactile sensor using electromagnetic induction coils," in Micro Electro Mechanical Systems (MEMS), 2012 IEEE 25th International Conference on. IEEE, 2012, pp. $488-491$.

[155] L. Shu, T. Hua, Y. Wang, Q. Li, D. D. Feng, and X. Tao, "In-shoe plantar pressure measurement and analysis system based on fabric pressure sensing array," IEEE Transactions on Information Technology in Biomedicine, vol. 14, no. 3, pp. 767-775, 2010.

[156] D. S. Chathuranga, Z. Wang, Y. Noh, T. Nanayakkara, and S. Hirai, "Disposable soft 3 axis force sensor for biomedical applications," in Engineering in Medicine and Biology Society (EMBC), 2015 37th Annual International Conference of the IEEE. IEEE, 2015, pp. 5521-5524.

[157] D. Hong, C. Li, and J. Jeong, "A crosstalk compensation of a multi-axis forcetorque sensor based on the least squares method using labview," in Computational and Information Sciences (ICCIS), 2012 Fourth International Conference on. IEEE, 2012, pp. 1127-1130.

[158] W. Zhang, K. B. Lua, K. A. S. Van Tien Truong, K. A. Senthil, T. T. Lim, K. S. 
Yeo, and G. Zhou, "Design and characterization of a novel t-shaped multi-axis piezoresistive force/moment sensor," IEEE Sensors Journal, vol. 16, no. 11, pp. 4198-4210, 2016.

[159] Y. Sun, Y. Liu, T. Zou, M. Jin, and H. Liu, "Design and optimization of a novel six-axis force/torque sensor for space robot," Measurement, vol. 65, pp. 135-148, 2015 .

[160] Y. Zhu, "Distillation column identification for control using wiener model," in American Control Conference, 1999. Proceedings of the 1999, vol. 5. IEEE, 1999, pp. 3462-3466.

[161] N. Patcharaprakiti, K. Kirtikara, D. Chenvidhya, V. Monyakul, and B. Muenpinij, "Modeling of single phase inverter of photovoltaic system using system identification," in Computer and Network Technology (ICCNT), 2010 Second International Conference on. IEEE, 2010, pp. 462-466.

[162] R. Abbasi-Asl, R. Khorsandi, and B. Vosooghi-Vahdat, "Hammerstein-wiener model: A new approach to the estimation of formal neural information," Basic and Clinical Neuroscience, vol. 3, no. 4, pp. 45-51, 2012.

[163] S. I. Biagiola and J. L. Figueroa, "Wiener and Hammerstein uncertain models identification," Mathematics and Computers in Simulation, vol. 79, no. 11, pp. 3296-3313, 2009.

[164] P. Kumar, C. Potluri, A. Sebastian, S. Chiu, A. Urfer, D. S. Naidu, and M. P. Schoen, "An adaptive multi sensor data fusion with hybrid nonlinear arx and wiener-hammerstein models for skeletal muscle force estimation," in The 14th World Scientific and Engineering Academy and Society (WSEAS) International 
Conference on Systems, Corfu Island, Greece, 2010.

[165] D. Prudham and J. G. Evans, "Factors associated with falls in the elderly: A community study," Age and Ageing, vol. 10, no. 3, pp. 141-146, 1981. [Online]. Available: +http://dx.doi.org/10.1093/ageing/10.3.141

[166] M. Tinetti, M. Speechley, and S. Ginter, "Risk factors for falls among elderly persons living in the community," New England Journal of Medicine, vol. 319, no. 26, pp. 1701-1707, 1988, pMID: 3205267. [Online]. Available: http://dx.doi.org/10.1056/NEJM198812293192604

[167] E. R. Burns, J. A. Stevens, and R. Lee, "The direct costs of fatal and non-fatal falls among older adultsâĂŤunited states," Journal of safety research, vol. 58, pp. 99-103, 2016.

[168] S. R. Lord and D. L. Sturnieks, "The physiology of falling: assessment and prevention strategies for older people," Journal of science and medicine in sport, vol. 8, no. 1, pp. 35-42, 2005.

[169] J. Sinclair, S. J. Hobbs, P. J. Taylor, G. Currigan, and A. Greenhalgh, "The influence of different force and pressure measuring transducers on lower extremity kinematics measured during running," Journal of Applied Biomechanics, vol. 30, no. 1, pp. 166-172, 2014.

[170] T. R. Han, N. J. Paik, and M. S. Im, "Quantification of the path of center of pressure (cop) using an f-scan in-shoe transducer," Gait and Posture, vol. 10, pp. 243-254, 1999.

[171] M. Munoz-Organero, J. Parker, L. Powell, and S. Mawson, "Assessing walking 
strategies using insole pressure sensors for stroke survivors," Sensors, vol. 16, no. $10,2016$.

[172] P. Catalfamo, D. Moser, S. Ghoussayni, and D. Ewins, "Detection of gait events using an f-scan in-shoe pressure measurement system," Gait \& posture, vol. 28, no. 3, pp. 420-426, 2008.

[173] E. Klimiec, B. Jasiewicz, J. Piekarski, K. Zaraska, P. Guzdek, and G. KoÅĆaszczyÅĎski, "Measuring of foot plantar pressureâǍ̆possible applications in quantitative analysis of human body mobility," Measurement Science and Technology, vol. 28, no. 5, p. 054008, 2017. [Online]. Available: http://stacks.iop.org/0957-0233/28/i=5/a=054008

[174] B. Saggin, D. Scaccabarozzi, and M. Tarabini, "Metrological performances of a plantar pressure measurement system," IEEE Transactions on Instrumentation and Measurement, vol. 62, no. 4, pp. 766-776, 2013.

[175] Y.-F. Zhang, C.-Y. Hong, R. Ahmed, and Z. Ahmed, "A fiber Bragg grating based sensing platform fabricated by fused deposition modeling process for plantar pressure measurement," Measurement, vol. 112, pp. 74 - 79, 2017. [Online]. Available: http://www.sciencedirect.com/science/article/pii/ S0263224117305225

[176] D. T.-P. Fong, Y.-Y. Chan, Y. Hong, P. S.-H. Yung, K.-Y. Fung, and K.-M. Chan, "Estimating the complete ground reaction forces with pressure insoles in walking," Journal of Biomechanics, vol. 41, pp. 2597-2601, 2008.

[177] H. Rouhani, J. Favre, X. Crevoisier, and K. Aminian, "Ambulatory assessment of 3d ground reaction force using plantar pressure distribution," Gait and Posture, 
vol. 32, pp. 311-316, 2010.

[178] P. H. Veltink, C. Liedtke, E. Droog, and H. van der Kooij, "Ambulatory measurement of ground reaction forces," IEEE Trans. Neural Syst. Rehabil. Eng., vol. 13, no. 3, Sep 2005.

[179] T. Liu, Y. Inoue, and K. Shibata, "Wearable force sensor with parallel structure for measurement of ground-reaction force," Measurement, vol. 40, no. 6, pp. 644 - 653, 2007. [Online]. Available: http://www.sciencedirect.com/science/article/ pii/S0263224106001692

[180] O. Al-Mai, M. Ahmadi, and J. Albert, "A compliant 3-axis fiber-optic force sensor for biomechanical measurement," IEEE Sensors Journal, Sep 2017.

[181] T. Liu, Y. Inoue, K. Shibata, and K. Shiojima, "A mobile force plate and three-dimensional motion analysis system for three-dimensional gait assessment," IEEE Sensors Journal, vol. 12, no. 5, pp. 1461-1467, 2012.

[182] T. Liu, Y. Inoue, and K. Shibata, "A wearable ground reaction force sensor system and its application to the measurement of extrinsic gait variability," Sensors, vol. 10, no. 11, pp. 10 240-10 255, 2010. [Online]. Available: http://www.mdpi.com/1424-8220/10/11/10240

[183] Z. Li, Y.-Y. Liang, L. Wang, J. Sheng, and S.-J. Ma, "Reliability and validity of center of pressure measures for balance assessment in older adults," Journal of Physical Therapy Science, vol. 28, no. 4, 2016.

[184] L. E. C. Lizama, M. Pijnappels, N. P. Reeves, S. M. Verschueren, and J. H. van Dieën, "Centre of pressure or centre of mass feedback in mediolateral balance assessment," Journal of biomechanics, vol. 48, no. 3, pp. 539-543, 2015. 
[185] T. Liu, Y. Inoue, and K. Shibata, "A wearable force plate system for the continuous measurement of triaxial ground reaction force in biomechanical applications," Measurement Science and Technology, vol. 21, no. 8, p. 085804, 2010. [Online]. Available: http://stacks.iop.org/0957-0233/21/i=8/a=085804

[186] T.-F. Lu, G. C. I. Lin, and J. R. He, "Neural-network-based 3d force/torque sensor calibration for robot applications," Engng Applic. Artif Intell., no. 1, 1997.

[187] J. F. Portoles and P. J. Cumpson, "A compact torsional reference device for easy, accurate and traceable AFM piconewton calibration," Nanotechnology, vol. 24, no. 33, 2013.

[188] Y. Zheng, L. Song, G. Hu, X. Cai, H. Liu, J. Ma, M. Zhao, and F. Fang, "The multi-position calibration of the stiffness for atomic-force microscope cantilevers based on vibration," Measurement Science and Technology, vol. 26, no. 5, 2015.

[189] D. Diddens, D. Reynaerts, and H. Van Brussel, "Design of a ring-shaped three-axis micro force/torque sensor," Sensors and Actuators A: Physical, vol. 46, no. 1-3, pp. 225-232, 1995.

[190] U. Seibold, B. Kubler, and G. Hirzinger, "Prototype of instrument for minimally invasive surgery with 6-axis force sensing capability," in Robotics and Automation, 2005. ICRA 2005. Proceedings of the 2005 IEEE International Conference on. IEEE, 2005, pp. 496-501.

[191] S. C. Mannsfeld, B. C. Tee, R. M. Stoltenberg, C. V. H. Chen, S. Barman, B. V. Muir, A. N. Sokolov, C. Reese, and Z. Bao, "Highly sensitive flexible pressure sensors with microstructured rubber dielectric layers," Nature materials, vol. 9, no. 10 , pp. $859-864,2010$. 
[192] C. Jacq, B. Lüthi, T. Maeder, O. Lambercy, R. Gassert, and P. Ryser, "Thick-film multi-dof force/torque sensor for wrist rehabilitation," Sensors and Actuators A: Physical, vol. 162, no. 2, pp. 361-366, 2010.

[193] G.-S. Kim, H.-M. Kim, H.-I. Kim, M.-G. Pio, H.-S. Shin, and J. Yoon, "Development of 6-axis force/moment sensor for measuring the fingers' muscular strength of human," in Industrial Electronics (ISIE), 2010 IEEE International Symposium on. IEEE, 2010, pp. 428-433.

[194] G.-S. Kim, H.-J. Shin, and J. Yoon, "Development of six-axis force/moment sensor for an intelligent foot of humanoid robot," in Control, Automation and Systems, 200\%. ICCAS'0\%. International Conference on. IEEE, 2007, pp. $2379-2384$.

[195] A. Valizadeh, A. Akbarzadeh, and M. H. T. Heravi, "Effect of structural design parameters of a six-axis force/torque sensor using full factorial design," in Robotics and Mechatronics (ICROM), 2015 3rd RSI International Conference on. IEEE, 2015, pp. 789-793.

[196] Y. Sun, Y. Liu, M. Jin, and H. Liu, "Design of a novel six-axis force/torque sensor based on strain gauges by finite element method," in Intelligent Control and Automation (WCICA), 2014 11th World Congress on. IEEE, 2014, pp. $3387-3392$.

[197] T. Mukai, S. Hirano, and Y. Kato, "Fast and accurate tactile sensor system for a human-interactive robot," in Sensors: Focus on Tactile Force and Stress Sensors. InTech, 2008.

[198] Y. Wang, G. Zuo, X. Chen, and L. Liu, "Strain analysis of six-axis force/torque 
sensors based on analytical method," IEEE Sensors Journal, vol. 17, no. 14, pp. 4394-4404, 2017.

[199] Q. Liang, D. Zhang, Q. Song, Y. Ge, H. Cao, and Y. Ge, "Design and fabrication of a six-dimensional wrist force/torque sensor based on e-type membranes compared to cross beams," Measurement, vol. 43, no. 10, pp. 1702-1719, 2010.

[200] M.-K. Kang, S. Lee, and J.-H. Kim, "Shape optimization of a mechanically decoupled six-axis force/torque sensor," Sensors and Actuators A: Physical, vol. 209, pp. 41-51, 2014.

[201] Y. Sun, Y. Liu, M. Jin, and H. Liu, "Design and optimization of a novel six-axis force/torque sensor with good isotropy and high sensitivity," in 2013 IEEE International Conference on Robotics and Biomimetics (ROBIO). IEEE, 2013, pp. 631-638.

[202] P. H. Veltink, C. Liedtke, E. Droog, and H. van der Kooij, "Ambulatory measurement of ground reaction forces," IEEE Transactions on Neural Systems and Rehabilitation Engineering, vol. 13, no. 3, pp. 423-427, 2005.

[203] H. M. Schepers, E. H. Van Asseldonk, J. H. Buurke, and P. H. Veltink, "Ambulatory estimation of center of mass displacement during walking," IEEE Transactions on Biomedical Engineering, vol. 56, no. 4, pp. 1189-1195, 2009.

[204] S. Somlor, A. Schmitz, R. Hartanto, and S. Sugano, "A prototype force sensing unit for a capacitive-type force-torque sensor," in System Integration (SII), 2014 IEEE/SICE International Symposium on. IEEE, 2014, pp. 684-689.

[205] D. Alveringh, R. A. Brookhuis, R. J. Wiegerink, and G. J. Krijnen, "A large range multi-axis capacitive force/torque sensor realized in a single SOI wafer," in 
2014 IEEE 27th International Conference on Micro Electro Mechanical Systems (MEMS). IEEE, 2014, pp. 680-683.

[206] D. Kim, C. H. Lee, B. C. Kim, D. H. Lee, H. S. Lee, C. T. Nguyen, U. K. Kim, T. D. Nguyen, H. Moon, J. C. Koo et al., "Six-axis capacitive force/torque sensor based on dielectric elastomer," in Electroactive Polymer Actuators and Devices (EAPAD) 2013, vol. 8687. International Society for Optics and Photonics, 2013, p. $86872 \mathrm{~J}$.

[207] R. A. Brookhuis, R. J. Wiegerink, T. S. Lammerink, M. J. de Boer, K. Ma, and M. Elwenspoek, "Scalable six-axis force-torque sensor with a large range for biomechanical applications," in Micro Electro Mechanical Systems (MEMS), 2012 IEEE 25th International Conference on. IEEE, 2012, pp. 595-598.

[208] R. A. Brookhuis, H. Droogendijk, M. J. de Boer, R. G. Sanders, T. S. Lammerink, R. J. Wiegerink, and G. J. Krijnen, "Six-axis force-torque sensor with a large range for biomechanical applications," Journal of micromechanics and microengineering, vol. 24, no. 3, p. 035015, 2014.

[209] A. Nakai, Y. Morishita, K. Matsumoto, and I. Shimoyama, "6-axis force-torque sensor chip composed of 16 piezoresistive beams," in Micro Electro Mechanical Systems (MEMS), 2015 28th IEEE International Conference on. IEEE, 2015, pp. $730-731$.

[210] A. Nakai, K. Kuwana, K. Saito, T. Dohi, A. Kumagai, and I. Shimoyama, "Mems 6-axis force-torque sensor attached to the tip of grasping forceps for identification of tumor in thoracoscopic surgery," in Micro Electro Mechanical Systems (MEMS), 2017 IEEE 30th International Conference on. IEEE, 2017, pp. 546-548. 
[211] Y.-J. Li, B.-Y. Sun, J. Zhang, M. Qian, and Z.-Y. Jia, "A novel parallel piezoelectric six-axis heavy force/torque sensor," Measurement, vol. 42, no. 5, pp. 730-736, 2009.

[212] Y.-j. Li, G.-c. Wang, J. Zhang, and Z.-y. Jia, "Dynamic characteristics of piezoelectric six-dimensional heavy force/moment sensor for large-load robotic manipulator," Measurement, vol. 45, no. 5, pp. 1114-1125, 2012.

[213] D. Castaño-Cano, M. Grossard, and A. Hubert, "Multi-axis force sensing using a resonant composite piezoelectric plate: model and experiments," Smart Materials and Structures, vol. 24, no. 5, p. 055019, 2015.

[214] D. M. Vogt, Y.-L. Park, and R. J. Wood, "Design and characterization of a soft multi-axis force sensor using embedded microfluidic channels," IEEE Sensors Journal, vol. 13, no. 10, pp. 4056-4064, 2013.

[215] C. Kim and C.-H. Lee, "Development of a 6-dof FBG force-moment sensor for a haptic interface with minimally invasive robotic surgery," Journal of Mechanical Science and Technology, vol. 30, no. 8, pp. 3705-3712, 2016.

[216] E. Al-Fakih, N. A. Abu Osman, and F. R. Mahamd Adikan, "The use of fiber Bragg grating sensors in biomechanics and rehabilitation applications: the state-of-the-art and ongoing research topics," Sensors, vol. 12, no. 10, pp. 12 890-12 926, 2012.

[217] F. Beyeler, S. Muntwyler, and B. J. Nelson, "Design and calibration of a microfabricated 6-axis force-torque sensor for microrobotic applications," in Robotics and Automation, 2009. ICRA'09. IEEE International Conference on. IEEE, 2009, pp. 520-525. 
[218] D.-H. Lee, U. Kim, H. Jung, and H. R. Choi, "A capacitive-type novel six-axis force/torque sensor for robotic applications," IEEE Sensors Journal, vol. 16, no. 8, pp. 2290-2299, 2016.

[219] A. Song, J. Wu, G. Qin, and W. Huang, "A novel self-decoupled four degree-of-freedom wrist force/torque sensor," Measurement, vol. 40, no. 9-10, pp. 883-891, 2007.

[220] Z.-Y. Jia, S. Lin, and W. Liu, "Measurement method of six-axis load sharing based on the stewart platform," Measurement, vol. 43, no. 3, pp. 329-335, 2010.

[221] Z. Yao, F. Wang, W. Wang, and Y. Qin, "Neural-network-based six-axis force/torque robot sensor calibration," in 2010 International Conference on Electrical and Control Engineering. IEEE, 2010, pp. 1336-1338.

[222] T.-F. Lu, G. C. Lin, and J. R. He, "Neural-network-based 3d force/torque sensor calibration for robot applications," Engineering Applications of Artificial Intelligence, vol. 10, no. 1, pp. 87-97, 1997.

[223] H. S. Oh, G. Kang, U. Kim, J. K. Seo, W. S. You, and H. R. Choi, "Force/torque sensor calibration method by using deep-learning," in Ubiquitous Robots and Ambient Intelligence (URAI), 2017 14th International Conference on. IEEE, 2017, pp. $777-782$.

[224] F. Sinden and R. Boie, "A planar capacitive force sensor with six degrees of freedom," in Proceedings. 1986 IEEE International Conference on Robotics and Automation, vol. 3. IEEE, 1986, pp. 1806-1814.

[225] S. B. Kotsiantis, "Decision trees: a recent overview," Artificial Intelligence Review, vol. 39, no. 4, pp. 261-283, 2013. 
[226] L. Rokach and O. Z. Maimon, Data mining with decision trees: theory and applications. World scientific, 2008, vol. 69.

[227] J. Brooks, R. Wentworth, R. Youngquist, M. Tur, B. Kim, and H. Shaw, "Coherence multiplexing of fiber-optic interferometric sensors," Journal of Lightwave Technology, vol. 3, no. 5, pp. 1062-1072, 1985.

[228] J. Mlodzianowski, D. Uttamchandani, and B. Culshaw, "A simple frequency domain multiplexing system for optical point sensors," Journal of lightwave technology, vol. 5, no. 7, pp. 1002-1007, 1987.

[229] B. Moslehi, H. SHAW, J. BROOKS, and B. Y. Kim, "Time-domain addressing of remote fiberoptic interferometric sensor arrays," Journal of lightwave technology, vol. 5, no. 7, pp. 1014-1023, 1987.

[230] I. Sakai, R. Youngquist, and G. Parry, "Multiplexing of optical fiber sensors using a frequency-modulated source and gated output," Journal of lightwave technology, vol. 5, no. 7, pp. 932-940, 1987.

[231] A. D. Kersey, M. A. Davis, H. J. Patrick, M. LeBlanc, K. Koo, C. Askins, M. Putnam, and E. J. Friebele, "Fiber grating sensors," Journal of lightwave technology, vol. 15, no. 8, pp. 1442-1463, 1997.

[232] X. Wan and H. F. Taylor, "Fiber-Bragg-grating pair interferometer sensor with improved multiplexing capacity and high resolution," IEEE Photonics Technology Letters, vol. 15, no. 5, pp. 742-744, 2003.

[233] H. Liu, H. Liu, G. Peng, and P. Chu, "Strain and temperature sensor using a combination of polymer and silica fibre Bragg gratings," Optics Communications, vol. 219, no. 1-6, pp. 139-142, 2003. 
[234] L. E. Pezzin, T. R. Dillingham, E. J. MacKenzie, P. Ephraim, and P. Rossbach, "Use and satisfaction with prosthetic limb devices and related services," Archives of physical medicine and rehabilitation, vol. 85, no. 5, pp. 723-729, 2004.

[235] D. Berry, "Microprocessor prosthetic knees," Physical Medicine and Rehabilitation Clinics, vol. 17, no. 1, pp. 91-113, 2006.

[236] J. Sanders, C. Daly, and E. Burgess, "Clinical measurement of normal and shear stresses on a trans-tibial stump: characteristics of wave-form shapes during walking," Prosthetics and orthotics international, vol. 17, no. 1, pp. 38-48, 1993.

[237] M. Rossi, M. Nardello, L. Lorenzelli, and D. Brunelli, "Dual mode pressure sensing for prosthetic interfaces," in 2017 7th IEEE International Workshop on Advances in Sensors and Interfaces (IWASI). IEEE, 2017, pp. 109-114.

[238] F. Jasni, N. A. Hamzaid, A. G. A. Muthalif, Z. Zakaria, H. N. Shasmin, and S.-C. Ng, "In-socket sensory system for transfemoral amputees using piezoelectric sensors: an efficacy study," IEEE/ASME Transactions on Mechatronics, vol. 21, no. 5, pp. 2466-2476, 2016.

[239] E. Zheng, L. Wang, K. Wei, and Q. Wang, “A noncontact capacitive sensing system for recognizing locomotion modes of transtibial amputees," IEEE Transactions on Biomedical Engineering, vol. 61, no. 12, pp. 2911-2920, 2014.

[240] E. Zheng and Q. Wang, "Noncontact capacitive sensing-based locomotion transition recognition for amputees with robotic transtibial prostheses," IEEE Transactions on Neural Systems and Rehabilitation Engineering, vol. 25, no. 2, pp. $161-170,2017$.

[241] P. Laszczak, L. Jiang, D. L. Bader, D. Moser, and S. Zahedi, "Development and 
validation of a 3d-printed interfacial stress sensor for prosthetic applications," Medical engineering $\&$ physics, vol. 37, no. 1, pp. 132-137, 2015.

[242] N. A. A. Razak, N. A. A. Osman, H. Gholizadeh, and S. Ali, "Prosthetics socket that incorporates an air splint system focusing on dynamic interface pressure," Biomedical engineering online, vol. 13, no. 1, p. 108, 2014.

[243] P. Convery and A. Buis, "Socket/stump interface dynamic pressure distributions recorded during the prosthetic stance phase of gait of a trans-tibial amputee wearing a hydrocast socket," Prosthetics and Orthotics International, vol. 23, no. 2, pp. 107-112, 1999.

[244] A. Hollinger and M. M. Wanderley, "Evaluation of commercial force-sensing resistors," in Proceedings of the International Conference on New Interfaces for Musical Expression, Paris, France, 2006, pp. 4-8.

[245] A. Buis and P. Convery, "Calibration problems encountered while monitoring stump/socket interface pressures with force sensing resistors: techniques adopted to minimise inaccuracies," Prosthetics and orthotics international, vol. 21, no. 3, pp. 179-182, 1997.

[246] A. Polliack, R. Sieh, D. Craig, S. Landsberger, D. McNeil, and E. Ayyappa, "Scientific validation of two commercial pressure sensor systems for prosthetic socket fit," Prosthetics and orthotics international, vol. 24, no. 1, pp. 63-73, 2000 .

[247] N. Abu Osman, W. Spence, S. Solomonidis, J. Paul, and A. Weir, "Transducers for the determination of the pressure and shear stress distribution at the stumpâǍŤsocket interface of trans-tibial amputees," Proceedings of the Institution 
of Mechanical Engineers, Part B: Journal of Engineering Manufacture, vol. 224, no. 8, pp. 1239-1250, 2010.

[248] J. E. Sanders, D. Lain, A. J. Dralle, and R. Okumura, "Interface pressures and shear stresses at thirteen socket sites on two persons with transtibial amputation," Journal of Rehabilitation Research and Development, vol. 34, pp. 19-43, 1997.

[249] T. Maeno, T. Kawamura, and S.-C. Cheng, "Friction estimation by pressing an elastic finger-shaped sensor against a surface," IEEE Transactions on Robotics and Automation, vol. 20, no. 2, pp. 222-228, 2004.

[250] M. Ohka, Y. Mitsuya, I. Higashioka, and H. Kabeshita, "An experimental optical three-axis tactile sensor for micro-robots," Robotica, vol. 23, no. 4, pp. 457-465, 2005 .

[251] E.-S. Hwang, J.-h. Seo, and Y.-J. Kim, "A polymer-based flexible tactile sensor for both normal and shear load detections and its application for robotics," Journal of microelectromechanical systems, vol. 16, no. 3, pp. 556-563, 2007.

[252] H.-K. Lee, J. Chung, S.-I. Chang, and E. Yoon, "Normal and shear force measurement using a flexible polymer tactile sensor with embedded multiple capacitors," Journal of Microelectromechanical Systems, vol. 17, no. 4, pp. 934-942, 2008.

[253] T. Agcayazi, M. McKnight, P. Sotory, H. Huang, T. Ghosh, and A. Bozkurt, "A scalable shear and normal force sensor for prosthetic sensing," in SENSORS, 2017 IEEE. IEEE, 2017, pp. 1-3.

[254] R. A. Brookhuis, R. J. Wiegerink, T. S. Lammerink, K. Ma, and G. J. Krijnen, "Large range multi-axis fingertip force sensor," in 2013 Transducers 8 
Eurosensors XXVII: The 17th International Conference on Solid-State Sensors, Actuators and Microsystems (TRANSDUCERS 8 EUROSENSORS XXVII). IEEE, 2013, pp. 2737-2740.

[255] M. I. Tiwana, A. Shashank, S. J. Redmond, and N. H. Lovell, "Characterization of a capacitive tactile shear sensor for application in robotic and upper limb prostheses," Sensors and Actuators A: Physical, vol. 165, no. 2, pp. 164-172, 2011.

[256] J. R. Mackey and B. L. Davis, "Simultaneous shear and pressure sensor array for assessing pressure and shear at foot/ground interface," Journal of biomechanics, vol. 39, no. 15, pp. 2893-2897, 2006.

[257] M. Lord and R. Hosein, "A study of in-shoe plantar shear in patients with diabetic neuropathy," Clinical Biomechanics, vol. 15, no. 4, pp. 278-283, 2000.

[258] M. J. Mueller, D. Zou, K. L. Bohnert, L. J. Tuttle, and D. R. Sinacore, "Plantar stresses on the neuropathic foot during barefoot walking," Physical therapy, vol. 88, no. 11, pp. 1375-1384, 2008.

[259] A. Rathnayaka, W. Perera, H. Savindu, K. Madarasingha, S. Ranasinghe, H. Thuduwage, A. Kulathilaka, P. Silva, S. Jayasinghe, K. Kahaduwa et al., "A customized system to assess foot plantar pressure: A case study on calloused and normal feet," arXiv preprint arXiv:1901.05303, 2019.

[260] M. E. Fernando, R. G. Crowther, and S. Wearing, "The importance of foot pressure in diabetes," Handbook of Human Motion, pp. 759-787, 2018.

[261] M. Yavuz, A. Ersen, J. Hartos, L. A. Lavery, D. K. Wukich, G. B. Hirschman, D. G. Armstrong, M. Q. Quiben, and L. S. Adams, "Temperature as a causative 
factor in diabetic foot ulceration: A call to revisit ulcer pathomechanics," Journal of the American Podiatric Medical Association, vol. 0, 2018.

[262] R. Vaishya, "Smart insole for diabetic patient," Methodology, vol. 4, no. 7, 2018.

[263] B. Najafi, D. Armstrong, and M. Bharara, "Smart textile to predict risk of diabetic foot ulcer," Sep. 6 2018, uS Patent App. 15/760,503.

[264] E. Al-Fakih, N. Osman, A. Eshraghi, and F. Adikan, "The capability of fiber Bragg grating sensors to measure amputees trans-tibial stump/socket interface pressures," Sensors, vol. 13, no. 8, pp. 10 348-10 357, 2013.

[265] Y. Liu, H. Wang, J. Yu, and P. Li, "Selective recursive kernel learning for online identification of nonlinear systems with narx form," Journal of Process Control, vol. 20, no. 2, pp. 181-194, 2010.

[266] I. Petrovic, M. Baotic, and N. Peric, "Model structure selection for nonlinear system identification using feedforward neural networks," in Neural Networks, 2000. IJCNN 2000, Proceedings of the IEEE-INNS-ENNS International Joint Conference on, vol. 1. IEEE, 2000, pp. 53-57.

[267] Y. Chetouani, "Using arx and narx approaches for modeling and prediction of the process behavior: application to a reactor-exchanger," Asia-Pacific Journal of Chemical Engineering, vol. 3, no. 6, pp. 597-605, 2008.

[268] M. V. Kumar, S. Omkar, R. Ganguli, P. Sampath, and S. Suresh, "Identification of helicopter dynamics using recurrent neural networks and flight data," Journal of the American Helicopter Society, vol. 51, no. 2, pp. 164-174, 2006.

[269] M. M. Korjani, O. Bazzaz, and M. B. Menhaj, "Real time identification 
and control of dynamic systems using recurrent neural networks," Artificial Intelligence Review, vol. 30, no. 1-4, p. 1, 2008.

[270] A. E. Ruano, P. J. Fleming, C. Teixeira, K. RodrıGuez-Vázquez, and C. M. Fonseca, "Nonlinear identification of aircraft gas-turbine dynamics," Neurocomputing, vol. 55, no. 3-4, pp. 551-579, 2003.

[271] H. Asgari, X. Chen, M. Morini, M. Pinelli, R. Sainudiin, P. R. Spina, and M. Venturini, "Narx models for simulation of the start-up operation of a single-shaft gas turbine," Applied Thermal Engineering, vol. 93, pp. 368-376, 2016.

[272] E. Cadenas, W. Rivera, R. Campos-Amezcua, and C. Heard, "Wind speed prediction using a univariate arima model and a multivariate narx model," Energies, vol. 9, no. 2, p. 109, 2016.

[273] I. Leontaritis and S. A. Billings, "Input-output parametric models for non-linear systems part i: deterministic non-linear systems," International journal of control, vol. 41, no. 2, pp. 303-328, 1985.

[274] L. C. B. da Silva, J. L. A. Samatelo, M. E. V. Segatto, J. P. Bazzo, J. C. C. da Silva, C. Martelli, and M. J. Pontes, "Narx neural network model for strong resolution improvement in a distributed temperature sensor," Applied optics, vol. 57, no. 20, pp. 5859-5864, 2018.

[275] H. Wang and G. Song, "Innovative narx recurrent neural network model for ultra-thin shape memory alloy wire," Neurocomputing, vol. 134, pp. 289-295, 2014.

[276] E. Di Tucci, S. Manfredi, C. Sansone, and S. De Vito, "A new narx based semi 
supervised learning algorithm for pollutant estimation," in Environmental Energy and Structural Monitoring Systems (EESMS), 2014 IEEE Workshop on. IEEE, 2014, pp. $1-5$.

[277] F. A. Ruslan, A. M. Samad, Z. M. Zain, and R. Adnan, "Flood water level modeling and prediction using narx neural network: Case study at kelang river," in Signal Processing 83 its Applications (CSPA), 2014 IEEE 10th International Colloquium on. IEEE, 2014, pp. 204-207.

[278] E. Esposito, S. De Vito, M. Salvato, V. Bright, R. L. Jones, and O. Popoola, "Dynamic neural network architectures for on field stochastic calibration of indicative low cost air quality sensing systems," Sensors and Actuators B: Chemical, vol. 231, pp. 701-713, 2016.

[279] H.-L. Wei and S. A. Billings, "Model structure selection using an integrated forward orthogonal search algorithm assisted by squared correlation and mutual information," International Journal of Modelling Identification and Control, vol. 3, no. 4, pp. 341-356, 2008.

[280] Y. Ma, H. Liu, Y. Zhu, F. Wang, and Z. Luo, "The narx model-based system identification on nonlinear, rotor-bearing systems," Applied Sciences, vol. 7, no. 9, p. $911,2017$.

[281] M. H. M. Som, K. Nagamune, and S. Kawaguchi, "Performance comparison of conductive rubber-based sensor array calibration using cubic spline and back propagation neural network," International Information Institute (Tokyo). Information, vol. 18, no. 2, p. 673, 2015.

[282] — , "Performance of conductive rubber-based sensor array calibration using 
cubic spline," in 2014 IEEE International Conference on Systems, Man, and Cybernetics (SMC). IEEE, 2014, pp. 3679-3684.

[283] V. Grosu, S. Grosu, B. Vanderborght, D. Lefeber, and C. Rodriguez-Guerrero, "Multi-axis force sensor for human-robot interaction sensing in a rehabilitation robotic device," Sensors, vol. 17, no. 6, p. 1294, 2017.

[284] J. Liu, L. Zhang, Z. Fu, M. Li, J. Liu, and L. Qin, "Dynamic characteristic analysis of planar piezoelectric six-axis force/torque sensor," in 2018 10th International Conference on Intelligent Human-Machine Systems and Cybernetics (IHMSC), vol. 2. IEEE, 2018, pp. 140-145.

[285] Y. Noh, J. Bimbo, S. Sareh, H. Wurdemann, J. Fraś, D. Chathuranga, H. Liu, J. Housden, K. Althoefer, and K. Rhode, "Multi-axis force/torque sensor based on simply-supported beam and optoelectronics," Sensors, vol. 16, no. 11, p. 1936, 2016 .

[286] T. Zhang, M. Tran, and H. Huang, "Design and experimental verification of hip exoskeleton with balance capacities for walking assistance," IEEE/ASME Transactions on Mechatronics, 2018.

[287] Y. Ma, S. Xie, X. Zhang, and Y. Luo, "Hybrid calibration method for six-component force/torque transducers of wind tunnel balance based on support vector machines," Chinese Journal of Aeronautics, vol. 26, no. 3, pp. 554-562, 2013.

[288] E. Bayo and J. Stubbe, "Six-axis force sensor evaluation and a new type of optimal frame truss design for robotic applications," Journal of Robotic Systems, vol. 6, no. 2, pp. 191-208, 1989. 
[289] C. Ren, Y. Gong, F. Jia, and X. Wang, "Theoretical analysis of a six-axis force/torque sensor with overload protection for polishing robot," in 2016 23rd International Conference on Mechatronics and Machine Vision in Practice (M2VIP). IEEE, 2016, pp. 1-6.

[290] Y. B. Kim, U. Kim, D.-Y. Seok, J. So, and H. R. Choi, "A novel capacitive type torque sensor for robotic applications," in 2016 IEEE International Conference on Advanced Intelligent Mechatronics (AIM). IEEE, 2016, pp. 993-998.

[291] Y. Sun, K. Kim, R. M. Voyles, and B. J. Nelson, "Calibration of multi-axis mems force sensors using the shape from motion method," in Proceedings 2006 IEEE International Conference on Robotics and Automation, 2006. ICRA 2006. IEEE, 2006, pp. 269-274.

[292] K. Kim, Y. Sun, R. M. Voyles, and B. J. Nelson, "Calibration of multi-axis mems force sensors using the shape-from-motion method," IEEE Sensors Journal, vol. 7, no. 3, pp. 344-351, 2007.

[293] J. Huang, C. Y. Wong, D. T. Pham, Y. Wang, C. Ji, S. Su, W. Xu, Q. Liu, and Z. Zhou, "Design of a novel six-axis force/torque sensor based on optical fibre sensing for robotic applications," 2018.

[294] D. Chen, A. Song, and A. Li, "Design and calibration of a six-axis force/torque sensor with large measurement range used for the space manipulator," Procedia Engineering, vol. 99, pp. 1164-1170, 2015.

[295] R. Kumar, S. Rab, B. Pant, S. Maji, and R. Mishra, "Fea-based design studies for development of diaphragm force transducers," MAPAN, pp. 1-9, 2018.

[296] K. H. Sienko, V. V. Vichare, M. D. Balkwill, and C. Wall III, "Assessment of 
vibrotactile feedback on postural stability during pseudorandom multidirectional platform motion," IEEE Transactions on Biomedical Engineering, vol. 57, no. 4, pp. 944-952, 2010.

[297] M. R. Afzal, Y. Jan, S. Hassan, and J. Yoon, "Analysis of active haptic feedback effects on standing stability," in International Conference on Intelligent Robotics and Applications. Springer, 2013, pp. 154-164.

[298] B.-C. Lee, J. Kim, S. Chen, and K. H. Sienko, "Cell phone based balance trainer," Journal of neuroengineering and rehabilitation, vol. 9, no. 1, p. 10, 2012.

[299] J.-F. Bayouk, J. P. Boucher, and A. Leroux, "Balance training following stroke: effects of task-oriented exercises with and without altered sensory input," International Journal of Rehabilitation Research, vol. 29, no. 1, pp. 51-59, 2006.

[300] B. Lange, S. Flynn, R. Proffitt, C.-Y. Chang, and A. âĂIJSkipâĂİ Rizzo, "Development of an interactive game-based rehabilitation tool for dynamic balance training," Topics in stroke rehabilitation, vol. 17, no. 5, pp. 345-352, 2010.

[301] A. Srivastava, A. B. Taly, A. Gupta, S. Kumar, and T. Murali, "Post-stroke balance training: role of force platform with visual feedback technique," Journal of the neurological sciences, vol. 287, no. 1, pp. 89-93, 2009.

[302] W. Young, S. Ferguson, S. Brault, and C. Craig, "Assessing and training standing balance in older adults: a novel approach using the nintendo wii balance board," Gait $\mathscr{E}$ posture, vol. 33, no. 2, pp. 303-305, 2011.

[303] C. Franco, A. Fleury, P.-Y. Guméry, B. Diot, J. Demongeot, and N. Vuillerme, 
"ibalance-abf: a smartphone-based audio-biofeedback balance system," IEEE transactions on biomedical engineering, vol. 60, no. 1, pp. 211-215, 2013.

[304] M. Dozza, F. B. Horak, and L. Chiari, "Auditory biofeedback substitutes for loss of sensory information in maintaining stance," Experimental brain research, vol. 178, no. 1, pp. 37-48, 2007.

[305] L. Chiari, M. Dozza, A. Cappello, F. B. Horak, V. Macellari, and D. Giansanti, "Audio-biofeedback for balance improvement: an accelerometry-based system," IEEE transactions on biomedical engineering, vol. 52, no. 12, pp. 2108-2111, 2005.

[306] N. Vuillerme, O. Chenu, J. Demongeot, and Y. Payan, "Controlling posture using a plantar pressure-based, tongue-placed tactile biofeedback system," Experimental brain research, vol. 179, no. 3, pp. 409-414, 2007.

[307] S. E. Sihvonen, S. Sipilä, and P. A. Era, "Changes in postural balance in frail elderly women during a 4-week visual feedback training: a randomized controlled trial," Gerontology, vol. 50, no. 2, pp. 87-95, 2004.

[308] R. A. Geiger, J. B. Allen, J. O’Keefe, and R. R. Hicks, "Balance and mobility following stroke: effects of physical therapy interventions with and without biofeedback/forceplate training," Physical therapy, vol. 81, no. 4, pp. 995-1005, 2001.

[309] E. Bisson, B. Contant, H. Sveistrup, and Y. Lajoie, "Functional balance and dual-task reaction times in older adults are improved by virtual reality and biofeedback training," Cyberpsychology \& behavior, vol. 10, no. 1, pp. 16-23, 2007. 
[310] T. E. Prieto, J. Myklebust, R. Hoffmann, E. Lovett, and B. Myklebust, "Measures of postural steadiness: differences between healthy young and elderly adults," IEEE Transactions on biomedical engineering, vol. 43, no. 9, pp. 956-966, 1996.

[311] K. Seo, J. Lee, Y. Lee, T. Ha, and Y. Shim, "Fully autonomous hip exoskeleton saves metabolic cost of walking," in Robotics and Automation (ICRA), 2016 IEEE International Conference on Robotics and Automation (ICRA). IEEE, 2016, pp. 4628-4635.

[312] K. Seo, J. Lee, and Y. J. Park, "Autonomous hip exoskeleton saves metabolic cost of walking uphill," in Rehabilitation Robotics (ICORR), 2017 International Conference on Rehabilitation Robotics (ICORR). IEEE, 2017, pp. 246-251.

[313] J. Beil and T. Asfour, "New mechanism for a 3 dof exoskeleton hip joint with five revolute and two prismatic joints," in Biomedical Robotics and Biomechatronics (BioRob), 2016 6th IEEE International Conference on Biomedical Robotics and Biomechatronics (BioRob). IEEE, 2016, pp. 787-792.

[314] J. Jang, K. Kim, J. Lee, B. Lim, and Y. Shim, "Online gait task recognition algorithm for hip exoskeleton," in Intelligent Robots and Systems (IROS), 2015 IEEE/RSJ International Conference on Intelligent Robots and Systems (IROS. IEEE, 2015, pp. 5327-5332.

[315] S. O. Schrade, Y. Nager, A. R. Wu, R. Gassert, and A. Ijspeert, "Bio-inspired control of joint torque and knee stiffness in a robotic lower limb exoskeleton using a central pattern generator," in 2017 International Conference on Rehabilitation Robotics (ICORR). IEEE, 2017, pp. 1387-1394.

[316] A. Martínez, B. Lawson, and M. Goldfarb, "A controller for guiding leg movement 
during overground walking with a lower limb exoskeleton," IEEE Transactions on Robotics, 2017.

[317] R. Analia, K.-T. Song et al., "Design of assistive torque for a lower limb exoskeleton based on motion prediction," in Society of Instrument and Control Engineers of Japan (SICE), 2016 55th Annual Conference of the Society of Instrument and Control Engineers of Japan (SICE). IEEE, 2016, pp. 172-177.

[318] K. A. Witte, A. M. Fatschel, and S. H. Collins, "Design of a lightweight, tethered, torque-controlled knee exoskeleton," in Rehabilitation Robotics (ICORR), 2017 International Conference on Rehabilitation Robotics (ICORR). IEEE, 2017, pp. $1646-1653$.

[319] Z. F. Lerner, D. L. Damiano, and T. C. Bulea, "Relationship between assistive torque and knee biomechanics during exoskeleton walking in individuals with crouch gait," in Rehabilitation Robotics (ICORR), 2017 International Conference on Rehabilitation Robotics (ICORR). IEEE, 2017, pp. 491-497.

[320] H. Choi, Y. J. Park, K. Seo, J. Lee, S.-e. Lee, and Y. Shim, "A multifunctional ankle exoskeleton for mobility enhancement of gait-impaired individuals and seniors," IEEE Robotics and Automation Letters, vol. 3, no. 1, pp. 411-418, 2018.

[321] C. Meijneke, W. van Dijk, and H. van der Kooij, "Achilles: an autonomous lightweight ankle exoskeleton to provide push-off power," in 5th IEEE RAS/EMBS International Conference on Biomedical Robotics and Biomechatronics. IEEE, 2014, pp. 918-923.

[322] K. A. Witte, J. Zhang, R. W. Jackson, and S. H. Collins, "Design of two lightweight, high-bandwidth torque-controlled ankle exoskeletons," in Robotics 
and Automation (ICRA), 2015 IEEE International Conference on Robotics and Automation (ICRA). IEEE, 2015, pp. 1223-1228.

[323] M. B. Wiggin, G. S. Sawicki, and S. H. Collins, "An exoskeleton using controlled energy storage and release to aid ankle propulsion," in Rehabilitation Robotics (ICORR), 2011 IEEE International Conference on. IEEE, 2011, pp. 1-5.

[324] Z.-F. Fu and J. He, Modal analysis. Elsevier, 2001.

[325] H. A. Al-Khazali and M. R. Askari, "Calculations of frequency response functions (frf) using computer smart office software and nyquist plot under gyroscopic effect rotation," arXiv preprint arXiv:1208.3681, 2012. 
Appendices 


\section{Appendix A}

\section{Experimental Results of the light}

\section{intensity Modulation-Based, 3-Axis}

\section{Force Sensor}

\section{A.1 Experimental Validation Through An Actual Gait Measurement Scenario}

To evaluate the proposed sensor's capability to measure three-axis forces in a real gait measurement scenario, the force sensing units were tested while mounted to the floor. Both sensors were attached to a Bertec force plate (FP 4060-07-1000, Ohio, United States) as shown in Figure 1.1. The force plate has a six-axis, strain gauge-base force and torque sensor. In this experimental procedure, the sensors are around $10 \mathrm{~cm}$ apart along the $y$-axis of the force plate and are aligned to the z-axis. 


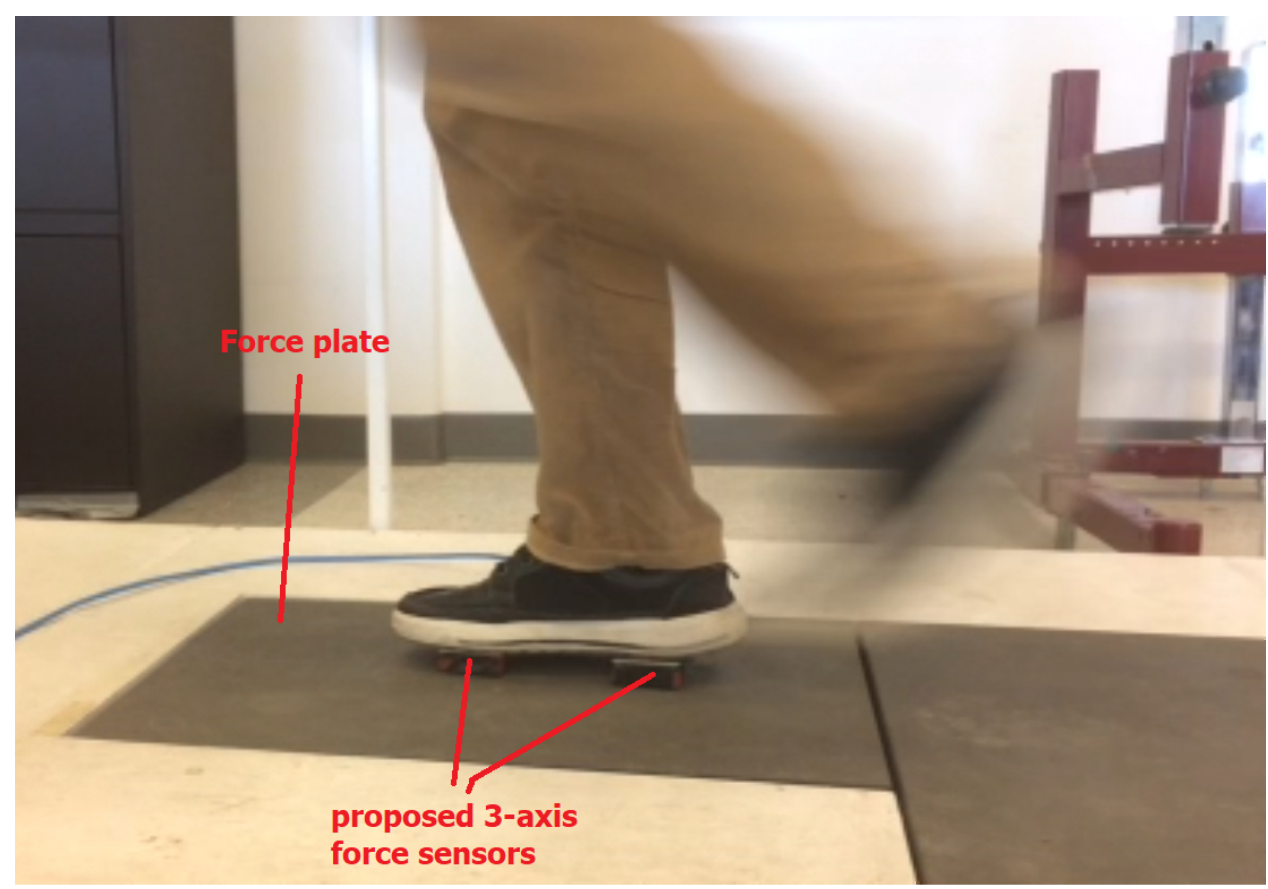

Figure 1.1: floor-mounted experiment. Two three-axial force sensors are affixed on the Bertec force plate while the subjects walking across.

Gait data is recorded as a subject stepped on the two sensors while walking across the force plate. Data is acquired using Bertec digital acquire with AM6800 digital output amplifier, and NI PCI 6229 data acquisition system from National Instrument with a sampling rate of $1000 \mathrm{~Hz}$. Sensor data is compared with force measurements obtained from the reference (force plate).

As the subject plays a role of applying force which does not change the physics of the proposed sensor. It has been found that sensor behaviour is independent from the subject weight and subject's gait speed. An actual gait measurement scenario is demonstrated instead of applying loads using MTS machine in order to illustrate the ability of the proposed sensor when it is used in one of its intended actual applications.

A high precision force measurement for each axis is achieved. Figure 1.2 shows the ground reaction force acting along the $\mathrm{x}$-axis, representing one of the shear force 


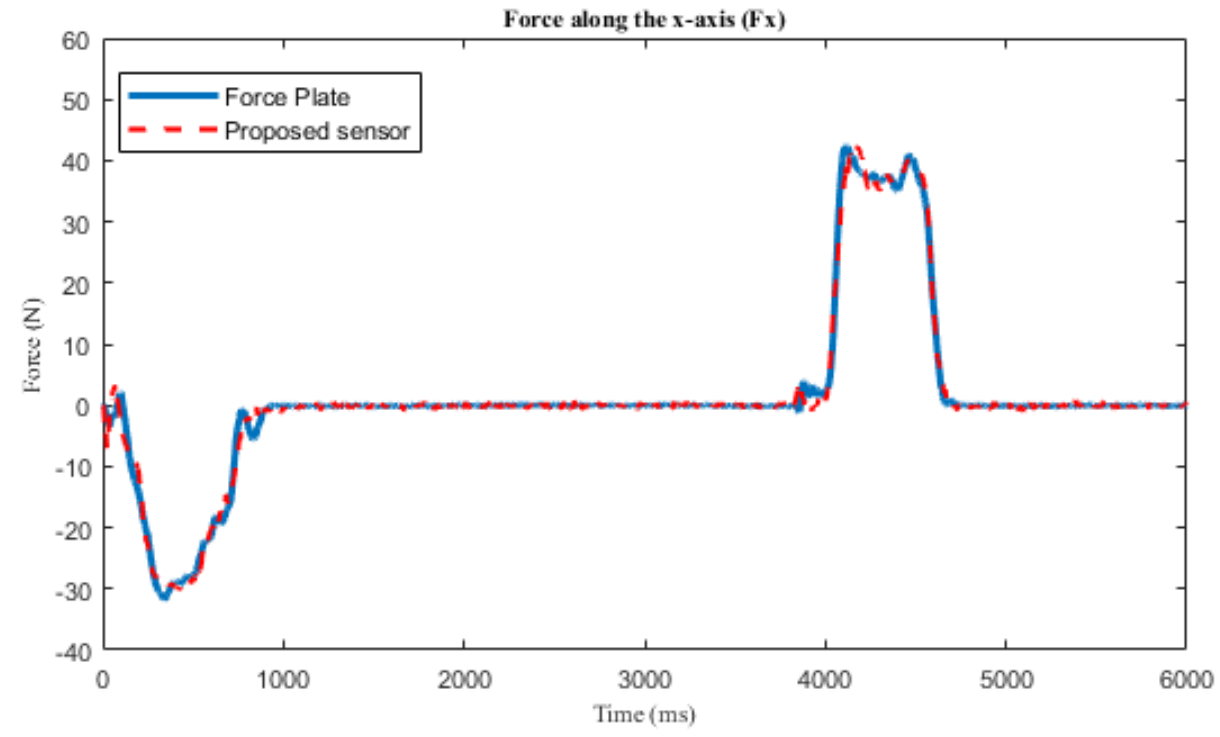

Figure 1.2: Force sensor response due to the applied shear force along the $\mathrm{x}$-axis during gait

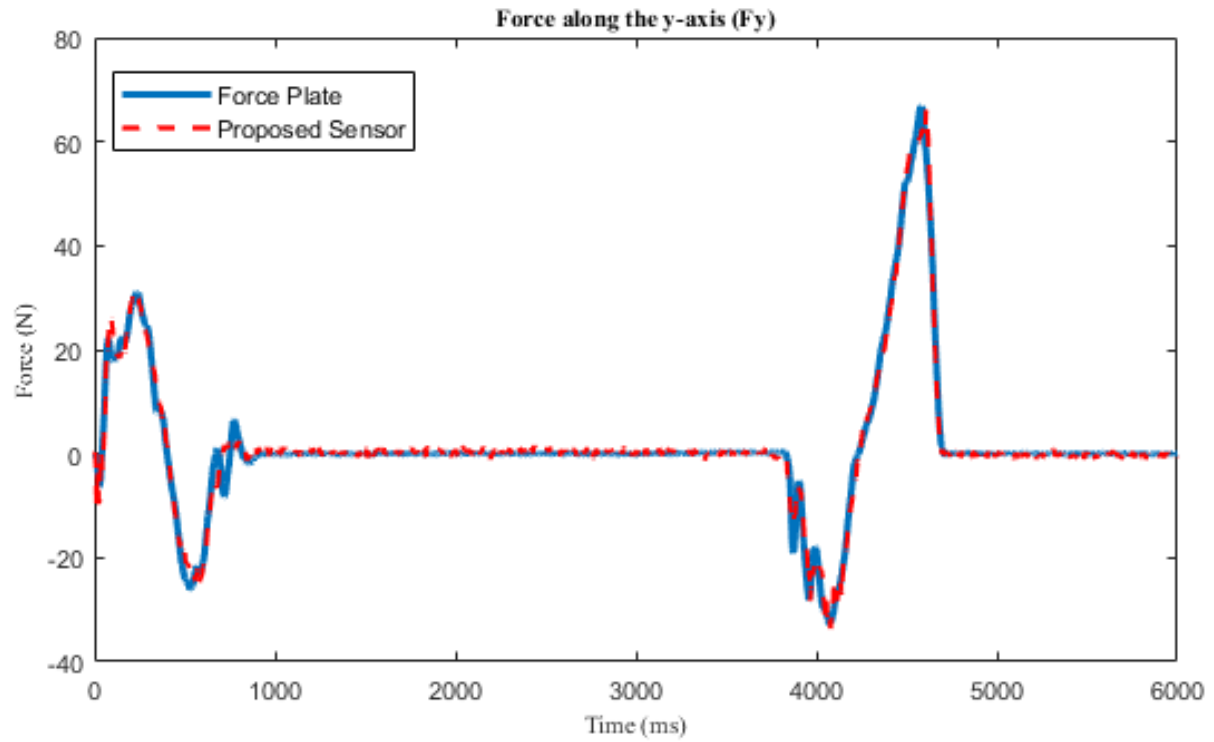

Figure 1.3: Force sensor response due to the applied shear force along the y-axis during gait 


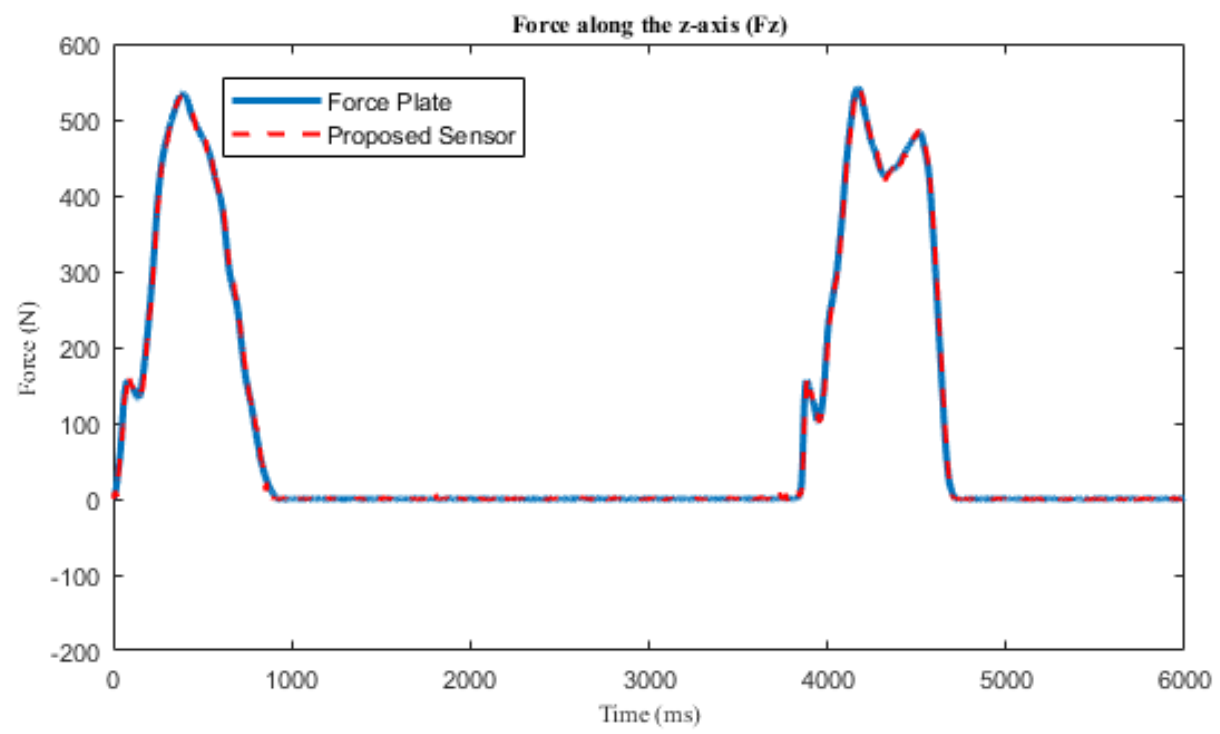

Figure 1.4: Force sensor response due to the applied normal force along the z-axis during gait

components as the root mean square error RMSE is $2.9 \%$ out of force range of about $45 \mathrm{~N}$. The other shear force component can be seen in Figure 1.3, which is the shear reaction force acting along the y-axis, exhibiting a RMSE of $2.5 \%$ out of shear force range of about 60 N. Figure 1.4 depicts the sensor response to the vertical ground reaction force acting along the z-axis which also shows an excellent performance with a RMSE of only $0.8 \%$ out of a normal force range of over $500 \mathrm{~N}$. Overall, the proposed sensor exhibits very good results during this experimental procedure. The estimated results closely match the measured values of the force obtained from the force plate. 


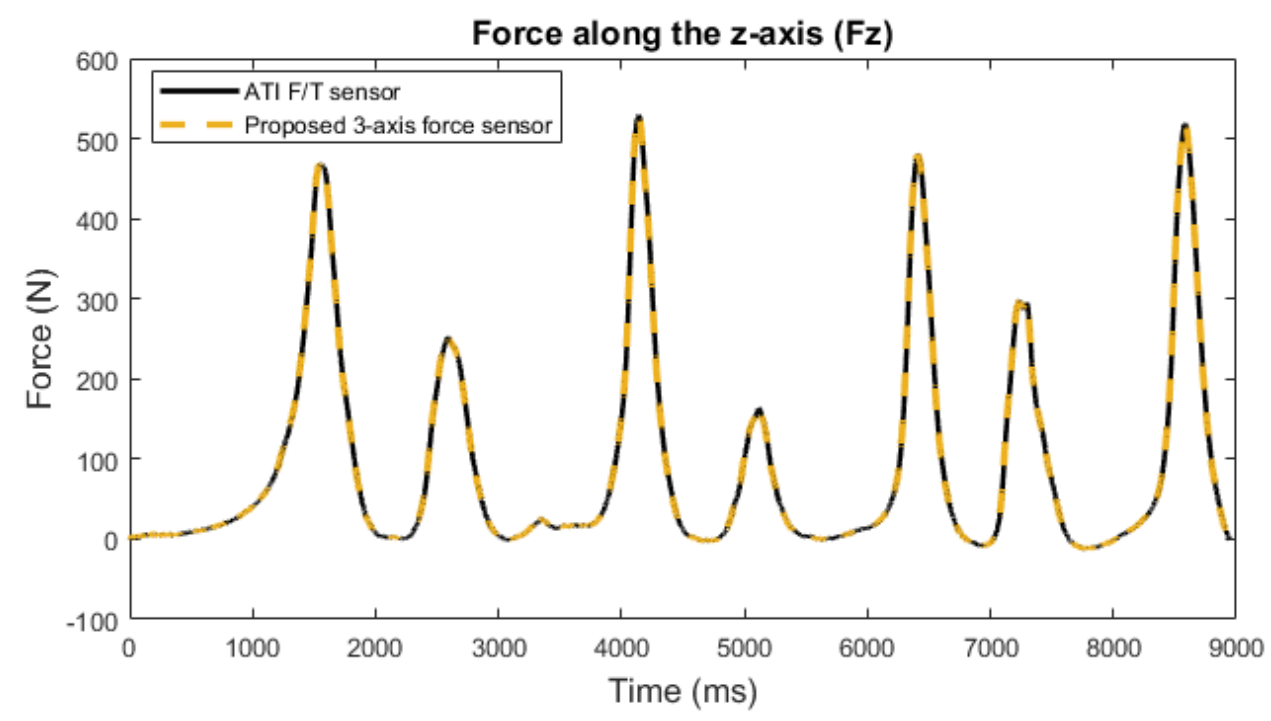

Figure 1.5: a comparison between the experimental results of the proposed 3-axis intensity based force sensor and the ATI force and torque sensor when a force is applied along the z-axis.

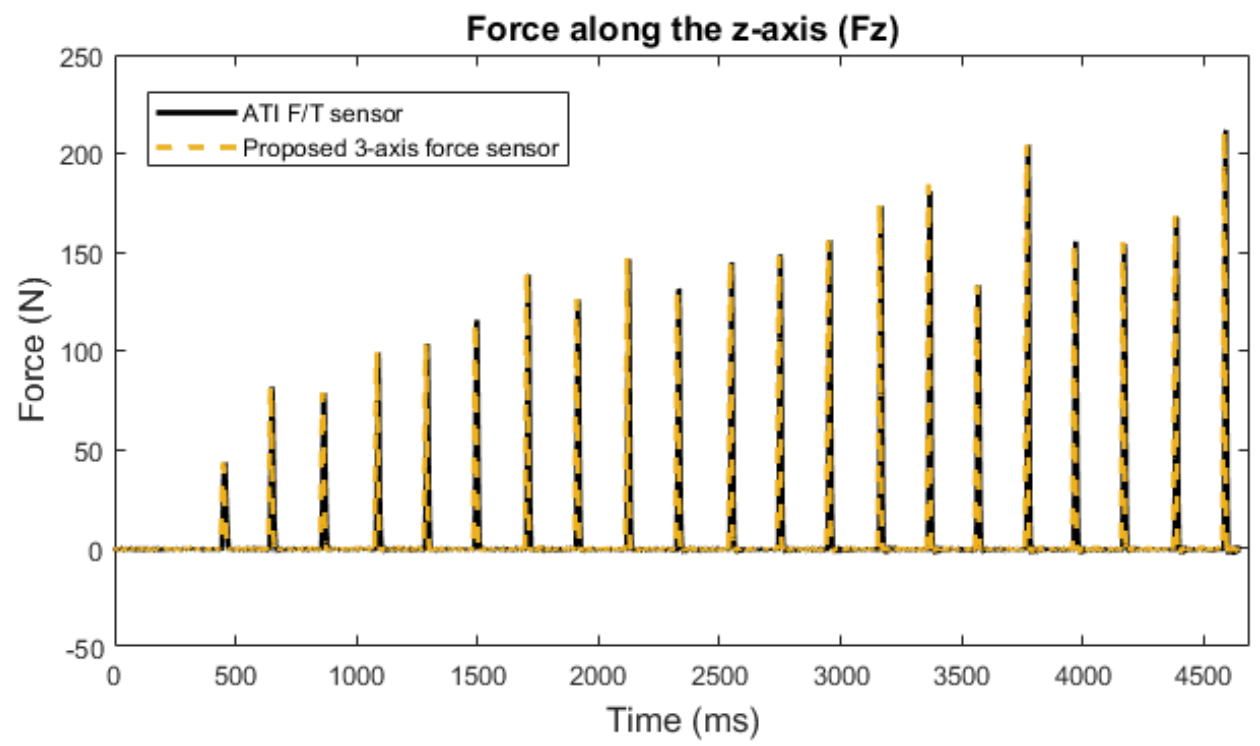

Figure 1.6: Sensor performance when a force of high of high frequency and high speed is applied. 


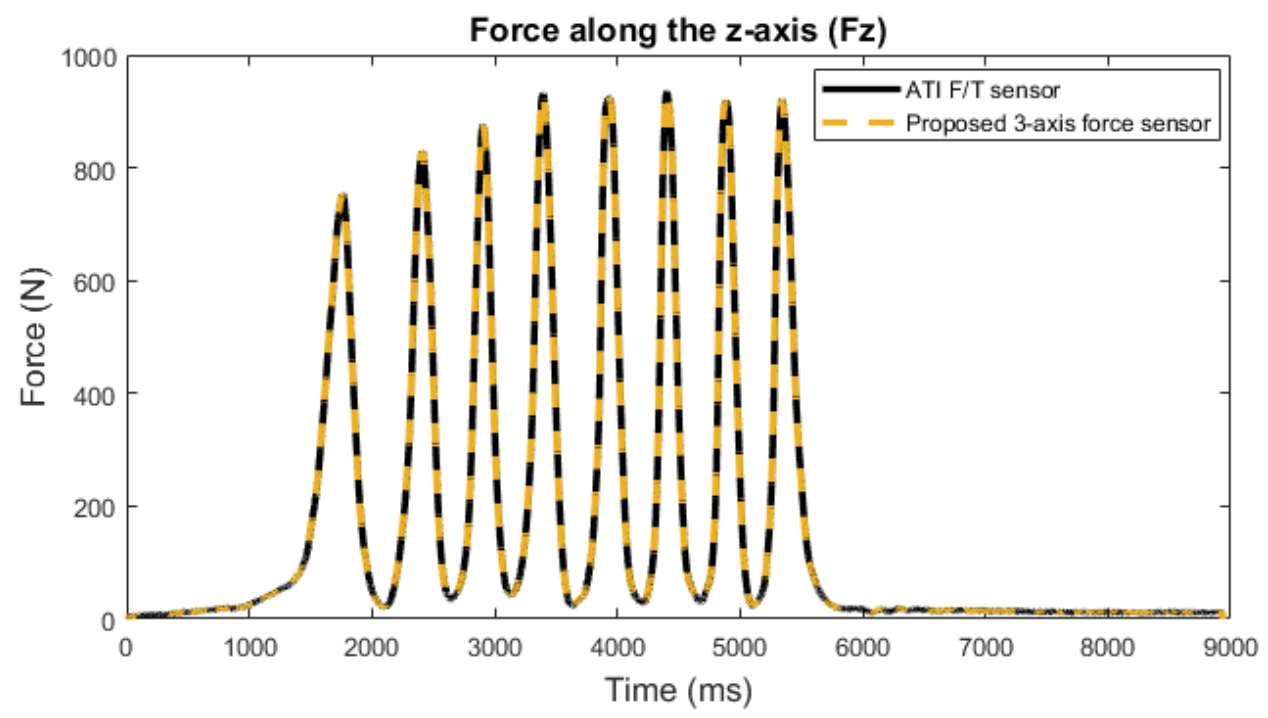

Figure 1.7: The experimental results of the propse 3-xis force sensor and the reference sensor when a large amount of normal force is applied.

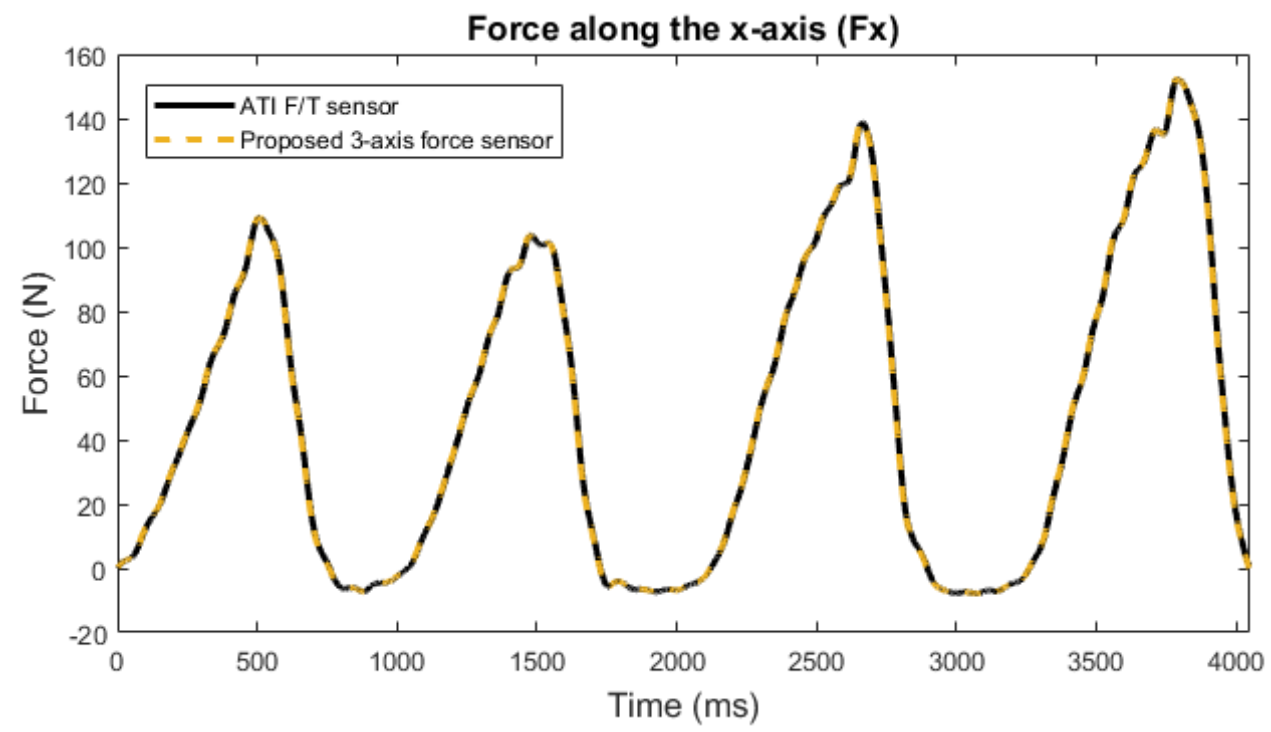

Figure 1.8: a comparison between the experimental results of the proposed 3-axis intensity based force sensor and the reference sensor in terms of shear force applied along the x-axis. 


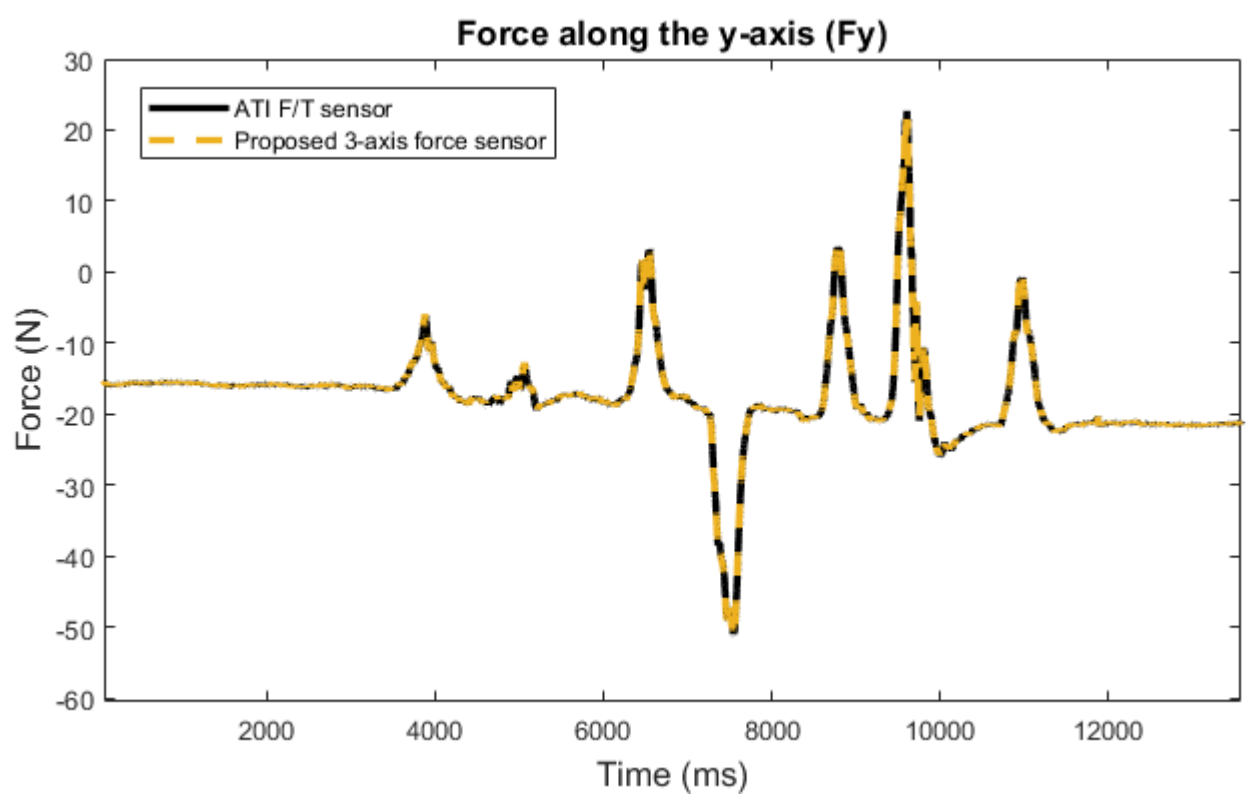

Figure 1.9: Shear force sensing element performance in a comparison to the ATI force and torque sensor. This shear force is acting along the $\mathrm{y}$-axis.

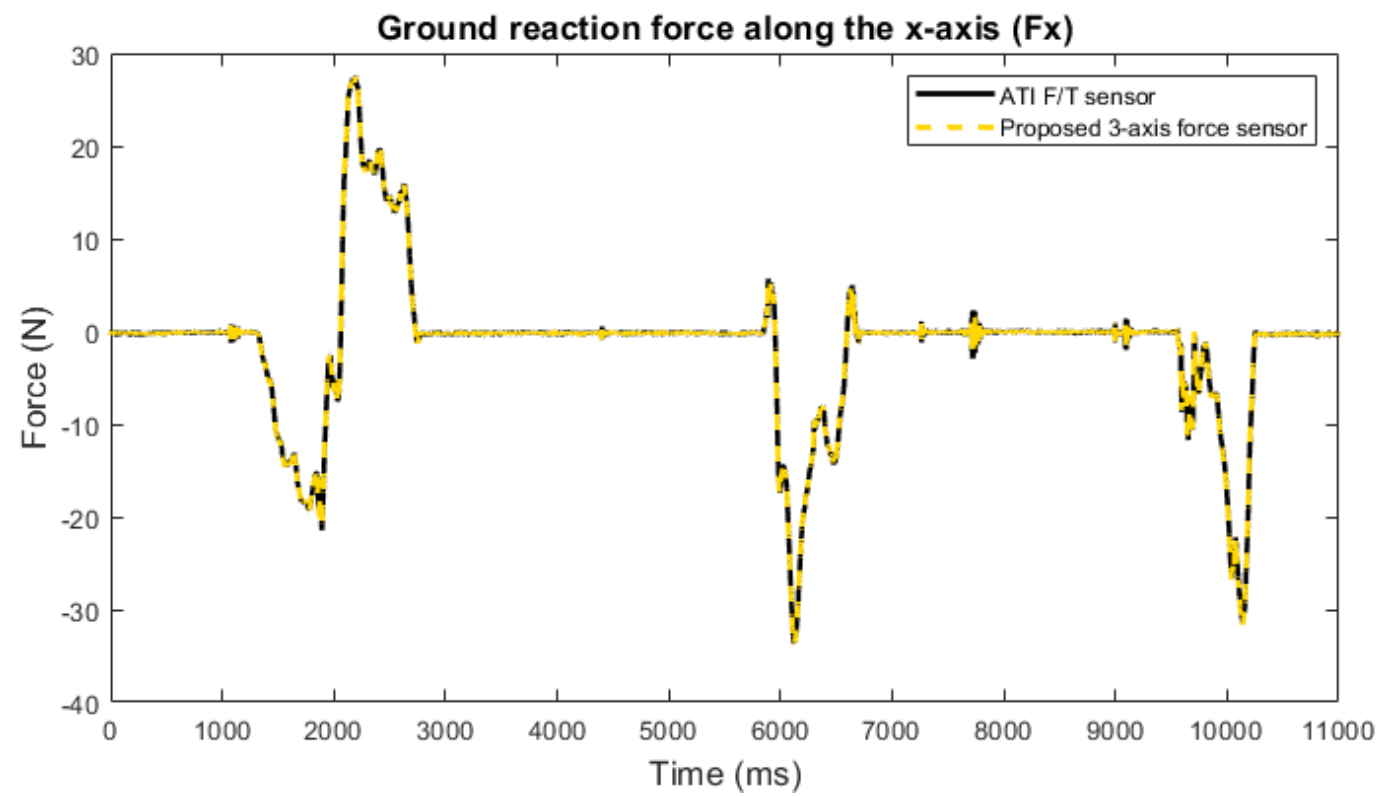

Figure 1.10: Ground reaction force measurement. The sensor is tested during gait demonstrating sensor results of the shear force applied along the $\mathrm{x}$-axis 


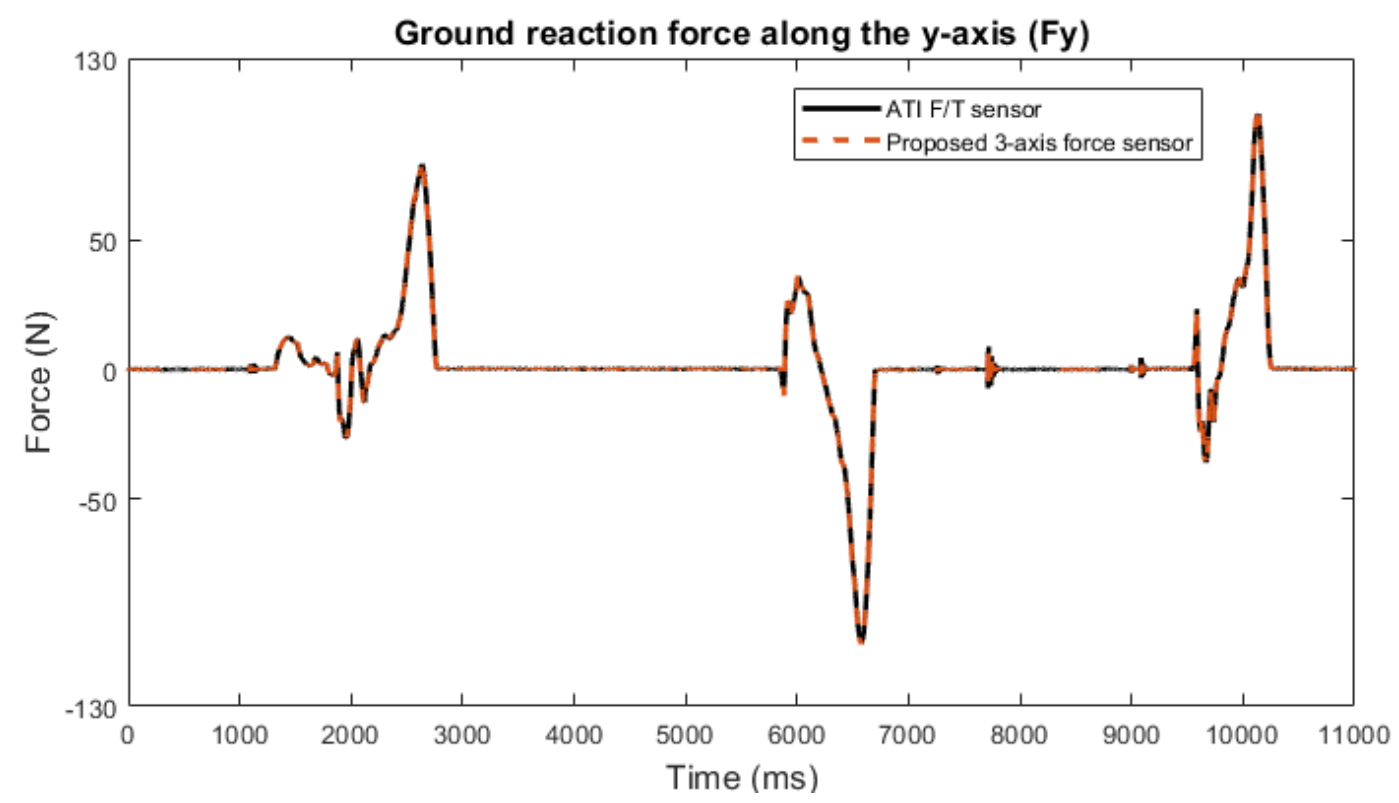

Figure 1.11: Ground reaction force measurement. The sensor is tested during gait demonstrating sensor results of the shear force applied along the y-axis

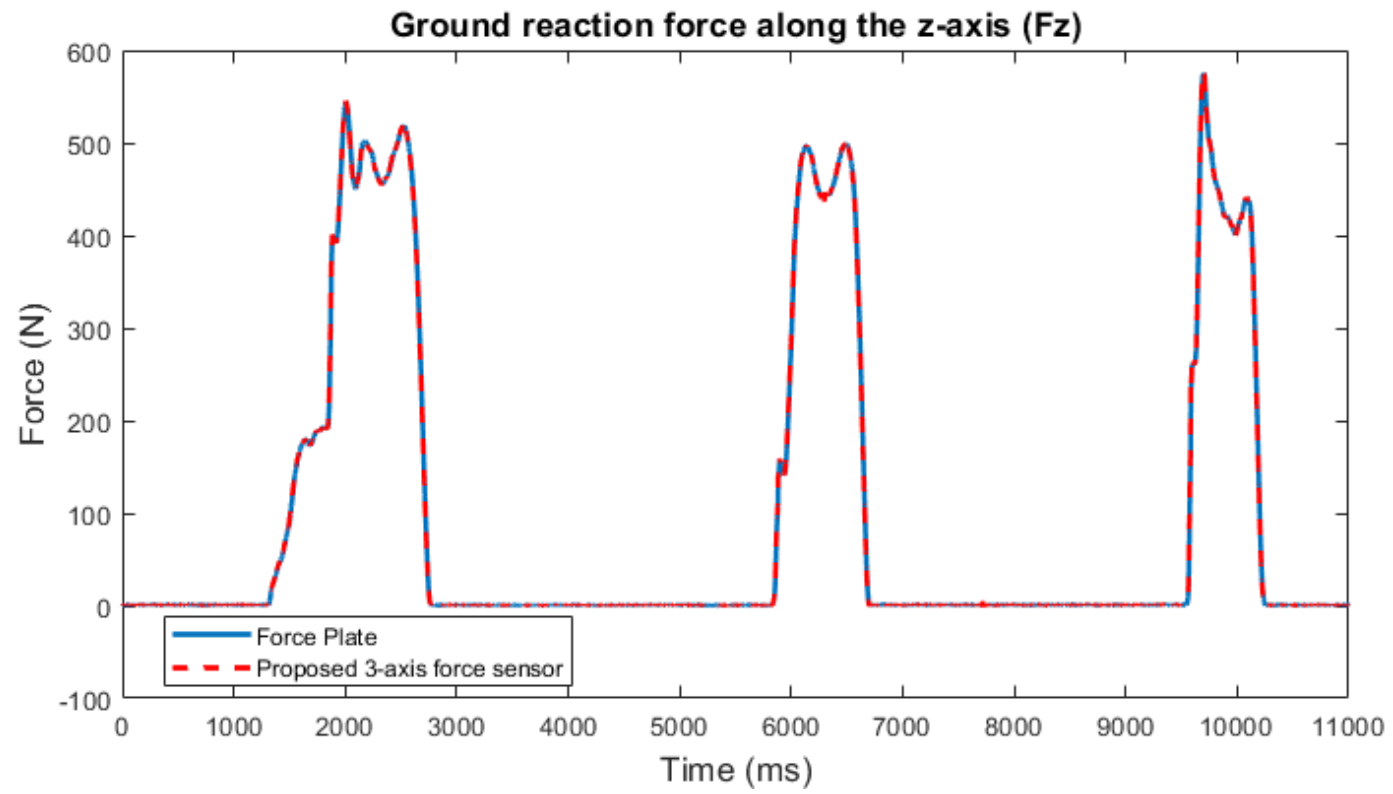

Figure 1.12: Ground reaction force measurement. The sensor is tested during gait demonstrating sensor results of the normal force applied along the z-axis 


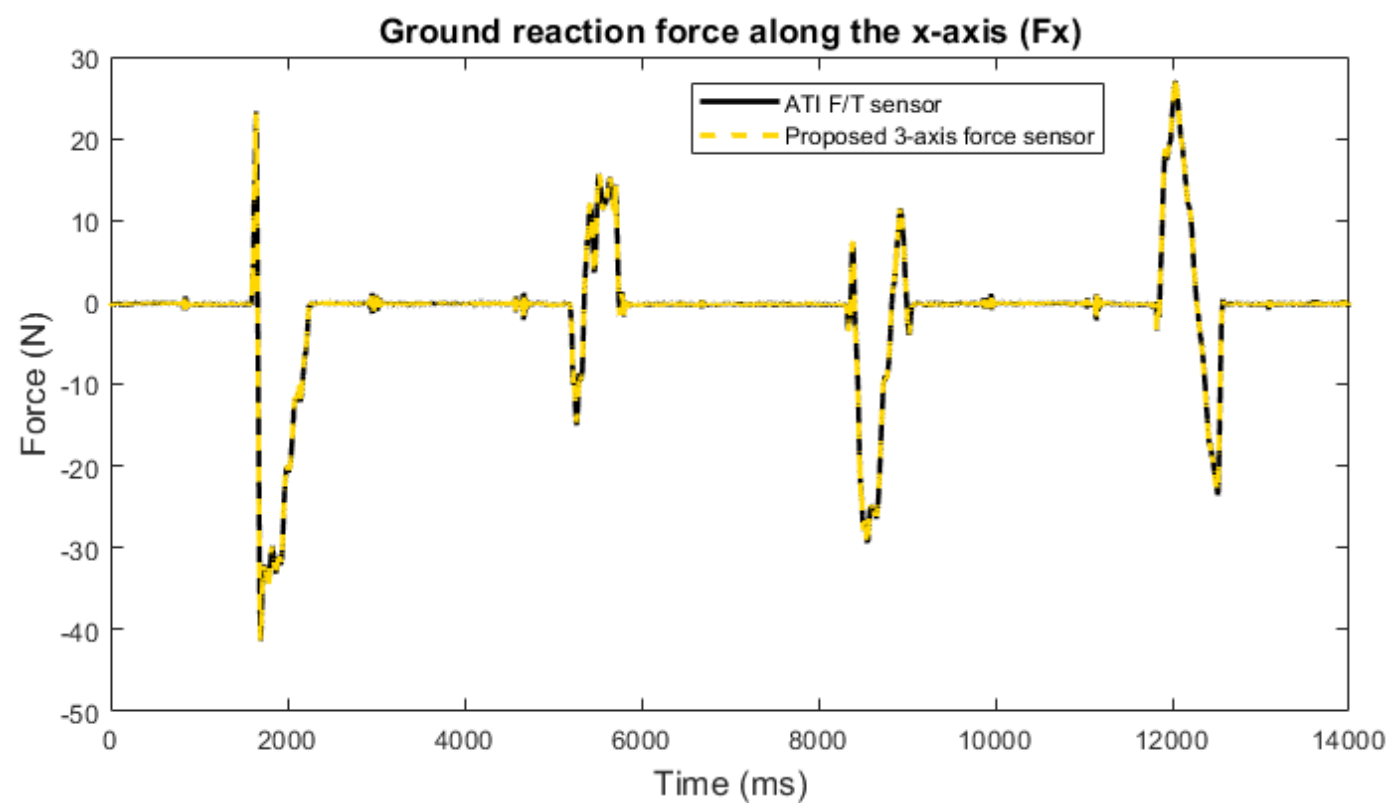

Figure 1.13: Experimental results of the proposed 3-axis force sensor during gait test presenting four steps while the shear force is applied along the $\mathrm{x}$-axis.

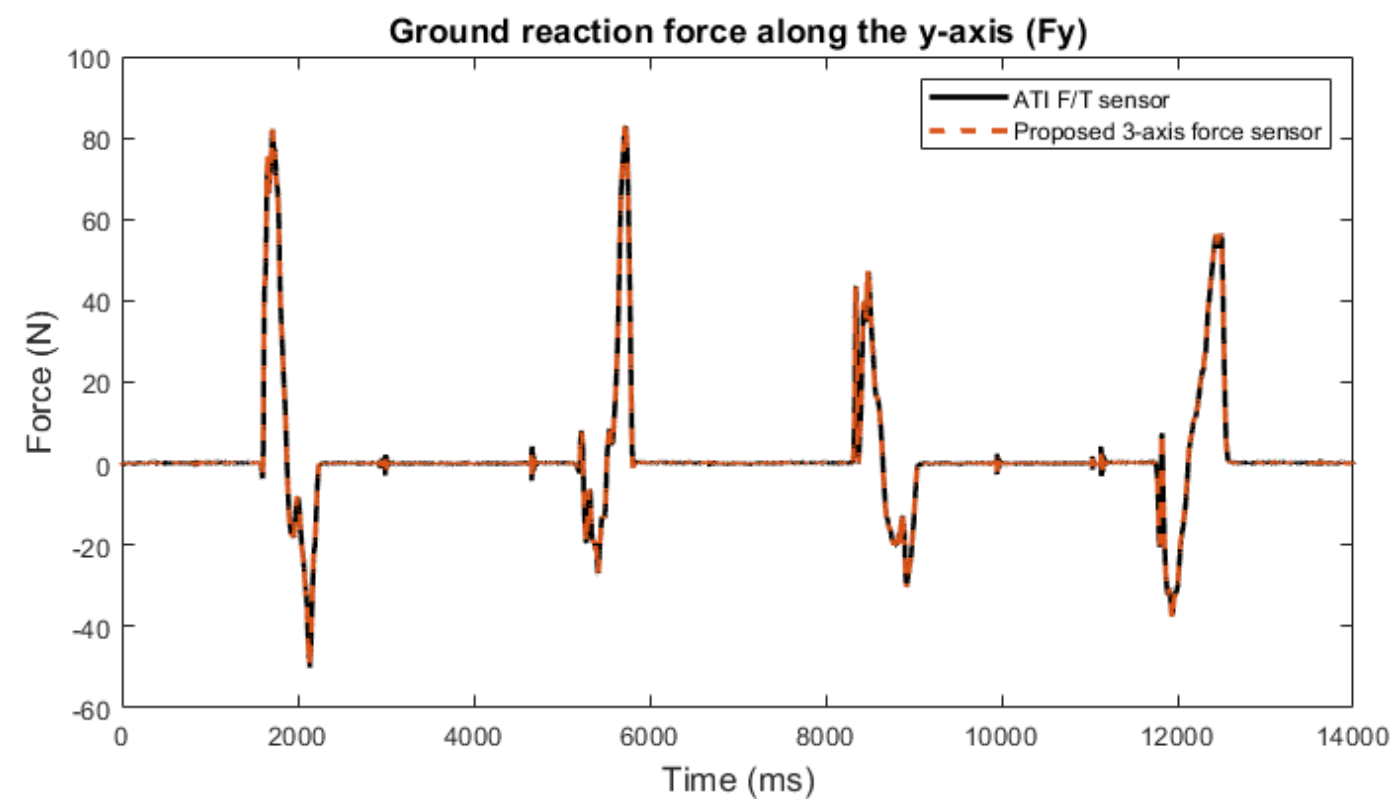

Figure 1.14: Ground reaction shear force acting along the y-axis presenting four steps of the sensor performance compared to the force plate. 


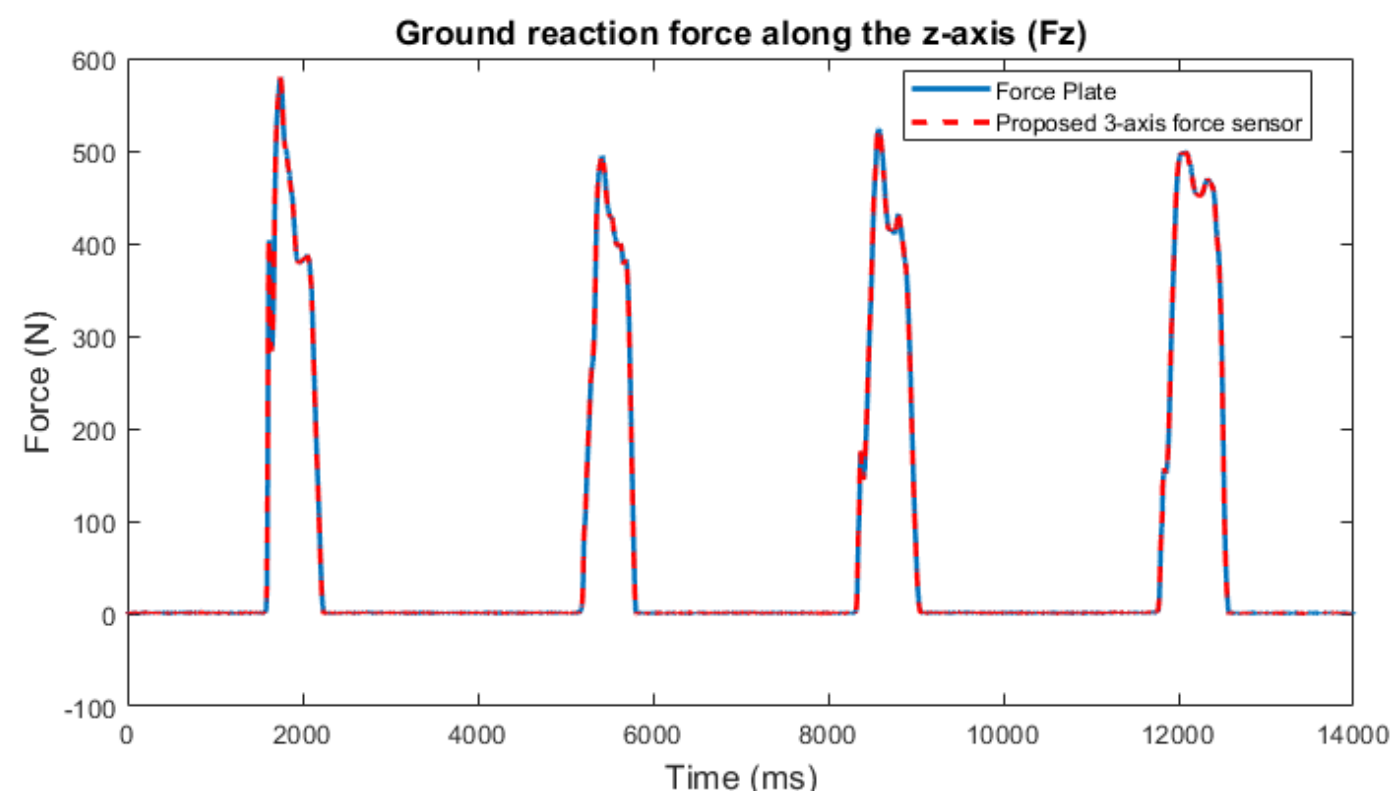

Figure 1.15: Experimental results of the proposed 3-axis force sensor during gait test showing four steps while the normal force is applied along the $\mathrm{x}$-axis

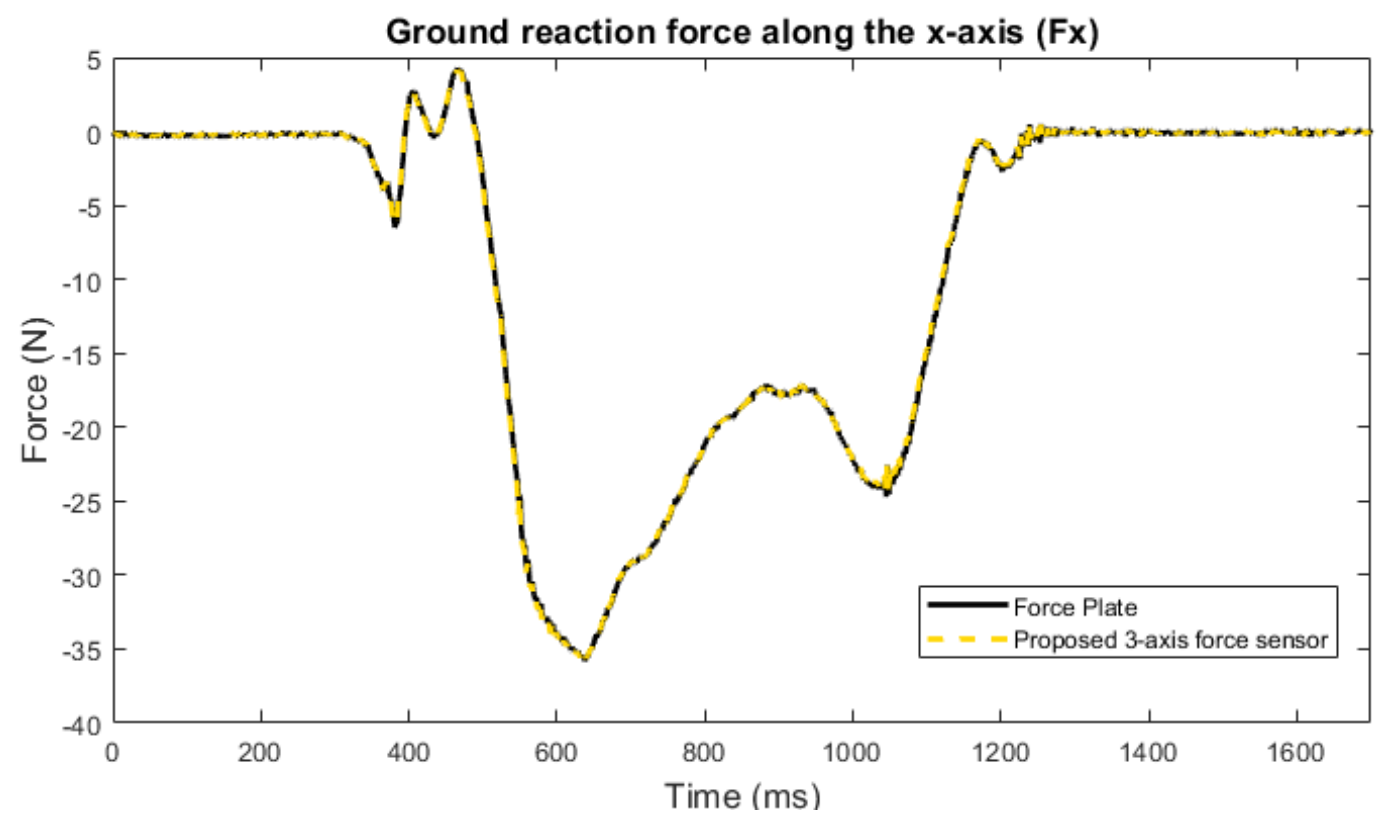

Figure 1.16: One step test of ground reaction force along the x-direction in comparison to the force plate measurements. 


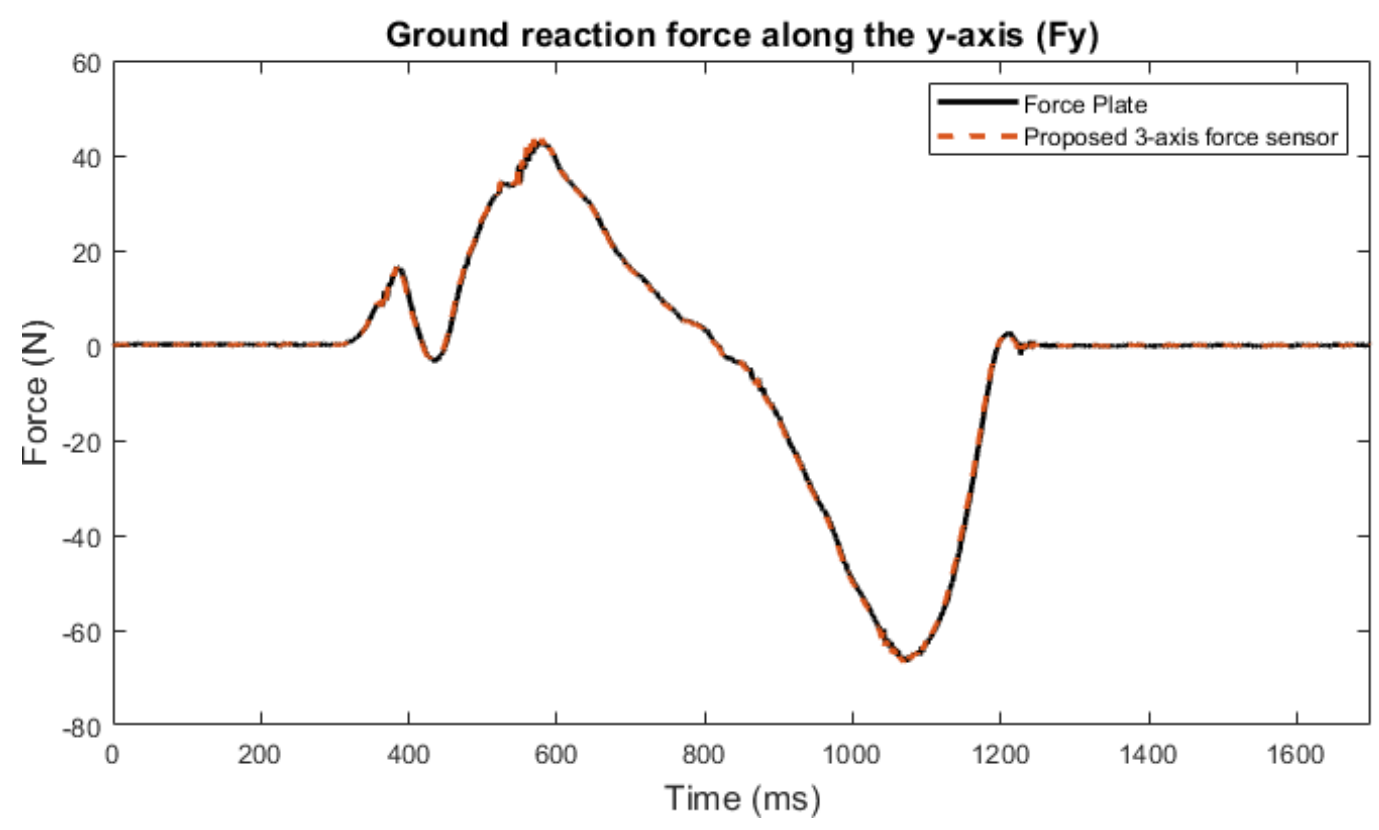

Figure 1.17: The experimental results of the proposed 3-axis force sensor in real gait measurement of the shear force of the $y$-direction.

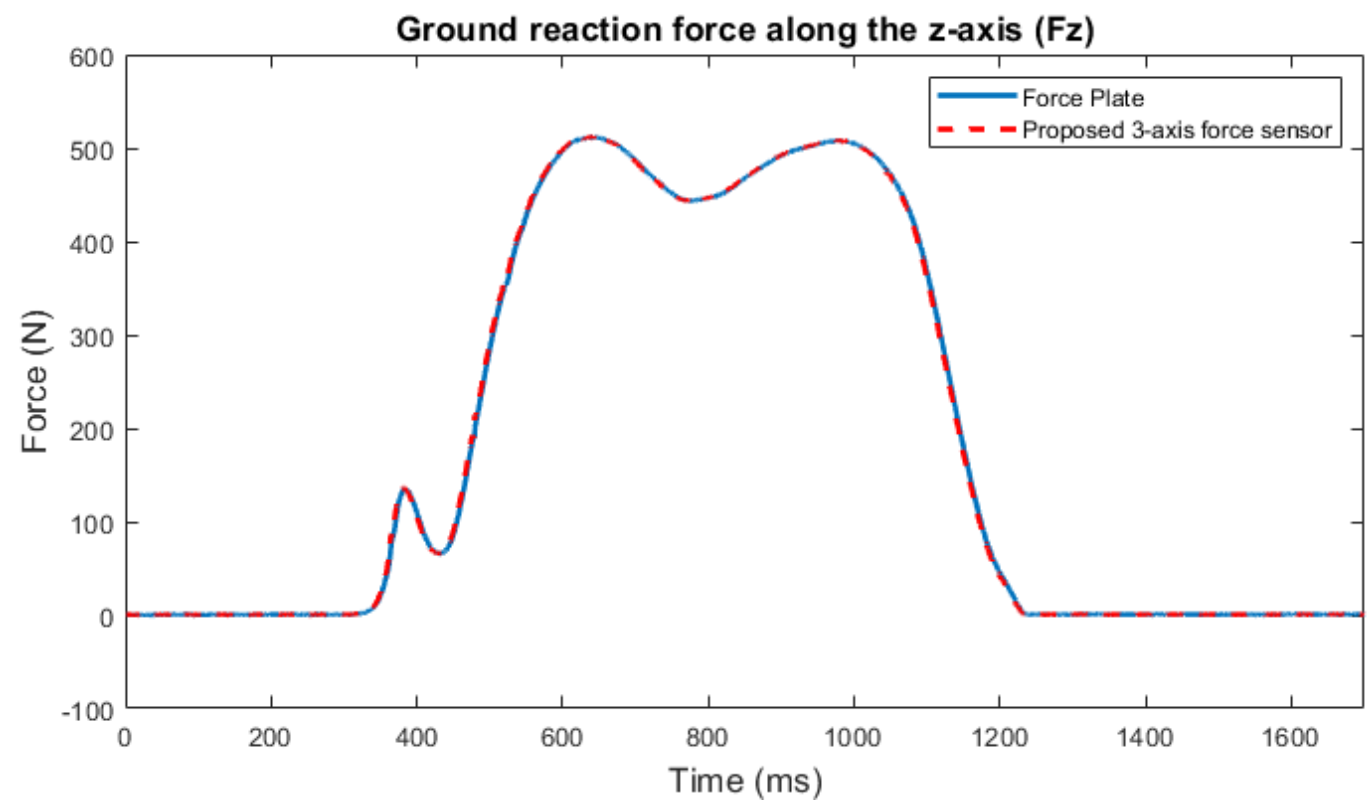

Figure 1.18: One step test of ground reaction force along the z-direction in comparison to the force plate. measurements 


\section{A.2 Specification of the compliant Force Sensor}

In order to determine the sensitivities of the electric sensing element (light-to-voltage convertor LTV), silicon rubber blocks and the sensor as whole integrated force measurement system, several experiments were carried out using a Servo-hydraulic Testing Systems (MTS) to measure the output-voltage and displacement (rubber deflection) as a result of the supplied force. Figure 1.19 shows the silicon rubber (compliant blocks) response to the force applied using the MTS, illustrating the relationship between the applied force and the generated deformation. It is known for any flexible martial to exhibit a nonlinear behaviour. However, the silicon rubber used shows a slightly force-deflection nonlinear relationship due the small deflection range (0 up to $2.5 \mathrm{~mm}$ ) compared to the amount of the applied force as shown Figure 1.19.

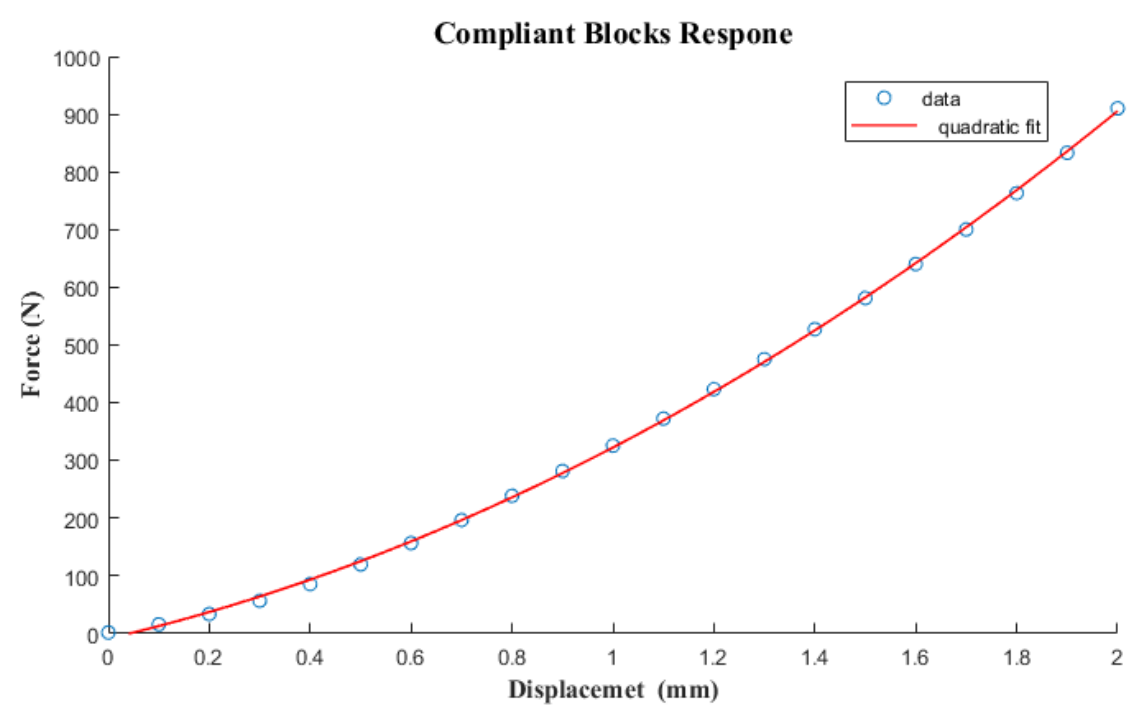

Figure 1.19: The experimental results of the rubber block due to the applied force. The material exhibits slightly nonlinear force-deflection relationship.

To determine the silicon rubber sensitivity, force-deflection relationship is assumed to be linear since it is almost linear. Compliant blocks sensitivity is the ratio of the 
incremental variation of the mechanical deformation (output) $\Delta d$ to the incremental variation of the input force $\Delta F$ which is given by:

$$
S_{\text {rubber }}=\frac{\Delta d}{\Delta F}=0.0018 \mathrm{~mm} / \mathrm{N} \text { or } 18 \mu \mathrm{m} / \mathrm{N}
$$

During the application of the force the output voltage (light intensity) of the optical sensing element (LTV) is also measured. As shown in Figure 3, light intensity to displacement relationship is also slightly nonlinear. The rang of the applied displacement in this test is between $0 \mathrm{~mm}$ to $2.1 \mathrm{~mm}$. In the same previous way, the optical sensitivity is calculated as follows:

$$
S_{L T V}=\frac{\Delta v}{\Delta d}=0.17 \mathrm{~V} / \mathrm{mm}
$$

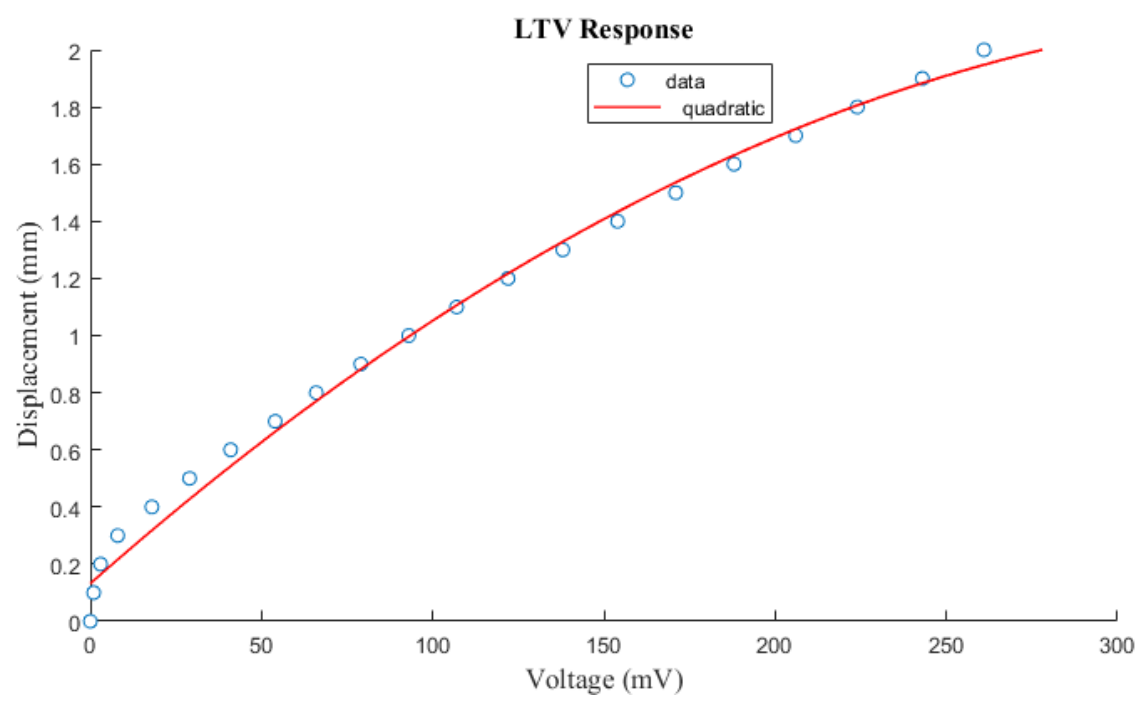

Figure 1.20: The experimental results of the light-to-voltage converter due to the displacements generated as a result of the applied force which also exhibits a slightly a nonlinear behaviour.

The overall sensitivity of the proposed sensor as whole integrated measurement system is the combination of both previous curves of light intensity-displacement 
relationship and force-deflection relationship which results in a linear final sensor response (the final output of the sensor ) exhibiting a linear force to voltage correlation. This proves the previous discussion of the nonlinearity cancellation phenomenon seen in the sensor response as shown in Figure 1.21. The sensor sensitivity is also calculated using the same previous formula which was $0.29 \mathrm{mV} / \mathrm{N}$.

The resolution of the developed sensor is the smallest variation in mechanical deformation input (the applied force) that can generate a change in the output voltage signal which was found to be $0.11131 \mathrm{~N}$.

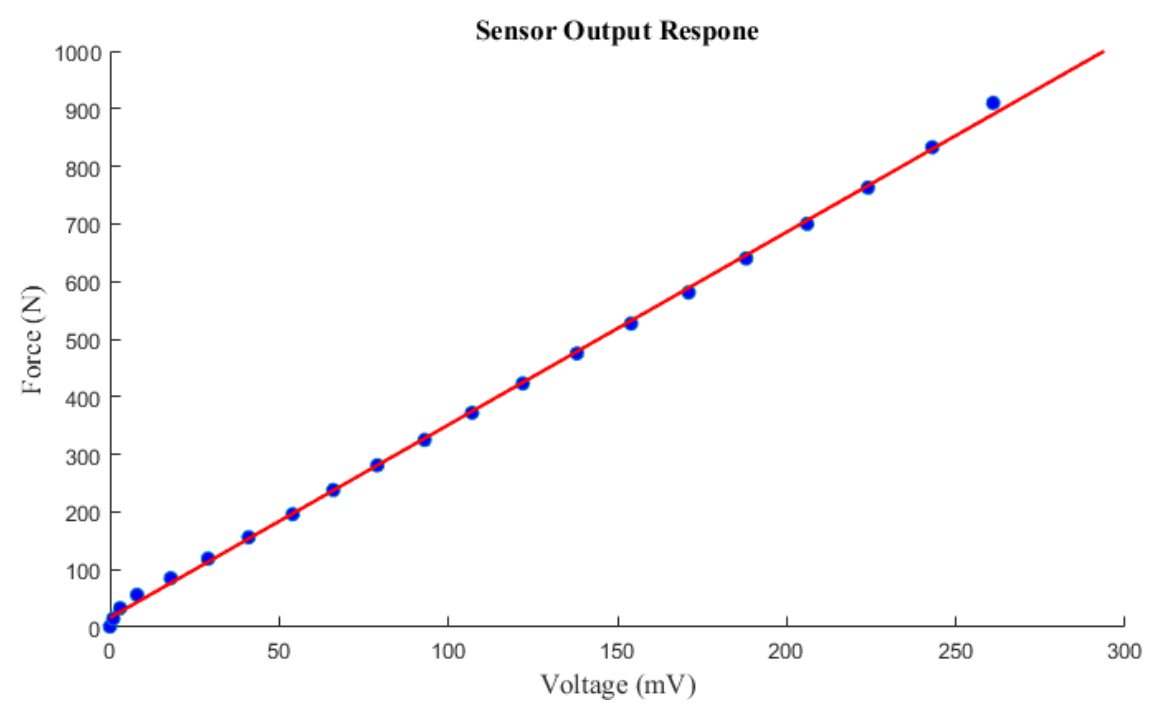

Figure 1.21: The final sensor response under the applied force. The sensor shows good linear correlation between force and generated output voltage. 


\section{Appendix B}

\section{Experimental Results of the}

\section{intensity-based, 6-Axis F/T Sensor}

\section{B.1 Experimental Validation Through An Actual Gait Measurement Scenario}

In order to validate the developed 6-axis force and torque sensor and its capability to measure the total ground reaction force and moment, the sensor was tested while mounted to the ground as depicted in Figure 2.1. The sensor was affixed to a standard force plate from Bertec Corporation. Gait data was recorded while a subject walked across the force plate and stepping on the sensor. Data was acquired as at a sampling rate of $1 \mathrm{kHz}$ using NI PCI 6229 DAQ from National Instrument. Sensor data was compared with a force and torque measurements obtained from the force plate.

Figure 2.3 illustrates a comparison between the performance of the developed 6-axis 


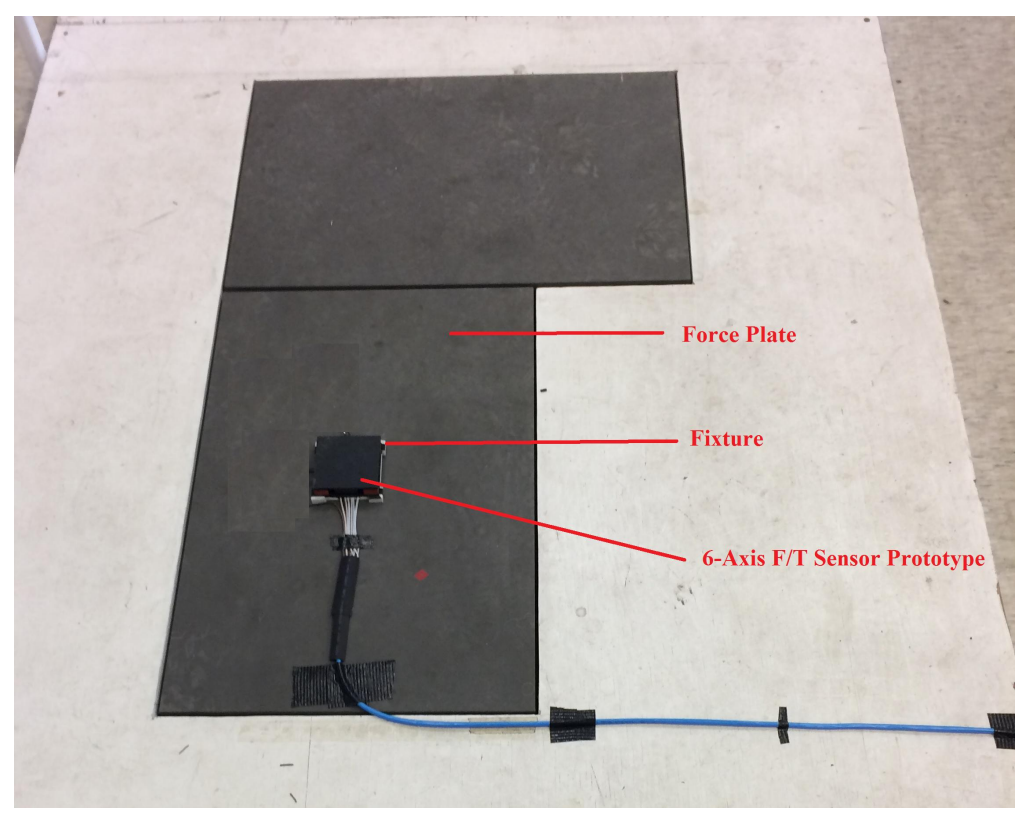

Figure 2.1: Real-time actual gait measurement scenario. The experimental setup is composed of the proposed light intensity-based,6-axis fiber optic, force and torque sensor and a standard force plate. the sensor is affixed on the force plate and the subject walks across the it.

$\mathrm{F} / \mathrm{T}$ sensor and results achieved from a standard force plate for each axis. The three plots seen in Figure 2.4 show the complete ground reaction force and moments in real-time for three subjects with different weight of $93 \mathrm{~kg}, 85 \mathrm{~kg}$ and $50 \mathrm{~kg}$ in sub-figures (a), (b) and (c) respectively. As it can be seen, the estimated F/T data closely match the measured data, indicating a reliable and high-quality performance of force and moment sensing. 


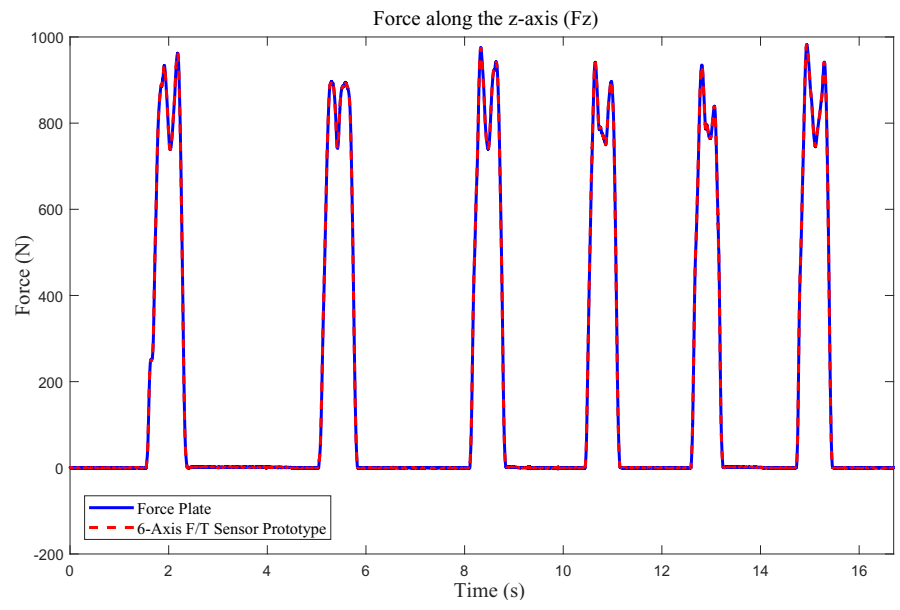

(a)

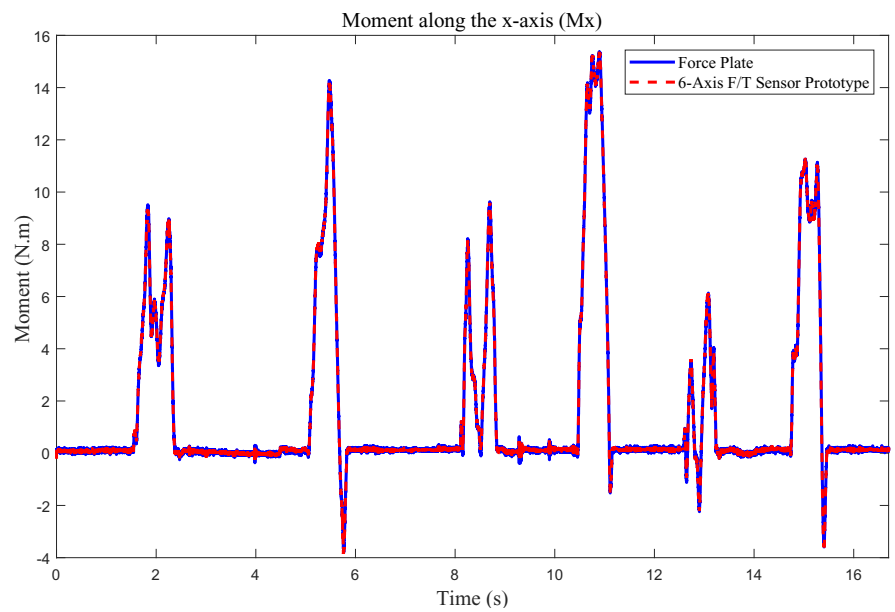

(b)

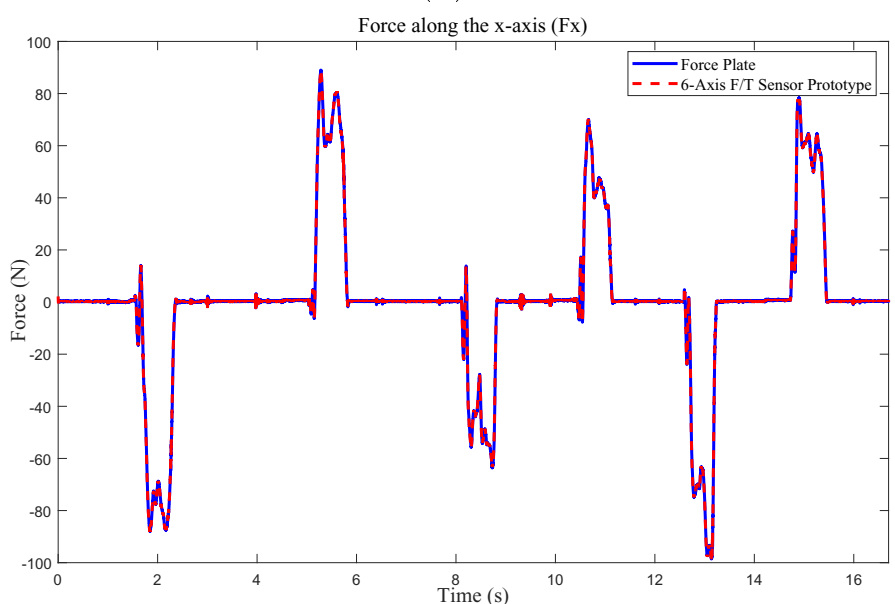

(c)

Figure 2.2: Experimental results through a real gait measurement for the applied forces. 


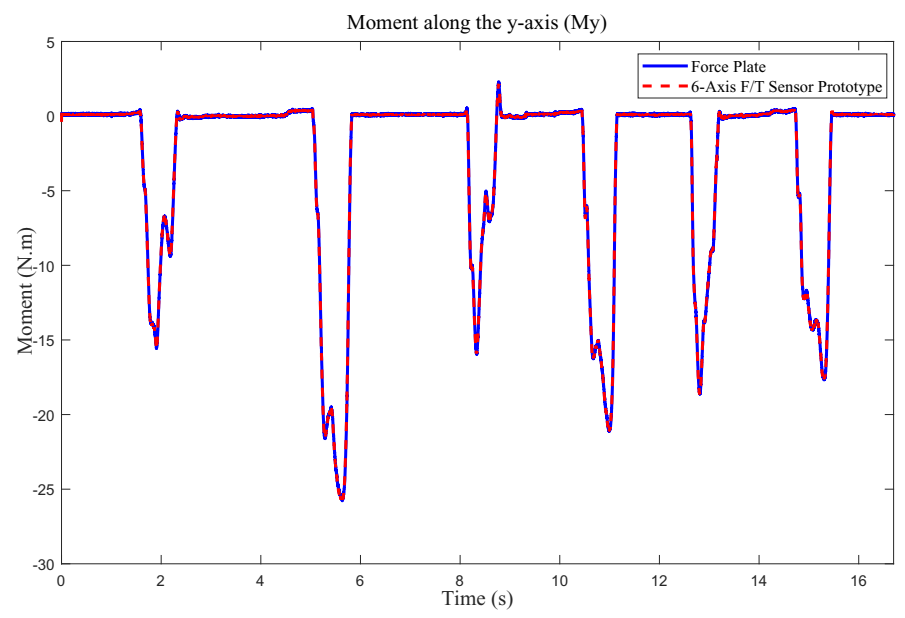

(a)

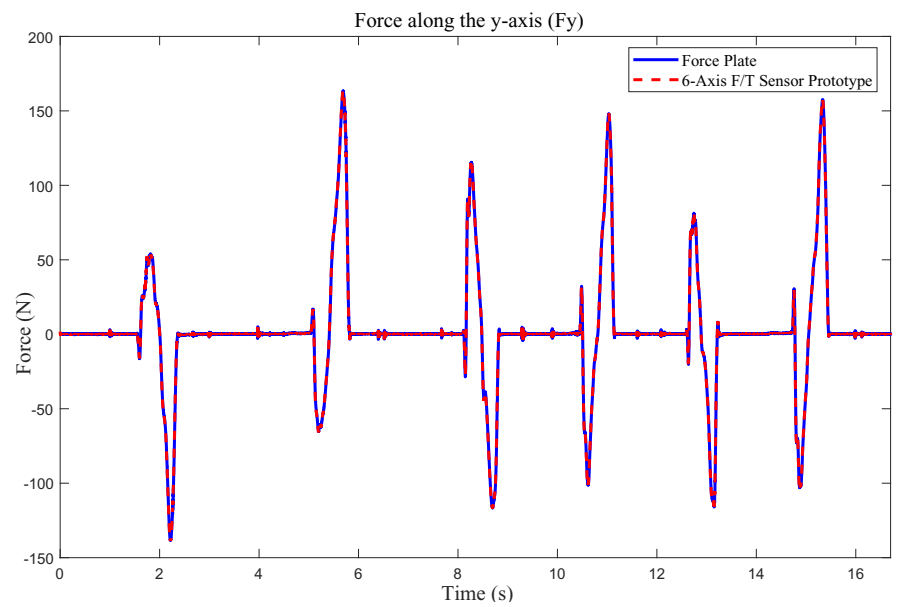

(b)

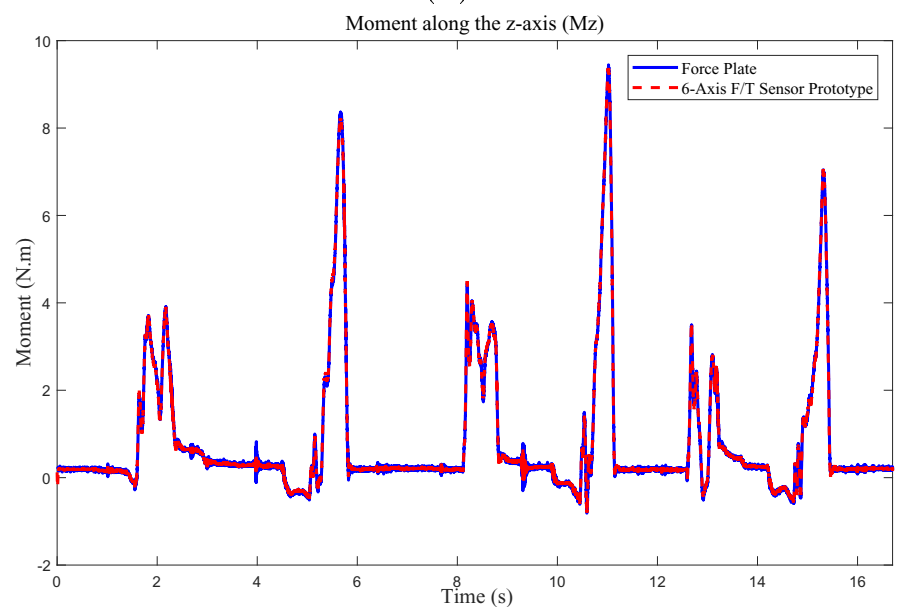

(c)

Figure 2.3: Experimental results through a real gait measurement for the applied moments. 


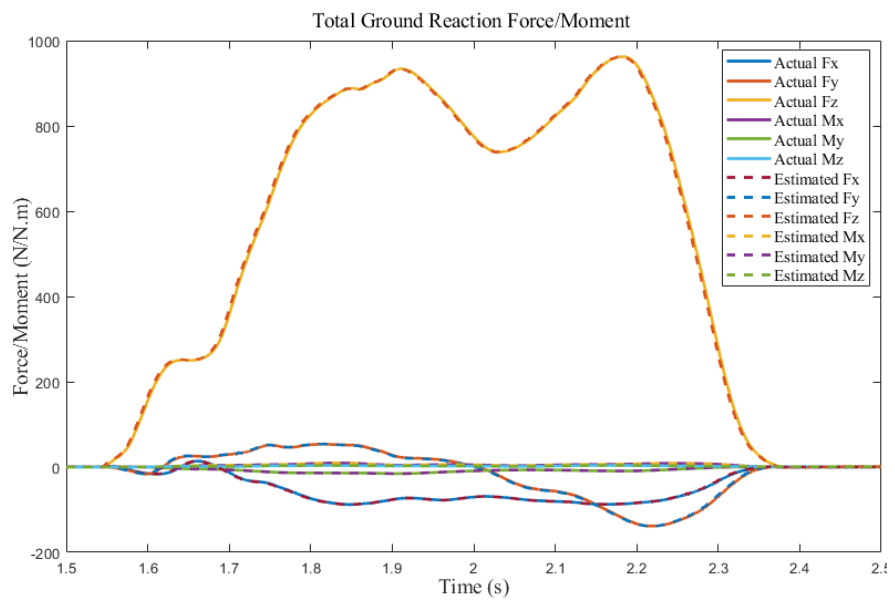

(a)

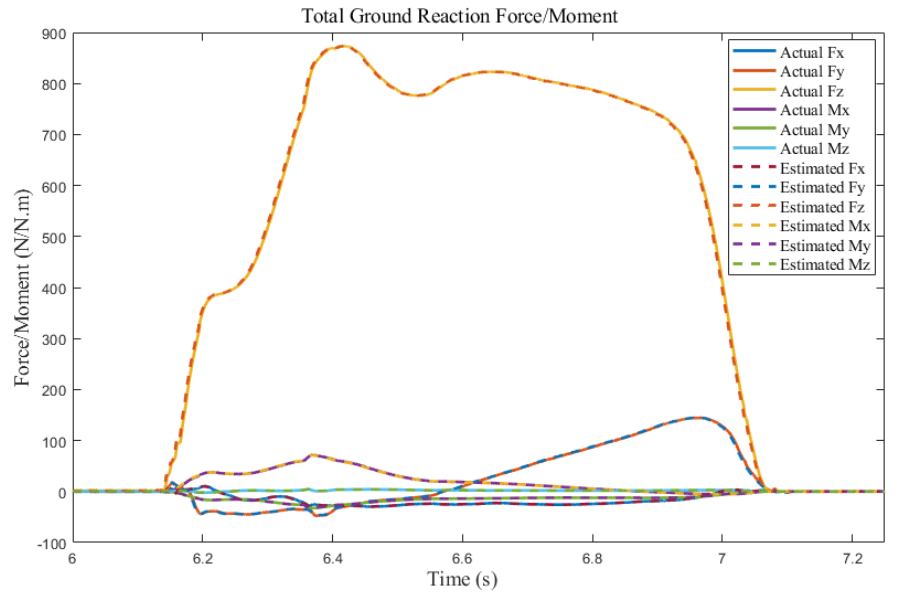

(b)

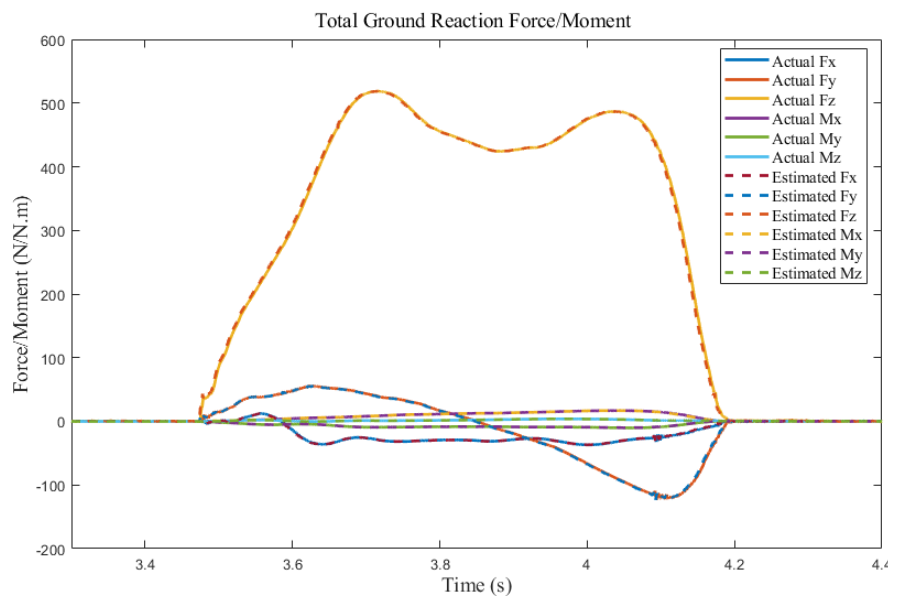

(c)

Figure 2.4: Experimental results through a real gait measurement . The dashed red line shows the estimated values of $\mathrm{F} / \mathrm{M}$ and using the proposed sensor an the blue line depicts the actual $\mathrm{F} / \mathrm{M}$ measured by the force plate. 


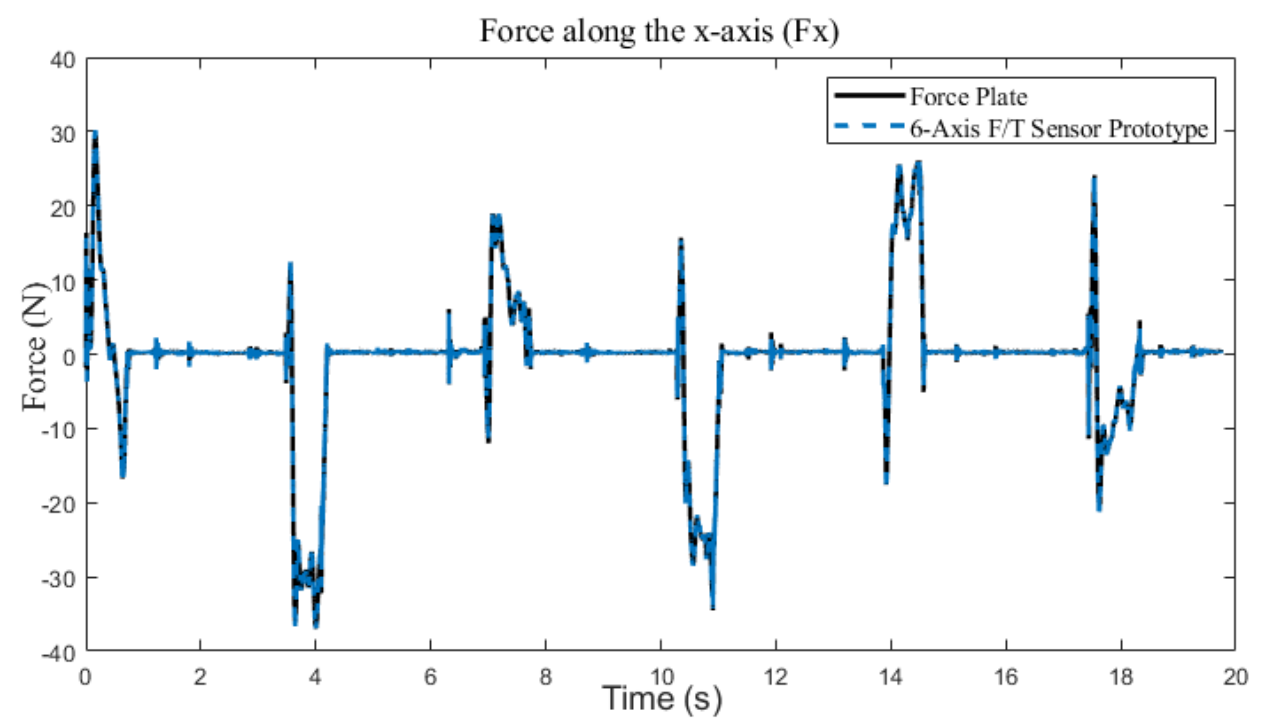

Figure 2.5: Experimental results of the proposed 6-axis force sensor during gait test of six steps while the shear force is applied along the $\mathrm{x}$-axis

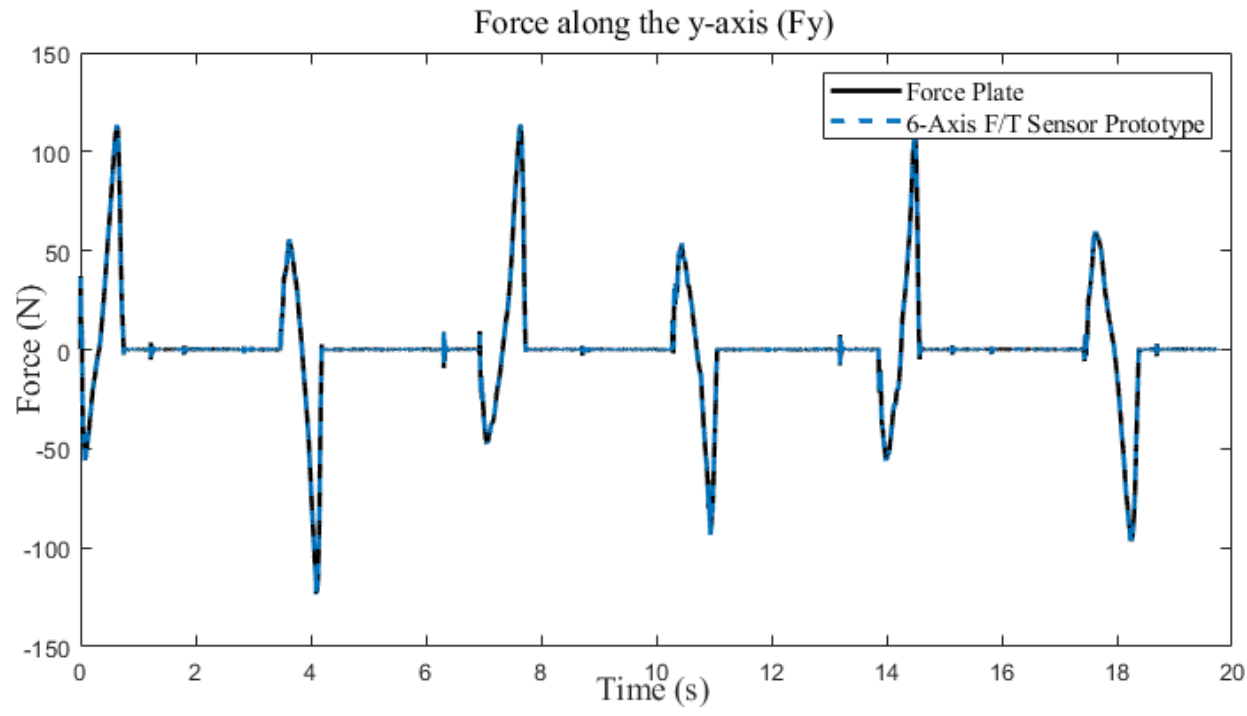

Figure 2.6: Ground reaction shear force acting along the y-axis showing six steps of the sensor performance compared to the force plate. 


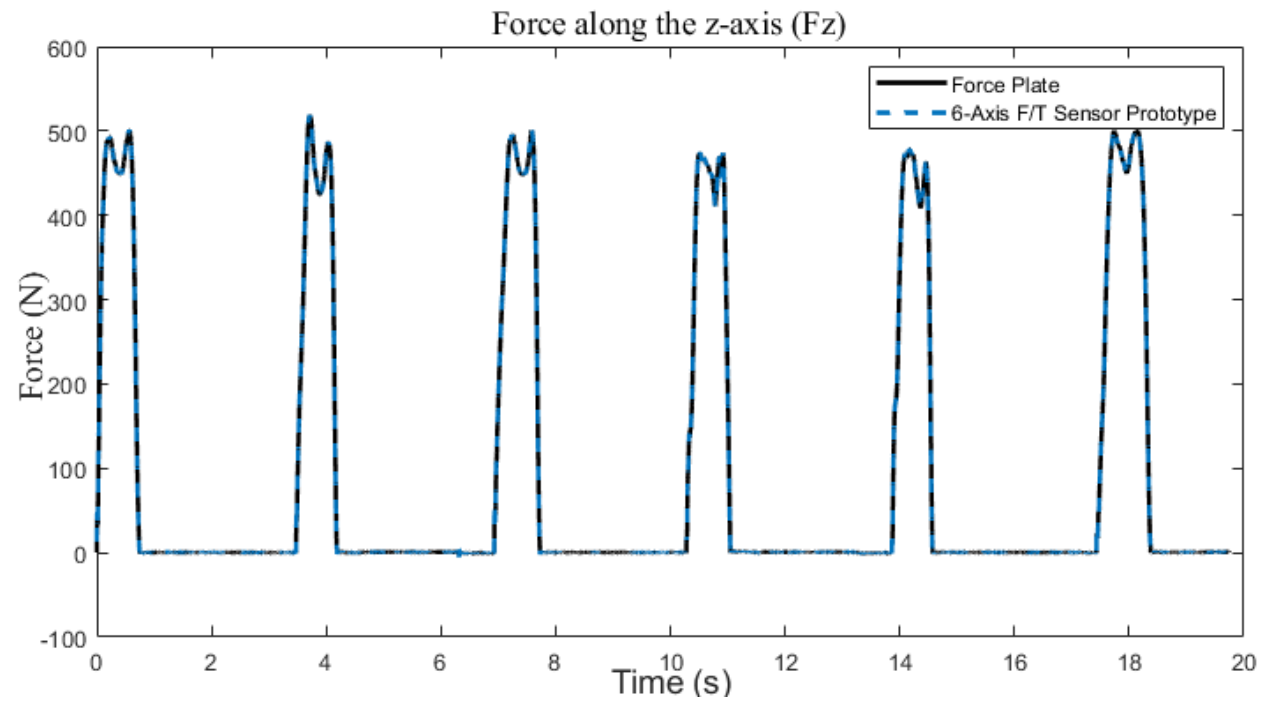

Figure 2.7: Experimental results of the proposed 6-axis force sensor during gait test of six steps while the normal force is applied along the $\mathrm{z}$-axis

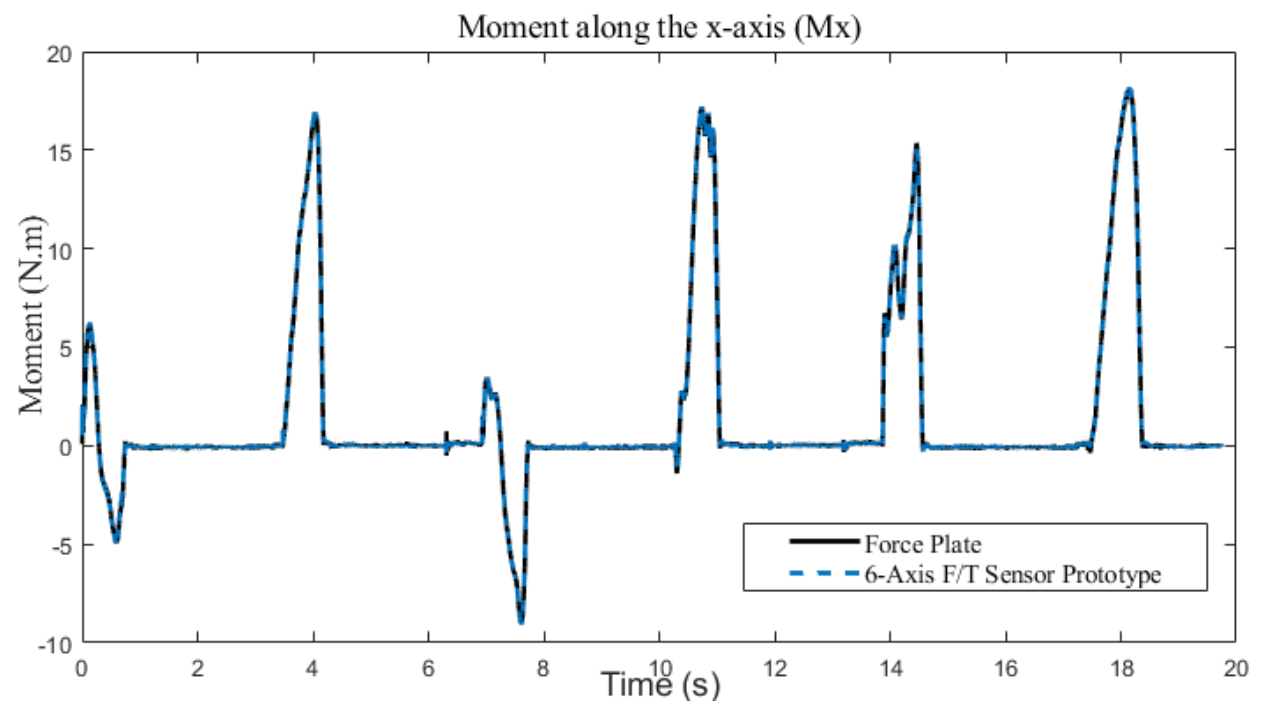

Figure 2.8: Ground reaction moment acting about the $\mathrm{x}$-axis showing six steps of the sensor performance compared to the force plate. 


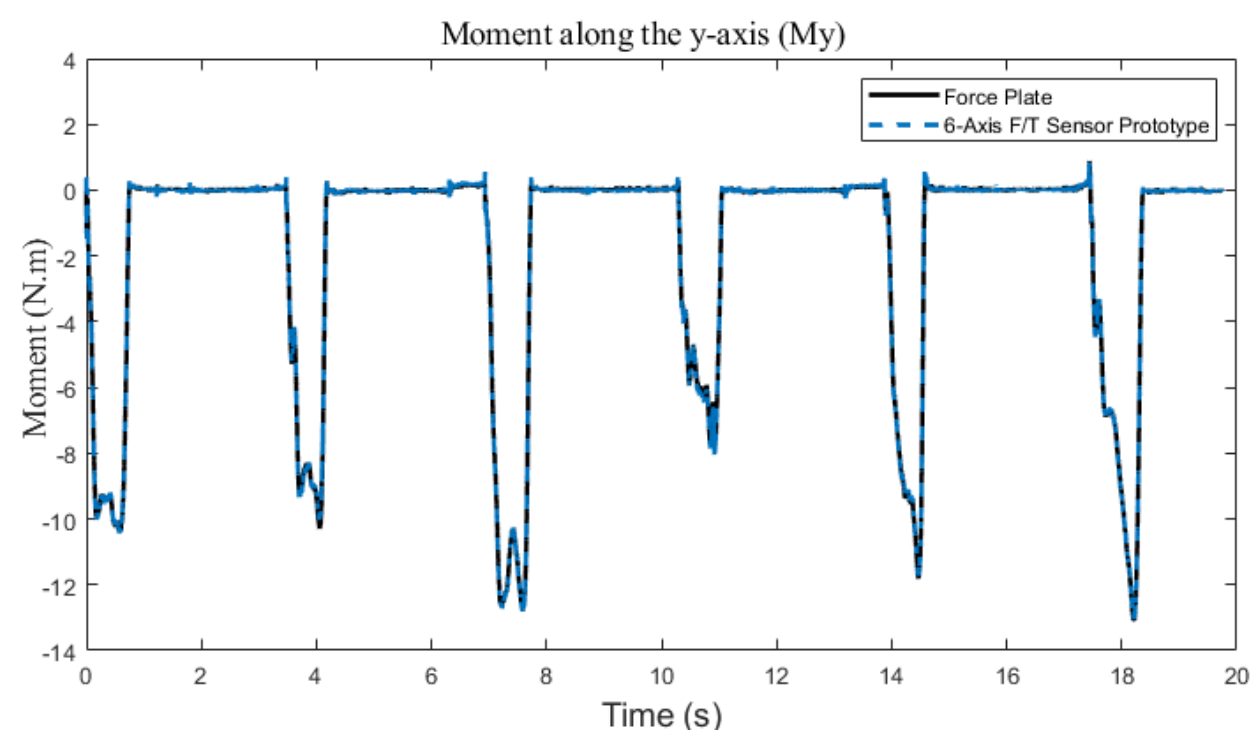

Figure 2.9: Ground reaction moment acting about the y-axis showing six steps of the sensor performance compared to the force plate.

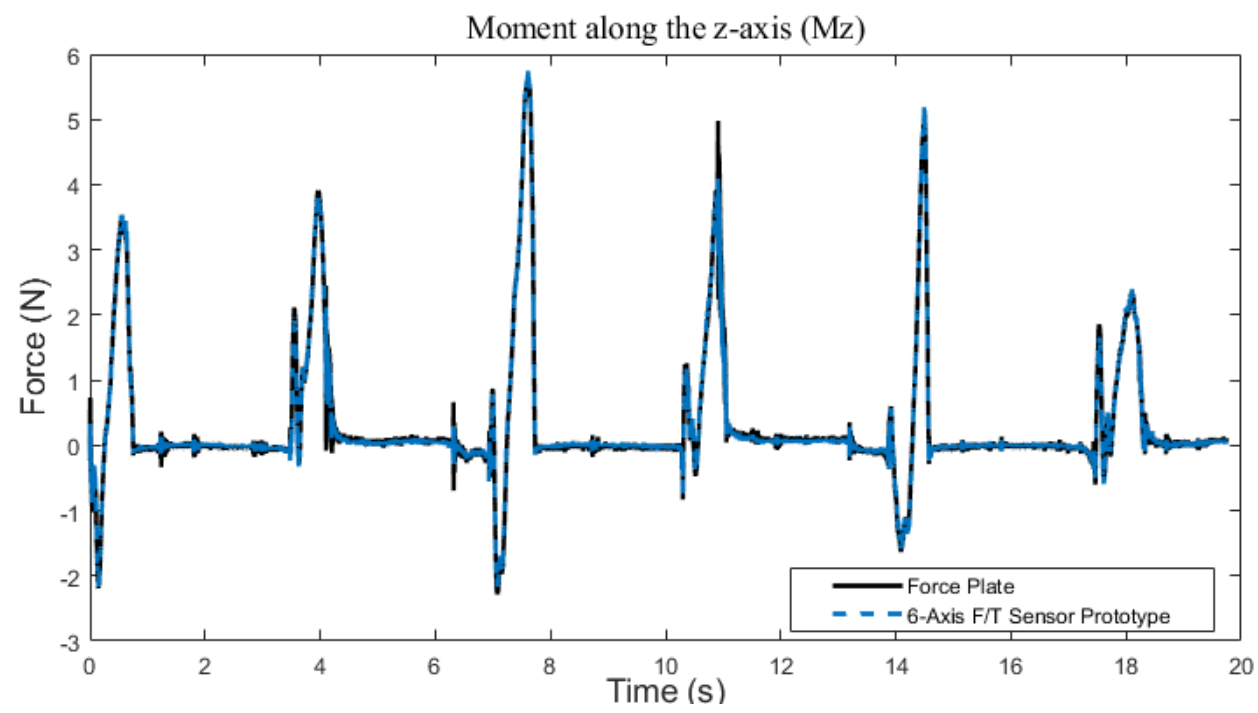

Figure 2.10: Ground reaction moment acting about the z-axis showing six steps of the sensor performance compared to the force plate. 


\section{B.2 Frequency Response Function}

Frequency response measurements are extensively used in modal analysis of different electrical and mechanical systems. Frequency response function (FRF) is essentially complex measurements and contains both imaginary and real components that can be analysed into phase and magnitude. Modal analysis is being widely used since the last three decades in several engineering technologies and different fields for solving, improving and optimizing the dynamic behaviour and the characteristics of various engineering systems [324, 325]. The transient response and the Bode plot of the second order state space model in comparison to the transient response of the third order state space model are shown below:

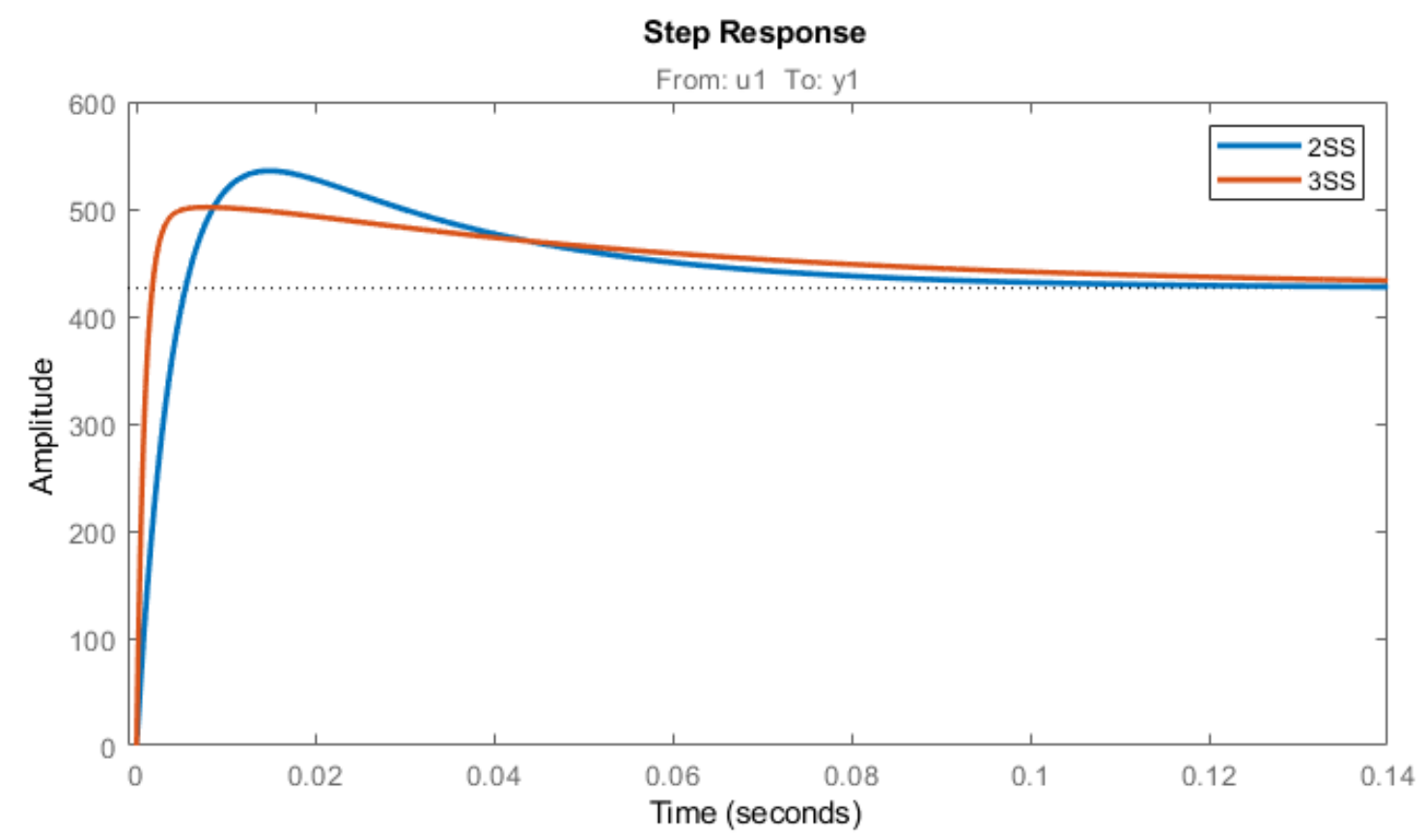

Figure 2.11: The transient response of the proposed second order compared to the third-order estimation models

The proposed dynamic estimation models exhibit excellent results and satisfactory 

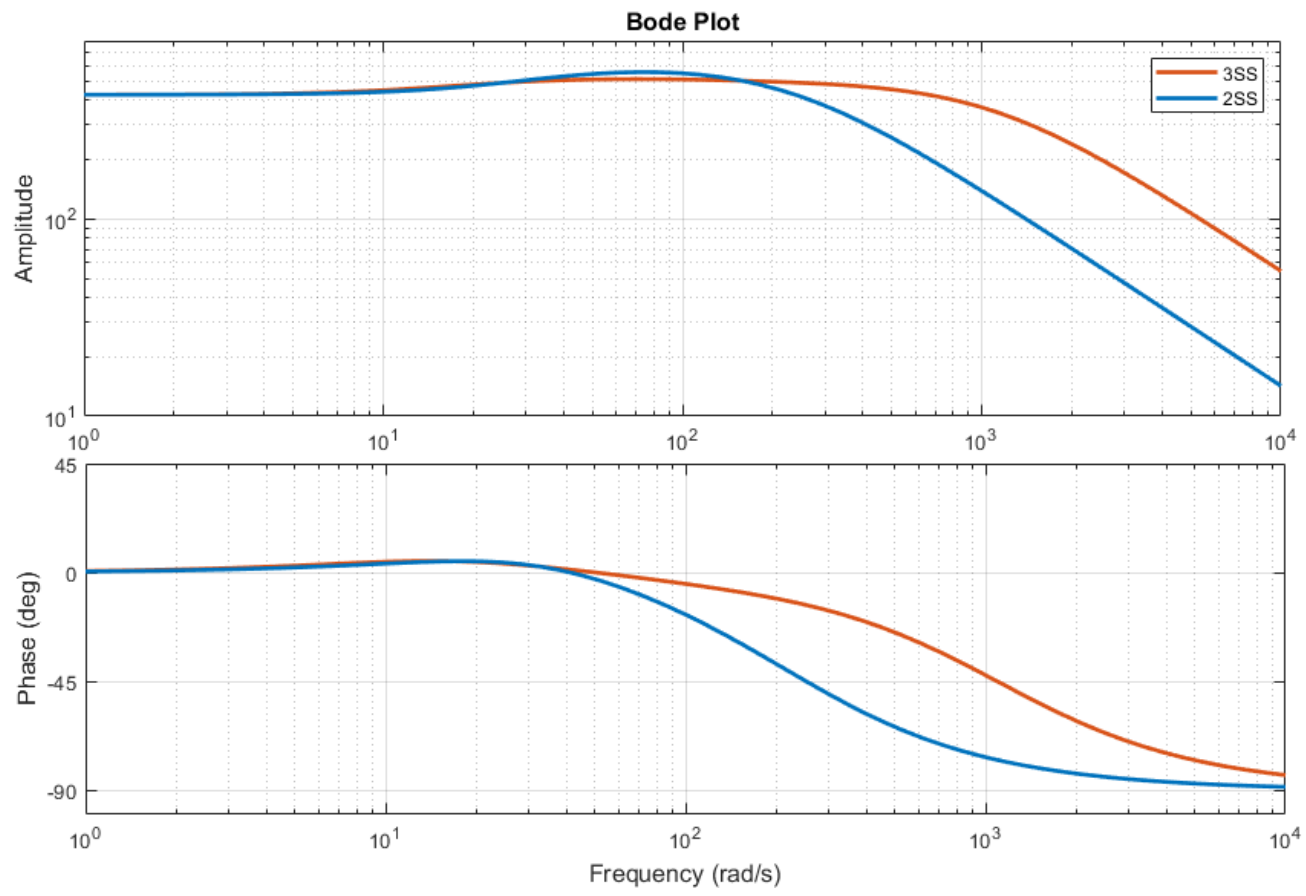

Figure 2.12: The bode plot of the proposed second order and the third-order state space estimation models that shows a significant improvements in bandwidth of compliant force and torque sensor.

bandwidth. The compliance of the sensor decreases the bandwidth. However, since the stiffness of the compliant material (rubber) used can be adjusted by changing the mixing ratio of the curing agent and the rubber, the bandwidth of the sensor can be controlled by increasing the stiffness of the rubber, and thus, the spring constant $(\mathrm{k})$ of the sensor system can be increased. This is a function of application and it can be adapted by using different materials with different stiffness. The spring constant of the proposed sensor was also calculated using Hooke's law which was found to be 455 $\mathrm{kN} / \mathrm{m}$.

The high-quality performance of the compliant sensor stems from the output voltage profile generated by the sensor due to the applied external forces, which has a very similar output voltage profile to the reference force/torque sensor. The resulting voltage of the proposed sensor obtained from the z-axis force sensing element (Fz) is shown 
in Figure 2.13 (b) when the sensor is continuously struck, generating a high frequency normal force ranging between $-5 \mathrm{~N}$ and $+25 \mathrm{~N}$. Figure 2.13 illustrates the force along the z-axis for both the reference sensor (a) using a strain gauge element and the proposed sensor (b), all as output voltages over time. This illustrates the difference between voltages as a change in magnitude and a shift in phase while the curvature of the lines exhibit the same voltage versus time profile. 


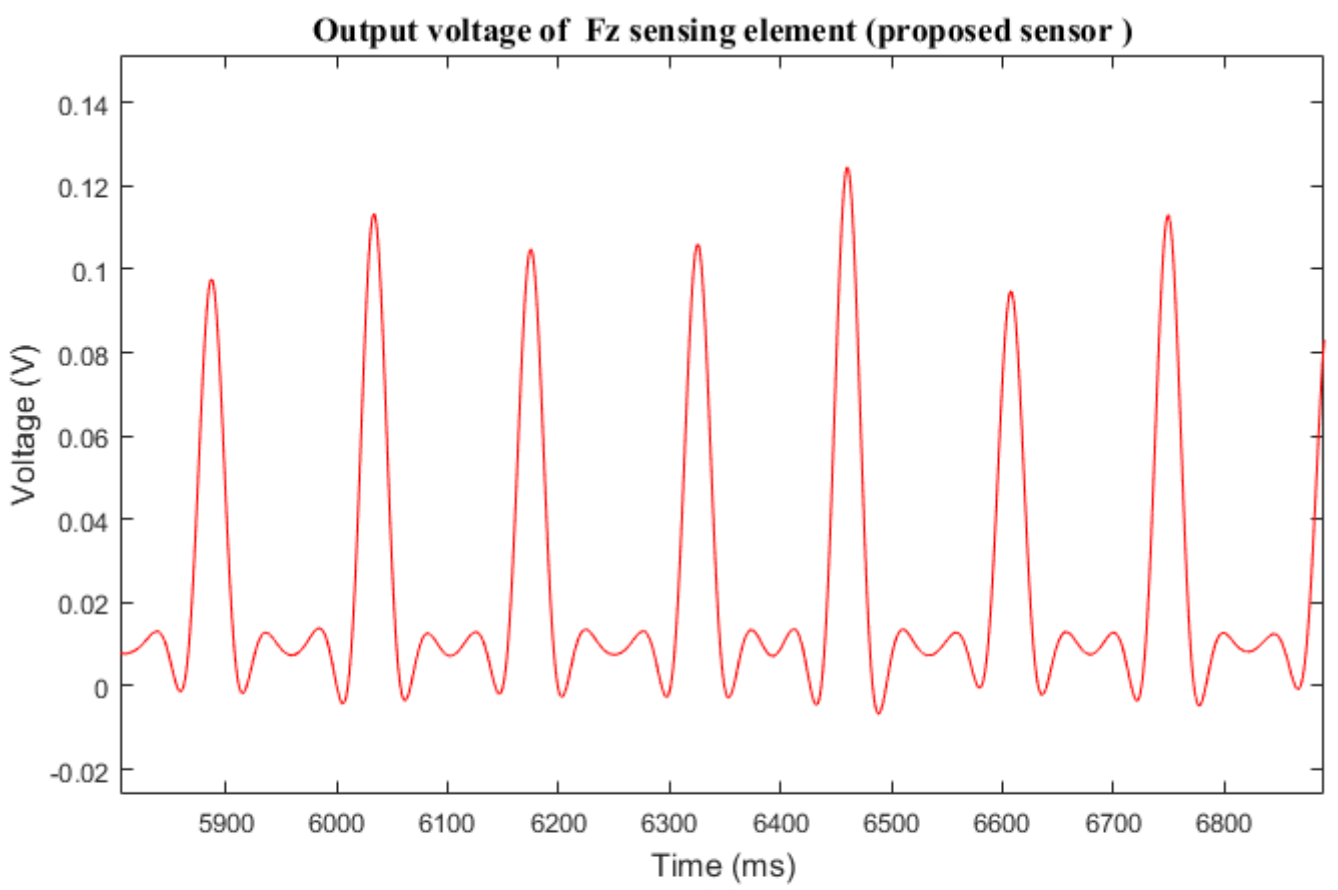

(a)

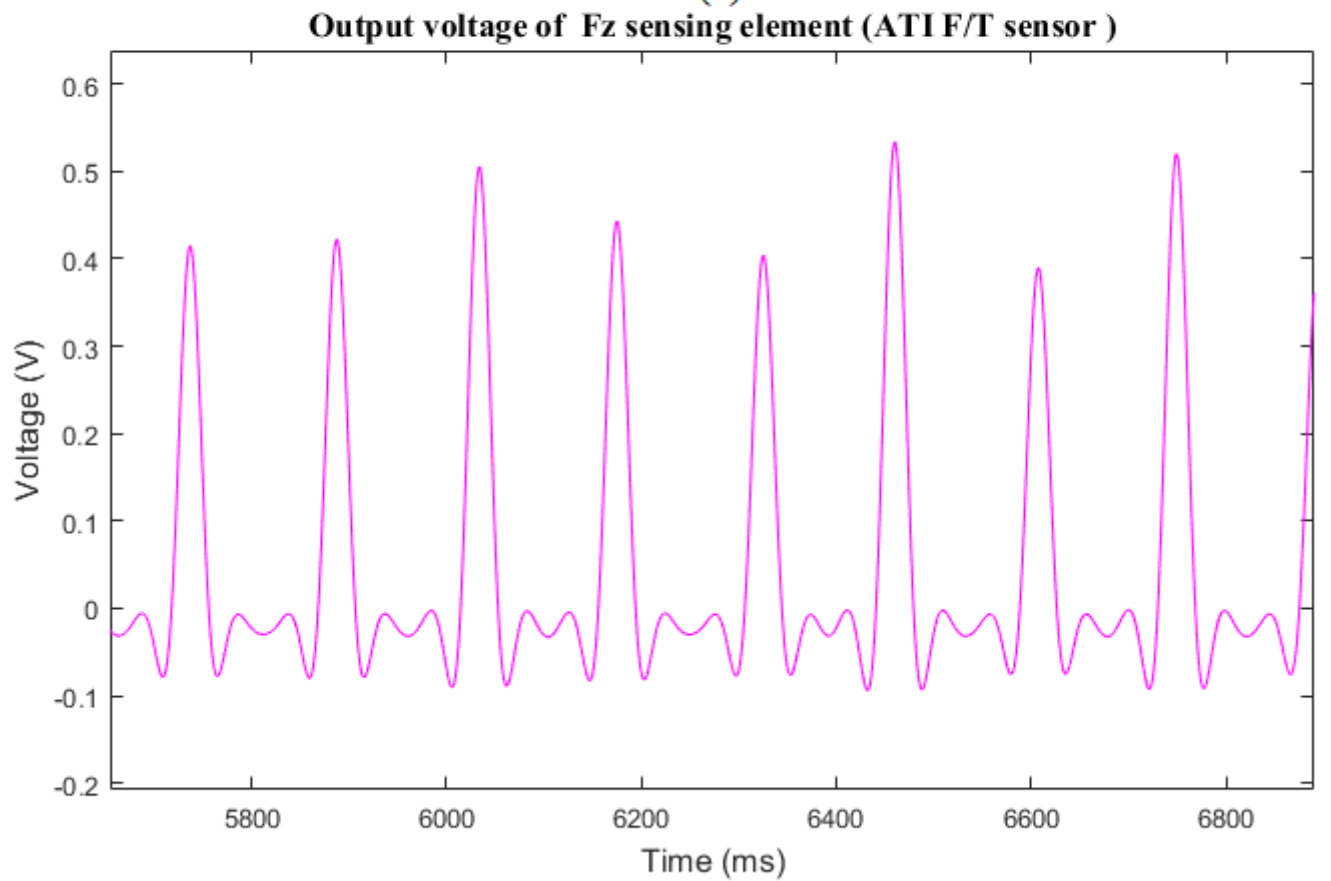

(b)

Figure 2.13: A comparison between the resulting output voltages. (a) shows the output voltage data recorded from the reference sensor. (b) demonstrates the resulting output voltage of the proposed sensor obtained from the optical sensing element. 


\section{Appendix C}

\section{FBG-Based Force Sensing Elements:}

\section{Experimental Results}

Data was recorded using an optical sensing analyzer, from Micron Optics, USA. Si720 is high accuracy, high resolution, high power FBG interrogation system that can be used in various applications of fiber optic sensors. The system has a built-in monitor which provides the user with full understanding of the fiber Bragg grating spectrum and shows how the reflected Bragg wavelength shifts when the physical condition changes. This optical device is used to detect the change in the reflected Brag wavelength of the

proposed sensor. Since the recording speed of the optical sensing analyzer is around $2.5 \mathrm{~Hz}$, the sampling rate of the ATI 6-Axis force and torque sensor is set to the same as the optical sensing analyzer. Figure 3.2 shows the si720 FBG integration system. Figure 3.2 shows the si720 FBG integration system. 


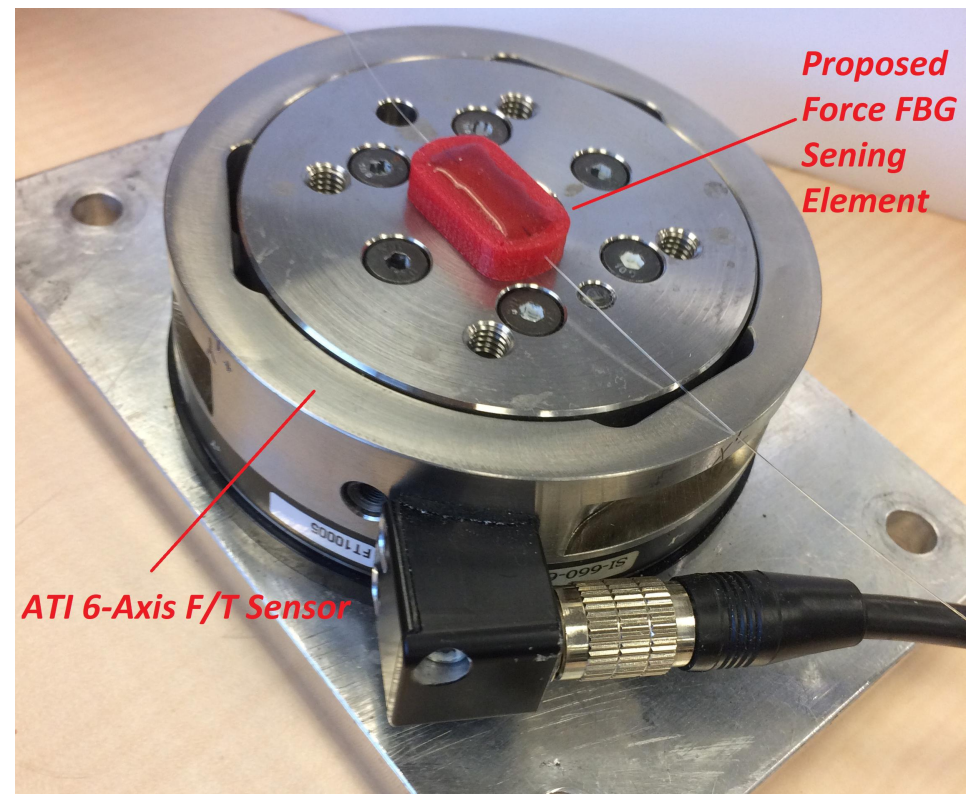

Figure 3.1: Experimental setup used for online force data recording. The developed sensor is attached to the ATI 6-axis F/T sensor reference from Industrial Automation. the proposed sensor is connected to si720 optical analyser and the refrence data acquired using Real-Time Simulink, MATLAB, for a comparison.

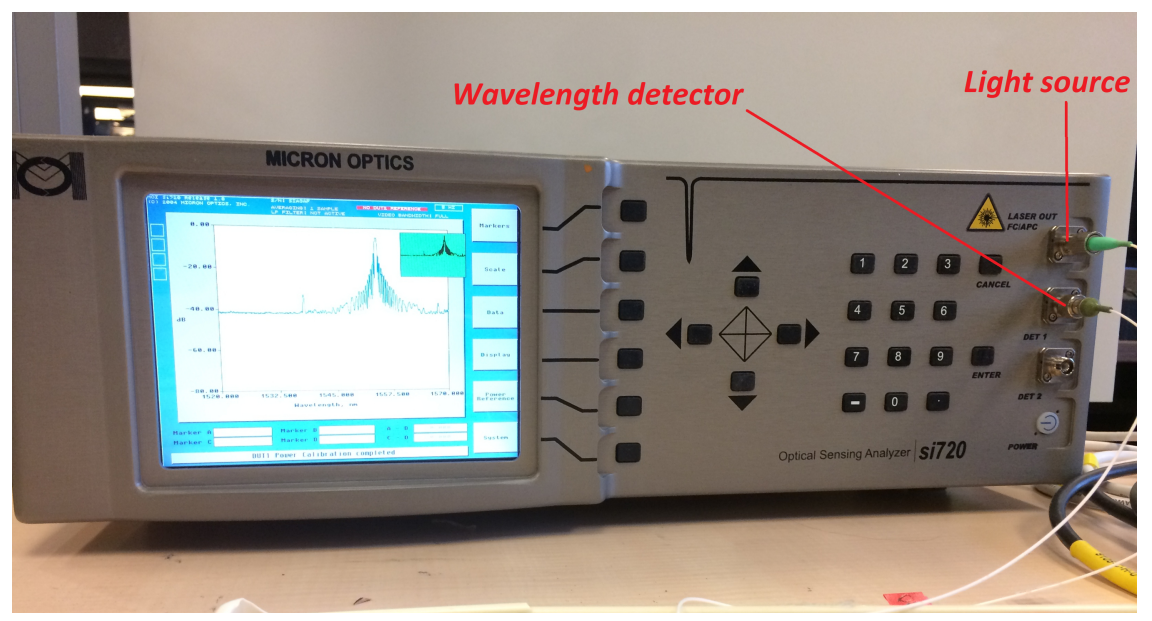

Figure 3.2: FBG integration system: optical sensing analyzer, Si720 Micron Optics. The system is used to detect the change reflected Bragg wavelength as a result of the applied force. 


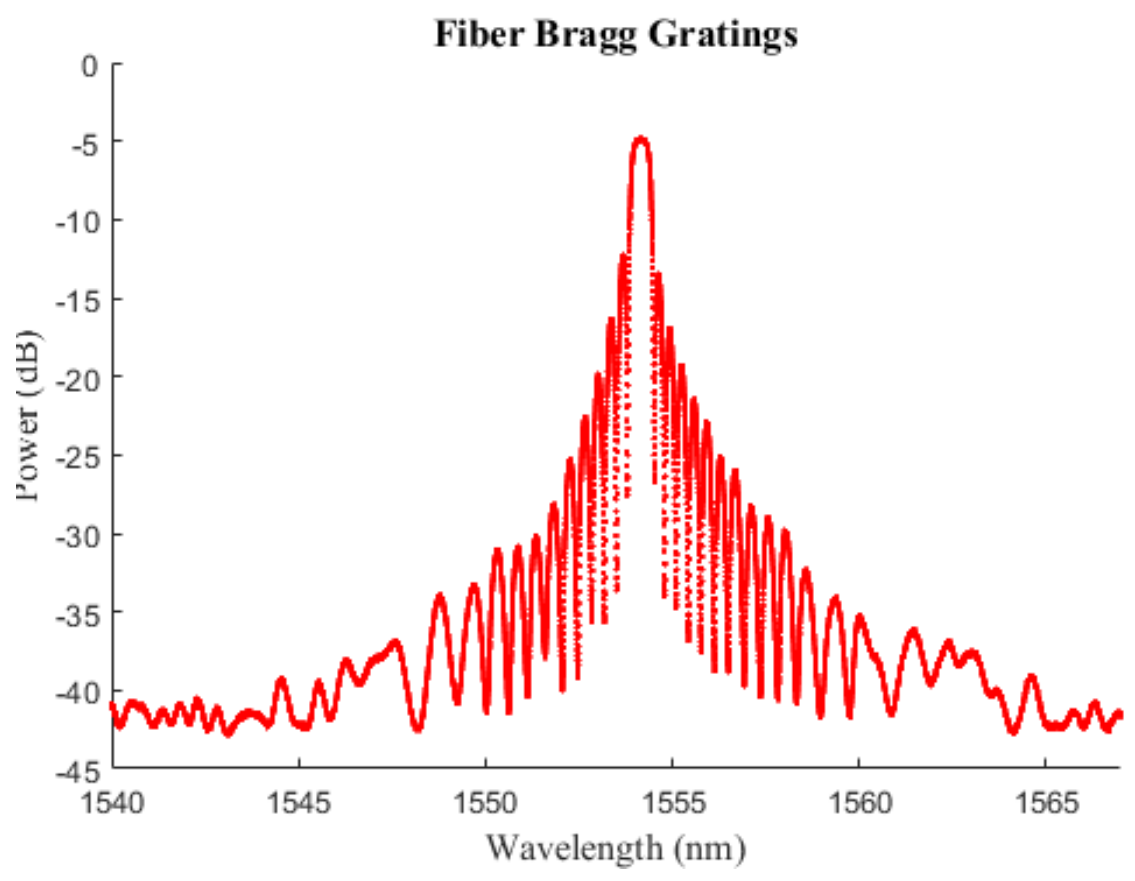

Figure 3.3: the measured reflected power versus the generated wavelength for the fabricated fiber Bragg grating along the optical fiber. This strong resonance is referred to as Bragg resonance which is created due this particular FBG.

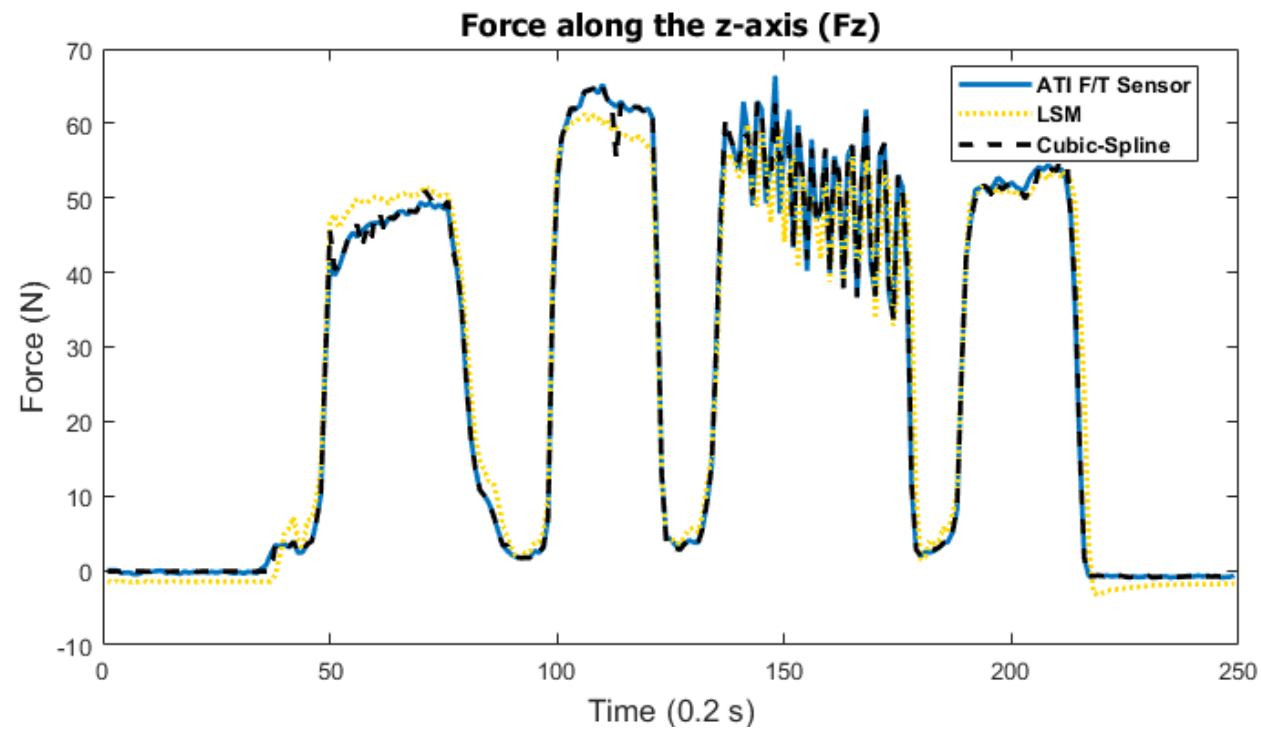

Figure 3.4: Normal force sensing element response due to the applied normal force along the $\mathrm{z}$-axis. A comparison between the performance of a simple linear least squares optimization and a spline function. 


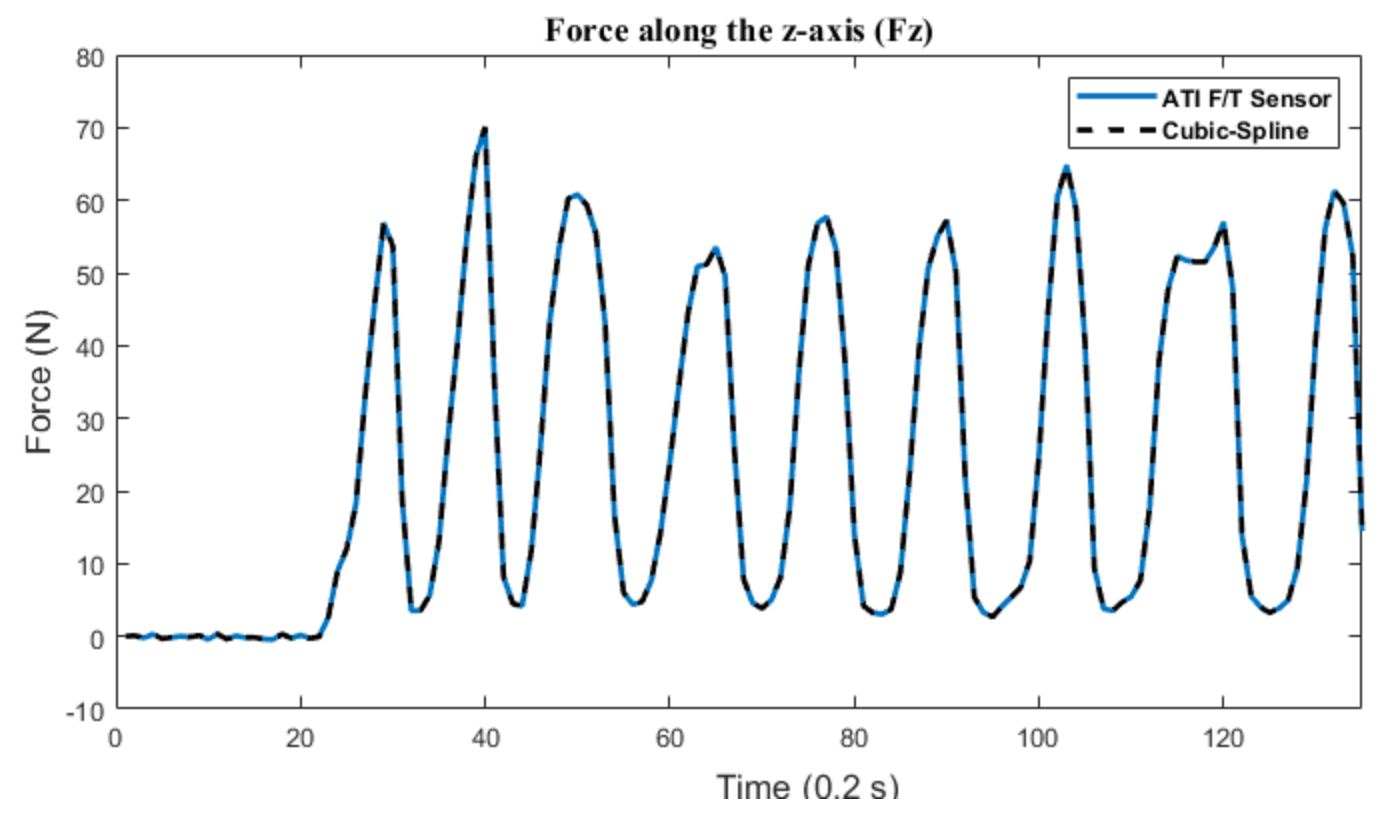

Figure 3.5: Normal force sensing element response due to the applied normal force along the z-axis using spline function as a calibration method

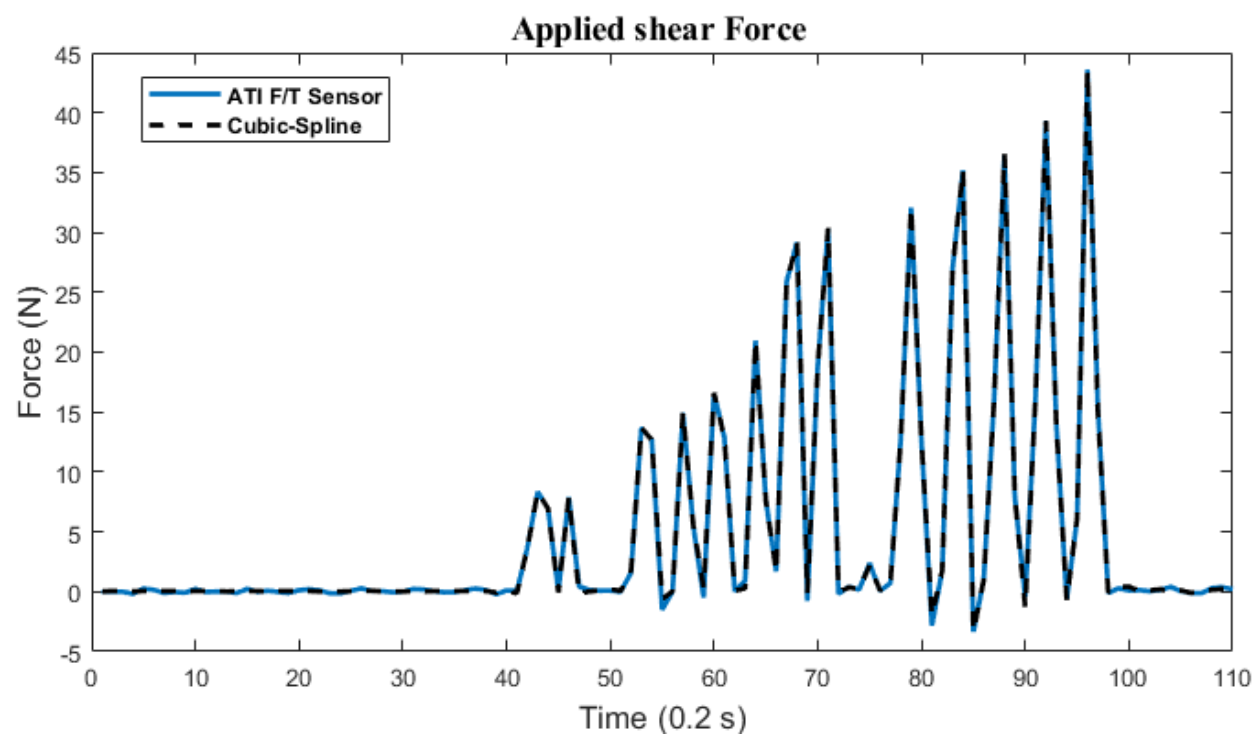

Figure 3.6: Shear force sensing element response due to the applied shear force. The performance of the sensing element when a spline function is used to characterize the sensor compared to the actual force. 


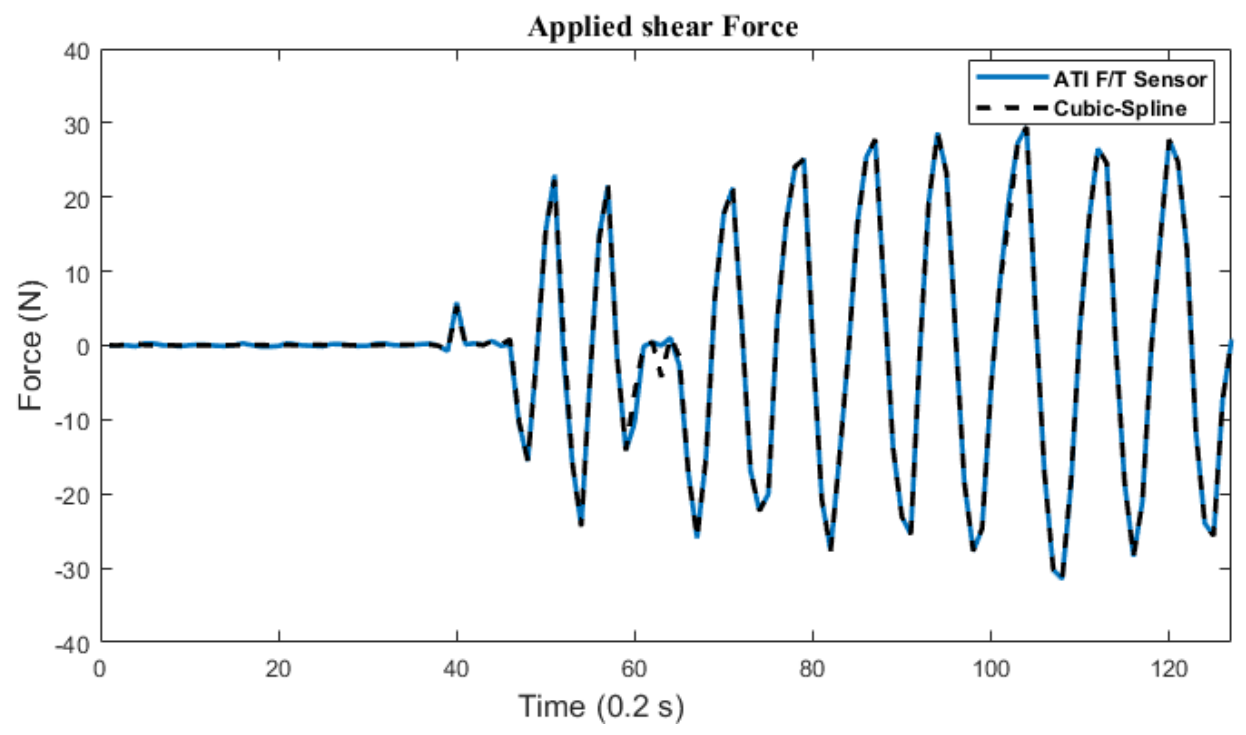

Figure 3.7: Shear force sensing element response due to the applied shear force compared to the actual force measured by ATI force and torque sensor. 


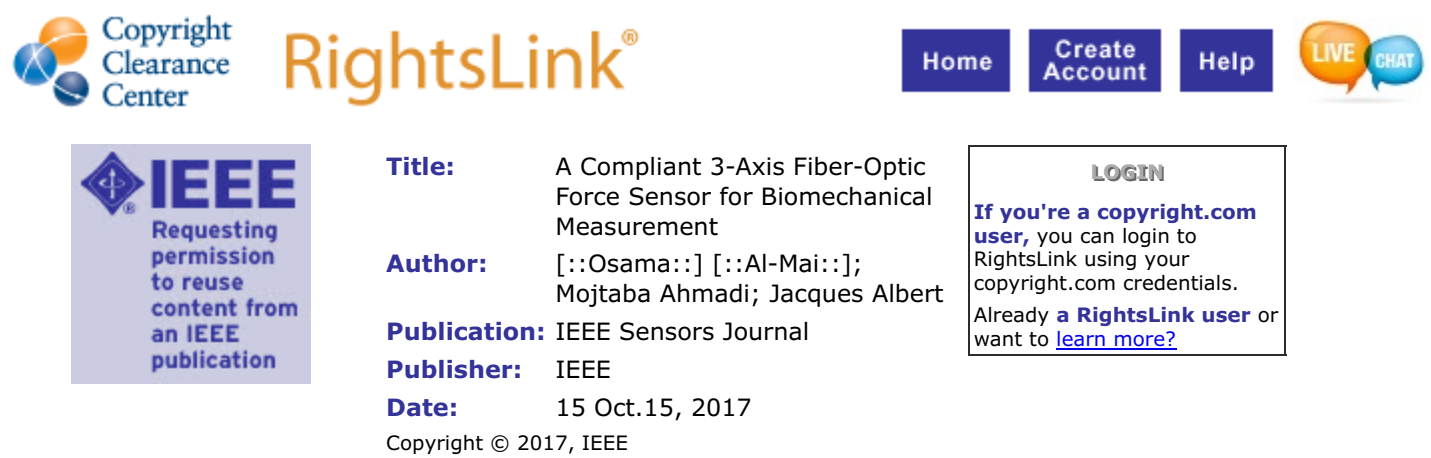

Thesis / Dissertation Reuse

The IEEE does not require individuals working on a thesis to obtain a formal reuse license, however, you may print out this statement to be used as a permission grant:

Requirements to be followed when using any portion (e.g., figure, graph, table, or textual material) of an IEEE copyrighted paper in a thesis:

1) In the case of textual material (e.g., using short quotes or referring to the work within these papers) users must give full credit to the original source (author, paper, publication) followed by the IEEE copyright line (c) 2011 IEEE.

2) In the case of illustrations or tabular material, we require that the copyright line $(\subset$ [Year of original publication] IEEE appear prominently with each reprinted figure and/or table.

3) If a substantial portion of the original paper is to be used, and if you are not the senior author, also obtain the senior author's approval.

Requirements to be followed when using an entire IEEE copyrighted paper in a thesis:

1) The following IEEE copyright/ credit notice should be placed prominently in the references: (C) [year of original publication] IEEE. Reprinted, with permission, from [author names, paper title, IEEE publication title, and month/year of publication]

2) Only the accepted version of an IEEE copyrighted paper can be used when posting the paper or your thesis on-line.

3) In placing the thesis on the author's university website, please display the following message in a prominent place on the website: In reference to IEEE copyrighted material which is used with permission in this thesis, the IEEE does not endorse any of [university/educational entity's name goes here]'s products or services. Internal or personal use of this material is permitted. If interested in reprinting/republishing IEEE copyrighted material for advertising or promotional purposes or for creating new collective works for resale or redistribution, please go to http://www.ieee.org/publications standards/publications/rights/rights link.html to learn how to obtain a License from RightsLink.

If applicable, University Microfilms and/or ProQuest Library, or the Archives of Canada may supply single copies of the dissertation.

\section{BACK}

\section{CLOSE WINDOW}

Copyright (c) 2019 Copyright Clearance Center, Inc. All Rights Reserved. Privacy statement. Terms and Conditions. Comments? We would like to hear from you. E-mail us at customercare@copyright.com 


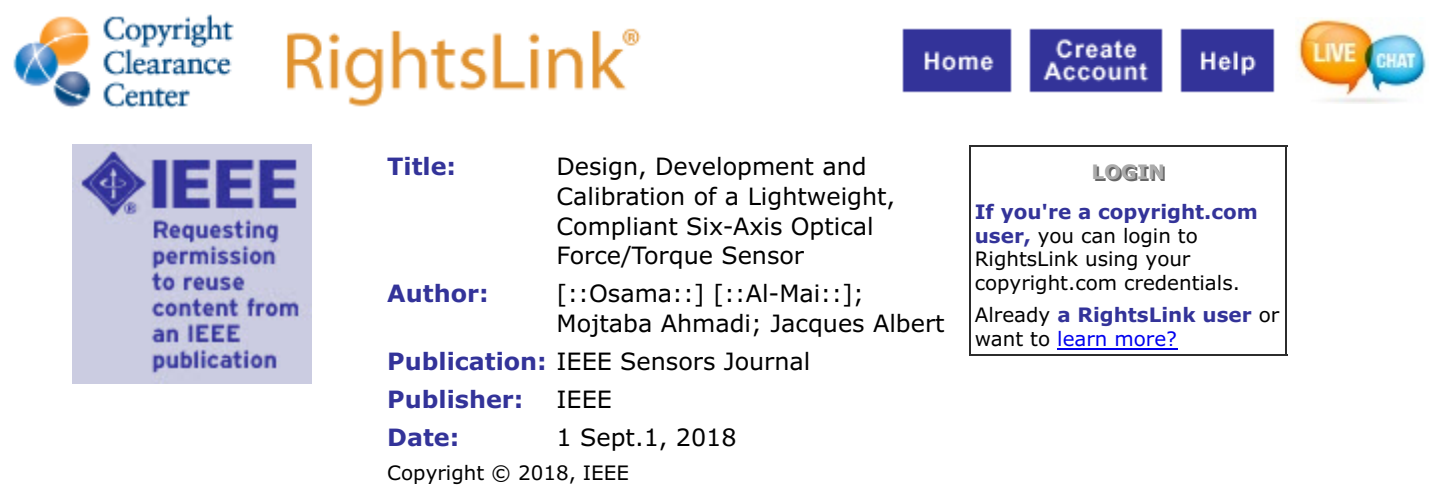

\section{Thesis / Dissertation Reuse}

The IEEE does not require individuals working on a thesis to obtain a formal reuse license, however, you may print out this statement to be used as a permission grant:

Requirements to be followed when using any portion (e.g., figure, graph, table, or textual material) of an IEEE copyrighted paper in a thesis:

1) In the case of textual material (e.g., using short quotes or referring to the work within these papers) users must give full credit to the original source (author, paper, publication) followed by the IEEE copyright line (c) 2011 IEEE.

2) In the case of illustrations or tabular material, we require that the copyright line $(\subset)$ [Year of original publication] IEEE appear prominently with each reprinted figure and/or table.

3) If a substantial portion of the original paper is to be used, and if you are not the senior author, also obtain the senior author's approval.

Requirements to be followed when using an entire IEEE copyrighted paper in a thesis:

1) The following IEEE copyright/ credit notice should be placed prominently in the references: (c) [year of original publication] IEEE. Reprinted, with permission, from [author names, paper title, IEEE publication title, and month/year of publication]

2) Only the accepted version of an IEEE copyrighted paper can be used when posting the paper or your thesis on-line.

3) In placing the thesis on the author's university website, please display the following message in a prominent place on the website: In reference to IEEE copyrighted material which is used with permission in this thesis, the IEEE does not endorse any of [university/educational entity's name goes here]'s products or services. Internal or personal use of this material is permitted. If interested in reprinting/republishing IEEE copyrighted material for advertising or promotional purposes or for creating new collective works for resale or redistribution, please go to http://www.ieee.org/publications standards/publications/rights/rights link.html to learn how to obtain a License from RightsLink.

If applicable, University Microfilms and/or ProQuest Library, or the Archives of Canada may supply single copies of the dissertation.

\section{BACK $\quad$ CLOSE WINDOW}

Copyright (c) 2019 Copyright Clearance Center, Inc. All Rights Reserved. Privacy statement. Terms and Conditions. Comments? We would like to hear from you. E-mail us at customercare@copyright.com 


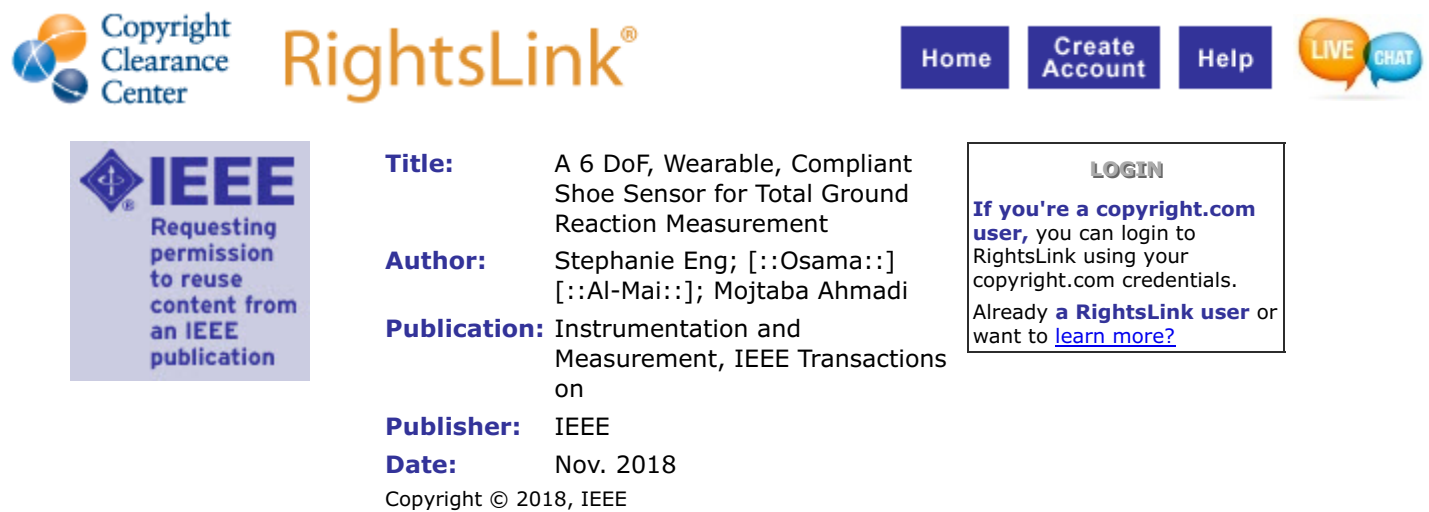

\section{Thesis / Dissertation Reuse}

The IEEE does not require individuals working on a thesis to obtain a formal reuse license, however, you may print out this statement to be used as a permission grant:

Requirements to be followed when using any portion (e.g., figure, graph, table, or textual material) of an IEEE copyrighted paper in a thesis:

1) In the case of textual material (e.g., using short quotes or referring to the work within these papers) users must give full credit to the original source (author, paper, publication) followed by the IEEE copyright line (c) 2011 IEEE.

2) In the case of illustrations or tabular material, we require that the copyright line (c) [Year of original publication] IEEE appear prominently with each reprinted figure and/or table.

3) If a substantial portion of the original paper is to be used, and if you are not the senior author, also obtain the senior author's approval.

Requirements to be followed when using an entire IEEE copyrighted paper in a thesis:

1) The following IEEE copyright/ credit notice should be placed prominently in the references: (c) [year of original publication] IEEE. Reprinted, with permission, from [author names, paper title, IEEE publication title, and month/year of publication]

2) Only the accepted version of an IEEE copyrighted paper can be used when posting the paper or your thesis on-line.

3) In placing the thesis on the author's university website, please display the following message in a prominent place on the website: In reference to IEEE copyrighted material which is used with permission in this thesis, the IEEE does not endorse any of [university/educational entity's name goes here]'s products or services. Internal or personal use of this material is permitted. If interested in reprinting/republishing IEEE copyrighted material for advertising or promotional purposes or for creating new collective works for resale or redistribution, please go to http://www.ieee.org/publications standards/publications/rights/rights link.html to learn how to obtain a License from RightsLink.

If applicable, University Microfilms and/or ProQuest Library, or the Archives of Canada may supply single copies of the dissertation. 